\title{
APPLICATION OF PROBABILISTIC FRACTURE MECHANICS FOR LIFE PREDICTION OF METALLIC MATERIALS
}

\author{
A Dissertation by \\ Justin Augustinus James \\ Bachelors of Engineering, University of Malaya, 1991 \\ Masters of Science, Wichita State University, 1997
}

Submitted to the Department of Aerospace Engineering and the faculty of the Graduate School of

Wichita State University

in partial fulfillment of

the requirements for the degree of

Doctor of Philosophy

December 2007 
(C) Copyright 2007 by Justin Augustinus James

All Rights Reserved 


\section{APPLICATION OF PROBABILISTIC FRACTURE MECHANICS FOR LIFE PREDICTION OF METALLIC MATERIALS}

The following faculty have examined the final copy of this dissertation for form and content, and recommend that it be accepted in partial fulfillment of the requirement for the degree of Doctor of Philosophy with a major in Aerospace Engineering

Walter Horn, Committee Chair

Gamal Weheba, Committee Co-Chair

Roy Myose, Committee Member

Charles Yang, Committee Member

Suresh Keshavanarayana, Committee Member

Accepted for the College of Engineering

Zulma Toro-Ramos, Dean

Accepted for the Graduate School

Susan Kovar, Dean 


\section{DEDICATION}

To both my deceased parents 
There are two tragedies in life. One is not to get your heart's desire. The other is to get it. George Bernard Shaw 


\section{ACKNOWLEDGEMENTS}

I take this opportunity to thank my previous advisor Dr. James Locke who provided funding and technical support in starting this research project. I would like to thank my committee chair Dr. Horn, for his support, encouragement, and guidance in completing this study and Dr. Gamal Weheba who sowed the seed of interest to the world of probability and statistics. I take this opportunity to thank Dr. Jim Newman for his time and technical advice on this research project especially with FASTRAN-II life prediction code. I would also like to extend my gratitude to members of my committee, Drs. Roy Myose, Charles Yang, and Suresh Keshavanarayana, for their helpful comments and suggestions on all stages of this project. I also want to thank the FAA for partially funding this research project. 


\begin{abstract}
A statistically based analytical method was developed to improve the reliability of fatigue failure predictions for metallic components of various geometric configurations subjected to constant amplitude fatigue loads. The method required the determination of the distribution of equivalent initial flaw sizes. The equivalent initial flaw size associated with an actual crack is influenced by the geometric configuration of the body, the applied loading conditions and the crack growth model employed. In this study four factors were identified including two types of crack configurations and their influence on the equivalent initial flaw size distribution was investigated. Also the influence of the four factors on the equivalent initial flaw size distribution was examined. These are the stress concentration factor, $K_{\mathrm{tg}}$, the uncracked ligament length, $w_{\mathrm{n}}$, the maximum stress, $S_{\max }$ and mean stress, $S_{\text {mean }}$, of the applied constant amplitude fatigue loading. Two types of initial crack configurations were assumed; corner and surface flaw. Five specimen types were investigated; smooth unnotched, open-hole, $K_{\mathrm{t}}=3, K_{\mathrm{t}}=4$ and $K_{\mathrm{t}}=5$ specimens all of which have different stress concentration factors.

Two statistical models were fitted and investigated. One model assumed that the initial crack started as a corner crack, transitioned into a through crack, and then propagated till final fracture. The other model assumed that the initial crack started as a surface crack, transitioned into a through crack, and then propagated till final fracture. For each crack configuration, a statistical model was fitted between the mean and the standard deviation of the distributions of the equivalent initial flaw sizes and the stress concentration factor, the uncracked ligament length, the maximum stress, and the mean stress of the fatigue loading. Multiple regression techniques were used to fit these models. Using the statistical models together with Monte Carlo
\end{abstract}


simulation, a distribution of estimated fatigue lives were generated using the FASTRAN-II crack growth code for the $K_{\mathrm{t}}=3$ and $K_{\mathrm{t}}=4$ specimen types, at various stress levels. Hypothesis testing was used to compare the estimated fatigue lives of the specimen with the measured fatigue lives obtained from testing at a level of significance of 0.05 . An acceptance criterion was established to determine if the predicted fatigue lives compared well with experimentally obtained fatigue lives. As a conclusion, the methodology provided adequate approximation of fatigue life for some of the fatigue loading cases of the $K_{\mathrm{t}}=4$ specimens and for one of the fatigue loading cases of the $K_{\mathrm{t}}=3$ specimens. The initial crack configuration type did not have any significant effect on the proposed methodology. 


\section{TABLE OF CONTENTS}

Chapter

Page

1. INTRODUCTION 1

$1.1 \quad$ Research Objective ...................................................................................

1.2 Durability Characteristics ...........................................................................

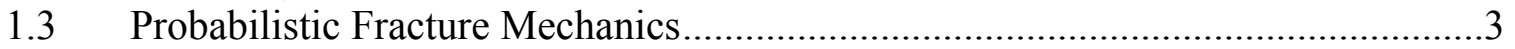

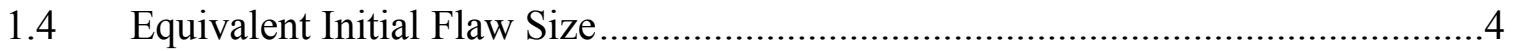

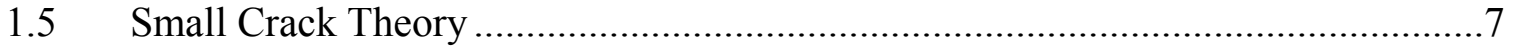

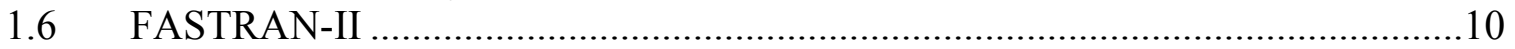

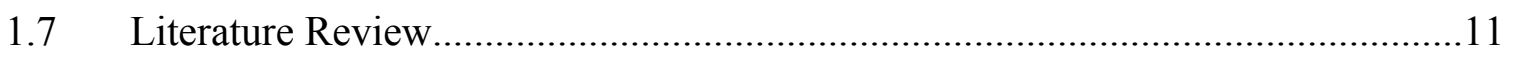

2. SCOPE OF WORK, ASSUMPTIONS, AND CHALLENGES ...................................22

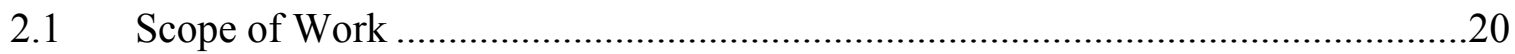

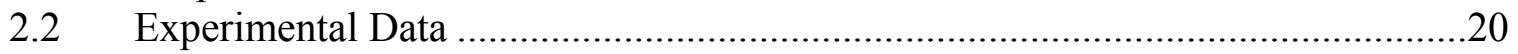

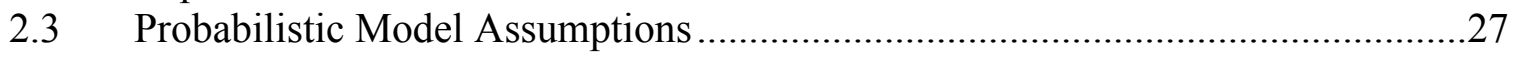

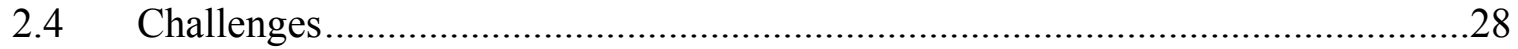

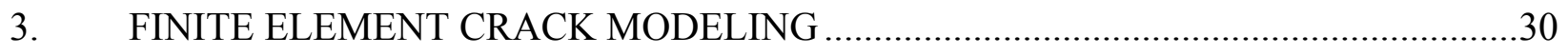

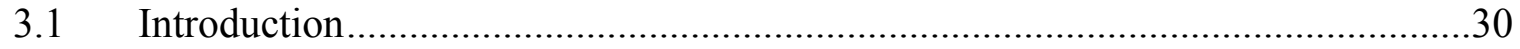

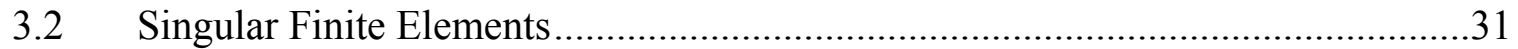

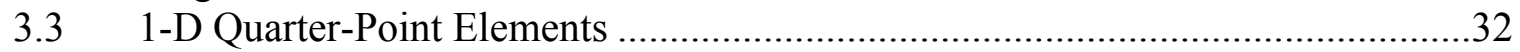

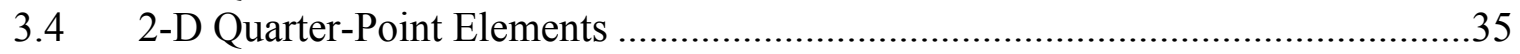

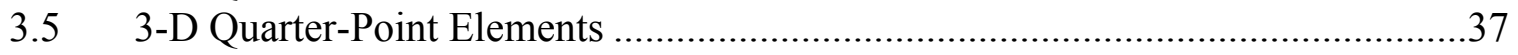

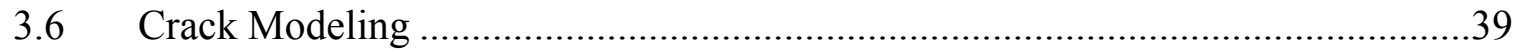

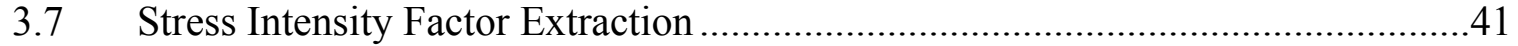

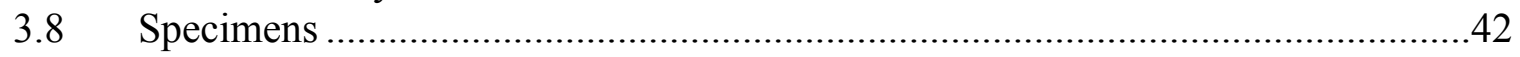

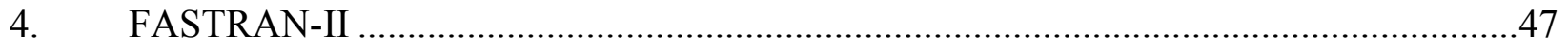

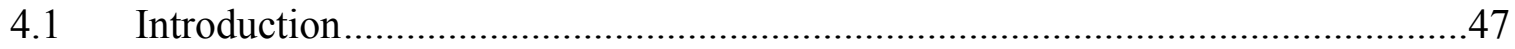

4.2 Mechanical and Fracture Properties......................................................... 47

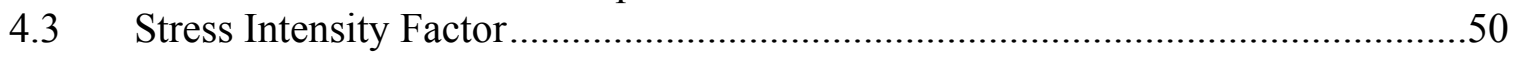

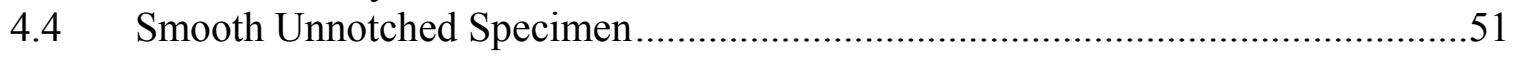

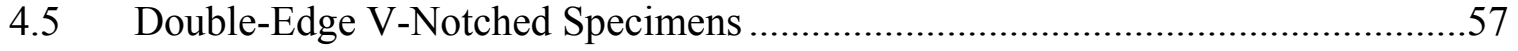

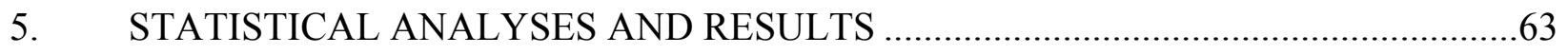

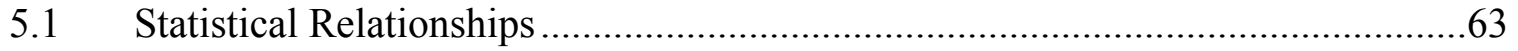

5.2 Calculating Equivalent Initial Flaw Size ..................................................64

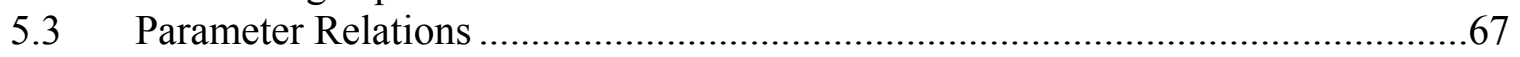




\section{TABLE OF CONTENTS (continued)}

Chapter

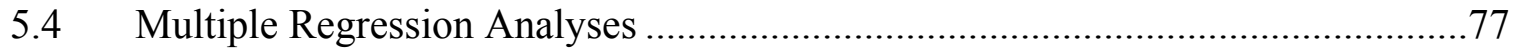

5.5 Residual Analysis of Regression Models ....................................................... 81

5.6 Hypothesis Testing of Regression Model Parameters .........................................90

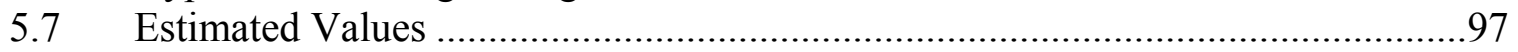

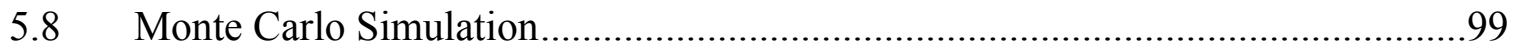

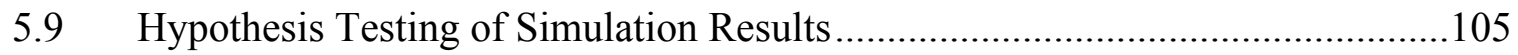

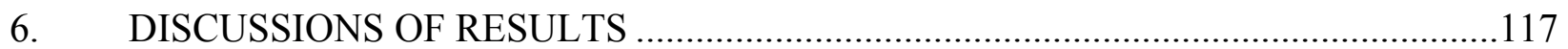

7. CONCLUSIONS AND RECOMMENDATIONS .................................................. 121

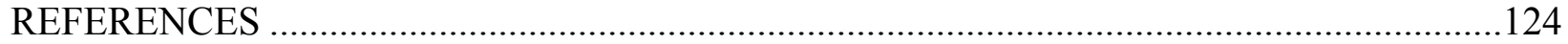

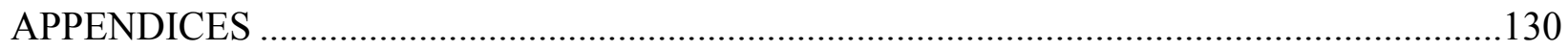

A. FASTRAN-II Input File for the Smooth Unnotched Specimen ..............................131

B. FORTRAN 90 Program to Determine the Equivalent Initial Flaw Size ..................133

C. Multiple Regression Analysis and Analysis of Variance Report ...........................137

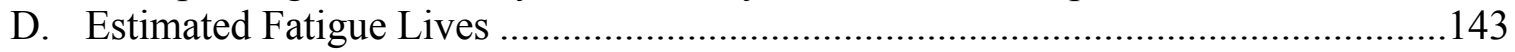




\section{LIST OF TABLES}

Table

Page

2.1 Finite element mesh details and resulting stress concentration factors for the five types of specimens of the study

4.1 Mechanical, fracture and baseline crack-growth ( $\Delta K_{\text {eff }}$-rate) properties for 2024-T3 aluminum (from Reference [1])

4.2 Limit case values for the double-edge V-notched specimens.....................................58

5.1 Key parameters multiple regression analysis and analysis of variance reports ...............97

5.2 Equivalent initial flaw size estimated mean and estimated standard deviation for $K_{\mathrm{t}}=3$ specimen $\left(K_{\mathrm{tg}}=5.165, w_{\mathrm{n}}=3.0480 \times 10^{-2} \mathrm{~m}\right)$............

5.3 Equivalent initial flaw size estimated mean and estimated standard deviation for $K_{\mathrm{t}}=4$ specimen $\left(K_{\mathrm{tg}}=7.013, w_{\mathrm{n}}=3.0480 \times 10^{-2} \mathrm{~m}\right)$

5.4 Estimated fatigue life mean and coefficient of variation for $K_{t}=3$ specimen. 104

5.5 Estimated fatigue life mean and coefficient of variation for $K_{t}=4$ specimen 105

5.6 Measured fatigue life mean and coefficient of variation for $K_{t}=3$ specimen

5.7 Measured fatigue life mean and coefficient of variation for $K_{t}=4$ specimen

5.8 $P$-values for variance hypothesis test results for $K_{\mathrm{t}}=3$ specimen .......................110

5.9 $P$-values for variance hypothesis test results for $K_{t}=4$ specimen .......................110

5.10 $P$-values for mean hypothesis test result for $K_{t}=3$ specimen.............................114

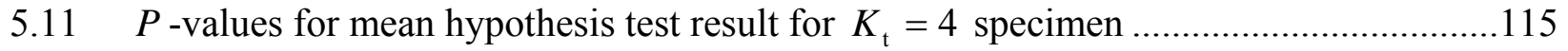

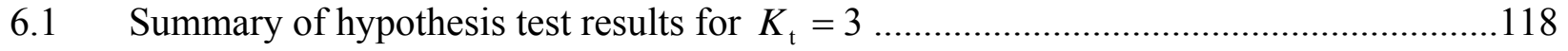

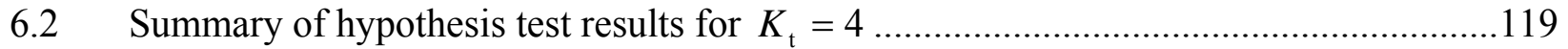

C.1(a) Multiple regression analysis report response variable: $\bar{c}_{0, \text { corner }}^{\mathrm{eq}}$ 139 


\section{LIST OF TABLES (continued)}

Table

Page

C.1(b) Analysis of variance report for response variable: $\bar{c}_{0, \text { corner }}^{\mathrm{eq}}$

C.2(a) Multiple regression analysis report response variable: $s_{\text {corner }}^{\mathrm{eq}}$

C.2(b) Analysis of variance report for response variable: $s_{\text {corner }}^{\mathrm{eq}}$

C.3(a) Multiple regression analysis report response variable: $\bar{c}_{0 \text {,surface }}^{\text {eq }}$

C.3(b) Analysis of variance report for response variable: $\bar{c}_{0, \text { surface }}^{\mathrm{eq}}$

C.4(a) Multiple regression analysis report response variable: $S_{\text {surface }}^{\mathrm{eq}}$

C.4(b) Analysis of variance report for response variable: $s_{\text {surface }}^{\mathrm{eq}}$

D.1(a) Estimated fatigue lives for $K_{\mathrm{t}}=3$ specimen and for $S_{\text {mean }}=20.684 \mathrm{~N} / \mathrm{mm}^{2}$

D.1(b) Estimated fatigue lives for $K_{\mathrm{t}}=3$ specimen and for $S_{\text {mean }}=41.369 \mathrm{~N} / \mathrm{mm}^{2}$

D.2(a) Estimated fatigue lives for $K_{\mathrm{t}}=4$ specimen and for $S_{\text {mean }}=20.684 \mathrm{~N} / \mathrm{mm}^{2}$

D.2(b) Estimated fatigue lives for $K_{\mathrm{t}}=4$ specimen and for $S_{\text {mean }}=41.368 \mathrm{~N} / \mathrm{mm}^{2}$ .150 


\section{LIST OF FIGURES}

Figure

Page

1.1 Determining equivalent initial flaw size by backward extrapolation .............................5

1.2 Typical fatigue crack growth behavior for small and large cracks ...............................

2.1 Geometric details of the smooth unnotched specimen .............................................22

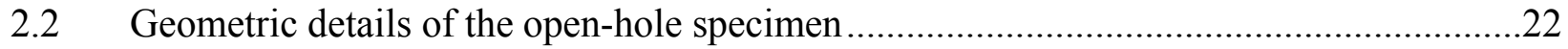

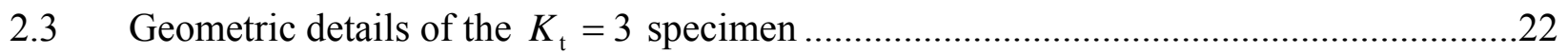

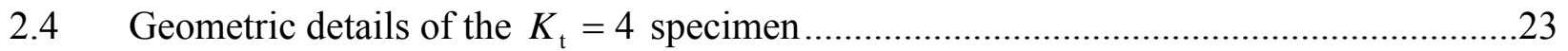

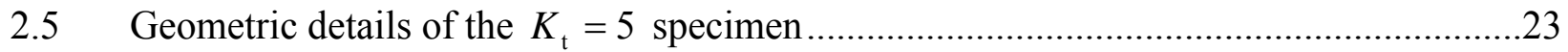

2.6 $S-N$ data from smooth unnotched specimens ................................................25

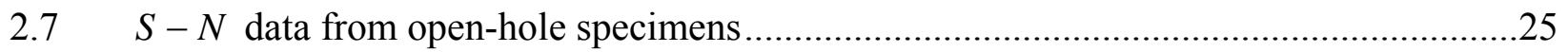

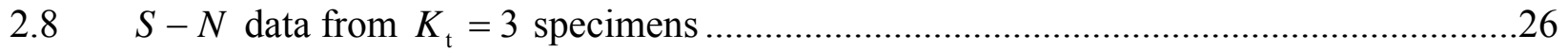

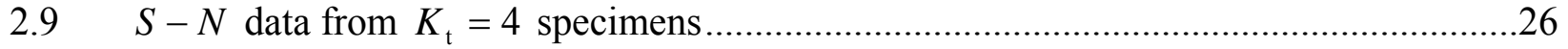

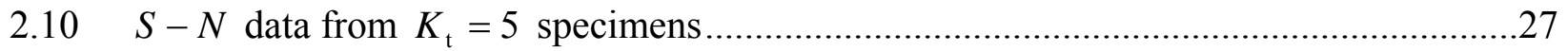

2.11 Initial corner and surface crack configuration for open-hole specimens .......................28

2.12 Initial corner and surface crack configuration for smooth unnotched specimens

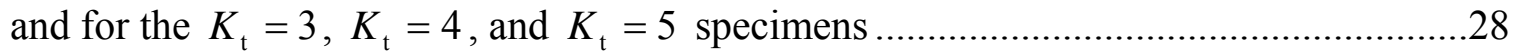

3.1 Quadrilateral and collapsed quadrilateral quarter-point elements ................................32

3.2 The 1-D quadratic element in parametric space and Cartesian space..............................33

3.3 Crack tip modeled with eight collapsed quadrilateral quadratic-point elements ..............36

3.4 Parametric space of a quadrilateral element; mapping of constant $\eta$ lines in lines in Cartesian space when node 6 is placed midpoint and eccentric midpoint between far side nodes 2 and 3 


\section{LIST OF FIGURES (continued)}

Figure

Page

3.5 The collapsed, 20-noded brick, quarter-point element .38

3.6 Flow chart of the combination of commercial codes used to determine the stress intensity factors of the specimens

3.7 Finite element grid in the vicinity of the crack tip of a smooth unnotched specimen with a corner crack $(a=c=1.016 \mathrm{~mm}$; sq112x4c crack-block; total number of elements $=1,112$; total number of nodes $=6,696$; total degrees of freedom $=20,088$ )

3.8 Finite element grid in the vicinity of the crack tip of a $K_{t}=3$ specimen with a surface crack $(a=c=0.254 \mathrm{~mm}$; two sq103x4 crack-blocks; total number of elements $=7,222$; total number of nodes $=41,066$; total degrees of freedom $=123,198)$

3.9 Finite element grid in the vicinity of the crack tip of a $K_{t}=4$ specimen with a surface crack ( $a=c=0.635 \mathrm{~mm}$; two sq112ax4 crack-blocks; total number of elements $=2,646$; total number of nodes $=15,085$; total degrees of freedom $=45,255)$

3.10 Finite element grid in the vicinity of the crack tip of a $K_{t}=5$ specimen with a through-crack ( $c=9.869 \mathrm{~mm}$; st28x1 type crack-block used; total number of elements $=2,486$; total number of nodes $=14,408$; total degrees of freedom $=43,224$ )

4.1 Crack front node set for the quarter circular corner crack .......................................51

$4.2 \quad$ Nomenclature for smooth unnotched specimen .......................................................52

4.3 Local stresses acting in the vicinity of a crack tip as the crack length, $c$, approaches zero.

4.4 $K_{\mathrm{I}}$ VS. $c$ for smooth unnotched specimen with an initial corner crack. .56

4.5 $K_{\mathrm{I}}$ vS. $c$ for smooth unnotched specimen with an initial surface crack ........................56

4.6 Nomenclature used for double-edge V-notched specimens.........................................57

$4.7 \quad K_{I}$ VS. $c$ for $K_{t}=3$ specimen with an initial corner crack ..................................60 


\section{LIST OF FIGURES (continued)}

Figure

Page

4.8 $K_{\mathrm{I}}$ vs. $c$ for $K_{\mathrm{t}}=3$ specimen with an initial surface crack .60

4.9 $K_{\mathrm{I}}$ vs. $c$ for $K_{\mathrm{t}}=4$ specimen with an initial corner crack.

4.10 $K_{\mathrm{I}}$ vs. $c$ for $K_{\mathrm{t}}=4$ specimen with an initial surface crack.

4.11 $K_{\mathrm{I}}$ vs. $c$ for $K_{\mathrm{t}}=5$ specimen with an initial corner crack

4.12 $K_{\mathrm{I}}$ vs. $c$ for $K_{\mathrm{t}}=5$ specimen with an initial surface crack

5.1 Flow chart depicting the iterative procedure involved in the calculation of equivalent initial flaw size.

5.2 The relationship of $c_{0}$ as a function of the $S_{\max }$ for the smooth unnotched specimens with a $S_{\text {mean }}$ of $144.790 \mathrm{~N} / \mathrm{mm}^{2}$

5.3 The relationship of $c_{0}$ as a function of the $S_{\max }$ for the open-hole specimens with a $S_{\text {mean }}$ of $20.684 \mathrm{~N} / \mathrm{mm}^{2}$

5.4 The relationship of $c_{0}$ as a function of the $S_{\max }$ for the open-hole specimens with a $S_{\text {mean }}$ of $41.369 \mathrm{~N} / \mathrm{mm}^{2}$

5.5 The relationship of $c_{0}$ as a function of the $S_{\max }$ for the $K_{\mathrm{t}}=5$ specimens with a $S_{\text {mean }}$, of $20.684 \mathrm{~N} / \mathrm{mm}^{2}$

5.6 The relationship of $c_{0}$ as a function of the $S_{\max }$ for the $K_{\mathrm{t}}=5$ specimens with a $S_{\text {mean }}$, of $41.369 \mathrm{~N} / \mathrm{mm}^{2}$

5.7 The relationship between $\bar{C}_{0}$ and $S_{\max }$ for smooth unnotched, open-hole and $K_{\mathrm{t}}=5$ specimens

5.8 The relationship between $s$ and $S_{\max }$ for smooth unnotched, open-hole and $K_{\mathrm{t}}=5$ specimens 


\section{LIST OF FIGURES (continued)}

Figure

Page

5.9 The relationship between $\bar{C}_{0}^{\mathrm{eq}}$ and $S_{\max }$ for smooth unnotched, open-hole and $K_{t}=5$ specimens .76

5.10 The relationship between $S^{\mathrm{eq}}$ and $S_{\max }$ for smooth unnotched, open-hole and $K_{\mathrm{t}}=5$ specimens .76

5.11 Residual normal probability plot for the $\hat{\bar{C}}_{0, \text { corner }}^{\text {eq }}$ model .86

5.12 Residual normal probability plot for the $\hat{\bar{C}}_{0, \text { surface }}^{\text {eq }}$ model .86

5.13 Residual normal probability plot for the $\hat{s}_{\text {corner }}^{\text {eq }}$ model .86

5.14 Residual normal probability plot for the $\hat{s}_{\text {surface }}^{\text {eq }}$ model .86

5.15 Studentized residual plot for estimated equivalent mean .89

5.16 Studentized residual plot for estimated equivalent standard deviation

$5.17 C_{0}$ normal probability plot for the smooth unnotched specimen $\left(S_{\text {mean }}\right.$ of $144.684 \mathrm{MPa}$ and $S_{\max }$ of $210.290 \mathrm{MPa}$ )

$5.18 C_{0}$ normal probability plot for the open-hole specimen $\left(S_{\text {mean }}\right.$ of 20.684 MPa and $S_{\max }$ of $124.106 \mathrm{MPa}$ )

$5.19 C_{0}$ normal probability plot for the open-hole specimen $\left(S_{\text {mean }}\right.$ of $41.369 \mathrm{MPa}$ and $S_{\max }$ of $79.290 \mathrm{MPa}$ )

$5.20 C_{0}$ normal probability plot for the $K_{\mathrm{t}}=5$ specimen $\left(S_{\text {mean }}\right.$ of $20.684 \mathrm{MPa}$ and $S_{\max }$ of $32.750 \mathrm{MPa}$ )

$5.21 C_{0}$ normal probability plot for the $K_{\mathrm{t}}=5$ specimen $\left(S_{\text {mean }}\right.$ of $41.369 \mathrm{MPa}$ and $S_{\max }$ of $62.053 \mathrm{MPa}$ ) 


\section{LIST OF SYMBOLS}

$a$

C

$F(c)$

$K_{\mathrm{F}}$

E

$m$

$R$

$r$

$S_{\text {app }}$

$\sigma_{g}$

$S_{\text {max }}$

$S_{\text {min }}$

$S_{\text {mean }}$

$\alpha_{\text {constraint }}$

$\Delta K$

$\Delta K_{\text {eff }}$

$\Delta K_{\text {th }}$

$\frac{d a}{d N}, \frac{d c}{d N}$

$\sigma_{\mathrm{ys}}$
Crack depth in thickness direction, $m$ or $m m$

Crack length in width direction, $m$ or $\mathrm{mm}$

Boundary-correction factor

Elastic-plastic fracture toughness in Two-Parameter Fracture

Criterion, $M P a \sqrt{m}$

Young's modulus of elasticity, $M P a$

Fracture toughness parameter in Two-Parameter Fracture Criterion

Stress ratio

Notch or hole radius, $m$ or $m m$

Applied stress, $M P a$

Remote gross stress, $M P a$

Maximum applied stress, $M P a$

Minimum applied stress, $M P a$

Mean applied stress, $\mathrm{MPa}$

Constraint factor

Stress-intensity factor range, $M P a \sqrt{m}$

Effective stress-intensity factor range, $M P a \sqrt{m}$

Large crack $\Delta K$ threshold, $M P a \sqrt{m}$

Crack growth rate, $m /$ cycles

Yield stress ( 0.2 percent offset), $M P a$ 


\section{LIST OF SYMBOLS (continued)}

$\sigma_{\mathrm{u}}$

$K_{\text {tg }}$

$w_{\mathrm{n}}$

w

$K_{\text {I }}$

$\sigma_{y y}$

$c_{\mathrm{U}}$

$c_{\mathrm{L}}$

$C_{\text {approx }}$

$N_{\text {FAST }}\left(c_{\mathrm{U}}\right)$

$N_{\text {FAST }}\left(c_{\mathrm{L}}\right)$

$C_{0, \text { corner or surface }}$

$\bar{C}_{0, \text { corner or surface }}$

$S_{\text {corner or surface }}$

$\bar{C}_{0, \text { corner or surface }}^{\text {eq }}$

$S_{\text {corner or surface }}^{\mathrm{eq}}$

$\hat{\bar{C}}_{0, \text { corner or surface }}$

$\hat{S}_{\text {corner or surface }}$

$\beta_{i}$
Ultimate tensile strength, $M P a$

Stress concentration factor

Uncracked ligament length, $m$ or $\mathrm{mm}$

Width, $m$ or $m m$

Mode I stress intensity factor

Local stress, $M P a$

Initial flaw size-FASTRAN-II input

Initial flaw size-FASTRAN-II input

Approximated initial flaw size

FASTRAN-II predicted live

FASTRAN-II predicted live

Equivalent initial flaw size

Equivalent initial flaw size mean at a specific $S_{\max }$

Equivalent initial flaw size standard deviation at a specific $S_{\max }$

Equivalent initial flaw size equivalent mean at a specific $S_{\max }$

Equivalent initial flaw size equivalent standard deviation at a specific $S_{\max }$

Equivalent initial flaw size estimated mean at a specific $S_{\max }$

Equivalent initial flaw size estimated standard deviation at a specific $S_{\max }$

Regression coefficient 


\section{LIST OF SYMBOLS (continued)}

$\hat{\beta}_{i}$

$\varepsilon$

$\hat{\bar{C}}_{0, \text { corner or surface }}^{\text {eq }}$

$\hat{S}_{\text {corner or surface }}^{\mathrm{eq}}$

$S S_{\text {total }}$

$S S_{\text {regression }}$

$S S_{\text {error }}$

$M_{S S}$ total

$M S S_{\text {regression }}$

$M_{S S}$ error

$e$

$e_{\text {studentized }}$

$E(e)$

Z

$\mathrm{H}_{0}$

$\mathrm{H}_{\mathrm{a}}$

F

$t$

$\alpha$

$P$ - value
Estimated regression coefficient

Random error

Equivalent initial flaw size estimated equivalent mean

Equivalent initial flaw size estimated equivalent standard deviation

Total sum of squares

Regression sum of squares

Error sum of squares

Mean total sum of squares

Mean regression sum of squares

Mean error sum of squares

Residual

Studentized residual

Expected residual

Random variable (standard normal distribution)

Null hypothesis

Alternative hypothesis

Random variable ( $F$-distribution)

Random variable ( $t$-distribution)

Level of significance

Observed level of significance 


\section{LIST OF SYMBOLS (continued)}

$\begin{array}{ll}R^{2} & \text { Multiple coefficient of determination } \\ R_{\text {adj }}^{2} & \text { Adjusted multiple coefficient of determination } \\ n & \text { Sample size of measured fatigue life } \\ N & \text { Measured fatigue life } \\ \bar{N} & \text { Measured fatigue life mean } \\ s_{\bar{N}} & \text { Measured fatigue life standard deviation } \\ c_{v} & \text { Measured fatigue life coefficient of variation } \\ \mu & \text { Measured fatigue life population mean } \\ \sigma^{2} & \text { Measured fatigue life population variance } \\ n^{\mathrm{MC}} & \text { Sample size of estimated fatigue life } \\ N_{\text {corner or surface }}^{\mathrm{MC}} & \text { Estimated fatigue life } \\ \bar{N}_{\text {corner or surface }}^{\mathrm{MC}} & \text { Estimated fatigue life mean } \\ s_{\bar{N}, \text { corner or surface }}^{\mathrm{MC}} & \text { Estimated fatigue life standard deviation } \\ c_{v, \text { corner or surface }}^{\mathrm{MC}} & \text { Estimated fatigue life coefficient of variation } \\ \mu_{\mathrm{corner}}^{\mathrm{MC} \text { o surface }} & \text { Estimated fatigue life population mean } \\ \left(\sigma_{\text {corner or surface }}^{\mathrm{MC}}\right. & \text { Estimated fatigue life population variance }\end{array}$




\section{CHAPTER 1}

\section{INTRODUCTION}

\subsection{Research Objective}

The main objective of this study was to develop a statistical based analytical method for generating reliable fatigue failure estimations for metallic components of various geometric configurations subjected to constant amplitude stress levels. The method requires a minimum amount of experimental data and accounts for stress concentration effects. This methodology could greatly reduce the number of required fatigue tests and thus the cost associated with fatigue testing.

Fatigue failure is essentially a random phenomenon. Customary methods of deterministic analyses often produce inconclusive, or conflicting, estimates of the fatigue life of a component. In order to overcome this drawback, a methodology based on a deterministic fracture mechanics crack growth approach was augmented with a statistical approach to predict the life of the component.

This durability analysis, based on a probabilistic fracture mechanics approach, takes into account the initial fatigue quality (i.e., surface treatment, manufacturing defects), fatigue-crack growth, load spectra, and structural design details. An equivalent initial flaw size distribution was used to characterize the initial fatigue quality of the component. By treating the equivalent initial flaw size as a random continuous variable, an equivalent initial flaw size distribution was obtained by fitting a statistical distribution to a set of equivalent initial flaw size data. In practice the initial flaw value is less $0.1 \mathrm{~mm}$ and is considered to be a small crack (Reference [1]), therefore small crack-effects must be accounted for in the evaluation of the equivalent initial flaw value. In order to satisfy this condition FASTRAN-II, a life prediction software developed 
by Newman [2] based on small crack theory, together with measured fatigue life data from a recent investigation by Locke [3] was used to calculate the equivalent initial flaw size associated with each measured failure point.

The equivalent initial flaw size associated with the measured failure point is influenced by the geometric configuration, fatigue loading conditions and the crack growth model employed. In this study four factors were identified and their influence on the equivalent initial flaw size distribution was investigated. These four factors included two factors associated with the geometric configuration-stress concentration factor, $K_{\mathrm{tg}}$, and uncracked ligament length, $w_{\mathrm{n}}$, and two factors associated with the constant amplitude cyclic loading-the maximum stress, $S_{\max }$, and the mean stress, $S_{\text {mean }}$ of the fatigue load.

A statistical model was established to determine the relation between equivalent initial flaw size distribution and these four factors. Multiple regression analyses were performed to calibrate this statistical model. This model was able to provide equivalent initial flaw size distribution parameters for a given $S_{\text {max }}, S_{\text {mean }} K_{\text {tg }}$, and $w_{\mathrm{n}}$. Once the statistical model was calibrated an equivalent initial flaw size distribution could be generated for given values of these four parameters and used as input in the FASTRAN-II code to generate component fatigue life distribution using a Monte Carlo simulation. The model was benchmarked against data obtained from experiments.

A major portion of this study was funded through the Data and Methodologies for Structural Life Evaluation of Small Airplanes project conducted at Wichita State University from 2004 to 2006 [3]. 


\subsection{Durability Characteristics}

The durability of structural components subjected to high-cycle fatigue environments can be characterized by an $S-N$ curve, a graph of the number of cycles of fatigue load, $N$, at a specific magnitude of stress, $S$, required to fail the material.

Material $S-N$ curves are derived from coupon tests of the subject material. A regular sinusoidal stress of magnitude $S$ is applied and the number of cycles to failure is measured. Each coupon test generates a single point on the curve. For sufficiently small stress levels there is a runout where the required cycles to failure would require more time than that available for the test.

Test specimens used in fatigue tests are polished to minimize the effects of surface imperfections. Imperfections such as inclusions, small scratches and dents can generate stress concentrations that produce crack initiation sites and reduce the endurance limit. Statistical techniques are usually employed to fit an analytical curve to the measured data in order to generate the final $S-N$ curve.

\subsection{Probabilistic Fracture Mechanics}

In engineering applications, customary methods of deterministic analysis often produce inconclusive, unrealistic predictions of component reliability. In order to account for this shortcoming, probabilistic fracture mechanics is often employed. The application of probabilistic fracture mechanics in its pure form presents some limitations, but not as severe as those associated with a pure deterministic analysis. Generally the methods of probabilistic fracture mechanics are based upon deterministic fracture mechanics procedures. A probabilistic fracture mechanics model is calibrated against all relevant experience. That is, unknown model 
parameters are determined by statistical means from a database of credible in-service observations and reliable experimental data. The calibrated probabilistic fracture mechanics model can provide accurate reliability predictions for a specific structural component.

\subsection{Equivalent Initial Flaw Size}

In practice it has been found that preexisting manufacturing defects or service induced damage (scratches, flaws, burrs, cracks, and corrosion pits) are very often the initial source of structural cracking for components subjected to cyclic loads. The impact of these defects on the life of a component is dependent on the defect's initial size, the rate of crack growth with service usage, and the critical crack size.

From these considerations, the "damage-tolerance" design philosophy was developed (Gallagher et al. [4]). The damage-tolerance approach assumes that a component has prior damage, or a flaw of a size that is inspectable. The useful service life of the component can then be determined to be a specified portion of the crack propagation life required to grow the crack from this inspectable size to a crack length at the onset of unstable crack growth.

The durability design concept refers to a procedure to determine an economic-life extension that minimizes in-service maintenance costs and maximizes performance of the component (Manning and Yang [5]). The durability analysis methodology based on a probabilistic fracture mechanics approach, accounts for the initial fatigue quality (i.e., surface treatment, manufacturing defects), fatigue-crack growth, load spectra, and structural design details.

An equivalent initial flaw size distribution is used to characterize the initial fatigue quality. This equivalent initial flaw size distribution is obtained by fitting a statistical 
distribution to equivalent initial flaw size data sets. An equivalent initial flaw is a hypothetical crack assumed to exist in the structure prior to service. There is no direct physical relationship between equivalent initial flaw size cracks and actual initial flaws in the structure. The equivalent initial flaw size is an analytical creation that can be determined by using a simple crack growth model to grow a crack of inspective size, usually about $0.762 \mathrm{~mm}$, backwards to a time equal to zero. This is illustrated in Figure 1.1 below.

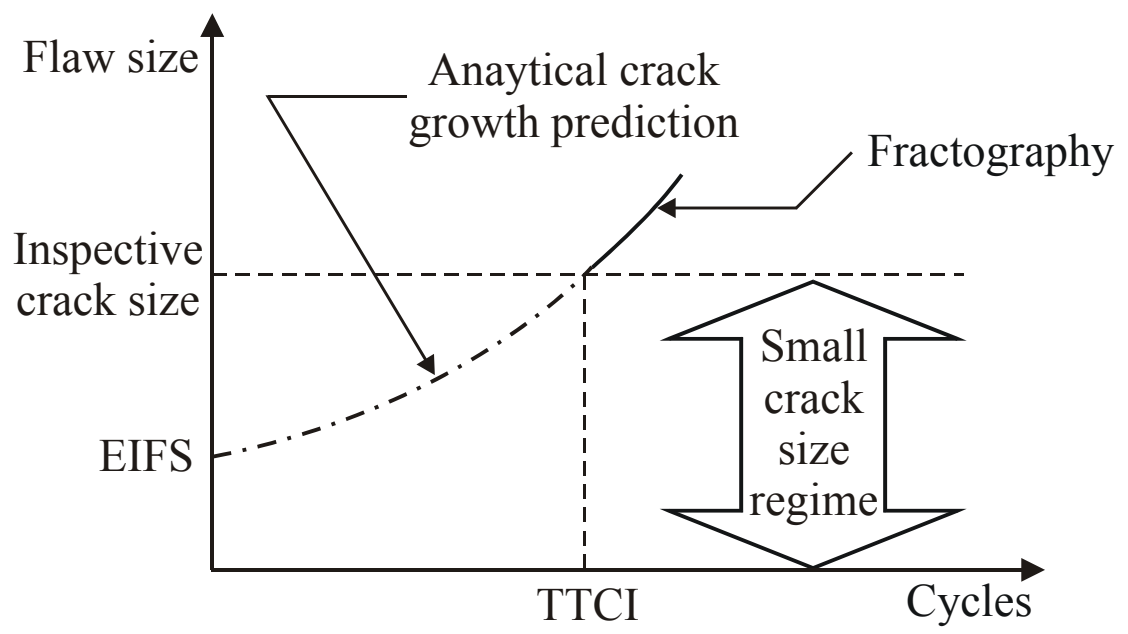

Figure 1.1 Determining equivalent initial flaw size by backward extrapolation (Courtesy of Yang and Manning [6])

Figure 1.1 depicts a typical plot of flaw size (crack length) as a function of the number of cycles of loading for a specimen subjected to constant amplitude cyclic loading or to the more general spectrum loading. The number of cycles taken to initiate a crack of inspective size (e.g., $0.762 \mathrm{~mm}$ ) is called the time-to-crack-initiation (TTCI). An equivalent initial flaw size (EIFS) can be determined by extrapolating backwards in time a crack of inspective size at time-to-crackinitiation to a time equal to zero using an analytical crack growth prediction model as illustrated 
by dash dot curve in Figure 1.1. Equivalent initial flaw sizes are usually less than $0.1 \mathrm{~mm}$ and therefore fall in the small crack size regime.

Equivalent initial flaw size is not a material property. That is, it is not unique for a specific material, but rather it can be affected by many factors such as the material type, manufacturing quality, stress gradient, crack configuration, residual stress, level of stress, load type and severity, crack growth model, and small crack behavior.

As the equivalent initial flaw size becomes smaller (less than $0.1 \mathrm{~mm}$ ), the question of small crack effect must be addressed. A detailed explanation of the small crack effect will be presented in Section 1.5. Equivalent initial flaw size variability with load spectra may be resolved by implementing small crack theory in the analysis.

The evolution of both the "safe life" and the "durability/damage tolerance" design concepts have moved in the direction of "small crack behavior". As manufacturing quality improves, smaller initial flaw sizes are produced in manufacturing and likewise, as inspection techniques improve with advances in technology smaller initial flaws can be detected. The design of uninspectable (or geometrically hidden) structures, or structures subjected to an extremely large number of cyclic loads (i.e. engines and helicopter components) must rely on approaches that deal with the growth of small cracks.

Small crack theory, as opposed to large crack theory, can be used to assess the structural fatigue life of a component at two levels of initial flaw sizes. First, it can be used to assess the initial design quality of a structure based on the material microstructural properties, such as cracks growing from inclusion particles, voids, grain boundaries, or cladding layers. In the second level of calculations, as in the durability analysis, a manufacturing flaw or serviceinduced flaw is used along with small crack theory to determine the remaining life of the 
structural component. Thus, the analysis can account for the effects of small crack growth, which has a faster growth rate at a given linear elastic stress intensity factor range, $\Delta K$ than the large crack theory would predict. Furthermore, crack growth is possible for $\Delta K$ below the threshold of large crack growth. Thus small crack theory can be used to determine the equivalent initial flaw size for various load spectra and structural details, and to characterize the $S-N$ behavior of the material.

\subsection{Small Crack Theory}

Most fatigue life variations appear to originate, either directly or indirectly, from microstructural inhomogeneities, such as inclusion particles, voids, slip-bands or from manufacturing defects but the exact mechanisms responsible for the variability remain to be

identified. Traditionally, the total fatigue life of a mechanical component has been divided into the following three phases: crack nucleation, small crack growth, and finally large crack growth to fast fracture (Schijve [7]). Previous investigators of small crack growth [8-10] have observed that fatigue failures of many engineering materials were initiated primarily by crack growth from microstructural features. Thus, "small crack theory" can be considered to be the treatment of the response to fatigue loading as a crack propagation process from micro-defect to failure. The life due to crack nucleation is generally assumed to be negligible. The initial size of the microstructural feature that is the origin of the fully developed crack is termed the initial flaw size.

In structural component design, the application of fracture mechanics concepts to characterize large crack growth and fracture is well founded. However, in the past decade, a great deal of emphasis has been placed on extrapolating fracture mechanics theory to account for 
very small cracks of the order of $10 \mu m$ to $1 \mathrm{~mm}$. A number of linear elastic fracture mechanics studies (Pearson [11]; Ritchie and Lankford [12]; Miller and de los Rios [13]) on small cracks have revealed that small cracks tend to grow much faster than would be predicted from large crack theory and measured data and, they grow at a $\Delta K$ well below the large crack threshold stress intensity factor range, $\Delta K_{\text {th }}$. Studies by Zocher [14], Newman and Edwards [8] and in addition, Edwards and Newman [15] concluded that small crack behavior is more prevalent with negative stress ratios.

Very early in small crack research, fatigue crack closure was recognized as a possible explanation for the rapid small crack growth rates (Elber [16] and Nisitani and Takao [17]). It was hypothesized that fatigue crack closure was caused by the residual plastic deformation left in the wake of an advancing crack. Elber [16] introduced the effective stress intensity factor range, $\Delta K_{\text {eff }}$ in his linear elastic fracture mechanics analysis as the portion of the load cycle for which the crack is fully open. A steady-state crack is one in which the residual plastic deformations and crack closure along the crack surfaces are fully developed and stabilized under steady-state loading. If a small crack were to be fully open, then the $\Delta K$ would be fully effective and the crack-growth rate would be greater than the steady-state crack-growth rate. Small crack growth rates are also faster than steady-state behavior because these cracks may initiate and grow in weak microstructure environments.

Figure 1.2 below illustrates the small crack behavior relative to the long crack behavior. In this figure the fitted curves associated with the measured crack growth rate, $d a / d N$ or $d c / d N$ data for both small cracks and large cracks has been plotted against the corresponding linear elastic stress intensity factor range, $\Delta K$. 


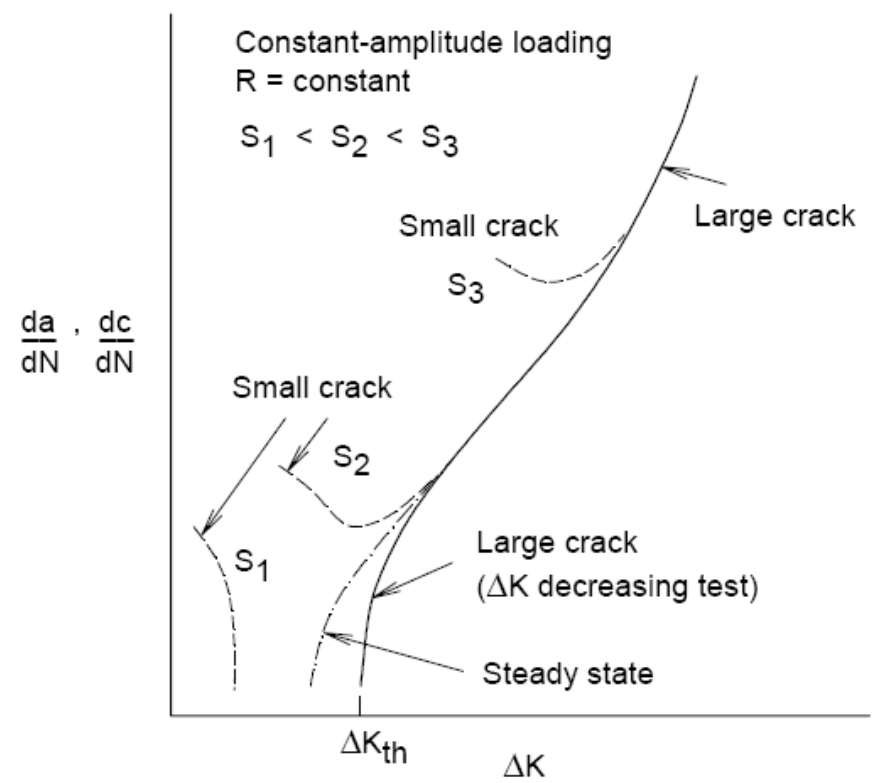

Figure 1.2 Typical fatigue crack growth behavior for small and large cracks (Courtesy of Newman et al. [1])

The solid sigmoidal curve is representative of data obtained from tests with large cracks for a given material and environment under constant amplitude loading. At low growth rates, the threshold stress intensity factor range, $\Delta K_{\text {th }}$, is usually obtained from load reduction ( $\Delta K$ decreasing) tests. The development of the large-crack threshold has also been associated with a rise in crack-opening load as the applied load is reduced (Minikawa and McEvily [18]; Newman [19]). Some typical experimental results for small cracks in plates and at notches are illustrated as the dashed curves. These results indicate that small cracks grow at $\Delta K$ levels below the large crack threshold and that they also can grow faster than large cracks at the same $\Delta K$ level above the threshold. The large crack threshold is associated with an increase in crack closure, thus the steady state crack growth behavior for the large crack is given by the dash-dot curve of the figure. On the other hand, small crack behavior has long been associated with a lack of closure 
in the early stages of growth (small cracks) consequently the greater growth rates of the dashed curves of the figure.

\subsection{FASTRAN-II}

FASTRAN-II is a fatigue life prediction computer code based on the plasticity-induced crack-closure model developed by Newman [20]. The program calculates crack length as a function of cycles from a user specified initial crack size for many common crack configurations found in structural components. The crack-closure model can be used to calculate the stress level at which a fatigue crack tip becomes fully open during cyclic loading. The applied cyclic loads can be of the form of constant-amplitude, variable-amplitude, or spectrum loading. Either tensile or compressive loads can be applied to a variety of crack configurations. The code used for the analyses of this study contained seventeen predefined crack configurations and the option for the user to define one other crack configuration by programming the stress-intensity factor equation in a user defined subroutine. The crack-opening stresses were calculated as a function of load history and crack length using either contact stress-intensity factors or crack-surface displacement equations; and, the $\Delta K_{\text {eff }}$ was either elastic or modified for plastic yielding at the crack tip (Newman [21]). Many of the input and internal variables used in the computer program were defined in Newman [2].

The crack-closure model was developed for a central crack in a finite-width specimen subjected to a uniform applied stress (Newman [20]). The model was later extended to through cracks emanating from a circular hole in a finite-width specimen subjected to a uniform applied stress (Newman [22]). The crack closure model was based on the Dugdale plastic zone model [23] that was modified to consider the plastically deformed material in the wake of the crack. 
The primary advantage in using this model was that the plastic zone size and crack-surface displacements were obtained by the superposition of two elastic problems - a crack in a plate subjected to a remote uniform stress and a crack in a plate subjected to a uniform stress acting over a segment of the crack surface.

This model also accounts for the 3-D constraint effects (Newman et al. [24]) related to the buildup of stresses around a crack front due to the restraint against in-plane and out-of-plane deformation. The degree of out-of-plane constraint is primarily influenced by the plate thickness, whereas the in-plane constraint has been associated with the distance of the crack front to external boundaries. Constraint effects play a strong role in the fatigue initiation and crack growth process.

The FASTRAN-II code had several additional options not available in the original version of FASTRAN. The revised program had the ability to model different crack growth properties in the crack depth, $a$ and crack length, $c$ directions; to model surface or corner cracks; and to accommodate properties given in either equation or tabular form. FASTRAN-II also provided the capability to input user specified spectrum loadings. Also, several additional flight-load spectra were added to the preexisting standard load spectra.

\subsection{Literature Review}

Yang and Manning [6] used the concept of the equivalent initial flaw size to characterize the initial fatigue quality of metallic structures. In their study of open-hole specimens of 7475T7351 aluminum alloy with $15 \%$ load transfer when subjected to the load spectrum of a $\mathrm{F}-16$ fighter with a maximum gross stress of 234.4 $\mathrm{MPa}$, fatigue data were collected and used in regression analyses to calibrate a simple crack growth rate equation of the form, 


$$
\frac{d a(t)}{d t}=Q a^{b}(t)
$$

Yang and Manning concluded that the general notion of an equivalent initial flaw size distribution was a useful concept for durability analyses of structures and for quantifying the economic life (e.g., probability of crack exceedance) of a structural component at the design level, or in service. As a result, the equivalent initial flaw size distribution would have a wide range of applications, including risk assessment of aging aircraft, durability analysis, multisite damage prediction, and scheduling for service, inspection, and maintenance.

Yang et al. [25] randomized equation (1.1) by introducing a lognormal random variable to account for crack growth rate dispersion. This probabilistic model together with the equivalent initial flaw size concept was used to evaluate the durability of relatively small cracks $(<2.54 \mathrm{~mm})$ in fastener holes. This model accounted for crack growth rate dispersion. Fatigue crack growth test results for 7475-T7351 aluminum specimens with filled hole containing an unloaded protruding head steel bolt with a clearance fit subjected to fighter and bomber load spectra were used to evaluate the initial fatigue quality model, and the model calibration procedures. A formulation to predict the cumulative distribution of crack size at any given time and the cumulative distribution of the time-to-crack initiation at any given crack size was developed using the derived equivalent initial flaw size distribution together with the stochastic crack growth approach. A comparison of the predictions using the formulation to actual test data in the small crack region indicated reasonable agreement. The authors also concluded that either a stochastic or a deterministic approach was satisfactory for durability analyses of aluminum alloys in the small crack region. However, the deterministic approach was less cumbersome mathematically. 
Manning et al. [26] applied the equivalent initial flaw size concept in the durability analyses of the lower wing skins of a fighter aircraft and test specimens with complex splices subjected to a bomber load spectrum. In the first part of their study both right and left wing skins were subjected to the equivalent of 16,000 flight hours using a repeatable block spectrum. Fractographic evaluations identified a total of 33 fastener holes had cracks with lengths greater than, or equal to, $0.76 \mathrm{~mm}$. The durability analysis of each wing skin was divided into ten regions based on stress levels, and the initial fatigue quality model parameters were calibrated from data sets obtained in the investigation reported by Speaker et al. [27]. The authors reported that the extent of predicted damage results tracked very well with the average test results for each individual stress region. Approximately $1.1 \%$ of the fastener holes in each fighter lower wing skin were predicted to have a crack size $\geq 0.76 \mathrm{~mm}$, while the test average for both the right and left hand lower wing skins was $1.02 \%$.

The second part of their study included durability analyses of complex-splice specimens subjected to a bomber load spectrum. Eleven specimens made of 7475-T7351 aluminum plate fastened with countersunk steel rivets were tested with cyclic loads that simulated two service lifetimes (27,000 flight hours) or failure, whichever came first. From fractographic evaluations of the test specimens, 25 out of the 110 fastener holes in the outer rows had a crack size $\geq 1.27$ $\mathrm{mm}$ after 13,500 hours had been simulated.

The initial fatigue quality model parameters of the fastener holes were calibrated based on the fractographic results for nine data sets and three different reference crack sizes. Each specimen was made of 7475-T7351 aluminum and contained two countersunk rivets. Load transfer levels of $15 \%, 30 \%$ and $40 \%$ were considered during the study. 
After 13,500 hours, the model predicted that nine fastener holes would exceed a crack size of $1.27 \mathrm{~mm}$ as compared to the fractographic test results of 25 . The difference in the predicted crack exceedances and the measured exceedances were attributed mainly to the stress level used in the predictions. The actual stress level and distribution in the outer row of fastener holes was far more complex, than those considered in the crack exceedance predictions due to lateral bending effects. The crack exceedance predictions were very sensitive to the gross applied stress level used.

In their conclusions, the authors commented on the need to refine existing methods in order to optimize the estimation of the equivalent initial flaw size distribution parameters using pooled fractographic results, and to determine the sensitivity of these parameters to material, geometry, crack size range, stress levels, amount of bolt load transfer and load spectra. They also recommended the investigation of the accuracy and limits of the durability analysis in order to increase confidence in the predictions for a broad range of applications.

Using the wing skin data reported in [26], Yang and Manning [28] performed durability analyses based on equivalent initial flaw size distributions using two different approaches. Each approach was divided into two segments; small crack size lengths less than $1.27 \mathrm{~mm}$ and large crack size lengths greater than $1.27 \mathrm{~mm}$. In the first approach both segments were treated in a deterministic manner, while in the second approach the small and large crack size regions were treated in a deterministic manner as well as a stochastic manner (Yang et al. [25]). The crack growth rate expression of Equation (1.1) was used in both approaches.

Analytical predictions for the extent of damage (i.e., number of fastener holes with cracks exceeding specified sizes), based on both approaches were compared with experimental results. 
In both approaches good correlations were obtained. The first approach was found to be more conservative than the second.

Yang et al. [29] introduced a statistical based analytical function that was capable of providing an equivalent initial flaw size distribution for different geometry configurations. This function was based upon the Weibull weakest link hypothesis and included relevant geometric parameters and the effective surface area of the critical location in its derivation. A baseline equivalent initial flaw size distribution was established for smooth unnotched specimens with a surface flaw using the $S-N$ data for smooth unnotched specimens and a least square fit. By determining the relevant geometric parameters for open-hole and double-edge notched specimens, theoretical equivalent initial flaw size distribution functions were established for each individual specimen type. Experimentally based equivalent initial flaw sizes were determined using the Paris crack growth law for both long and short crack assumption. All experimental data used in this study were for 7050-T7451 aluminum alloy, subjected to constant amplitude loading, with a stress ratio $(R)$ of 0.1 , and various stress levels. The authors concluded that the predicted equivalent initial flaw size cumulative distributions correlated well with the experimentally based results.

Yang et al. [30] applied the analytical methodology employed in [29] for determining the equivalent initial flaw size distribution for 2024-T3 aluminum alloy specimens with limited fatigue data. Experimentally based equivalent initial flaw sizes were determined using FASTRAN-II for both short and long crack behavior with elastic-plastic effects. The investigation was conducted using constant amplitude fatigue results $(R=0)$ for electropolished smooth unnotched and open-hole 2024-T3 aluminum specimens. A wide range of stress levels was considered, including stress levels above yield and stresses near the endurance limit. A 
baseline equivalent initial flaw size distribution was established from smooth unnotched specimens. By taking into account effective surface area and flaw type, theoretical equivalent initial flaw size distribution functions were derived for two sets of open-hole specimens. The theoretical and experimentally derived equivalent initial flaw distributions computed with FASTRAN-II compared well. Reasonable life predictions were obtained for the open-hole data sets compared to the experimental data with a limited number of fatigue tests.

Segerfrojd et al. [31] investigated the fatigue life of aircraft structural joints. A total of ten different countersunk fastener types (Lockbolt, Hi-Lok, rivet, etc.) in open-hole specimens of 2024-T3 sheet of $2.5 \mathrm{~mm}$ thickness were subjected to the Mini-TWIST flight simulation loading with subsequent fractographic examination.

The equivalent initial flaw size distribution was developed from a probabilistic based equation of the form of Equation (1.1) and experimental data. Consistent sets of equivalent initial flaw size values were obtained by individual fastener systems when grouped into fairly large groups. In the Monte Carlo simulations, the crack growth rate analyses were coupled with a lognormal stochastic function to simulate the scatter.

Meaningful results were obtained when comparing the results of the threaded group with all other systems. In this case there was a large difference in equivalent initial flaw size between the two sets, with the threaded fasteners displaying the lower values. Monte Carlo simulations of all solid riveted specimens gave good agreement with experimental observations. It was shown that the influence of crack growth rate variation was not as significant as the initial flaw size distribution.

The main aim of Palmberg [32] in his study was to determine equivalent initial flaw sizes using four different methods; Paris' Law with two different sets of parameters, inverse 
hyperbolic tangent relation combined with a simple crack growth equation like equation (1.1), and Paris' Law combined with the strip yield model. An iterative scheme was developed to calculate the equivalent initial flaw size. The iteration was terminated when the difference between the computed and experimentally obtained number of cycles to failure was less than 100 cycles. Two types of 7010-T7651PL aluminum alloy specimens were considered, smooth unnotched and open-hole. Both specimens had a plate thickness of $6 \mathrm{~mm}$ and were subjected to constant amplitude loading at various stress levels with $R=0.1$.

The equivalent initial flaw size obtained showed a dependence on stress level, regardless of the method used, and appeared to be sensitive to the Paris' Law parameters. Also, the equivalent initial flaw size became very small, especially at the lower stress levels. Both Weibull and lognormal distributions seemed to fit the equivalent initial flaw size values better when compared to other distributions.

Newman et al. [33] conducted a study of the small crack effect for both 7075-T6 bare and LC9cs clad aluminum alloys in order to evaluate FASTRAN. In this study both experimental and analytical aspects of small and large crack growth were investigated. Large crack tests and analyses were conducted on center crack tension specimens, and small crack tests and analyses were conducted on single-edge notched tension fatigue specimens.

The authors concluded that the FASTRAN predicted crack growth rate trends for small cracks, created using constant amplitude and Mini-TWIST spectrum loading, were similar to those observed in tests, especially for the LC9cs clad alloy. The discrepancies observed for the 7075-T6 aluminum alloy were attributed to the plastic replica (acetone) method having an influence on small crack growth rates. Measured and predicted fatigue lives for constant 
amplitude and spectrum loading agreed quite well using a microstructural defect size, or the cladding layer thickness, as an initial flaw size.

Newman et al. [1] used FASTRAN-II to predict the fatigue lives of various metallic materials and loading conditions. The model was then used to calculate small and large crack growth rates, and to predict total fatigue lives, for notched and unnotched specimens made of two aluminum alloys, a titanium alloy, and a steel alloy under both constant amplitude and spectrum loading. Fatigue lives were calculated using the crack growth relations and microstructural features. Calculated and predicted fatigue lives for unnotched and notched specimens made of the two aluminum alloys compared well with test data for both constant amplitude and spectrum loading. Calculated fatigue lives for the titanium alloy were bounded by using initial crack sizes of 2 and $20 \mu \mathrm{m}$ in the life prediction method. Similarly, fatigue lives for notched specimens made of a high strength steel compared well with test data under constant amplitude and spectrum loading.

Laz and Hillberry [34] investigated the role of inclusions in fatigue crack formation. A test program was conducted using single-edge notched specimens of a 2024-T3 aluminum alloy. The distribution of inclusion sizes obtained from experimental observation was used as the stochastic input (Monte Carlo simulation) into a probabilistic life prediction model to predict the variability in experimental fatigue lives. The probabilistic model accurately predicted the shortest experimental fatigue lives and range of values under several loading conditions, but under predicted the longer lifetimes.

Gruenberg et al., [35] assessed the applicability of a probabilistic model for estimating fatigue life variability for 7075-T6 aluminum single-edge notched specimen tested under constant amplitude loading $\left(S_{\max }=120 M P a, R=0.01\right)$. A plastic replication procedure was 
used to identify crack nucleation sites and monitor crack growth. In agreement with previous studies, the initial fatigue cracks formed as surface cracks. The distribution of inclusion sizes $\left(0.2-45 \mu \mathrm{m}^{2}\right)$ within the material was used as an initial flaw size distribution for the FASTRANII input. The cumulative distributions for the fatigue lives using the numerical model were compared to the experimental data to assess the effectiveness of the numerical model. The model predicted failures well within the shorter life regime. The predicted lives were conservative, and in the shortest life region the predictions were within $15 \%$ of the observed lives. 


\section{CHAPTER 2}

\section{SCOPE OF WORK, ASSUMPTIONS, AND CHALLENGES}

\subsection{Scope of Work}

Test specimens of 2024-T3 clad aluminum alloy sheets (2.286 $\mathrm{mm}$ thick) with a variety of geometries were subjected to constant amplitude fatigue loads of various levels. Test specimen stress concentration factors are functions of the specimen geometry. They are usually determined through finite element analysis. Stress concentration factors associated with the various specimens of study ranged from 1.000 to 7.671. Altogether five specimen types with various levels of applied fatigue loading were studied during this investigation.

\subsection{Experimental Data}

Experimental $S-N$ data were generated as a result of the research project of Reference

[3]. The following specimen geometries were considered during the research program:

a) Smooth unnotched specimen

b) Open-hole specimen

c) $K_{t}=3$ double-edge $V$-notched specimen

d) $K_{t}=4$ double-edge $V$-notched specimen

e) $K_{t}=5$ double-edge $V$-notched specimen

All the above specimens were made from a 2024-T3 clad aluminum alloy and each type produced a stress concentration factor that was different from that of the other types of the study. The angle of the V-notch for all of the notched specimen types was 60 degrees. The stress concentration factors for all specimens were computed with finite element analyses and are 
presented by specimen type in Table 2.1. Stress concentration factors were determined from 2-D quarter symmetry finite element models. The element type used was the CPS8 element which is an 8-noded plane stress quadrilateral element with three translational degrees of freedom at each node. Each of the finite element models was subjected to a remote axial load that produced a uniform remote stress of $S_{\text {app }}=1.0 M P a$. Due to symmetry, only one-quarter of the specimen was modeled and analyzed. The size of the mesh is given in Table 2.1

The stress concentration factor, $K_{\mathrm{tg}}$, is expressed in terms of remote (gross) stress, rather than the net section stress. The geometric specifications of each of the specimen types are given in Figures 2.1 through 2.5 below.

The smooth unnotched specimen was treated as a rectangular plate with a width equal to the uncracked ligament length. A rectangular plate has a $K_{\mathrm{tg}}$ of 1.0. The theoretical basis of this assumption will be discussed in Chapter 4.0 of this document.

\section{Table 2.1}

Finite element mesh details and resulting stress concentration factors for the five types of specimens of the study

\begin{tabular}{|c|c|c|c|}
\hline Specimen type & $K_{\mathrm{tg}}$ & Number of elements & Number of nodes \\
\hline Smooth unnotched & $1.000^{*}$ & 434 & 1445 \\
\hline Open-hole & 3.036 & 1216 & 3809 \\
\hline$K_{\mathrm{t}}=3$ & 5.165 & 5314 & 16261 \\
\hline$K_{\mathrm{t}}=4$ & 7.013 & 4121 & 12702 \\
\hline$K_{\mathrm{t}}=5$ & 7.671 & 3908 & 12063 \\
\hline
\end{tabular}

Note: * The smooth unnotched specimen can be treated as a rectangular plate with $K_{\mathrm{tg}}$ of 1.000 . Refer to Chapter 4. 


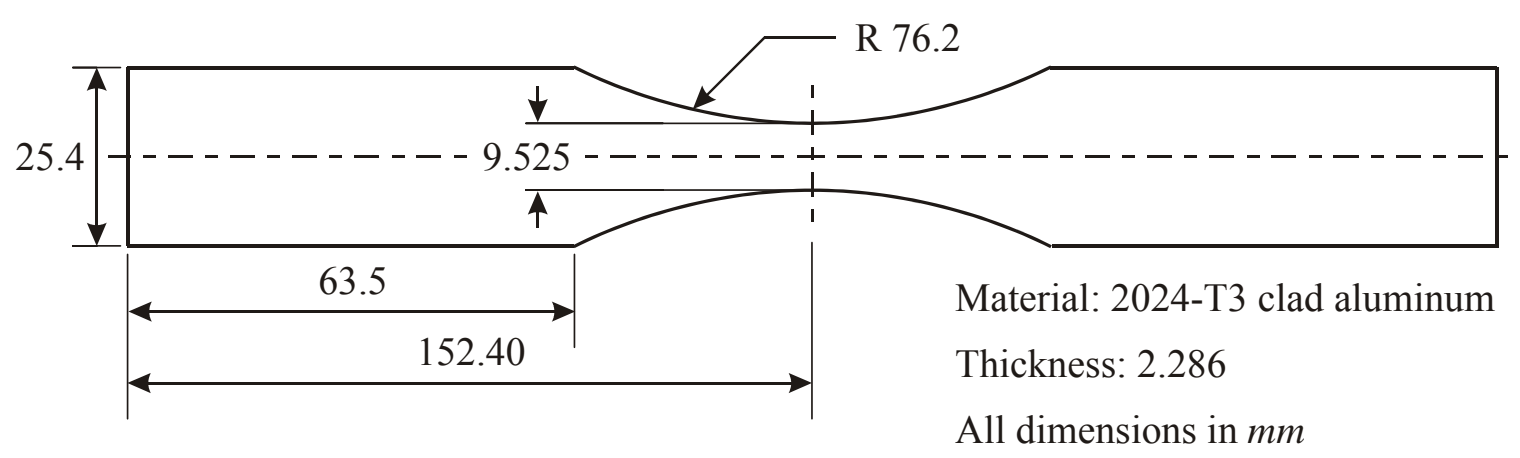

Figure 2.1 Geometric details of the smooth unnotched specimen

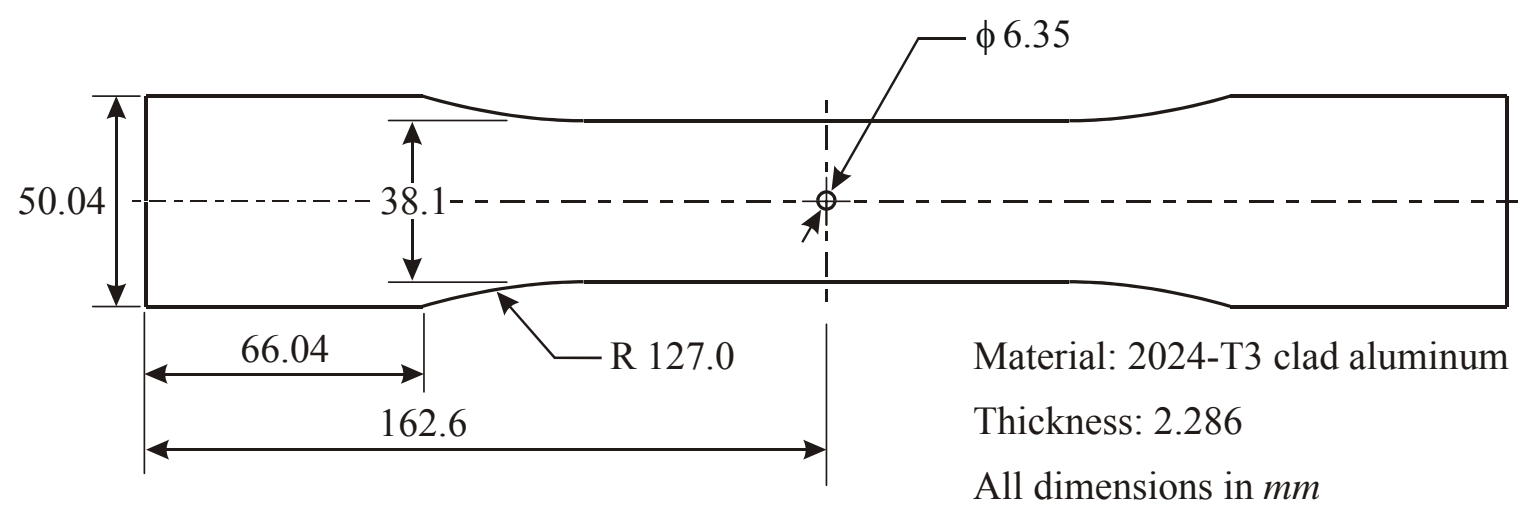

Figure 2.2 Geometric details of the open-hole specimen

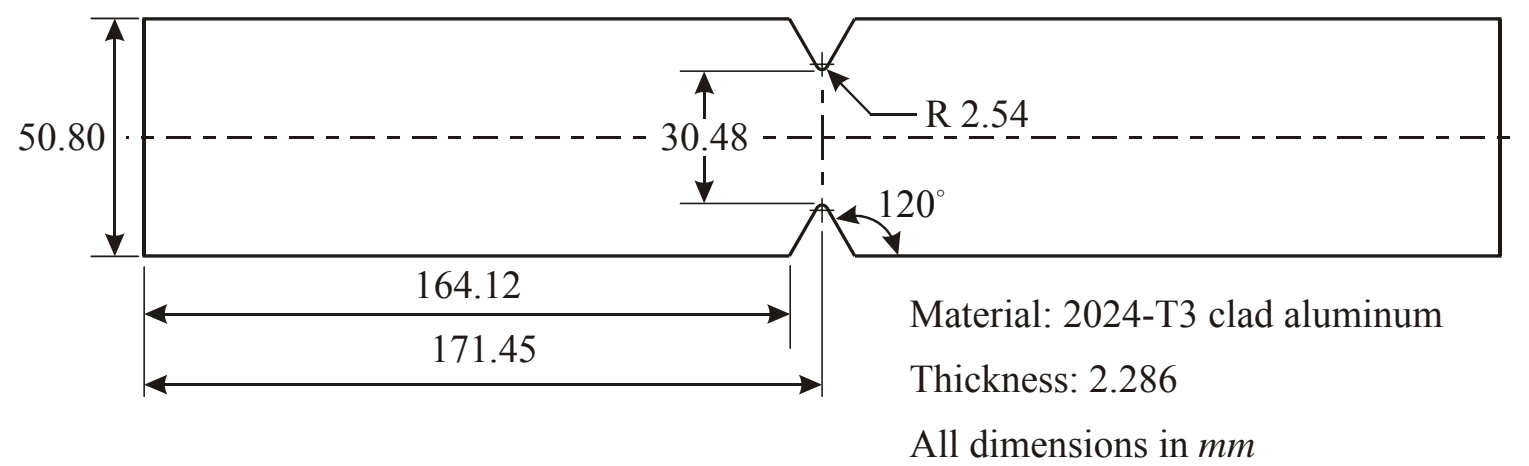

Figure 2.3 Geometric details of the $K_{\mathrm{t}}=3$ specimen 


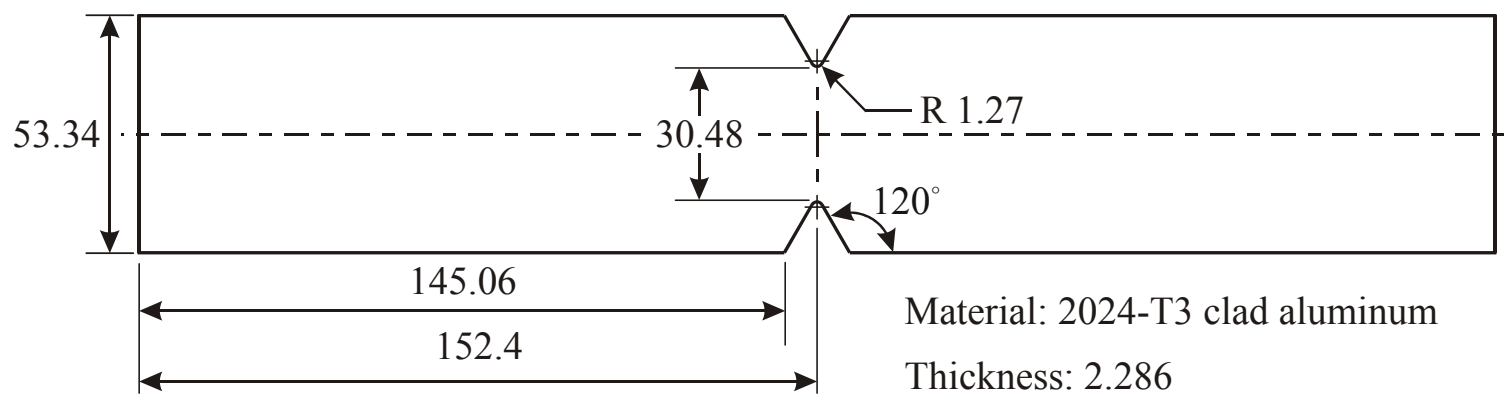

All dimensions in $\mathrm{mm}$

Figure 2.4 Geometric details of the $K_{\mathrm{t}}=4$ specimen

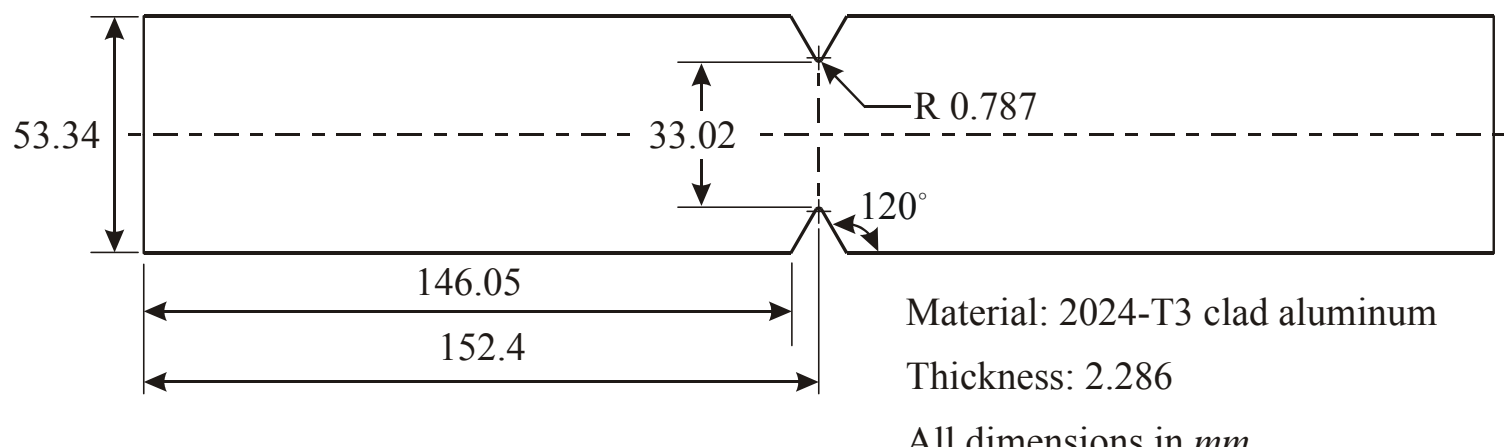

Figure 2.5 Geometric details of the $K_{\mathrm{t}}=5$ specimen

Figures 2.6 through 2.10 contain the $S-N$ plots obtained experimentally for each of the types of specimens defined above. In these plots the maximum value of the stress, $S_{\max }$, associated with the amplitude of the constant amplitude loading was plotted against $N$, the number of cycles to failure or measured fatigue life. For the smooth unnotched specimen, the data were obtained for a mean value of stress, $S_{\text {mean }}$ of $144.790 \mathrm{MPa}$ and $S_{\max }$ of 344.738 , 282.685, 241.316, 210.290, and 206.843 $\mathrm{MPa}$. Here the $S_{\text {mean }}$ and $S_{\max }$ values are not the remotely applied actual loads but stress values which were normalized to the width at the neck of the specimen. Equation (4.2) provides the expression for the normalized stress values as a 
function of the applied load. For the open-hole and the double-edge notched specimens $S-N$ data were obtained for two different mean stress levels, $S_{\text {mean }}$ of 20.684 and $41.369 M P a$. $S-N$ data for the open-hole specimens were measured for the combinations of $S_{\text {mean }}$ of 20.684 $M P a$ and $S_{\max }$ of $124.106,82.737,68.948$, and $63.777 \mathrm{MPa}$ and for the combinations of $S_{\text {mean }}$ of 41.369 $\mathrm{MPa}$ and $S_{\max }$ of $124.106,86.184$, and 79.290 MPa. $S-N$ data for the $K_{\mathrm{t}}=3$ specimens were recorded for the loading combinations of $S_{\text {mean }}$ of $20.684 \mathrm{MPa}$ and $S_{\max }$ of 68.948 and 48.263 MPa and for the combinations of $S_{\text {mean }}$ of $41.369 \mathrm{MPa}$ and $S_{\max }$ of 96.527 and $62.053 \mathrm{MPa}$. For the $K_{\mathrm{t}}=4$ specimens, $S-N$ data were obtained for the combinations of $S_{\text {mean }}$ of $20.684 \mathrm{MPa}$ and $S_{\text {max }}$ of $62.053,37.921$, and $32.061 \mathrm{MPa}$ and for the combinations of $S_{\text {mean }}$ of 41.369 $\mathrm{MPa}$ and $S_{\max }$ of 75.842, 55.158, and 51.711 $\mathrm{MPa}$. Similarly, for the $K_{\mathrm{t}}=5$ specimens, $S-N$ data were obtained for the combinations of $S_{\text {mean }}$ of $20.684 \mathrm{MPa}$ and $S_{\max }$ of $62.053,48.263,34.474$, and $32.750 \mathrm{MPa}$ and for the combinations of $S_{\text {mean }}$ of $41.369 \mathrm{MPa}$ and $S_{\max }$ of $75.842,62.053,53.434$, and $51.711 \mathrm{MPa}$. The $S-N$ data for the smooth unnotched specimens were obtained for higher stress values than those for either the open-hole or the double-edge notched specimens. The $K_{\mathrm{t}}=4$ specimens had two cases of runouts at a $S_{\text {mean }}$ of $20.684 \mathrm{MPa}$. 


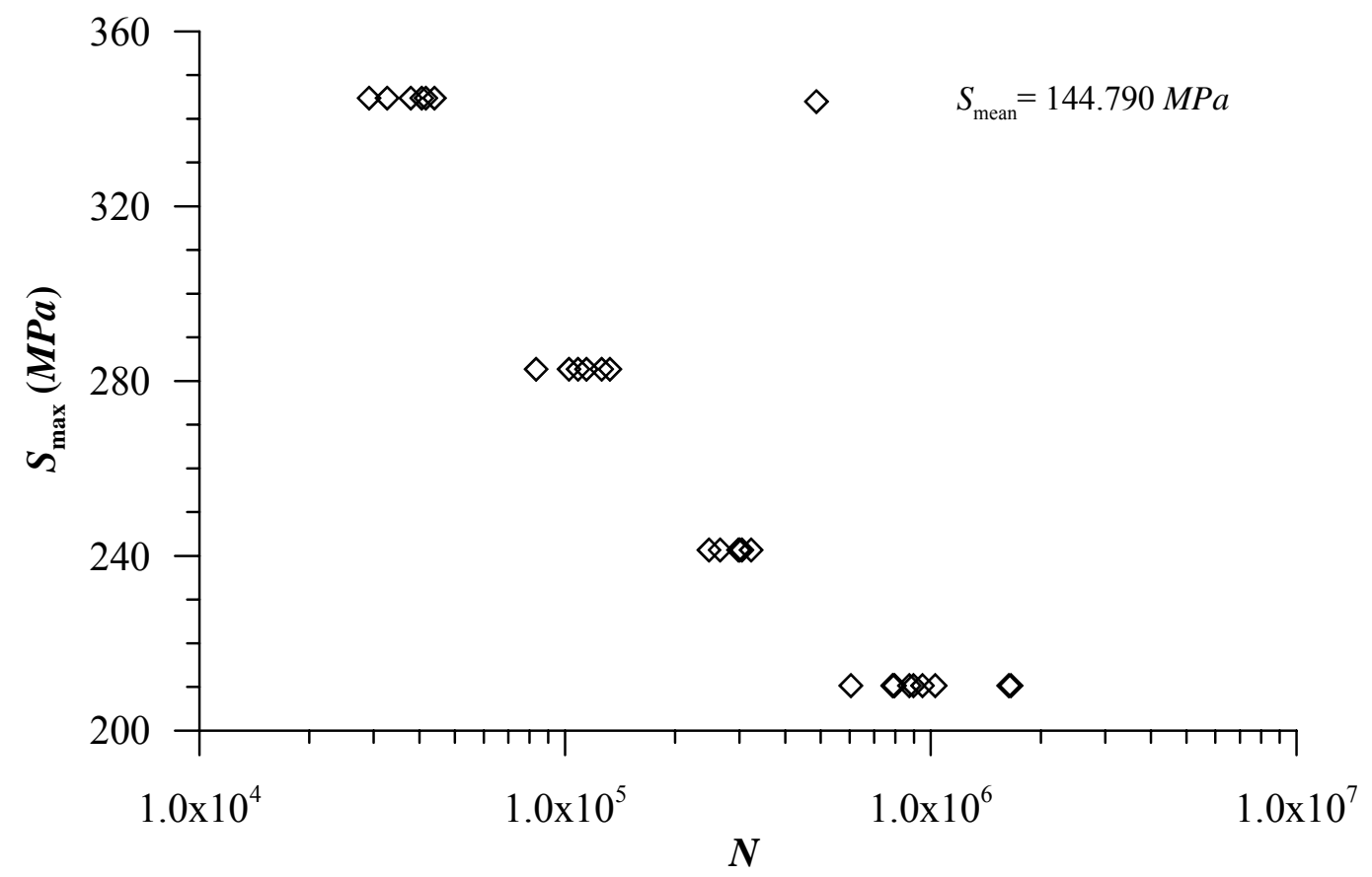

Figure 2.6 $S-N$ data from smooth unnotched specimens

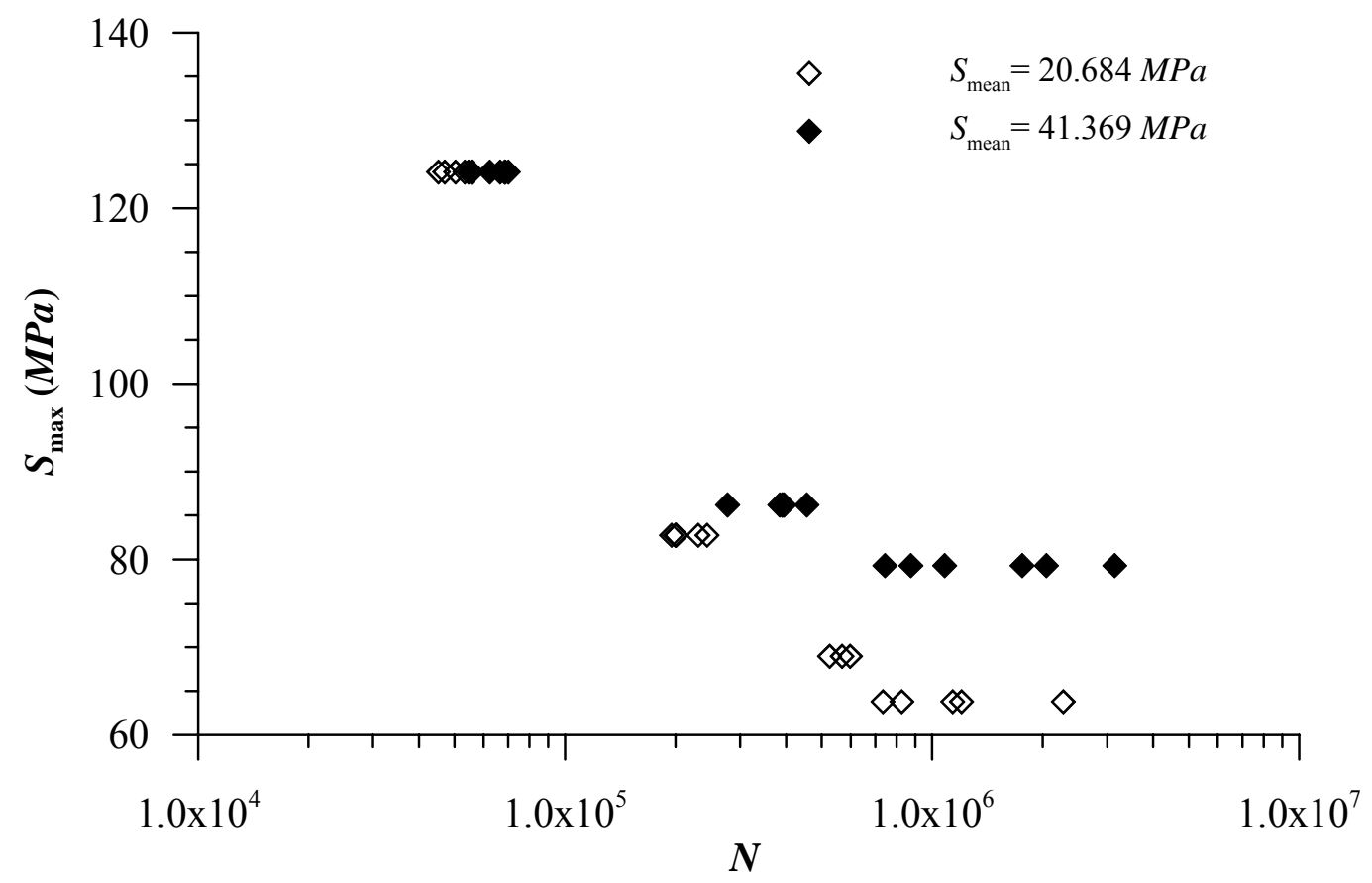

Figure 2.7 $S-N$ data from open-hole specimens 


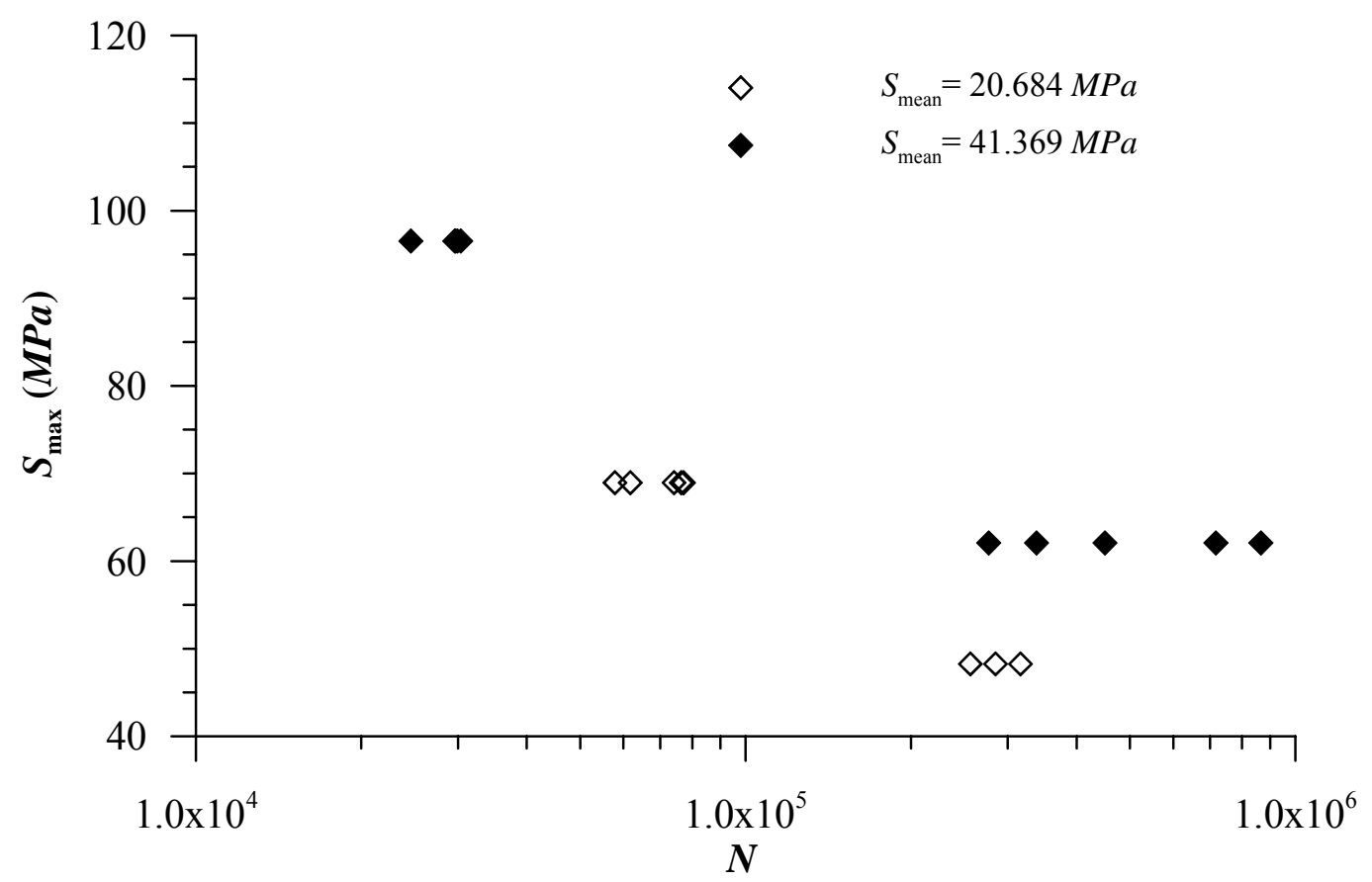

Figure 2.8 $S-N$ data from $K_{\mathrm{t}}=3$ specimens

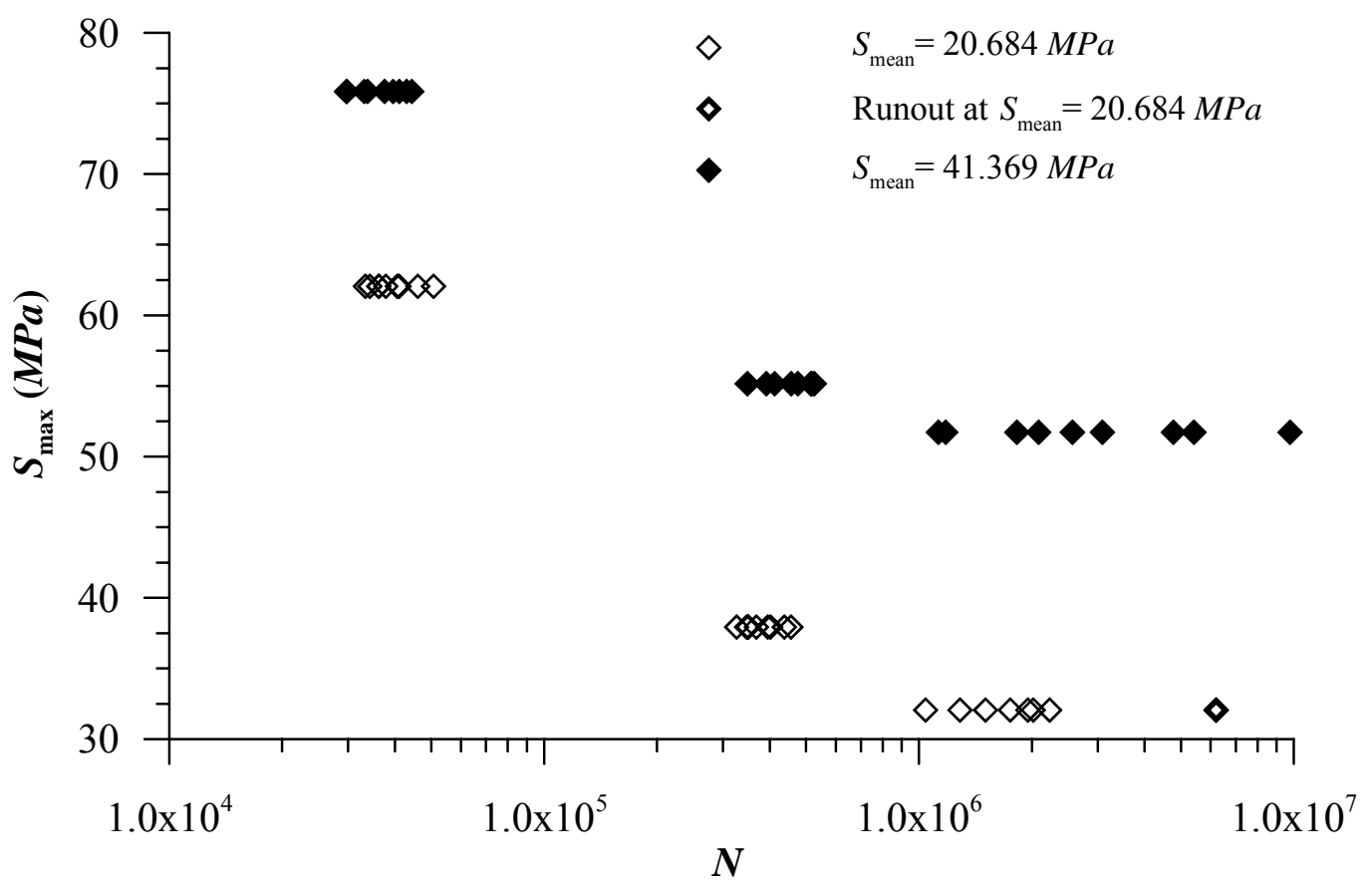

Figure 2.9 $S-N$ data from $K_{\mathrm{t}}=4$ specimens 


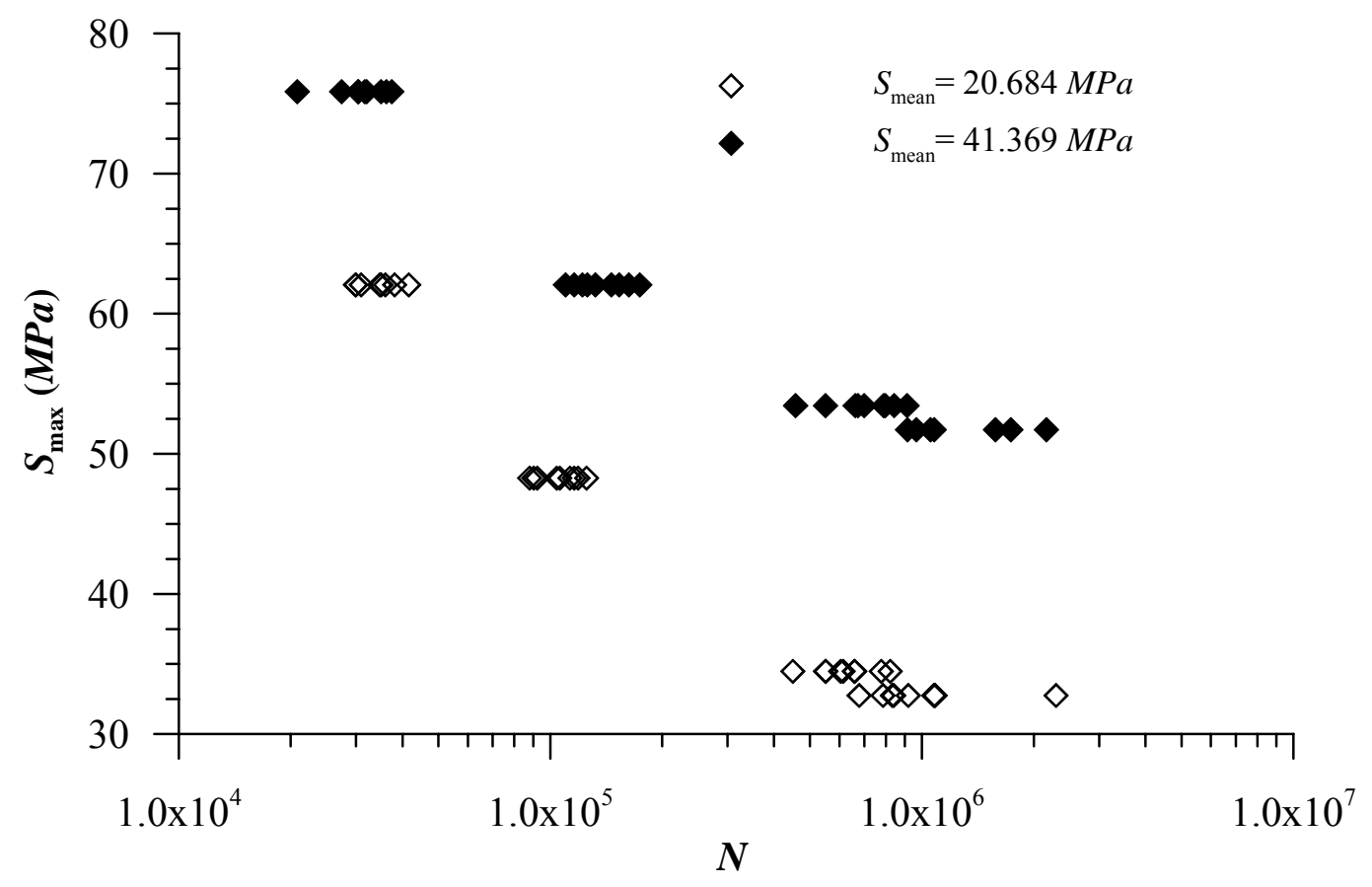

Figure 2.10 $S-N$ data from $K_{\mathrm{t}}=5$ specimens

\subsection{Probabilistic Model Assumptions}

The following assumptions were incorporated into the probabilistic model of the research study of this document:

a) Cracks form from a single initial flaw. Based on a great deal of previous scanning electron microscope (SEM) observations, researchers have concluded that most cracks initiate as either corner or surface flaws. Two kinds of initial flaw configurations were considered for this study; the single quarter circular corner crack and the single semicircular surface crack. This was done by setting $a_{0}=c_{0}$ for the crack configuration geometries illustrated in Figures 2.11 and 2.12.

b) Crack formation occurs from one planar side of the specimen and not from both sides.

c) The height of the nucleating defect was assumed to be $3 \mu \mathrm{m}$. 


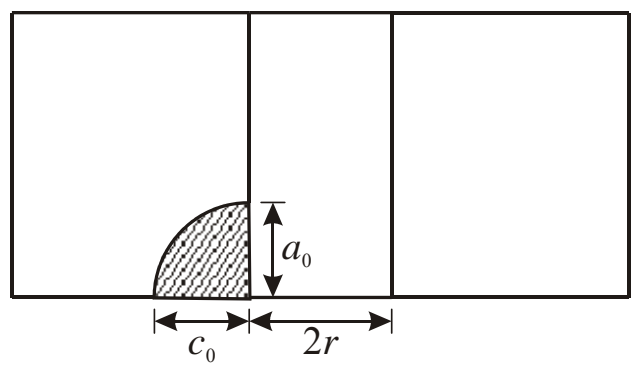

(a)

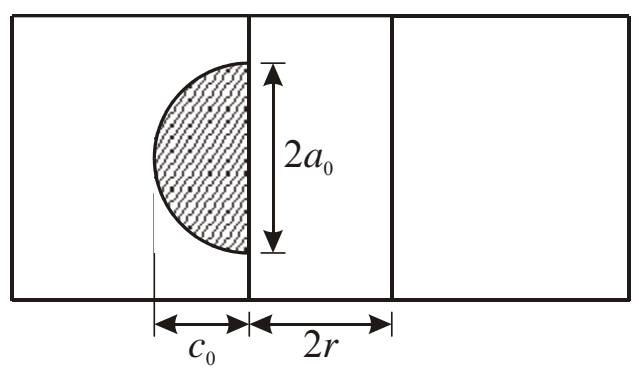

(b)

Figure 2.11 Initial (a) corner and (b) surface crack configuration for open-hole specimens

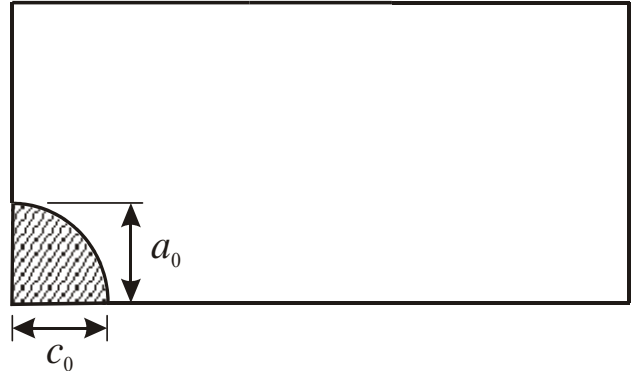

(a)

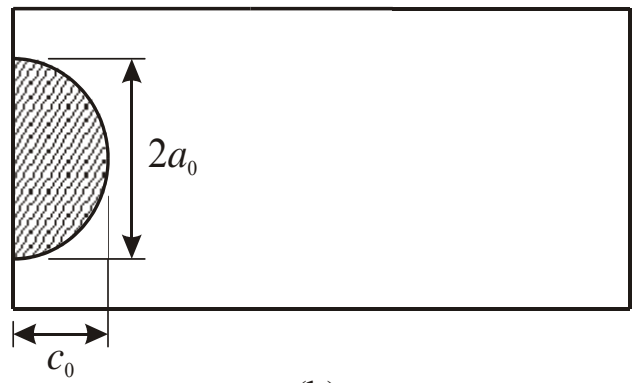

(b)

Figure 2.12 Initial (a) corner and (b) surface crack configuration for smooth unnotched specimens and for the $K_{\mathrm{t}}=3, K_{\mathrm{t}}=4$, and $K_{\mathrm{t}}=5$ specimens

d) The crack propagation path was assumed to be parallel to and coincident with the symmetric plane of the specimens.

e) Nucleation life was neglected. Life is defined from crack size equal to equivalent initial flaw size to failure.

f) Equivalent initial flaw sizes were determined from the measured fatigue life of each specimen.

\section{$2.4 \quad$ Challenges}

There were some basic problems with the equivalent initial flaw size concept. First, the derived equivalent initial flaw sizes often lack credibility because such flaws cannot be directly 
verified by present non-destructive inspection (NDI) technique. The equivalent initial flaw sizes were derived for different design variables and loading spectra as discussed in Section 1.4 because they could not be verified as generic material property.

The applicability and limits of linear elastic fracture mechanics principles for predicting crack growth in the small crack size regime have not been well founded. Most previous investigators have calculated equivalent initial flaw size from the number of cycles corresponding to assumed initial crack size of about $0.762 \mathrm{~mm}$, known as the time-to-crackinitiation. However, in this study equivalent initial flaw size was determined based upon the measured fatigue life, $N$. A crack size of $0.762 \mathrm{~mm}$ is relatively small when compared to the final crack size at specimen failure. Between the time of initial crack length and final failure, the plastic zone size has become more pronounced and has a significant influence on the material response. Reference [3] only provided measured fatigue lives and therefore equivalent initial flaw sizes were determined using measured fatigue life data. Getting time-to-crack-initiation data from laboratory experiments could be expensive because it requires special crack growth monitoring gage. Reference [3] also did not report crack growth history, or the final crack size. If made available crack growth history data could be superimposed on the back extrapolation curve from FASTRAN-II to validate equivalent initial flaw size calculations. Equivalent initial flaw size could be evaluated more accurately if final crack size were used instead of measured fatigue life. The combination of final crack size and crack growth history would allow for implementation of fine adjustment that could perhaps improve the calculation of the equivalent initial flaw size. 


\section{CHAPTER 3}

\section{FINITE ELEMENT CRACK MODELING}

\subsection{Introduction}

One of the required input parameters for the FASTRAN-II code is the stress intensity factor. FASTRAN-II contains a vast library of likely configurations and loads, but for those configurations that are not built into FASTRAN-II, the user must provide stress intensity factors for various crack sizes and configurations in tabular form in order to conduct crack growth analyses. There are various computational methods that can be used to compute stress intensity factors, but the two most notable methods are the finite element method and the boundary element method. The main disadvantage of the finite element method is that the entire domain of the subject must be discretized while the boundary element method involves discretization only of the boundary of the subject. However, the boundary element method generally leads to a nonsymmetric, fully populated system of equations, unlike the finite element method which yields a symmetric, banded system of equations. In this study the finite element method was used to compute the stress intensity factors for the various crack sizes and geometries of the project.

In this chapter we discuss the fundamental formulation and usage of finite element method techniques to calculate the linear elastic fracture mechanics stress intensity factors and energy release rates. The chapter describes:

a) the procedure employed to model the elastic crack front singularity,

b) the computational process employed to compute the stress intensity factors for 2-D and 3-D problems. 


\subsection{Singular Finite Elements}

In general, the fundamental difficulty associated with modeling crack extension is the representation of the singular crack-tip stress and strain fields predicted by linear elastic fracture mechanics theory using conventional finite elements. Even if the overall mesh of elements is refined, the finite element solution will initially tend to converge, but eventually diverges. Chan et al. [36] identified this problem in the initial development stages of the finite element method. Numerous investigators approached this problem by introducing singularity function formulations in the finite element or stress intensity factors as nodal variables (e.g., Byskov [37]; Tracey [38]; Tong et al. [39]; Papaioannou et al. [40]; Atluri et al. [41]; and Benzley [42]) with reasonable success. However, these elements are not found in most commercial codes.

The introduction of the quarter-point element was a significant development in the application of the finite element method for linear elastic fracture mechanics problems. The quarter-point element concept was the result of simultaneous and independent work by both Henshell and Shaw [44] and Barsoum [45]. These researchers demonstrated that the proper crack-tip displacement, stress, and strain singularity fields can be modeled by locating the element's mid-side node one quarter of the way between crack-tip and far end nodes, as illustrated in Figure 3.1. 


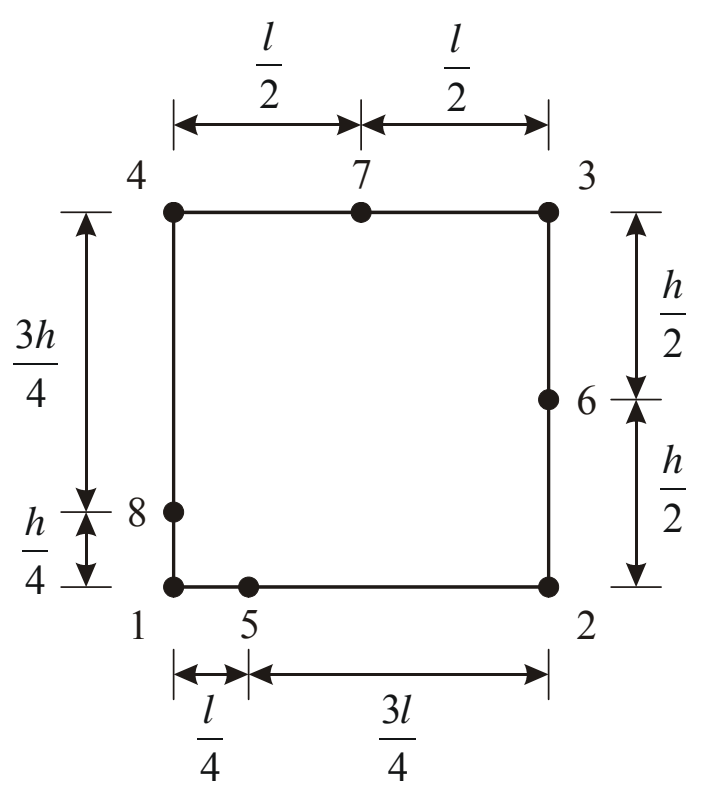

(a)

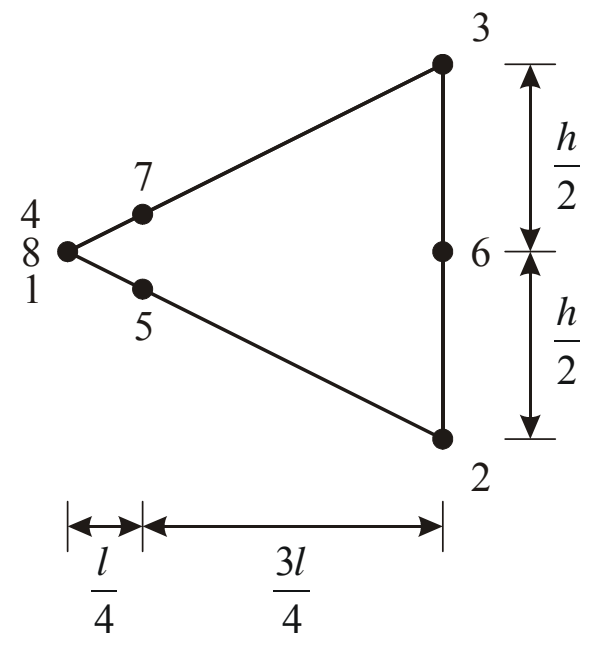

(b)

Figure 3.1 (a) Quadrilateral and (b) collapsed quadrilateral quarter-point elements (Courtesy of Ingraffea and Wawrzynek [43])

The quadrilateral quarter-point element described by Henshell and Shaw [44] is depicted in Figure 3.1(a) while Figure 3.1(b) illustrates Barsoum's [45] proposal of collapsing one edge of the element at the crack-tip. The quarter-point element was a significant milestone in the development of finite element procedures for linear elastic fracture mechanics, because minimal numerical processing was required with the usage of these elements to model the crack-tip in finite element codes. The remainder of this section will focus exclusively on various forms of quarter-point elements.

\subsection{1-D Quarter-Point Elements}

The quarter-point concept can be best illustrated with a 1-D element. While such an element has no use in practice, it does simplify the algebraic derivations and the same principals 
can be applied to 2-D and 3-D elements without any loss in generality. A 1-D quadratic order element is illustrated in Figure 3.2. Figure 3.2(a) shows the parametric space of the element while Figure 3.2(b) shows the element in the Cartesian space, with the location of the center node controlled by the value of the parameter $\omega$ and the "crack-tip" is located at $r=0$.

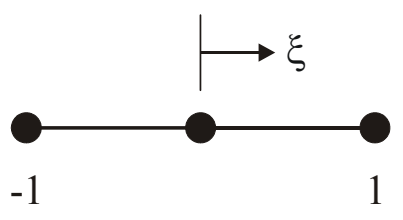

(a)

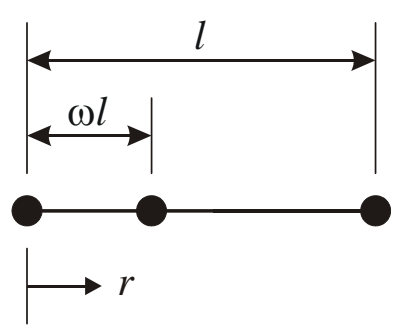

(b)

Figure 3.2 The 1-D quadratic element in (a) parametric space and (b) Cartesian space (Courtesy of Ingraffea and Wawrzynek [43])

Define $u_{i}$ to be the nodal axial displacement of the $i^{\text {th }}$ node. Therefore, the displacement, $u$, at any point within the element can be determined using standard second-order Langrangian shape functions, $N_{i}=N_{i}(\xi)$, and nodal displacements.

$$
\begin{gathered}
u=\sum_{i=1}^{3} N_{i} u_{i} \\
u=\frac{1}{2} \xi(\xi-1) u_{1}+\left(1-\xi^{2}\right) u_{2}+\frac{1}{2} \xi(\xi+1) u_{3}
\end{gathered}
$$

or, regrouping in powers of $\xi$,

$$
u=u_{2}+\frac{1}{2}\left(u_{3}-u_{1}\right) \xi+\left[\frac{1}{2}\left(u_{1}+u_{3}\right)-u_{2}\right] \xi^{2}
$$

Using an isoparametric formulation, the same shape functions are used to interpolate the geometry of the element: 


$$
r=\sum_{i=1}^{3} N_{i} r_{i}=\omega l+\frac{1}{2} l \xi+l\left(\frac{1}{2}-\omega\right) \xi^{2}
$$

Consider the case where the center node is located at the midpoint of the element. Substituting $\omega=1 / 2$ into equation (3.3), $\xi$ becomes,

$$
\xi=2 \frac{r}{l}-1
$$

Substituting this expression for $\xi$ into equation (3.2) yields the expected quadratic expression for the axial displacement at arbitrary $r$ :

$$
u=u_{1}+\left(-3 u_{1}+4 u_{2}-u_{3}\right) \frac{r}{l}+2\left(u_{1}-2 u_{2}+u_{3}\right) \frac{r^{2}}{l^{2}}
$$

Differentiating this expression with respect to $r$ yields the expected linear expression for the axial strain in the element at arbitrary location, $r$ :

$$
\varepsilon=\frac{d u}{d r}=\left(-3 u_{1}+4 u_{2}-u_{3}\right) \frac{1}{l}+4\left(u_{1}-2 u_{2}+u_{3}\right) \frac{r}{l^{2}}
$$

Assuming linearly elastic conditions, the stresses are linearly related to the strains so the stress distribution will be linear in $r$ as well.

Now consider the case where the intermediate node is moved to the quarter-point position. After substituting $\omega=1 / 4$ into equation (3.3), $\xi$ becomes:

$$
\xi=2 \sqrt{\frac{r}{l}}-1
$$

Substituting this expression for $\xi$ into equation (3.2) and differentiating yields the following expressions for the axial displacement and strain in the element at arbitrary $r$ :

$$
u=u_{1}+2\left(u_{1}-2 u_{2}+u_{3}\right) \frac{r}{l}+\left(-3 u_{1}+4 u_{2}-u_{3}\right) \sqrt{\frac{r}{l}}
$$




$$
\varepsilon=\frac{d u}{d r}=2\left(u_{1}-2 u_{2}+u_{3}\right) \frac{1}{l}+\frac{1}{2}\left(-3 u_{1}+4 u_{2}-u_{3}\right) \frac{1}{\sqrt{r l}}
$$

The expression for the axial strain contains a constant term and a singular term that varies as a

function of $r^{-1 / 2}$; similar to the form of the lead term in the linear elastic fracture mechanics stress and strain expansions near the crack-tip singularity.

\subsection{2-D Quarter-Point Elements}

The quadrilateral quarter-point element shown in Figure 3.1(a) is less frequently used in practice compared to the collapsed quadrilateral version, Figure 3.1(b) because fewer of these elements can be placed conveniently around a crack-tip. With fewer elements, the (trigonometric) circumferential variation of the stress and displacement fields about a crack-tip may be less accurately represented. Consequently, the collapsed quadrilateral element is the more popular. As a general rule of thumb the minimum number of elements required to model a 2-D crack-tip is between eight and ten elements. Most commercially available finite element codes come with the collapsed quadrilateral quarter-point elements for crack-tip modeling. Figure 3.3 illustrates a 2-D crack-tip modeled with eight collapsed quadrilateral quarter-point elements. 


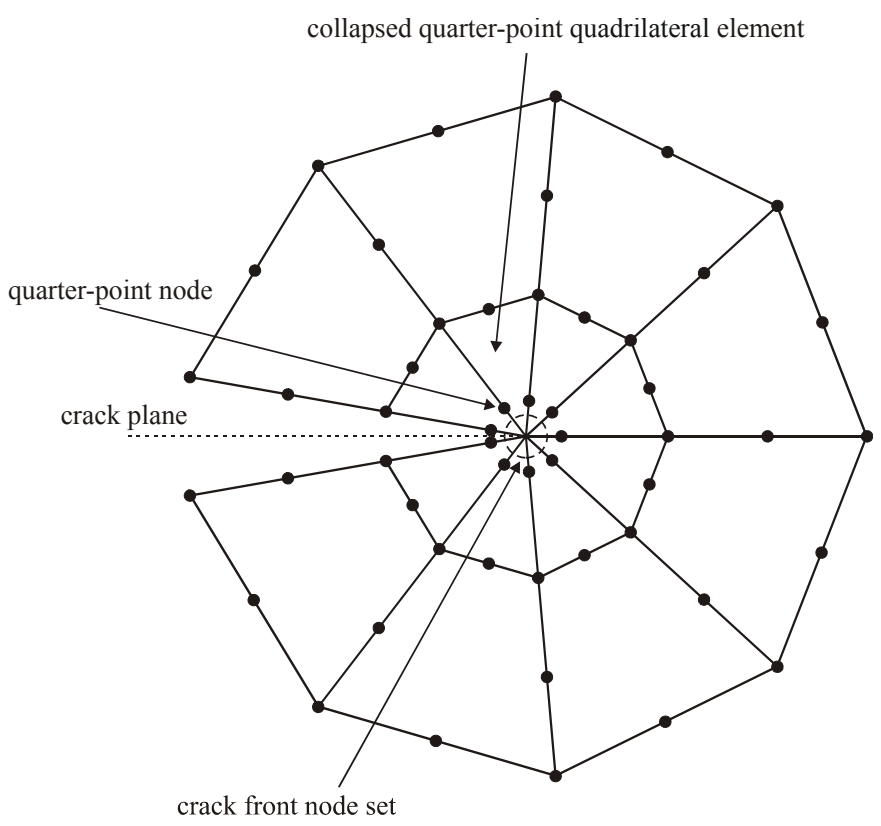

Figure 3.3 Crack-tip modeled with eight collapsed quadrilateral quarter-point elements

Both Barsoum [46] and Freese and Tracey [47] showed that when the side node (node 6 of Figure 3.1(b)) on the edge opposite the crack-tip is placed at the midpoint of a straight line between the two corner nodes (nodes 2 and 3) for the collapsed quadrilateral element, the $r^{-1 / 2}$ singularity condition shown in equation (3.9), holds along paths of constant parametric coordinate, $\eta$. In Cartesian space lines of constant $\eta$ map into straight rays emanating from the crack-tip as illustrated in Figure 3.4(b). When node 6 is placed at eccentric midpoint between nodes 2 and 3 as shown in Figure 3.4(c), lines of constant $\eta$ map into quadratic curves in the Cartesian space. 


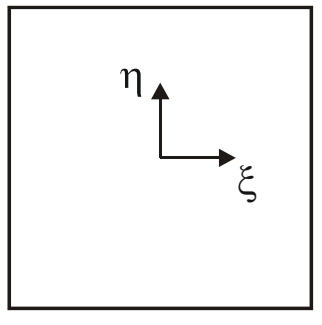

(a)

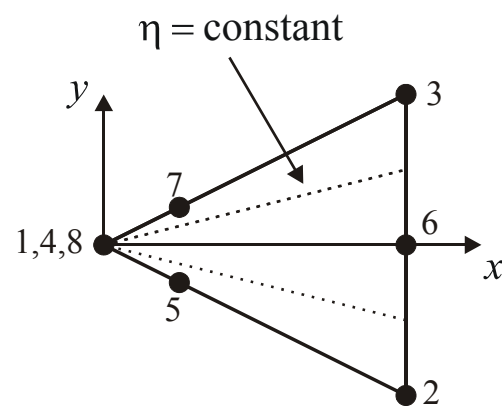

(b)

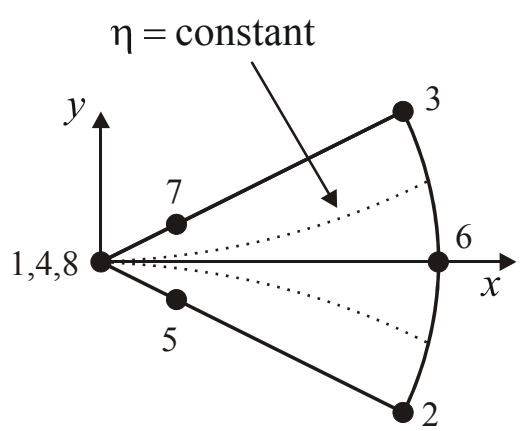

(c)

Figure 3.4 (a) Parametric space of a quadrilateral element; mapping of constant $\eta$ lines in Cartesian space when node 6 is placed (b) midpoint and (c) eccentric midpoint between far side nodes 2 and 3 (Courtesy of Ingraffea and Wawrzynek [43])

\subsection{3-D Quarter-Point Elements}

The 3-D quarter point element is created by extruding the 2-D element along the crack front. Barsoum [45] considered the usage of a collapsed 20-noded hexahedral element, which is actually a natural extension of the collapsed 8-noded quadrilateral. The elements were straightsided in which three of the element faces were rectangles.

Hussain et al. [48], Manu [49], and Koers [50] considered collapsed 20-noded elements with curved crack fronts. Manu [49] also presented conditions on nodal positions that must be observed in order to ensure $r^{-1 / 2}$ singularity on all rays emanating from the crack front. With respect to the collapsed brick element of Figure 3.5, these constraints are

$$
\begin{gathered}
x_{1}=x_{4}=x_{12} \\
x_{17}=x_{20} \\
x_{5}=x_{8}=x_{16}
\end{gathered}
$$




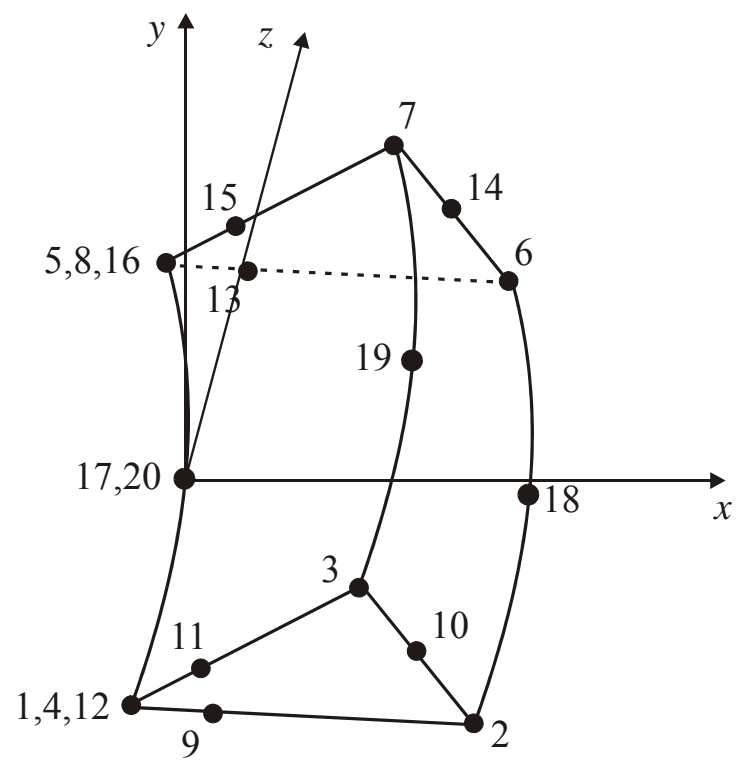

Figure 3.5 The collapsed, 20-noded brick, quarter-point element (Courtesy of Ingraffea and Wawrzynek [43])

$$
\begin{aligned}
& x_{10}=\frac{1}{2}\left(x_{2}+x_{3}\right) \\
& x_{14}=\frac{1}{2}\left(x_{6}+x_{7}\right) \\
& x_{9}=\frac{1}{4}\left(x_{1}+x_{2}\right) \\
& x_{11}=\frac{1}{4}\left(x_{1}+x_{3}\right) \\
& x_{13}=\frac{1}{4}\left(x_{5}+x_{6}\right) \\
& x_{15}=\frac{1}{4}\left(x_{5}+x_{7}\right)
\end{aligned}
$$

In addition, the less obvious conditions:

$$
\begin{aligned}
& x_{18}=\frac{1}{2}\left(-x_{1}+x_{2}-x_{5}+x_{6}+2 x_{17}\right) \\
& x_{19}=\frac{1}{2}\left(-x_{1}+x_{3}-x_{5}+x_{7}+2 x_{17}\right)
\end{aligned}
$$

must be enforced. Similar conditions hold for the $y$ and $z$ coordinates of the nodes. 


\subsection{Crack Modeling}

Mode I stress intensity factor, $K_{\mathrm{I}}$ is a required input to the FASTRAN-II code, which has a built-in menu of seventeen predefined crack configurations. The smooth unnotched specimens, and the $K_{\mathrm{t}}=3, K_{\mathrm{t}}=4$ and $K_{\mathrm{t}}=5$ specimens were not members of the predefined crack menu of FASTRAN-II and therefore it was necessary to use finite element analyses to obtain the stress intensity factor for those cases. In this study all cracks were assumed to form either as a single quarter circular corner crack, or as a single semicircular surface crack. Consequently, stress intensity factors were computed for three configurations; single corner crack, single surface crack, and through crack configurations.

Crack modeling using finite element methods can be a very complicated process. A partthrough crack must be modeled with a 3-D mesh, whereas a through crack can be meshed with 2D elements provided the geometry of the object is appropriate. The capability to model the crack-tip, or crack front, with most commercial finite element software is limited. In this study three software packages (MSC.Patran 2004 [51], ZENCRACK Version 7.3e [52], and ABAQUS Version 6.5 [53]) were utilized in order to calculate stress intensity factors. The flowchart of Figure 3.6 describes the order and function of each code involved in the process. 


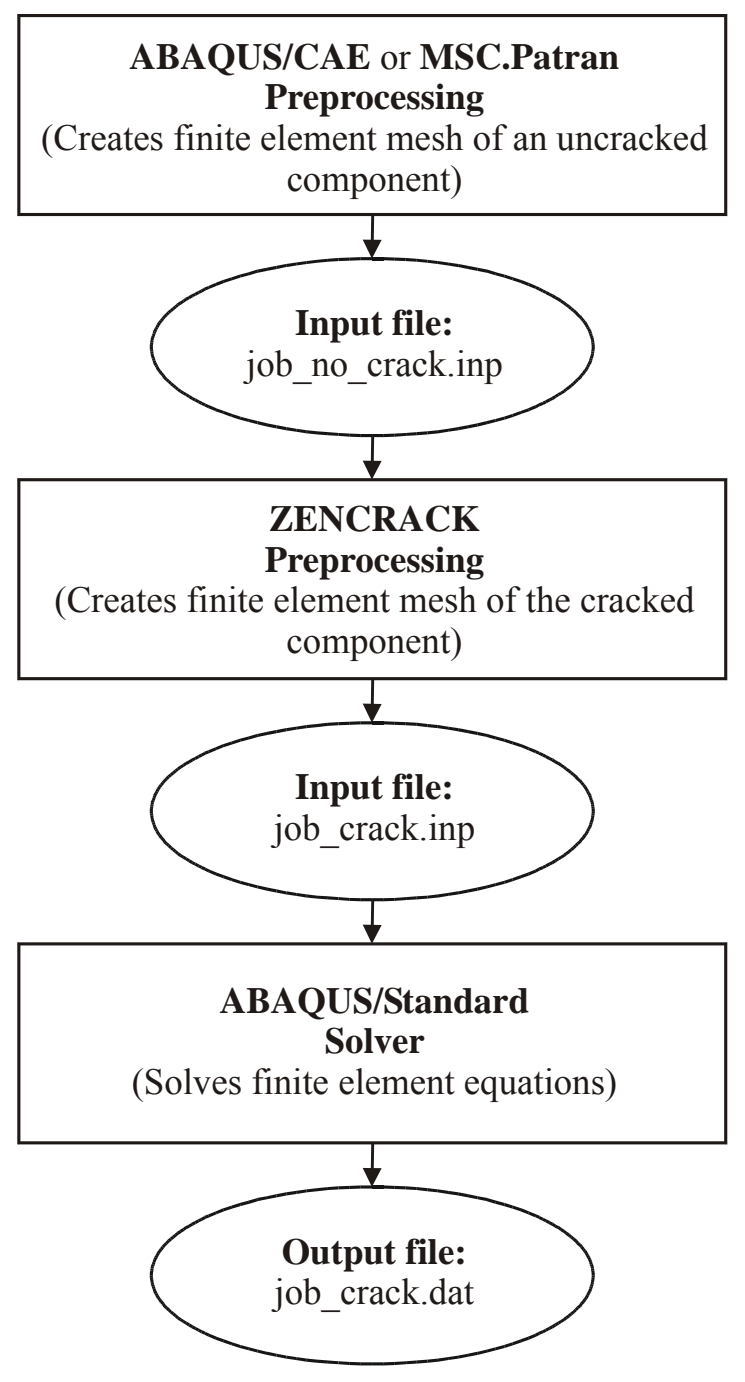

Figure 3.6 Flow chart of the combination of commercial codes used to determine the stress intensity factors of the specimens

The finite element preprocessing for this study was done using MSC.Patran, a general purpose computer aided engineering code for interactive, 3-D modeling and visualization of engineering analysis results. The output product of the MSC.Patran preprocessing is an ABAQUS input file (.inp) for the component with no crack.

ZENCRACK is a state-of-the-art software tool for 3-D fracture mechanics simulation. A ZENCRACK simulation requires an uncracked 3-D finite element mesh of the component of 
interest. This can be generated using a standard preprocessing tool along with standard finite element software. With this uncracked 3-D mesh input ZENCRACK creates a mesh for the "cracked" case by inserting one or more user specified cracks into the mesh. This is accomplished by executing ZENCRACK. A minimum of two input files is required for any ZENCRACK analysis: the uncracked mesh file, and the ZENCRACK input file. The ZENCRACK input file is an ASCII file of additional information. The file must have extension ".zcr" and be located in the same directory as the uncracked mesh file. The ZENCRACK input file contains keyword and data lines to define the crack-related requirements for the analysis such as name of the uncracked mesh file, type of crack-block to be used (corner, surface and through), the element number in the uncracked mesh in which the crack-block is going to be substituted, the orientation of the crack front, the crack length, $c$, and crack depth, $a$. Once ZENCRACK is executed it creates .inp file with a cracked mesh. This mesh is submitted to the finite element solver code for structural analysis. The finite element solver used in this study was ABAQUS/Standard. ABAQUS/Standard provides traditional implicit finite element analyses for a range of contact and nonlinear material options. A standard feature of the ABAQUS/Standard code is the capacity to compute the stress intensity factor distributions along the specified crack front(s) of the body. Quadrilateral elements were used for the 2-D analyses and hexahedral elements were used for the 3-D analyses.

\subsection{Stress Intensity Factor Extraction}

Under linear elastic fracture mechanics assumptions, the stress, strain, and displacement fields in the region near the crack-tip can be determined using the stress intensity factor. Therefore, essential to the use of the finite element method for linear elastic fracture mechanics 
analyses is the extraction of accurate stress intensity factors from the results of finite element statistic load analyses. Several methods are available for extracting stress intensity factors. These include the displacement correlation, the virtual crack extension, the modified crack closure integral, and the $J$-integral techniques. The ABAQUS/Standard uses the $J$-integral technique to extract stress intensity factors. The $J$-integral is widely accepted as a fracture mechanics parameter for both linear and nonlinear material response. It is related to the energy release associated with crack growth and is a measure of the intensity of deformation at a notch or crack-tip. Under linearly elastic material assumptions, the $J$-integral, $J$, can be interpreted as being equivalent to the energy release rate, $G$. Because of the importance of the $J$-integral in the assessment of flaws, its accurate numerical evaluation is vital to the practical application of fracture mechanics in design calculations. The details of the $J$-integral method for stress intensity factor extraction from finite element analyses can be found in References [43] and [53].

\subsection{Specimens}

All of the finite element models of this study were 3-D models. Even though the through-crack model can be modeled in a 2-D finite element model, it was convenient to model it in a 3-D model because ZENCRACK provides crack-blocks in 3-D finite element models. The elements used were 20-noded C3D20 continuum elements. Detailed information about this element can be obtained from Reference [53], but essentially the C3D20 continuum element is a second-order element that provides higher accuracy than a first-order element for "smooth" problems that do not involve complex contact conditions, impact, or severe element distortions. It also captures stress concentrations more effectively and is better for modeling geometric features. It can be used to model a curved surface with an efficient number of elements. Finally, 
second-order elements are very effective in bending-dominated problems, because each node has three degrees of freedom.

All of the specimens were loaded in Mode I by uniformly distributed tractions applied in tension along the end surfaces perpendicular to the longest axis of the specimen. The applied load for all of the finite element models was $S_{\text {app }}=1.0 \mathrm{MPa}$. Symmetry of the specimen allowed for the modeling of only half of the specimen.

The ZENCRACK crack-block library contains two topological types of crack-blocks; quarter circular crack-blocks, and through crack-blocks. The naming convention of the crackblocks is based on these topological types. "sq" and "st" refer to quarter circular and through crack-blocks respectively. The first number in each name indicates the total number of elements in the crack-block, the second gives the number of elements along the crack front. A surface crack is modeled using two quarter circular crack-blocks. Figures 3.7 through 3.10 contain illustrations of examples of the finite element models for the various specimen types and crackblocks. Figure 3.7 contains a depiction of a finite model of the smooth unnotched specimen for a corner crack with a length and depth of $1.016 \mathrm{~mm}$. The crack-block type used was the sq112x4c. This crack-block uses four continuum elements along its crack front. Therefore there are nine nodes along the perimeter of the crack front. The ABAQUS output included nine stress intensity factors calculated at each of the crack front nodes. A sample of the $K_{\mathrm{t}}=3$ specimen model for a surface crack of length and depth of $0.254 \mathrm{~mm}$ is shown in Figure 3.8. The surface crack was modeled with two sq103x4 type crack-blocks. Both blocks contributed to seventeen crack front nodes and therefore seventeen stress intensity factor values were calculated across the crack front. Figure 3.9 contains the finite element model of a surface crack in the $K_{\mathrm{t}}=4$ specimen. The crack had a length and depth of $0.635 \mathrm{~mm}$ that was modeled with two sq112ax4 
types of crack-blocks. Seventeen stress intensity factors values were computed along the crack front of this model. Figure 3.10 contains an illustration of the finite element model of $K_{\mathrm{t}}=5$ specimen with a through crack of length $9.869 \mathrm{~mm}$. The through crack was modeled with st28x1 type crack-block. This crack-block uses one continuum element along its crack front hence three nodes along the perimeter of the crack front. ABAQUS was used to compute the three stress intensity factors at each of the crack front nodes 

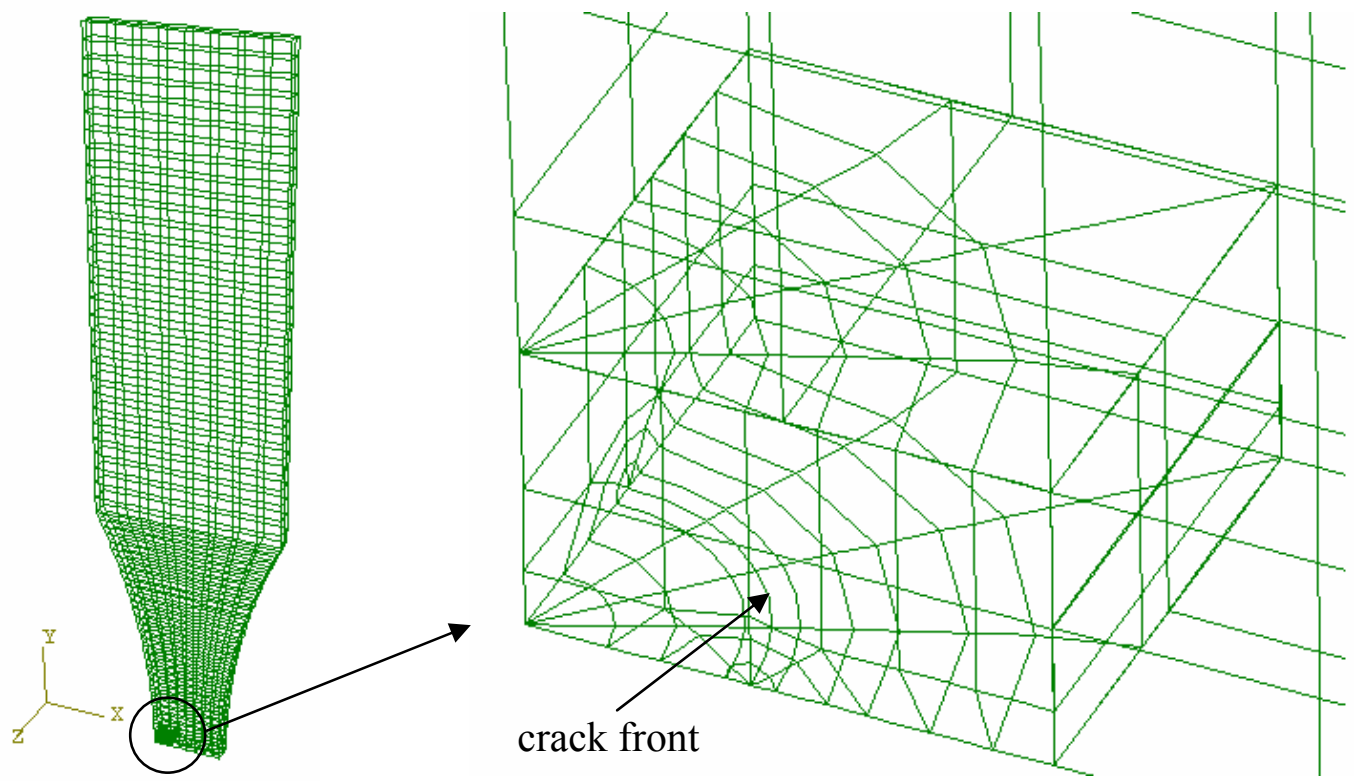

Figure 3.7 Finite element grid in the vicinity of the crack-tip of a smooth unnotched specimen with a corner crack $(a=c=1.016 \mathrm{~mm}$; sq112x4c crack-block; total number of elements $=1,112$; total number of nodes $=6,696$; total degrees of freedom $=20,088$ )

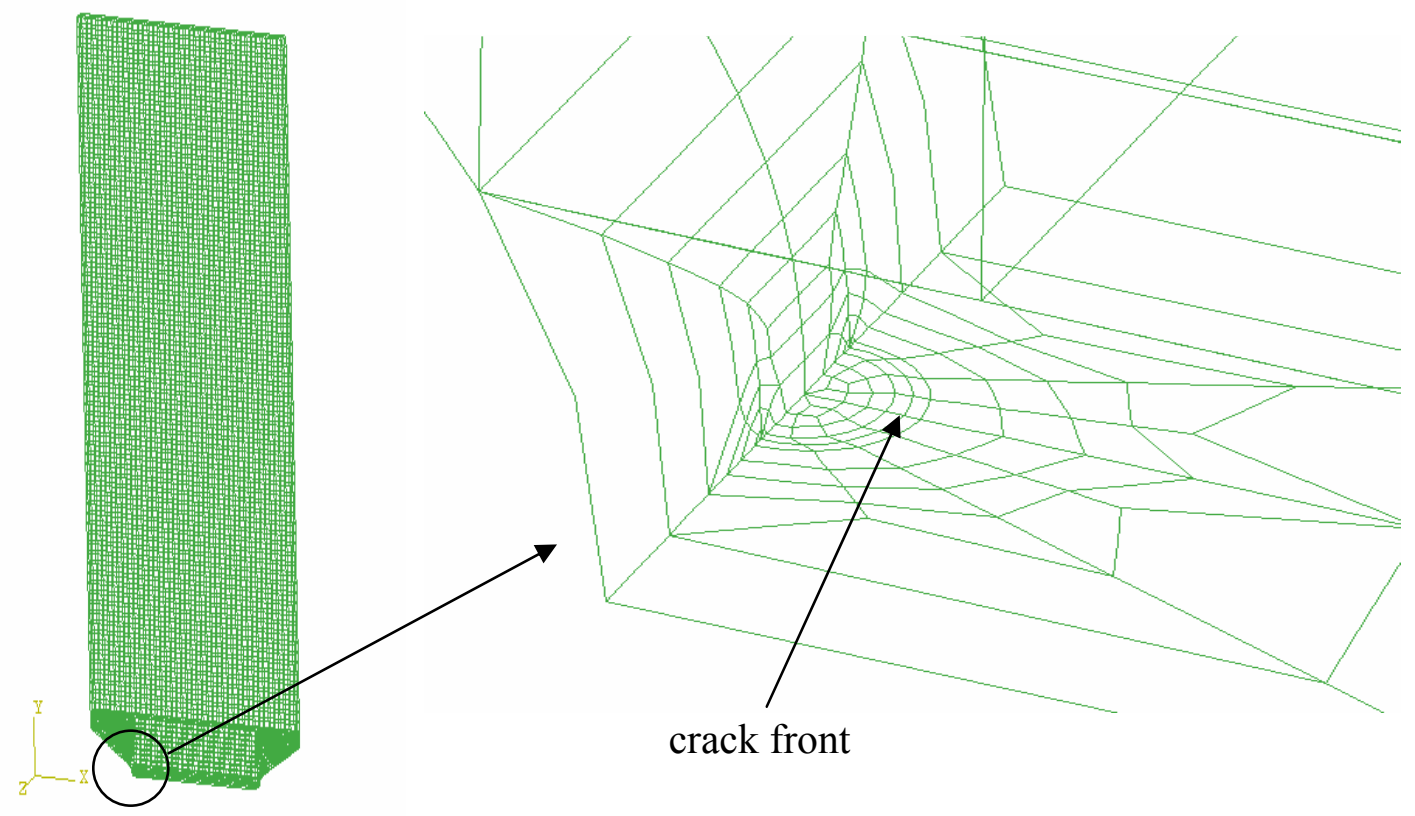

Figure 3.8 Finite element grid in the vicinity of the crack-tip of a $K_{\mathrm{t}}=3$ specimen with a surface crack ( $a=c=0.254 \mathrm{~mm}$; two sq103x4 crack-blocks; total number of elements $=7,222$; total number of nodes $=41,066$; total degrees of freedom $=123,198$ ) 

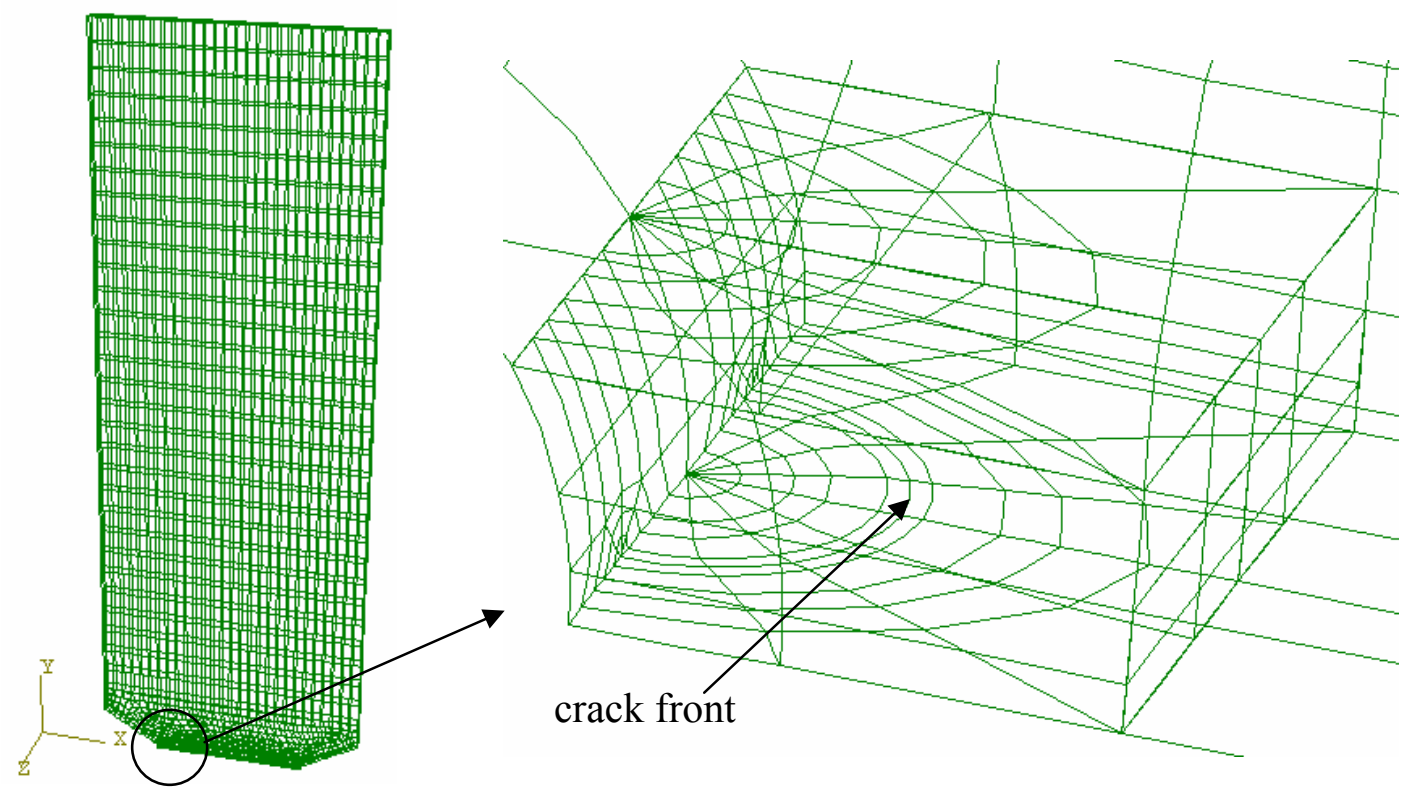

Figure 3.9 Finite element grid in the vicinity of the crack-tip of a $K_{\mathrm{t}}=4$ specimen with a surface crack ( $a=c=0.635 \mathrm{~mm}$; two sq112ax4 crack-blocks; total number of elements $=2,646$; total number of nodes $=15,085$; total degrees of freedom $=45,255$ )

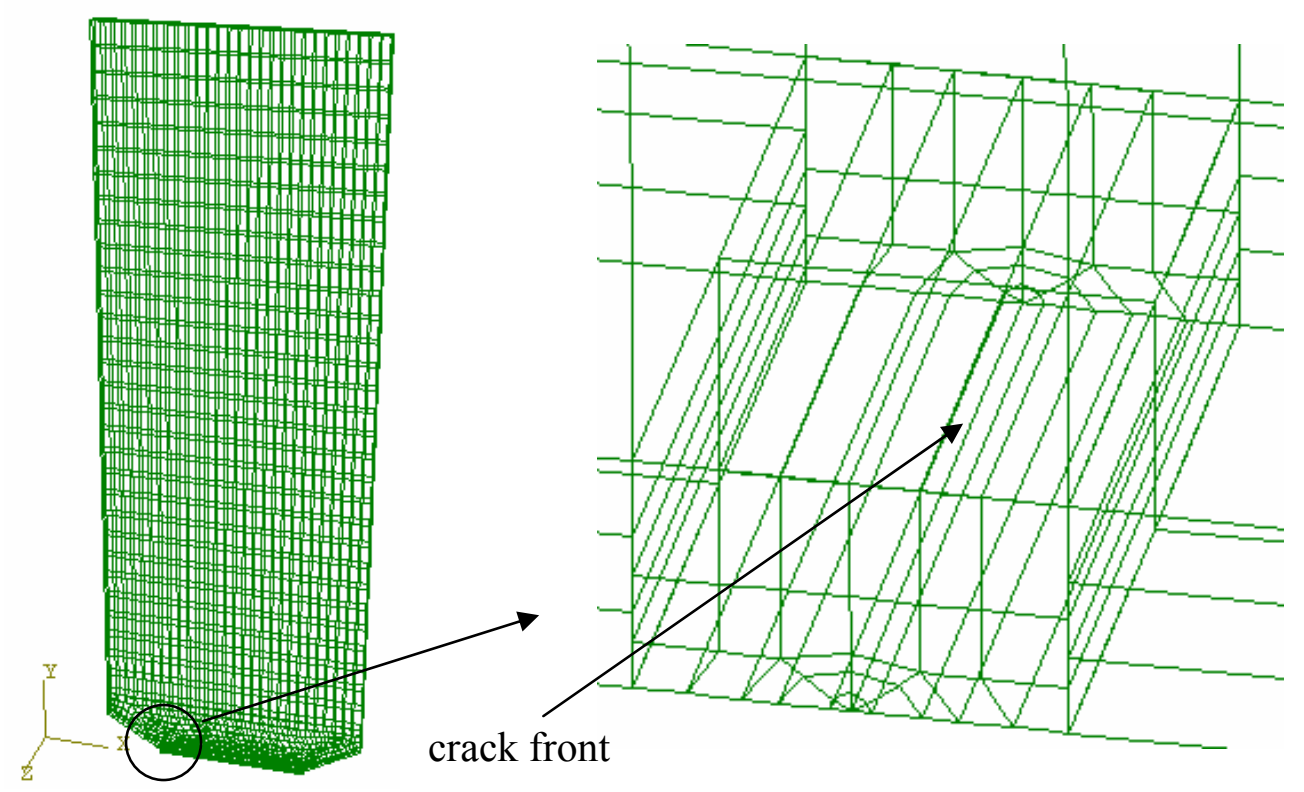

Figure 3.10 Finite element grid in the vicinity of the crack-tip of a $K_{t}=5$ specimen with a through-crack ( $c=9.869 \mathrm{~mm}$; st28x 1 type crack-block used; total number of elements $=2,486$; total number of nodes $=14,408$; total degrees of freedom $=43,224$ ) 


\section{CHAPTER 4}

\section{FASTRAN-II}

\subsection{Introduction}

The objective of this chapter is to provide an explanation of some of the important parameters associated with the FASTRAN-II input file for crack growth analysis. The version of FASTRAN-II used in this study was version 3.81. FASTRAN-II analyses were performed by executing the fastran381.exe executable file and then specifying names of both input and output file names. A typical FASTRAN-II input file is shown in Appendix A and Reference [2] is the FASTRAN-II manual.

\subsection{Mechanical and Fracture Properties}

One of the inputs to FASTRAN-II is the effective stress intensity factor range, $\Delta K_{\text {eff }}$ as a function of the crack growth rate, $d c / d N$. These data must be obtained for the material of interest over the widest range in rates possible from threshold (low growth rates) to fracture (large growth rates), especially if spectrum load predictions are required. Measured fatigue data for the configuration of interest would be helpful, but it is not essential. The use of plasticitycorrected stress intensity factors is only necessary if severe loading, such as low cycle fatigue conditions are of interest. Most crack life calculations can be performed using the linear elastic stress intensity factor analysis with crack closure modifications.

Under constant amplitude loading, another important input variable is the constraint factor, $\alpha_{\text {constraint }}$. As explained in section 1.6, the constraint factor is used to elevate the flow stress to $\alpha_{\text {constraint }} \sigma_{o}$ at the crack tip to account for the influence of a 3-D stress state. The flow 
stress, $\sigma_{0}$, can be defined to be the average of the yield stress, $\sigma_{y s}$, and the ultimate tensile strength, $\sigma_{u}$, of the material. For plane stress conditions, $\alpha_{\text {constraint }}$ was set equal to 1.0; and for simulated plane strain conditions, $\alpha_{\text {constraint }}$ was set equal to 3.0. The correct value of the constraint factor will correlate the constant amplitude fatigue crack growth rate data over a wide range of stress ratios [54]. According to Reference [2], for ideal full plane strain conditions, an $\alpha_{\text {constraint }}$ value of 3.0 is recommended, while for full plane stress conditions an $\alpha_{\text {constraint }}$ value of 1.0 is recommended. When analyzing crack growth rate data from a specimen with a through crack, such as a compact tension or centre crack tension specimen, Newman et al. [1] recommends a procedure whereby a high $\alpha_{\text {constraint }}$ (plane strain) is assumed for low crack growth rates where the linear elastic stress intensity factor range, $\Delta K$ is low. A low $\alpha_{\text {constraint }}$ is used at high crack growth rates in the constraint loss regime where $\Delta K$ is high. The actual values of constraint factor were chosen to best collapse the crack growth rate versus $\Delta K$ curves for various stress ratios into one crack growth rate versus effective stress intensity factor range, $\Delta K_{\text {eff }}$, curve.

The mechanical, fracture and crack growth properties for the 2024-T3 aluminum alloy used in this study were obtained from Reference [1] and summarized in Table 4.1. The $0.2 \%$ offset yield stress and ultimate tensile strength are designated by $\sigma_{y s}$ and $\sigma_{u}$ respectively. Young's modulus of elasticity is designated by $E$ while $K_{\mathrm{F}}$ and $m$ is the elastic-plastic the fracture toughness and fracture toughness parameter of the Two-Parameter Fracture Criterion. The Two-Parameter Fracture Criterion is a fracture criterion that accounts for the elastic-plastic behavior of the material. The $m$ parameter is usually between 0.0 and 1.0. For linear elastic fracture mechanics analysis it recommended to use an $m$ value of 0.0 for brittle materials and an 
$m$ value of 1.0 is recommended for very ductile materials. Details of this fracture criterion can be found in Reference [2]. Table 4.1 also contains tabulated values of effective stress intensity factor range, $\Delta K_{\text {eff }}$, versus crack growth rate, and constraint factor, $\alpha_{\text {constraint }}$, versus crack growth rate.

\section{Table 4.1}

Mechanical, fracture and baseline crack-growth ( $\Delta K_{\text {eff }}$-rate) properties for 2024-T3 aluminum (from Reference [1])

(Units for $\Delta K_{\text {eff }}$ is $M P a \sqrt{m}$ and $d c / d N$ is $m / c y c l e$ )

\begin{tabular}{|c|c|c|c|}
\hline \multicolumn{2}{|c|}{$\sigma_{\mathrm{ys}}=360 \mathrm{MPa}$} & & \\
\hline \multicolumn{2}{|c|}{$\sigma_{\mathrm{u}}=490 \mathrm{MPa}$} & & \\
\hline \multicolumn{2}{|c|}{$E=72000 \mathrm{MPa}$} & & \\
\hline \multicolumn{2}{|c|}{$K_{\mathrm{F}}=267 M P a \sqrt{m}$} & & \\
\hline \multicolumn{2}{|c|}{$m=1.0$} & & \\
\hline$\Delta K_{\text {eff }}$ & $d c / d N$ & $\alpha_{\text {constraint }}$ & $d c / d N$ \\
\hline 0.80 & $1.0 \mathrm{e}-12$ & 2.00 & $1.0 \mathrm{e}-07$ \\
\hline 1.10 & $5.0 \mathrm{e}-11$ & 1.00 & $2.5 \mathrm{e}-06$ \\
\hline 2.05 & $2.0 \mathrm{e}-09$ & & \\
\hline 4.00 & $8.0 \mathrm{e}-09$ & & \\
\hline 7.70 & $1.0 \mathrm{e}-07$ & & \\
\hline 13.50 & $1.0 \mathrm{e}-06$ & & \\
\hline 23.00 & $1.0 \mathrm{e}-05$ & & \\
\hline 36.00 & $1.0 \mathrm{e}-04$ & & \\
\hline 85.00 & $1.0 \mathrm{e}-02$ & & \\
\hline
\end{tabular}




\subsection{Stress Intensity Factor}

As previously mentioned in Chapter 3, both the smooth unnotched specimen and the three double-edge V-notched specimen types were not members of the predefined crack menu of FASTRAN-II. This user specified configurations require stress intensity factors at various crack lengths to be included in the input file. The averaged stress intensity factors obtained from finite element analyses discussed in Chapter 3 were used for this purpose. The input format required two columns of values of the ordered pairs of nondimenzionalized crack length and the corresponding boundary correction factor, $F(c)$, with the nondimenzionalized crack length ranked in ascending order. The boundary correction factor was derived from the averaged stress intensity factor and is a function of load, geometric configuration, and flaw size. The derivation details for each specimen are presented in the coming sections. Up to 50 pairs of values can be provided as input in the FASTRAN-II code. It should be noted that for the user input configuration, FASTRAN-II does not have the capability of calculating crack propagation in the depth direction; only in the width direction. For a part-though flaw the averaged stress intensity factor was used in the analysis, instead of the stress intensity factor that corresponded to the width direction. In this study it was assumed that a crack forms as a single initial part-through corner flaw or as a single part-through surface flaw. Two separate input files were prepared; one associated with the corner crack assumption and the other associated with the surface crack assumption. 


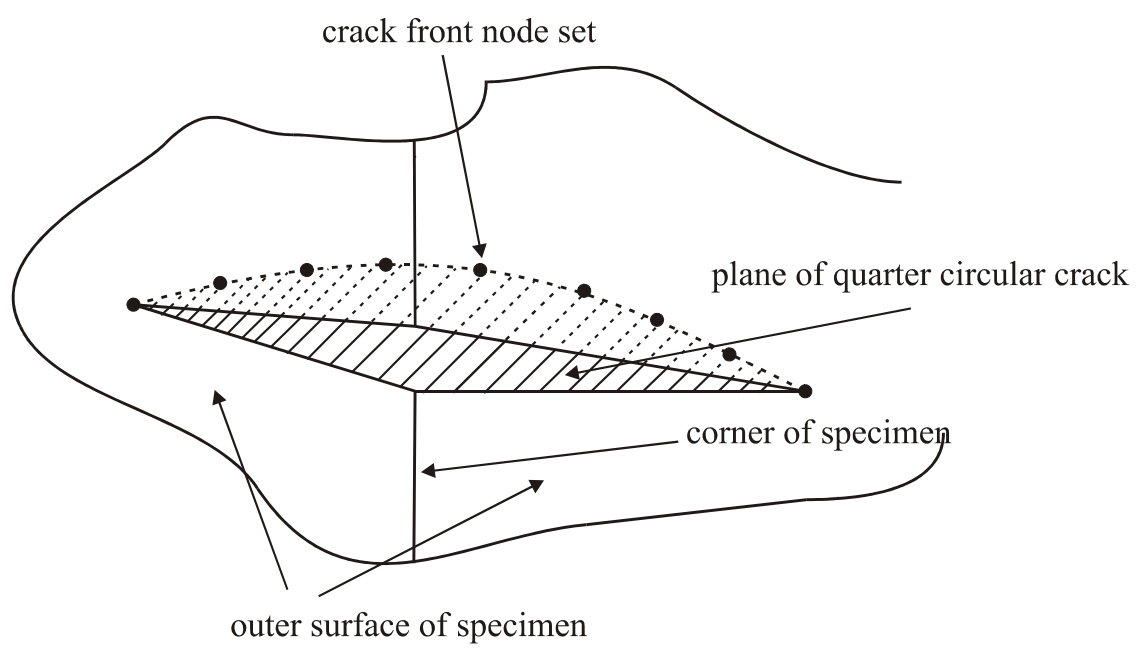

Figure 4.1 Crack front node set for the quarter circular corner crack

\subsection{Smooth Unnotched Specimen}

The FASTRAN-II input file required normalized crack lengths, $c / w_{\mathrm{n}}$, and boundary correction factors, $F(c)$, supplied in a tabular format for smooth unnotched specimen. The boundary correction factor, $F(c)$, were derived from the stress intensity factor using the following defining equation:

$$
F(c) \equiv \frac{K_{\mathrm{I}}}{S_{\mathrm{app}} \sqrt{\pi c}}
$$

where $c$ is the crack length in the width direction, $w_{\mathrm{n}}$ is the width of the specimen in the plane of the crack, $S_{\text {app }}$ is the applied remote stress and $K_{\mathrm{I}}$ is the averaged mode $\mathrm{I}$ stress intensity factor associated with the stress field at the crack tip obtained from finite element analysis. Figure 4.2 illustrates some of the nomenclature used for the smooth unnotched specimen. 

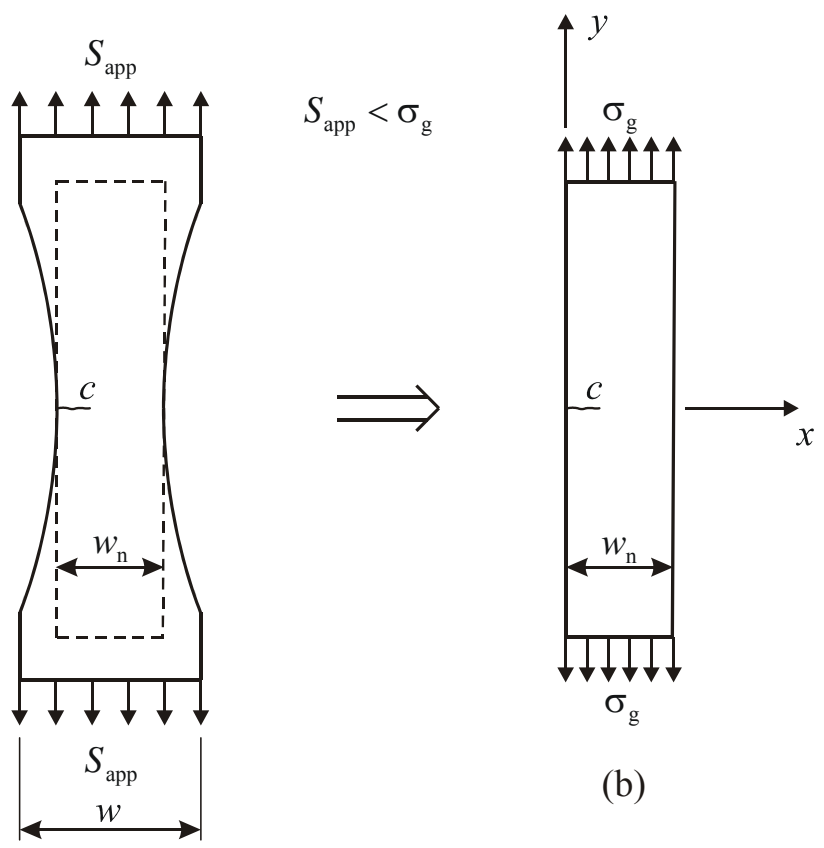

(a)

Figure 4.2 Nomenclature for smooth unnotched specimen

Due to the hourglass shape of the smooth unnotched specimen it can be treated as a finite width rectangular plate with width $w_{\mathrm{n}}$ and applied stress $\sigma_{\mathrm{g}}$ as is depicted in Figure 4.1(b). From equilibrium of forces, the remote stress of the idealized finite width plate, $\sigma_{g}$, can be expressed as

$$
\sigma_{\mathrm{g}}=\frac{S_{\mathrm{app}} w}{w_{\mathrm{n}}}
$$

For the specimen in Figure 4.1(b),

$$
F(c)=\frac{K_{\mathrm{I}}}{\sigma_{\mathrm{g}} \sqrt{\pi c}}
$$

When the crack length in the direction of the width of the specimen is very small, $c \rightarrow 0$, the geometric correction factor, $F(c)$, was determined with the aid of the appropriate stress 
concentration factor. During crack growth analysis with FASTRAN-II the $F(c)$ values of the tabular input are converted to $K_{\mathrm{I}}$ values using equation (4.3) for a particular crack length. The incremental crack length is computed and added to the current crack length. This procedure was done iteratively until a specified criterion was met. For intermediate crack lengths, which do not fall into the tabular input values FASTRAN-II interpolates between two existing values.

One problem did occur when calculating the equivalent initial flaw sizes. Since equivalent initial flaw sizes fall within the range of $10^{-5}$ and $10^{-6} \mathrm{~mm}$, it was necessary to determine stress intensity factors at these very small crack lengths. But, it was not feasible to create a finite element mesh for such small crack lengths. In fact, the smallest crack length that was modeled for the smooth unnotched specimen was a $c=0.019 \mathrm{~mm}$. The limiting case solution concept was used to determine a value of $F(c)$ when the crack length $c$ approached zero. Consequently, crack growth analysis could be executed for crack lengths that fall between $c$ was near zero and $c=0.019 \mathrm{~mm}$.

This approach can be summarized in the following manner. Consider the stresses acting at the vicinity of crack tip of a very small crack, that is as $c \rightarrow 0$, as illustrated in Figure 4.3. The localized normal stress, $\sigma_{y y}$, at the crack tip, due to stress concentration, can be written as

$$
\sigma_{y y}=K_{\mathrm{tg}} \sigma_{\mathrm{g}}
$$

where $K_{\mathrm{tg}}$ is the gross stress factor and $\sigma_{\mathrm{g}}$ is the remote stress (see Figure 4.2(b)). The stress concentration factor, $K_{\text {tg }}$, equals 1.0 for a rectangular specimen, consequently

$$
\sigma_{y y}=\sigma_{\mathrm{g}}
$$

The stress intensity factor for a semi-infinite panel with an edge crack is given by,

$$
K_{\mathrm{I}}=1.122 \sigma \sqrt{\pi c}
$$


Substituting equation (4.5) into equation (4.6),

$$
K_{\mathrm{I}}=1.122 \sigma_{\mathrm{g}} \sqrt{\pi c}
$$

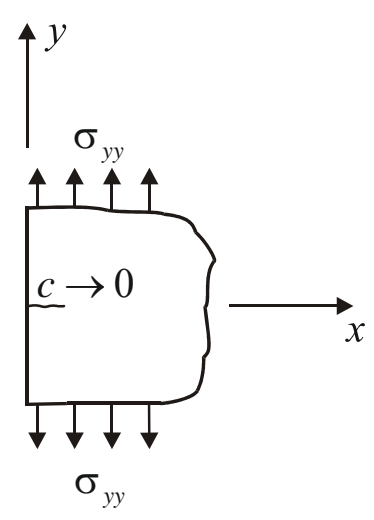

Figure 4.3 Local stresses acting in the vicinity of a crack tip as the crack length, $c$, approaches zero

Substituting equation (4.7) into equation (4.3), yields the following

$$
\lim _{c \rightarrow 0} F(c)=\frac{K_{\mathrm{I}}}{\sigma_{\mathrm{g}} \sqrt{\pi c}}=1.122
$$

The normalized crack length of 0.0 and limiting value of 1.122 was the first input pair of values in the tabular format of nondimenzionalized crack lengths and the corresponding boundary correction factors

Up to 50 pairs of nondimensionalized crack length and the corresponding boundary correction factor could be inputted into the FASTRAN-II code. The more values provided, the better the accuracy of the life prediction. A finite element model was required for every crack size. From Table 3.1, for the smooth unnotched case, nine stress intensity factors were computed for corner crack cases, nine for surface crack cases, and six for the through crack cases. Rather than constructing 50 finite element models to calculate 50 stress intensity solutions a cubic interpolation technique was utilized to obtain intermediate values. That is, a series of unique 
cubic polynomials were fitted between the data points, with the stipulation that the curve be continuous and smooth. These cubic splines were then used to interpolate data points between sampled data points generated with the finite element code. Cubic splines are popular because they are easy to implement and produce a seamless curve. The cubic polynomials were fitted to the $c$ versus $K_{\mathrm{I}}$ data, because $c$ is a predictable exponential functional of $K_{\mathrm{I}}$. Once the 50 ordered pairs of $c$ and $K_{\mathrm{I}}$ values were obtained by cubic polynomial fitting, the data was converted to nondimensionalized crack length and the corresponding boundary correction factor values.

Figure 4.4 contains the plot of stress intensity factor, $K_{\mathrm{I}}$ as a function of the crack length, $c$, for the case of the smooth unnotched specimen with a quarter circular corner crack assumption. The thickness of the smooth unnotched specimen was $2.286 \mathrm{~mm}$, therefore for each crack length that was less than $2.286 \mathrm{~mm}$, the stress intensity factor value was averaged from the values obtained at crack front nodes. This practice was implemented following the advice of Dr. Jim Newman through personal communication. The stress intensity factor values for crack length above $2.286 \mathrm{~mm}$ were through crack values.

Figure 4.5 contains a similar plot of the boundary correction factor for the smooth unnotched specimen with a surface crack. It can be seen from the plots that two additional data points were determined between the data points obtained from finite element analysis using the cubic splines. Also note that at very small crack lengths the data points are sparse. This is due to the inability of the ZENCRACK code to create a very fine mesh for very small crack lengths $(c<0.019 \mathrm{~mm})$. The $c$ and $K_{\mathrm{I}}$ values were converted to $c / w_{\mathrm{n}}$ and $F(c)$ respectively and used in the input files. 


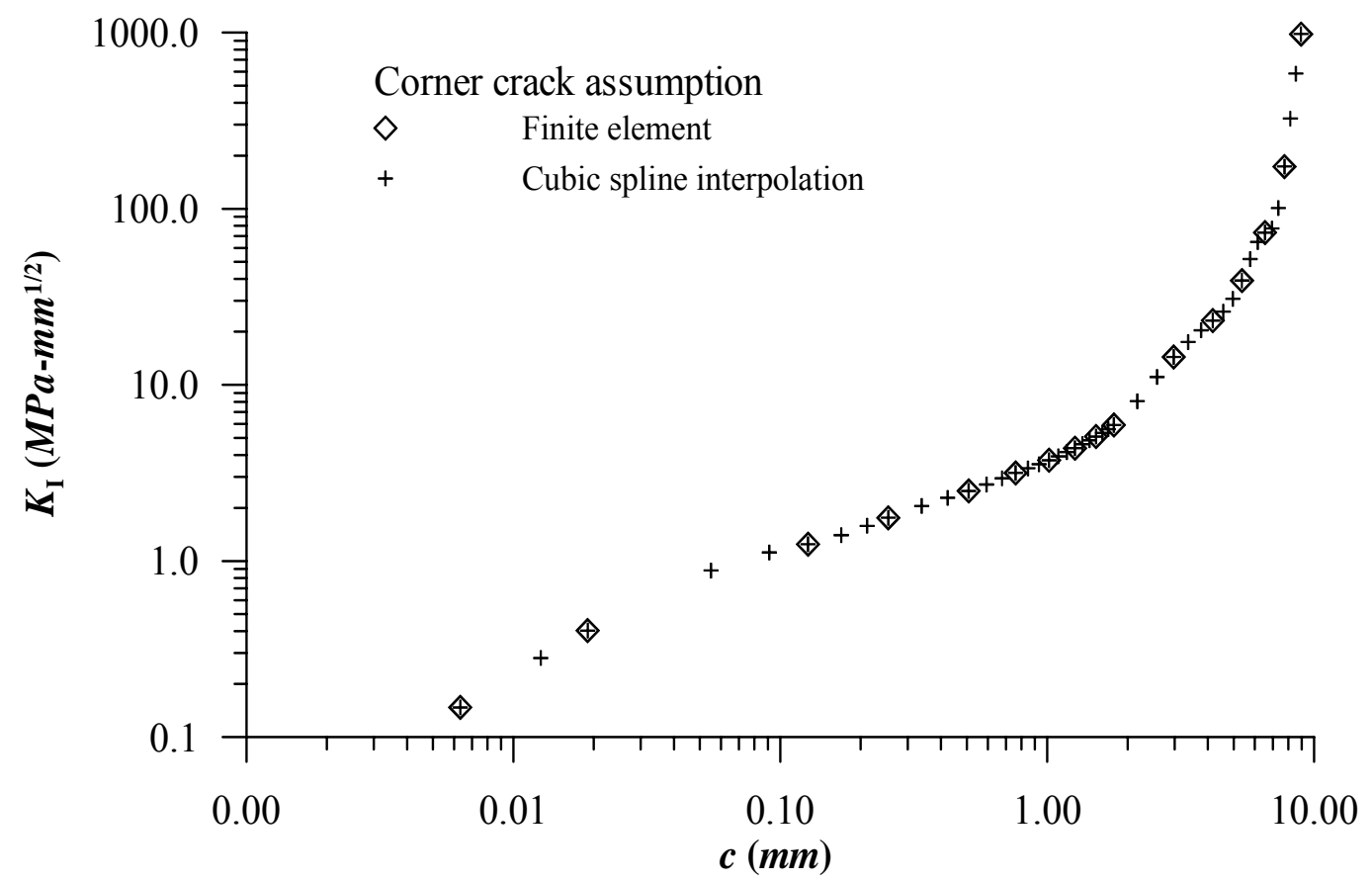

Figure 4.4 $K_{\mathrm{I}}$ vs. $c$ for smooth unnotched specimen with an initial corner crack

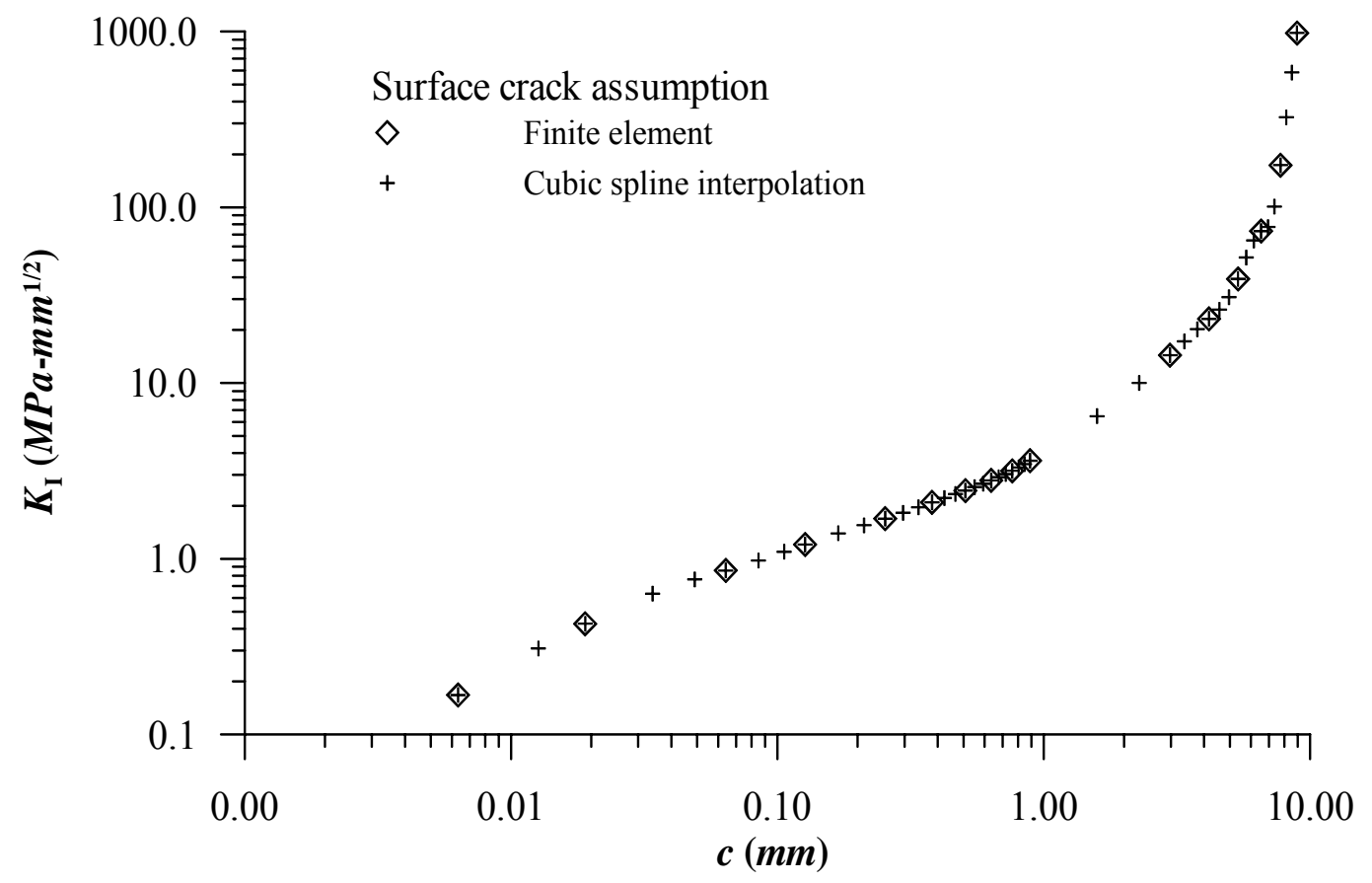

Figure 4.5 $K_{\mathrm{I}}$ vs. $c$ for smooth unnotched specimen with an initial surface crack 


\subsection{Double-Edge V-Notched Specimens}

Figure 4.6 depicts the nomenclature used for the $K_{t}=3,4$ and 5 double-edge V-notched specimens. Unlike the smooth unnotched specimen, V-notched specimen types have a constant cross-section except in the region of the notch. The boundary correction factor was obtained from equation (4.1) and the nondimenzionalized crack length was defined as $c / w$. Table 3.1 specifies the number of finite element models analyzed for each specimen type and flaw type.

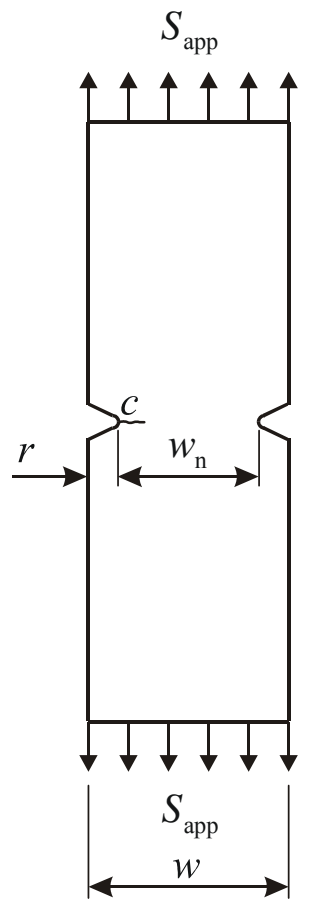

Figure 4.6 Nomenclature used for double-edge V-notched specimens

Just as for the case of the smooth unnotched specimen, the limiting case solution was derived for V-notched specimens. In the case of the double-edge V-notched specimens, the $K_{\mathrm{tg}}$ was obtained through the finite element analysis explained earlier in Chapter 2. The finite 
element analysis generated the maximum axial stress acting at the edge of the notch, $\sigma_{y y}$ max , and the gross stress concentration, $K_{\mathrm{tg}}$ was computed as

$$
K_{\mathrm{tg}}=\frac{\sigma_{y y_{\text {max }}}}{S_{\text {app }}}
$$

By using the nomenclature of Figures 4.3 and 4.6, the localized normal stress, $\sigma_{y y}$ is given as

$$
\sigma_{y y}=K_{\mathrm{tg}} S_{\mathrm{app}}
$$

Substituting equation (4.10) into equation (4.6) for a semi-infinite panel with edge crack,

$$
K_{\mathrm{I}}=1.122 K_{\mathrm{tg}} S_{\mathrm{app}} \sqrt{\pi c}
$$

Substituting, equation (4.11) into equation (4.1) yields the following:

$$
\lim _{c \rightarrow 0} F(c)=\frac{K_{\mathrm{I}}}{S_{\mathrm{app}} \sqrt{\pi c}}=1.122 K_{\mathrm{tg}}
$$

Therefore, this technique was used to determine the geometric correction factor for the case of a very small crack; that is the limit as the crack length approached zero. The limiting values for the $\mathrm{V}$-notched specimens are presented in Table 4.2.

Table 4.2

Limit case values for the double-edge $\mathrm{V}$-notched specimens (Units for $S_{\text {app }}$ and $\sigma_{y y_{\max }}$ are $M P a$ )

\begin{tabular}{|c|c|c|c|c|}
\hline$K_{\mathrm{t}}$ & $S_{\text {app }}$ & $\sigma_{y y_{\max }}$ & $K_{\mathrm{tg}}$ & $\underset{c \rightarrow 0}{\lim F(c)}$ \\
\hline 3 & 1.0 & 5.165 & 5.165 & 5.7951 \\
\hline 4 & 1.0 & 7.013 & 7.013 & 7.8686 \\
\hline 5 & 1.0 & 7.671 & 7.671 & 8.6069 \\
\hline
\end{tabular}

Figures 4.7 through 4.12 contain plots of stress intensity factor, $K_{\mathrm{I}}$, versus crack length, $c$, for the $\mathrm{V}$-notched specimens for both types of initial crack configurations. Both the finite 
element solution and the cubic spline interpolation are presented. Generally, the cubic spline was used to determine two additional data points between the finite element solutions. Some cubic spline data points were deliberately omitted because the total number of points was limited to 50 pairs for the FASTRAN-II code. Similar to the smooth unnotched specimen, the Vnotched specimens had the same thickness of $2.286 \mathrm{~mm}$. Therefore for crack lengths that were less than $2.286 \mathrm{~mm}$, the stress intensity factor value was averaged from the values obtained at the multiple crack front nodes. The $c$ and $K_{\mathrm{I}}$ values were converted to $c / w$ and $F(c)$ respectively and used in the input files. 


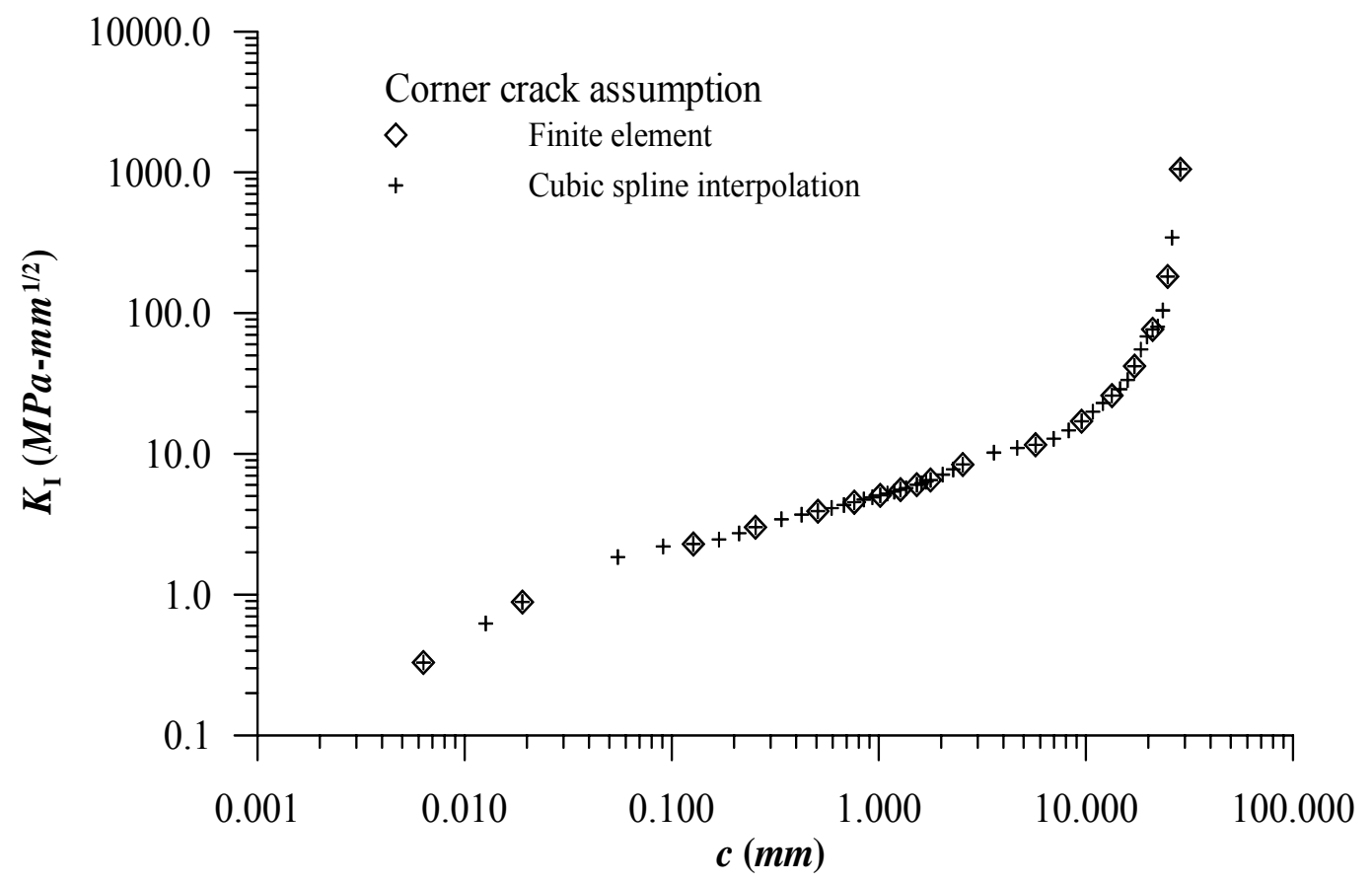

Figure 4.7 $K_{\mathrm{I}}$ vs. $c$ for $K_{\mathrm{t}}=3$ specimen with an initial corner crack

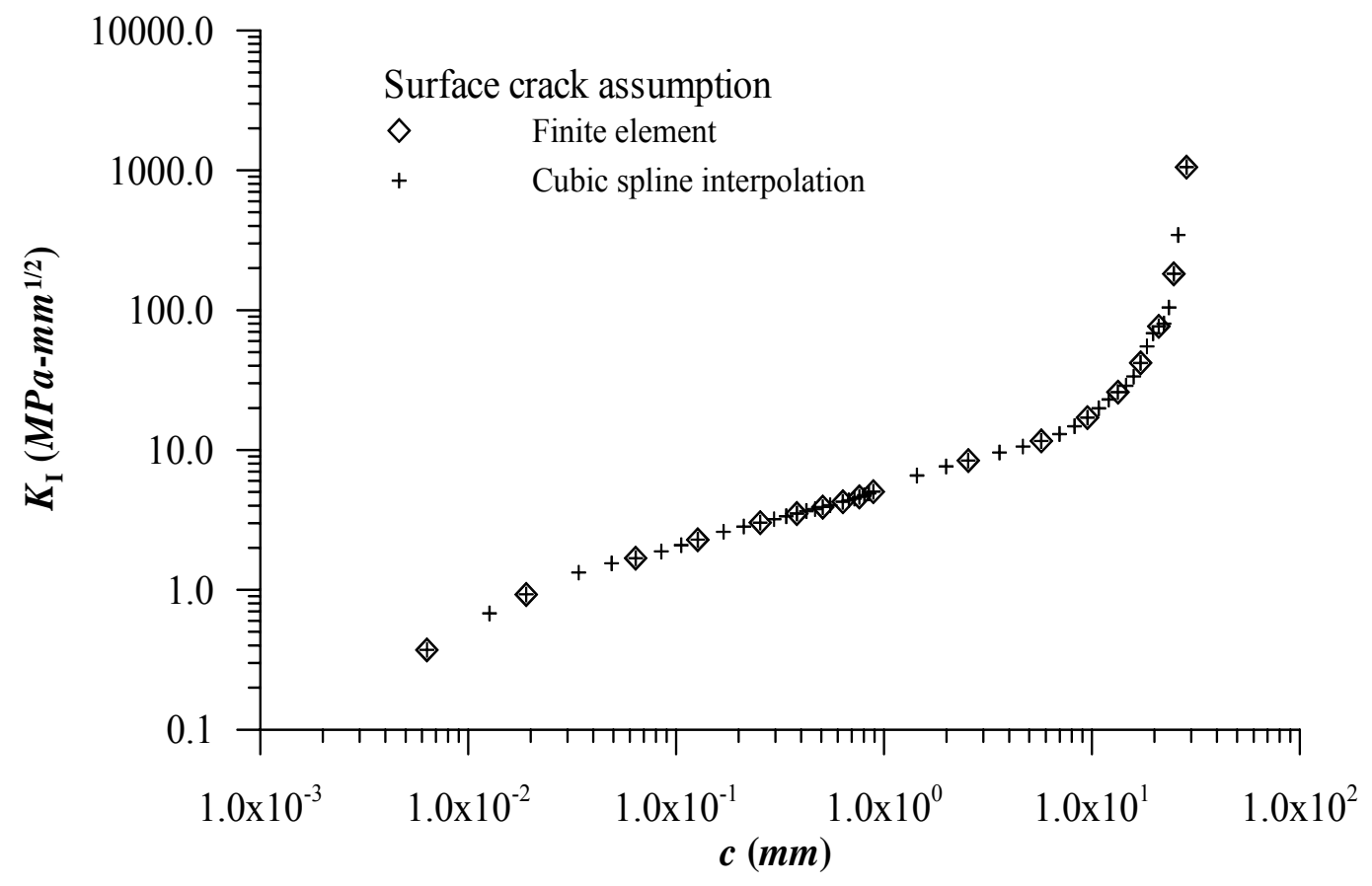

Figure 4.8 $K_{\mathrm{I}}$ vs. $c$ for $K_{\mathrm{t}}=3$ specimen with an initial surface crack 


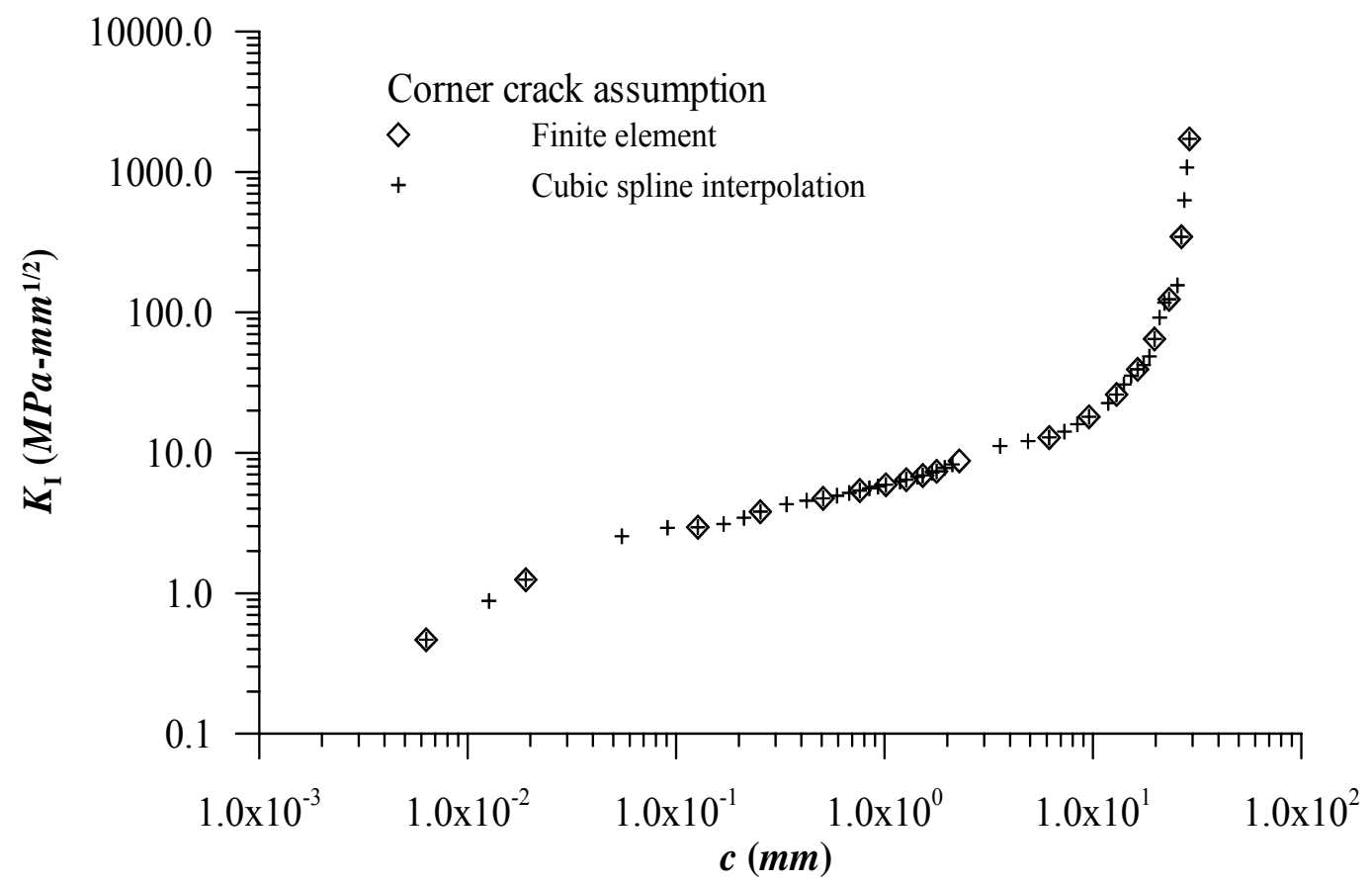

Figure 4.9 $K_{\mathrm{I}}$ vs. $c$ for $K_{\mathrm{t}}=4$ specimen with an initial corner crack

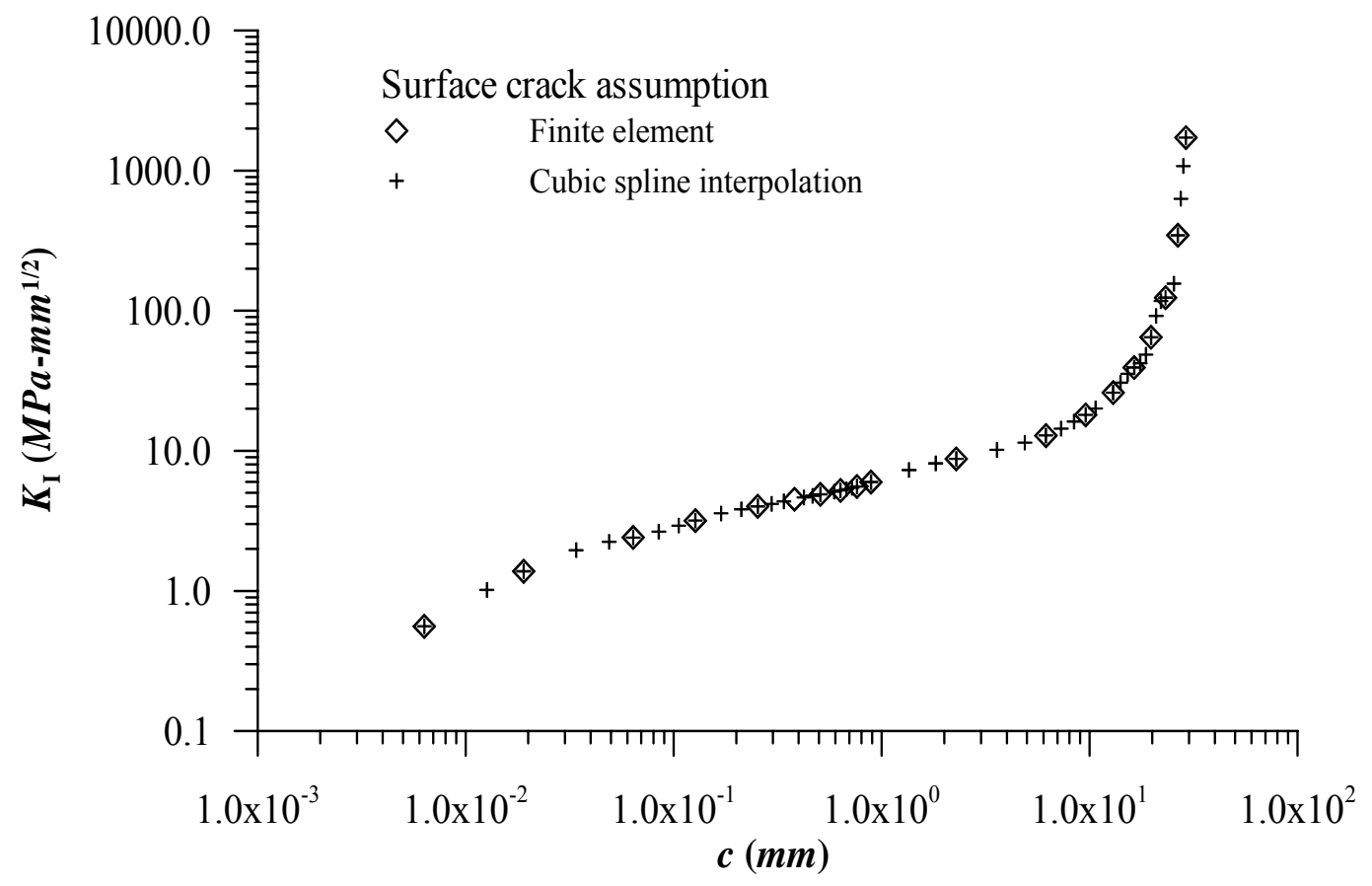

Figure 4.10 $K_{\mathrm{I}}$ vs. $c$ for $K_{\mathrm{t}}=4$ specimen with an initial surface crack 


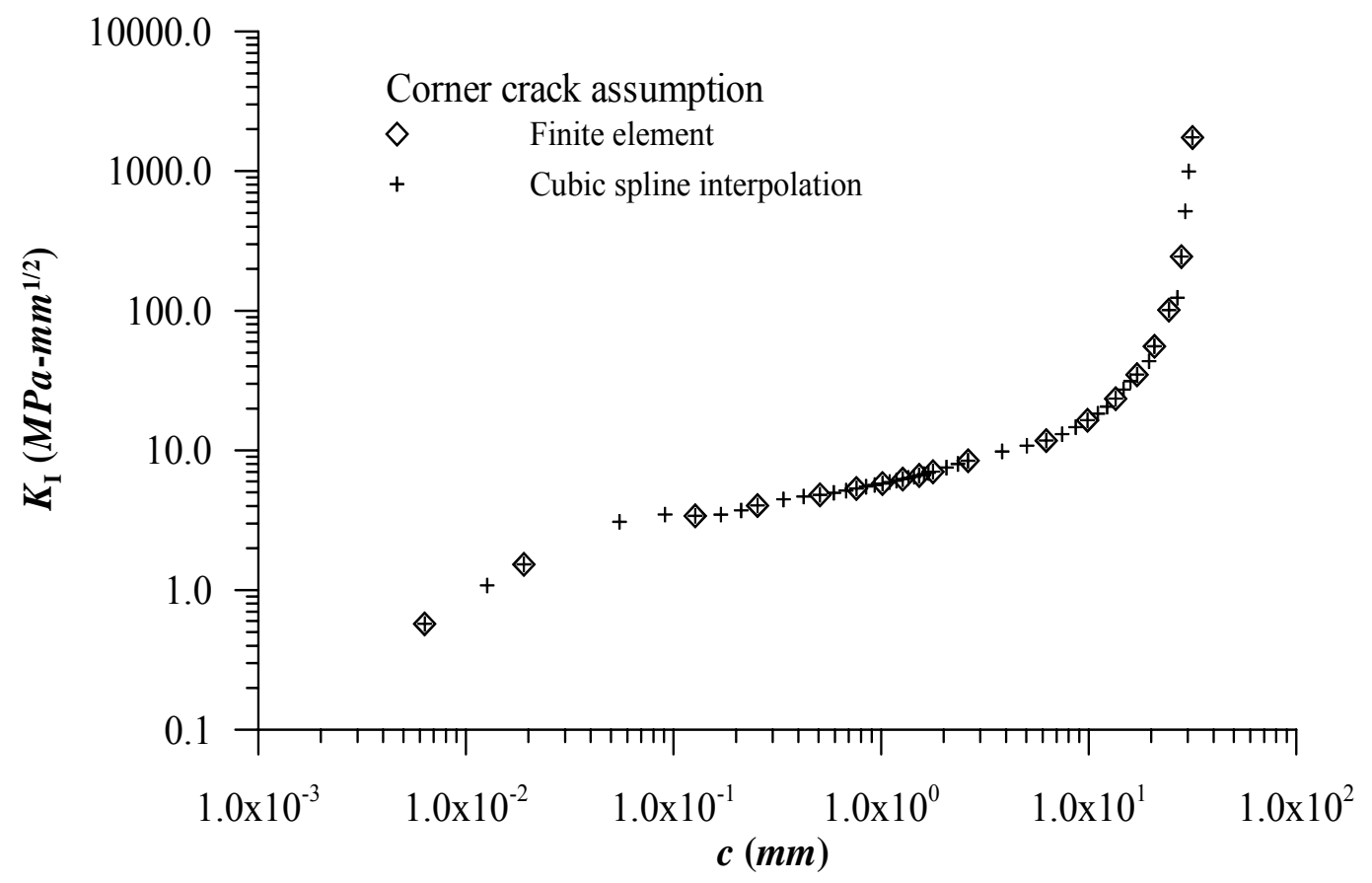

Figure 4.11 $K_{\mathrm{I}}$ vs. $c$ for $K_{\mathrm{t}}=5$ specimen with an initial corner crack

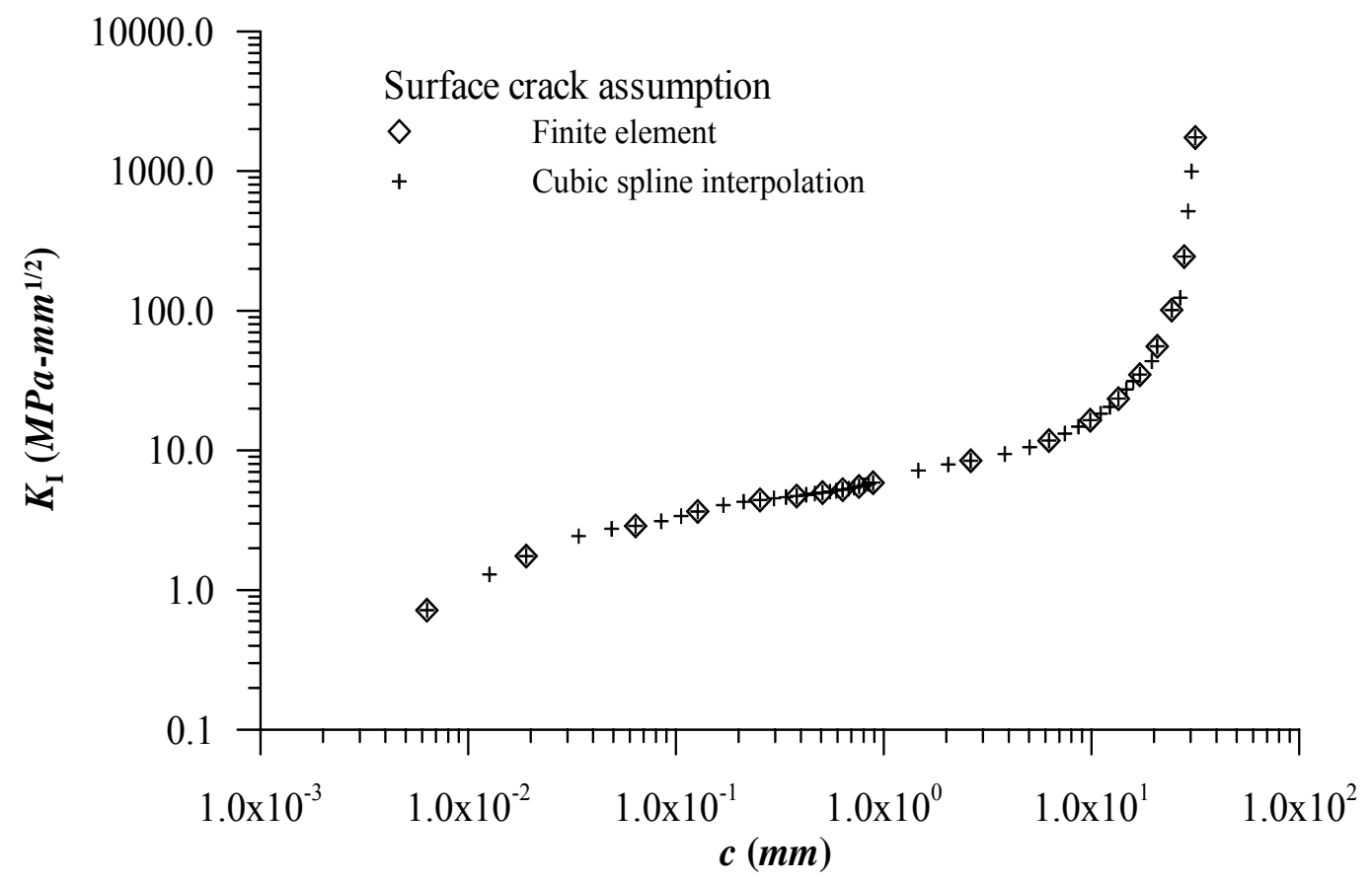

Figure 4.12 $K_{\mathrm{I}}$ vs. $c$ for $K_{\mathrm{t}}=5$ specimen with an initial surface crack 


\section{CHAPTER 5}

\section{STATISTICAL ANALYSES AND RESULTS}

\subsection{Statistical Relationships}

During the course of this study an attempt was made to determine the statistical relation between the equivalent initial flaw size distribution and the combination of the geometric configuration and the loading condition. The following four factors were identified as the principal parameters to define the geometry and load. Two factors for the geometric configuration - stress concentration factor, $K_{\mathrm{tg}}$, and uncracked ligament length, $w_{\mathrm{n}}$, and two factors defining the constant amplitude cyclic stress conditions-maximum stress, $S_{\max }$, and mean stress, $S_{\text {mean }}$.

The following approach was taken to develop the statistical relationship. First, equivalent initial flaw sizes were determined for the smooth unnotched specimens, the open-hole specimens, and the $K_{\mathrm{t}}=5$ specimens by using FASTRAN-II crack growth code. The statistical relation was then determined for the equivalent initial flaw size distribution parameter as function of geometric and load factors for these specimens by using multiple regression analyses. The objective of this phase of the study was not to predict a specific value of equivalent initial flaw size, $c_{0}$, but rather an estimated distribution of $c_{0}$. This distribution of $c_{0}$ could then be used as an input into the FASTRAN-II code to generate a distribution of estimated fatigue life for the specimen. This form of simulation is called the Monte Carlo simulation.

In this study the distribution of equivalent initial flaw size, $c_{0}$, was modeled as a normal distribution. Therefore, by regressing the distribution parameters, such as mean and standard deviation, on independent variables, $K_{\mathrm{tg}}, w_{\mathrm{n}}, S_{\text {mean }}$, and $S_{\max }$, an estimation equation for each 
mean and standard deviation as functions of these independent variables was obtained. The mean and standard deviation values were then obtained from these estimation equations by substituting values of the independent variables for the $K_{t}=3$ and $K_{t}=4$ specimens. Using the mean and standard deviation values, random equivalent initial flaw sizes were then generated from a normal distribution. The random distribution of the equivalent initial flaw size was then used an input into FASTRAN-II to generate estimated fatigue lives for the $K_{t}=3$ and $K_{t}=4$ specimens. Estimated fatigue lives were computed for both the assumed corner and surface initial flaw configurations.

\subsection{Calculating Equivalent Initial Flaw Size}

For each point on an experimentally generated $S-N$ curve there exists an equivalent initial flaw size. The following numerical procedure was employed to determine the equivalent initial flaw size. First, two initial flaw sizes $\left(c_{\mathrm{U}}\right.$ and $\left.c_{\mathrm{L}}\right)$ were identified by trial and error using the FASTRAN-II code. The predicted fatigue life associated with $c_{\mathrm{U}}, N_{\mathrm{FAST}}\left(c_{\mathrm{U}}\right)$, would be greater than the predicted fatigue life, $N$, associated with the actual point on the $S-N$ curve, and the predicted fatigue life associated with $c_{\mathrm{L}}, N_{\text {FAST }}\left(c_{\mathrm{L}}\right)$ was less than $N$.

$$
\begin{aligned}
& N_{\text {FAST }}\left(c_{\mathrm{L}}\right)<N \\
& N_{\text {FAST }}\left(c_{\mathrm{U}}\right)>N
\end{aligned}
$$

Using a simple linear interpolation method an approximate flaw size, $c_{\text {approx }}$ was calculated using

$$
c_{\text {approx }}=\frac{\left(c_{\mathrm{U}}-c_{\mathrm{L}}\right)\left[N-N_{\mathrm{FAST}}\left(c_{\mathrm{L}}\right)\right]}{N_{\mathrm{FAST}}\left(c_{\mathrm{U}}\right)-N_{\mathrm{FAST}}\left(c_{\mathrm{L}}\right)}+c_{\mathrm{L}}
$$


Equation (5.2) was derived by interpolating $c_{\text {approx }}$ between data points $\left[c_{\mathrm{L}}, N_{\mathrm{FAST}}\left(c_{\mathrm{L}}\right)\right]$ and $\left[c_{\mathrm{U}}, N_{\mathrm{FAST}}\left(c_{\mathrm{U}}\right)\right]$. A simple FORTRAN 90 interpolation code was written that used the FASTRAN-II code to compute $N_{\text {FAST }}\left(c_{\text {approx }}\right)$, predicted fatigue life, for a given initial flaw size. The interpolation code updated the initial flaw size to $C_{\text {approx }}$ in the FASTRAN-II input file and automatically executed FASTRAN-II. Once the execution was complete the predicted fatigue life, $N_{\text {FAST }}\left(c_{\text {approx }}\right)$ was obtained from the FASTRAN-II output file. $N_{\text {FAST }}\left(c_{\text {approx }}\right)$ was then compared to $N$ value. If $N_{\text {FAST }}\left(c_{\text {approx }}\right)$ met the convergence criteria, then $C_{\text {approx }}$ was the equivalent initial size; otherwise the procedure was iterated until $N_{\mathrm{FAST}}\left(c_{\text {approx }}\right)$ met the required convergence criteria. Figure 5.1 illustrates the iterative procedure involved in the calculation of initial flaw size. For each $\left(N, S_{\max }\right)$ pair an equivalent initial flaw size was calculated for the assumed corner crack configuration and another equivalent flaw size was calculated for the assumed surface crack configuration. Appendix B contains a listing of the FORTRAN 90 program used for calculating the equivalent initial flaw size for the $K_{\mathrm{t}}=5$ specimen. 


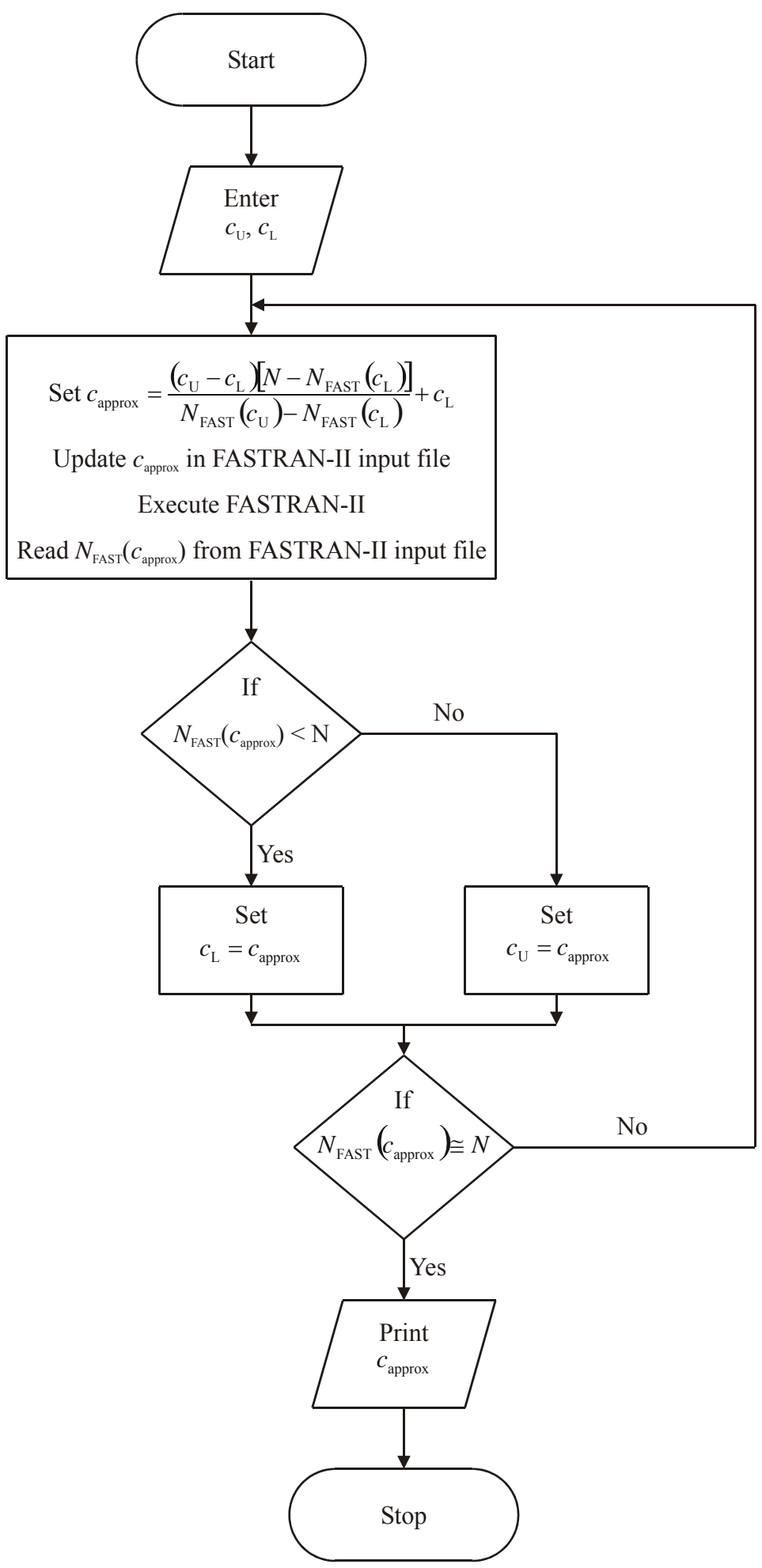

Figure 5.1 Flow chart depicting the iterative procedure involved in the calculation of equivalent initial flaw size 


\subsection{Parameter Relations}

Figures 5.2 to 5.6, depict values of equivalent initial flaw sizes calculated from experimentally measured fatigue lives replicated at each $S_{\max }$ level for the smooth unnotched specimen, the open-hole specimen and the $K_{\mathrm{t}}=5$ specimen. Two sets of equivalent initial flaw sizes have been reported at each $S_{\max }$ level; one set calculated using the corner crack assumption, $c_{0, \text { corner }}$, and another set calculated using the surface crack assumption, $c_{0, \text { surface }}$.

Figures 5.2 through 5.6 contain plots of equivalent initial flaw size, $c_{0}$, as a function of the level of maximum stress at failure, $S_{\max }$, for the smooth unnotched, open-hole and $K_{\mathrm{t}}=5$ specimens for the various mean stress levels of the experimental $S-N$ data. It can be seen from the smooth unnotched specimen case of Figure 5.2 at a mean stress, $S_{\text {mean }}$, of $144.790 \mathrm{MPa}$, the equivalent initial flaw sizes range from 23.9 to $48.2 \mu \mathrm{m}$ for the corner crack assumption and from 26.3 to $59.0 \mu \mathrm{m}$ for the surface crack assumption. 


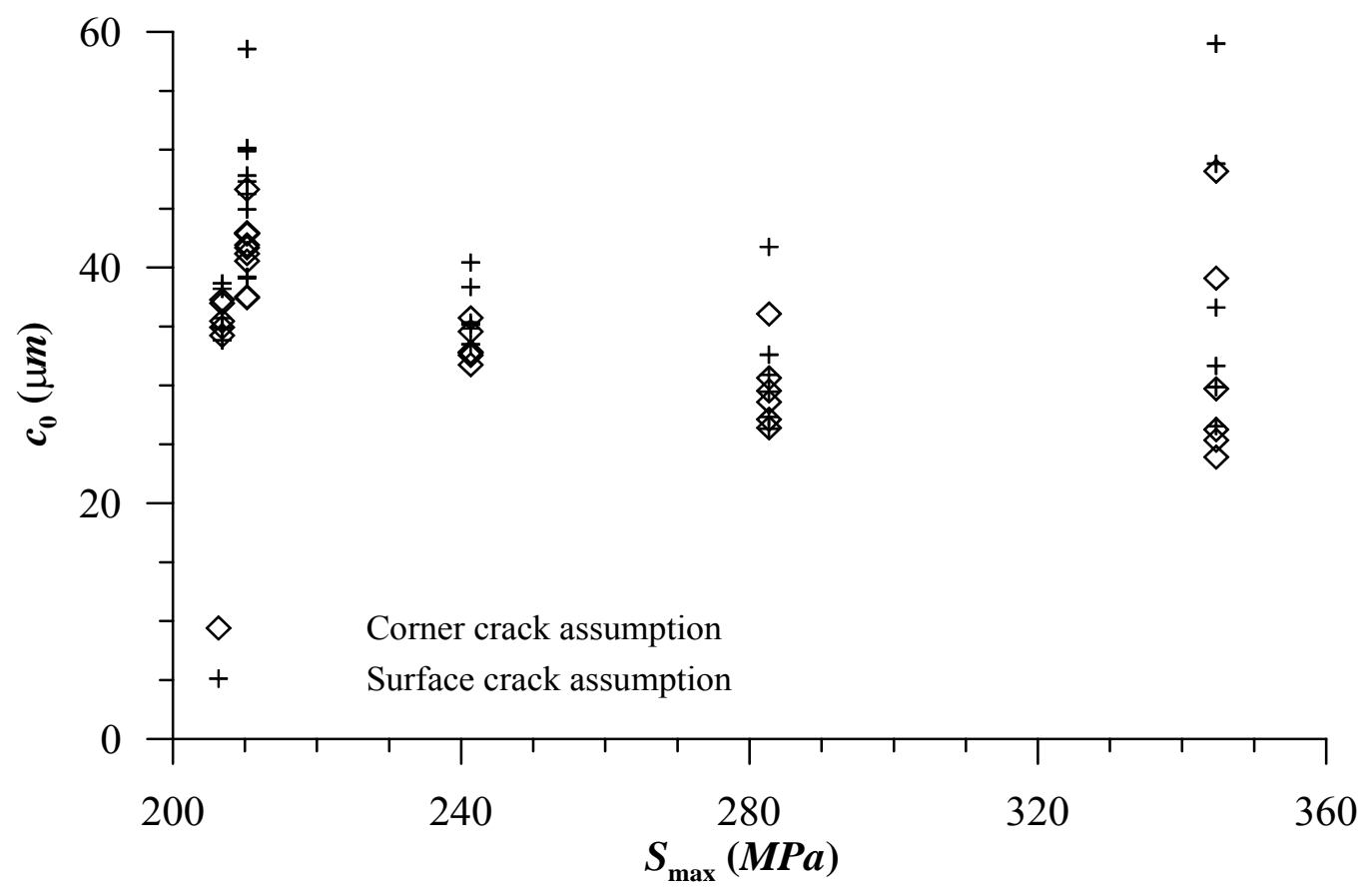

Figure 5.2 The relationship of $c_{0}$ as a function of the $S_{\max }$ for the smooth unnotched specimens with a $S_{\text {mean }}$ of $144.790 \mathrm{MPa}$

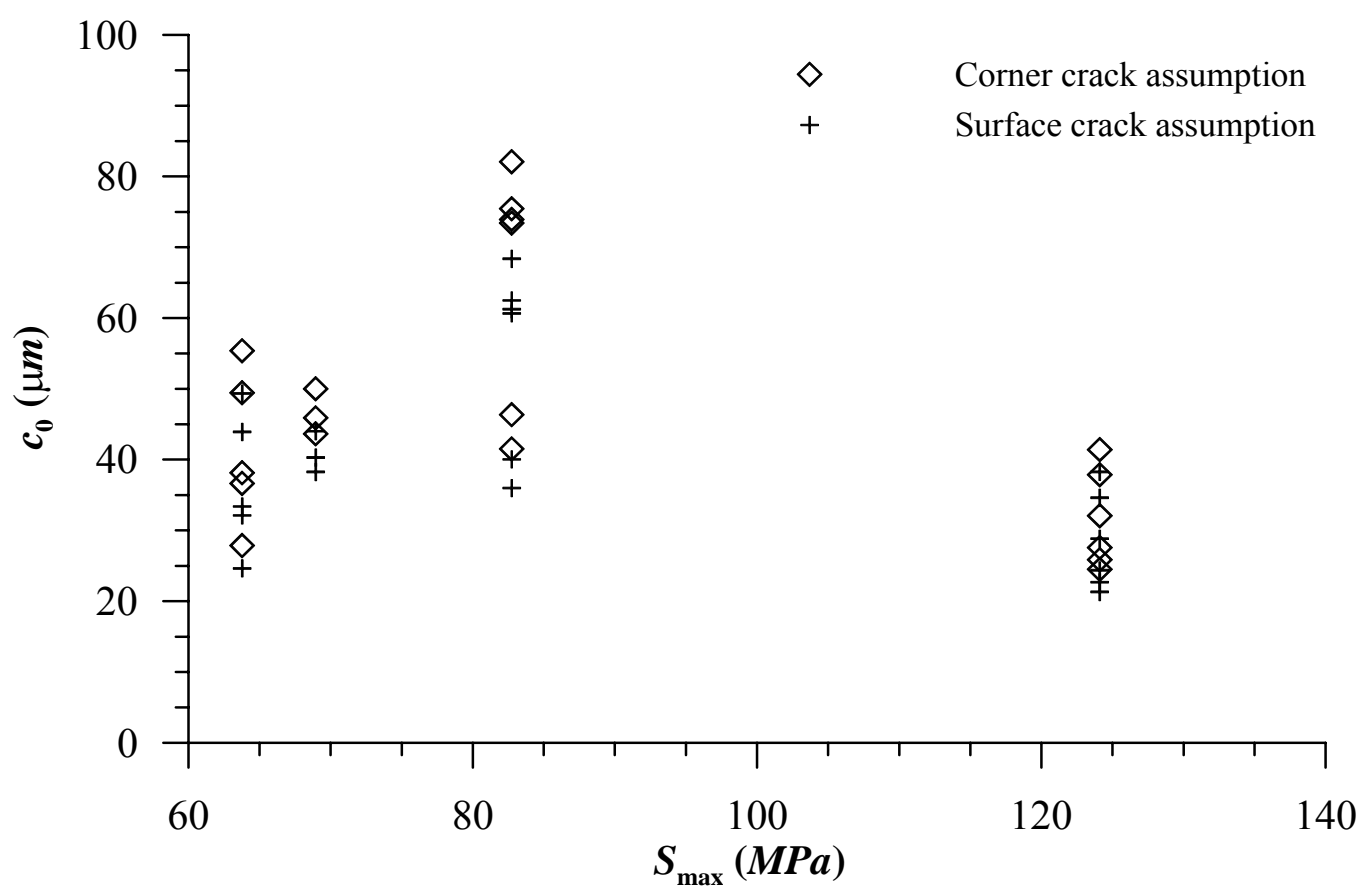

Figure 5.3 The relationship of $c_{0}$ as a function of the $S_{\max }$ for the open-hole specimens with a $S_{\text {mean }}$ of $20.684 \mathrm{MPa}$ 


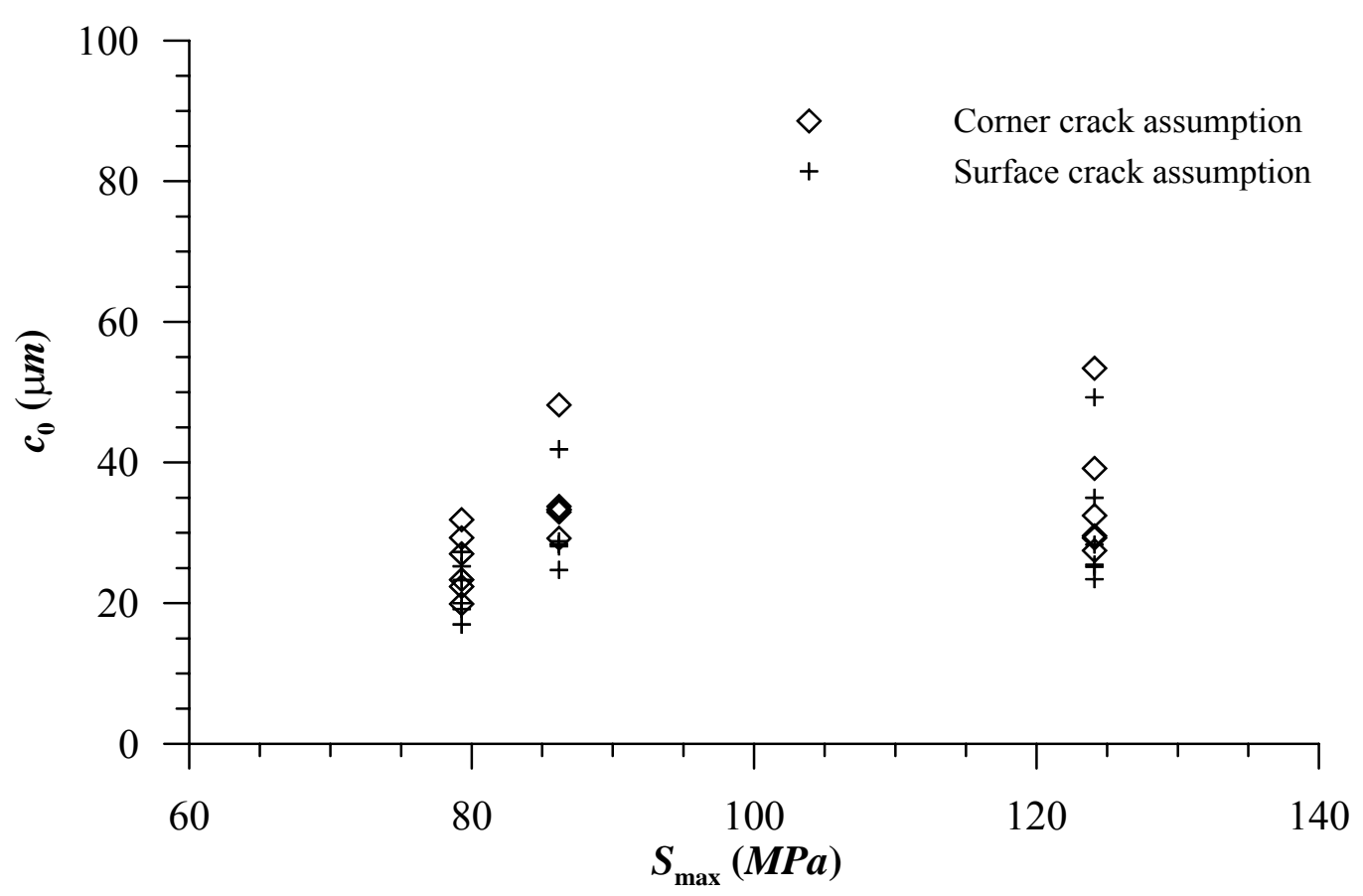

Figure 5.4 The relationship of $c_{0}$ as a function of the $S_{\max }$ for the open-hole specimens with a $S_{\text {mean }}$ of $41.369 \mathrm{MPa}$

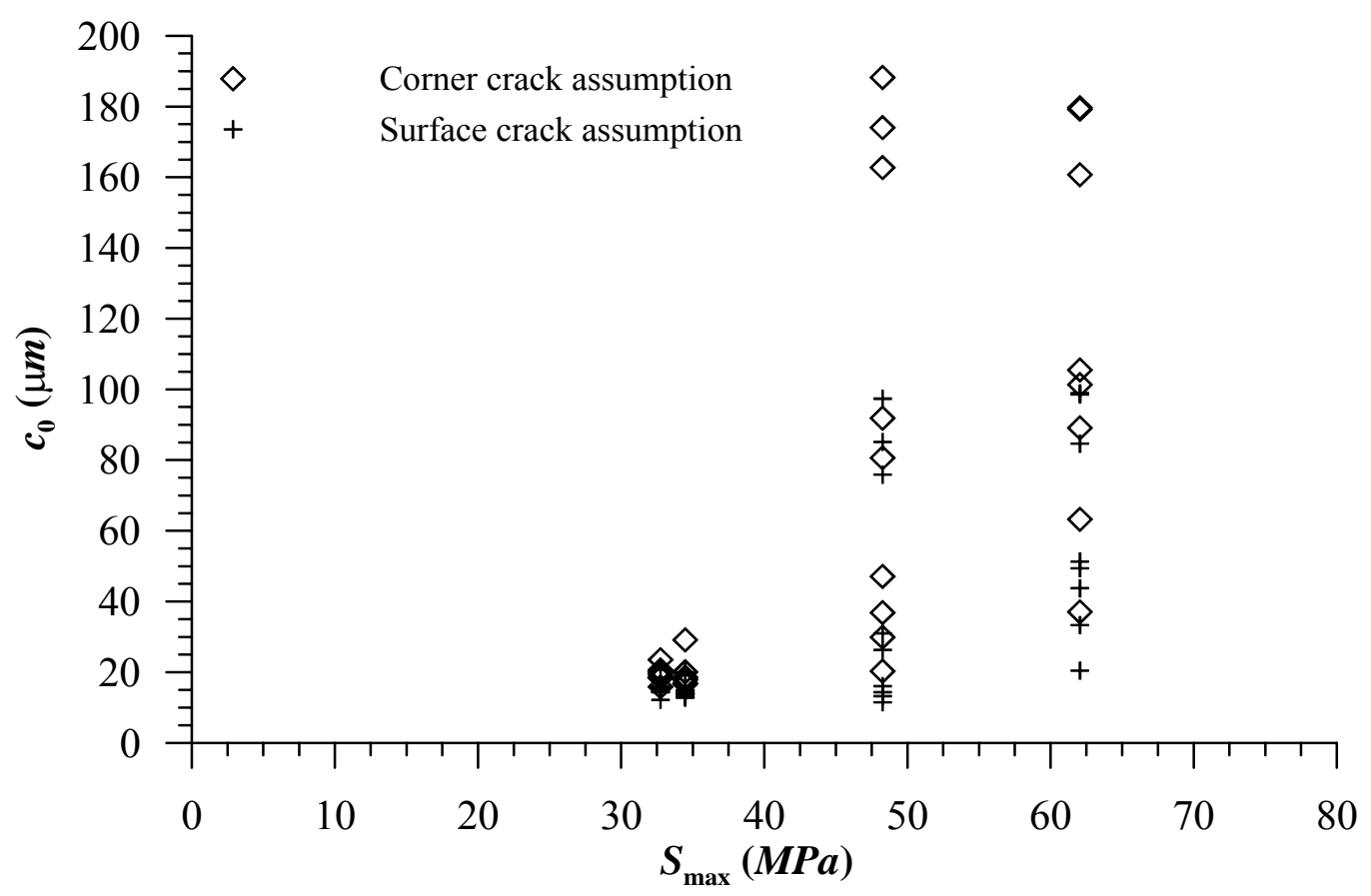

Figure 5.5 The relationship of $c_{0}$ as a function of the $S_{\max }$ for the $K_{\mathrm{t}}=5$ specimens with a $S_{\text {mean }}$ of $20.684 \mathrm{MPa}$ 


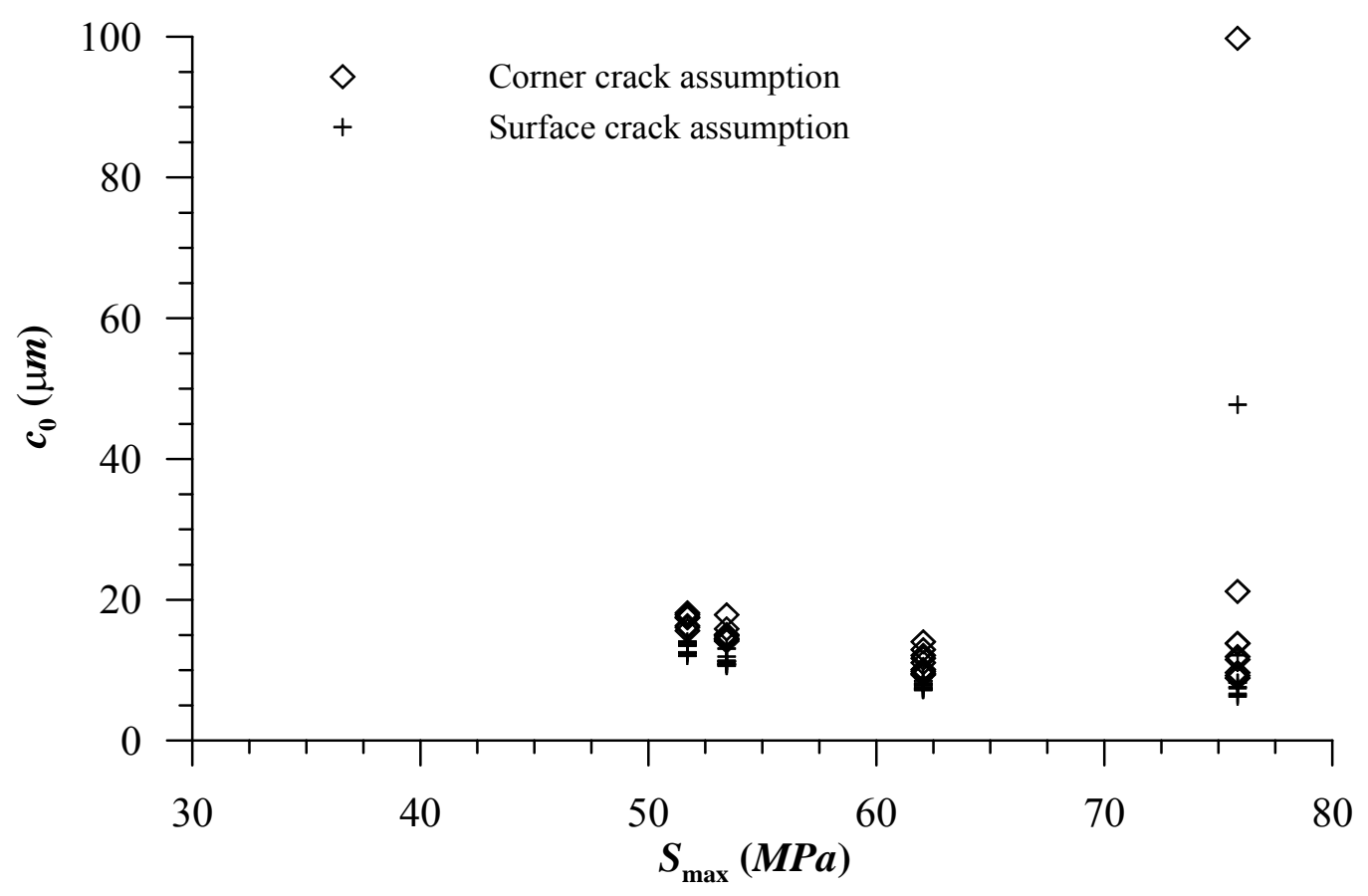

Figure 5.6 The relationship of $c_{0}$ as a function of the $S_{\max }$, for the $K_{\mathrm{t}}=5$ specimens with a $S_{\text {mean }}$ of $41.369 \mathrm{MPa}$

The data of Figure 5.3, associated with the open-hole specimens, indicates that for the case of a mean stress of $20.684 \mathrm{MPa}$, the equivalent initial flaw sizes fell in a range between 24.5 to $82.1 \mu \mathrm{m}$ for the corner crack assumption and from 21.3 to $68.4 \mu \mathrm{m}$ for the surface crack assumption. Whereas, for the $S_{\text {mean }}$ of $41.369 \mathrm{MPa}$ data of Figure 5.4, the flaw sizes ranged between 19.9 to $53.4 \mu \mathrm{m}$ for the assumption of an initial corner crack and from 17.0 to $49.3 \mu \mathrm{m}$ for the surface crack assumption.

The computed equivalent initial flaw size data presented in Figure 5.5 fell in a range between 15.8 to $188.2 \mu \mathrm{m}$ for the $K_{\mathrm{t}}=5$ specimen, at an $S_{\text {mean }}$ of $20.684 \mathrm{MPa}$ for an assumed initial corner crack and from 11.5 to $98.9 \mu \mathrm{m}$ for an assumed initial surface crack. Whereas, the data contained in Figure 5.6 for an $S_{\text {mean }}$ of $41.369 \mathrm{MPa}$ the equivalent initial flaw sizes ranged 
between 8.9 to $99.8 \mu \mathrm{m}$ for the corner crack assumption and from 6.3 to $47.7 \mu \mathrm{m}$ for the surface crack assumption.

Neither the smooth unnotched specimens nor the open-hole specimens exhibited a great deal of variability in the predicted equivalent initial flaw sizes as a function of the maximum stress. However, the $K_{\mathrm{t}}=5$ specimens showed pronounced variability in the flaw sizes over the range of maximum stresses, especially at the higher maximum stress levels.

With the exception of the smooth unnotched specimens, the equivalent initial flaw sizes calculated from a corner crack assumption were greater than the equivalent initial flaw sizes calculated from a surface crack assumption. The equivalent initial flaw sizes did not appear to conform to any particular pattern when they were plotted relative to the maximum stress at failure.

The objective in this phase of the study was to investigate how the equivalent initial flaw size mean, $\left(\bar{c}_{0, \text { corner }}\right.$ or $\left.\bar{c}_{0, \text { surface }}\right)$ and the equivalent initial flaw size standard deviation, $\left(s_{\text {corner }}\right.$ or $S_{\text {surface }}$ ) vary in relation with the $S_{\max }$ values. The first step of the investigation was to determine the equivalent initial flaw size mean and standard deviation respectively.

The equivalent initial flaw size mean, $\bar{c}_{0, \text { corner or surface }}$, at each $S_{\max }$ is defined by

$$
\bar{c}_{0, \text { corner or surface }}=\frac{\sum_{i=1}^{n}\left(c_{0, \text { corner or surface }}\right)_{i}}{n}
$$

where $\left(c_{0, \text { corner or surface }}\right)_{i}$ is the $i^{\text {th }}$ corner or surface assumed equivalent initial flaw size at each $S_{\max }$ level.

The equivalent initial flaw size standard deviation, $s_{\text {corner or surface }}$, at each $S_{\max }$ is defined by the following: 


$$
S_{\text {coner or o surface }}=\sqrt{\frac{\sum_{i=1}^{n}\left[\left(c_{0, \text { cormer or surface }}\right)_{i}-\bar{c}_{0, \text { comer or surface }}\right]^{2}}{n-1}}
$$

Once the equivalent initial flaw size mean and standard deviation values at each $S_{\text {max }}$ was determined, the next step was to investigate the relationship of $\bar{c}_{0}$ with respect to $S_{\max }$ and the relationship of $s$ with respect to $S_{\max }$ for smooth unnotched, open-hole, and $K_{\mathrm{t}}=5$ specimens for both assumed initial crack configurations. Figure 5.7 contains a compilation of plots of $\bar{c}_{0}$ as a function of $S_{\max }$ for both the assumed flaw types for all three specimen types. Figure 5.8 contains similar plots of $s$ as a function of $S_{\text {max }}$, also for both assumed flaw types and again for all three types of specimens.

From Figure 5.7 the equivalent initial flaw size mean associated with the assumed corner crack, $\bar{c}_{0, \text { comer }}$, for the smooth unnotched specimen type varied between 32.1 and $41.6 \mu \mathrm{m}$ while the equivalent initial flaw size mean for the assumed surface crack, $\bar{c}_{0, \text { surface }}$, varied between 31.4 and $47.3 \mu \mathrm{m}$. The mean values of the initial flaw size for the open-hole specimen type, $\bar{c}_{0, \text { cormer }}$, varied between 25.6 and $65.5 \mu \mathrm{m}$ for the corner crack assumption and the $\bar{c}_{0, \text { surface }}$ values varied between 22.0 and $47.3 \mu \mathrm{m}$ for the assumed initial surface flaws. As for the $K_{\mathrm{t}}=5$ specimen, the $\bar{c}_{0, \text { comer }}$ values varied between 11.2 and $114.5 \mu \mathrm{m}$ and the $\bar{c}_{0, \text { surface }}$ values varied between 7.9 and $60.0 \mu \mathrm{m}$. It is evident by the data of Figure 5.7 that no clear relationship emerged for $\bar{c}_{0}$ as a function of $S_{\max }$ that was valid for all three specimen types. 


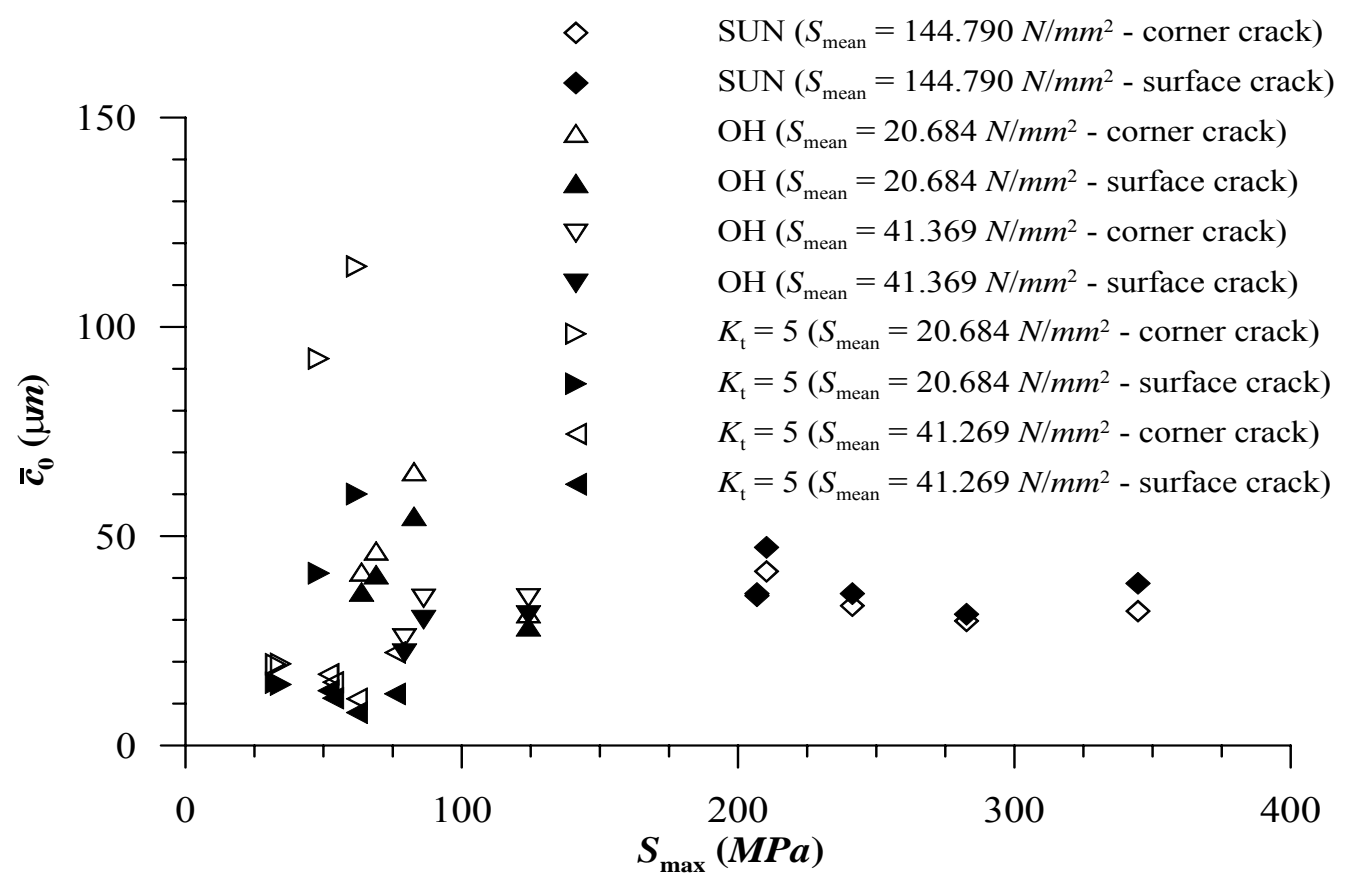

Figure 5.7 The relationship between $\bar{C}_{0}$ and $S_{\max }$ for smooth unnotched, open-hole and $K_{t}=5$ specimens

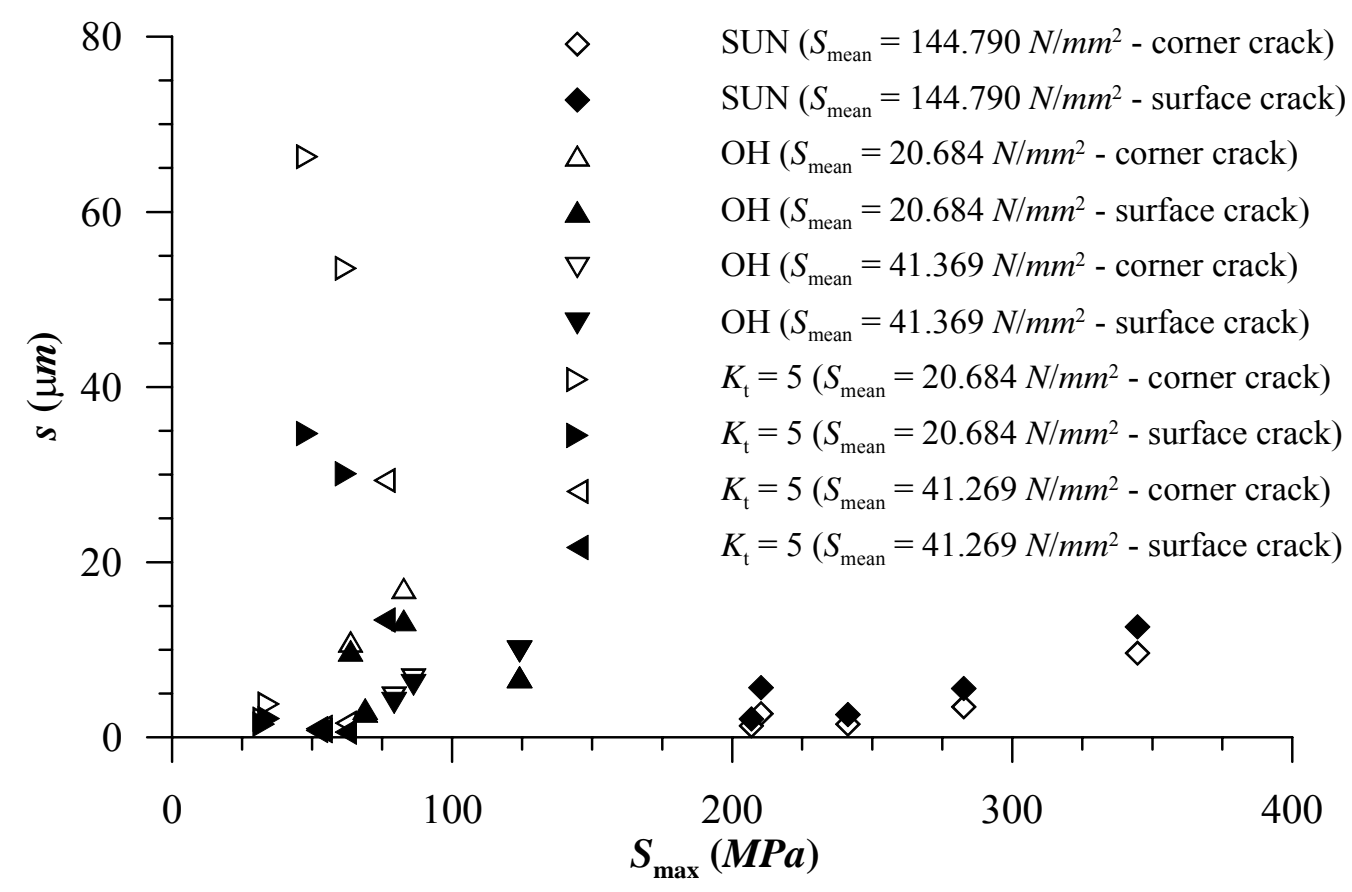

Figure 5.8 The relationship between $s$ and $S_{\max }$ for smooth unnotched, open-hole and $K_{t}=5$ specimens 
Similar to the $\bar{c}_{0}$ case, the standard deviation of the initial flaw sizes presented in Figure 5.8 did not suggest any clear relationship between $s$ and $S_{\max }$ for the three specimen types. The standard deviation values, $s_{\text {corner }}$ for the smooth unnotched specimen type for the assumed initial corner crack varied between 1.3 and $9.6 \mu \mathrm{m}$ while for the assumed surface crack the standard deviation values, $s_{\text {surface }}$, varied between 2.1 and $12.6 \mu \mathrm{m}$. The $s_{\text {corner }}$ for the open-hole specimens varied between 3.2 and $17.0 \mu \mathrm{m}$, and the $s_{\text {surface }}$ values varied between 2.9 and 13.4 $\mu m$. The $s_{\text {corner }}$ values for the $K_{\mathrm{t}}=5$ specimen varied between 1.0 and $66.3 \mu \mathrm{m}$ whereas the $S_{\text {surface }}$ values vary between 0.6 and $34.7 \mu \mathrm{m}$.

Since neither $\bar{c}_{0}$ nor the $s$ data presented in Figure 5.7 and 5.8 indicated a clear relationship with $S_{\max }$ it was necessary to transform the dependent variables $\bar{c}_{0}$ and $s$ into variables that could exhibit a distinct pattern with respect to $S_{\max }$. This variable transformation approach was neither a required nor a standard procedure; it merely simplified some of the process involved in establishing the statistical relation through multiple regression analyses. Because of the complex relations that sometimes exist between independent, or regressor variables, and the response variables; finding a good relation between these variables can be tedious and involved. Some of these complexities can be simplified by introducing the variable transformation approach.

After considerable trial and error examinations of various combination of variables, a set of transformation equations were introduced for $\bar{c}_{0}$ and $s$. The new transformed variables were called the equivalent mean, $\bar{c}_{0}^{\mathrm{eq}}$, and equivalent standard deviation, $s^{\mathrm{eq}}$. 
The equivalent initial flaw size equivalent mean, $\bar{c}_{0 \text {,corner or surface, }}^{\text {eq }}$ at each $S_{\max }$ level was defined by

$$
\bar{C}_{0, \text { corner or surface }}^{\text {eq }}=\frac{-\log \left(\bar{C}_{0, \text { corner or surface }}\right)}{S_{\max }}
$$

and the equivalent initial flaw size equivalent standard deviation, $S_{\text {corner or surface }}^{\text {eq }}$ at each $S_{\max }$ level was defined by

$$
S_{\text {corner or surface }}^{\mathrm{eq}}=\frac{-\log \left(S_{\text {corner or surface }}\right)}{S_{\text {max }}}
$$

Figures 5.9 and 5.10 depict $\bar{c}_{0}^{\text {eq }}$ as a function of $S_{\max }$ and $s^{\text {eq }}$ as a function of $S_{\max }$ respectively for smooth unnotched, open-hole specimens, and $K_{t}=5$ specimens for both assumed initial crack configurations.

From the plotted values of Figure 5.9 the equivalent mean, $\bar{c}_{0}^{\mathrm{eq}}$ values for the smooth unnotched specimen type varied between 0.013 and 0.021 for both assumed initial flaw types. The equivalent mean values for the open-hole specimen type varied between 0.036 and 0.059 for both assumed initial flaw sizes. As for the $K_{\mathrm{t}}=5$ specimen, the equivalent mean values for the corner crack configuration, $\bar{c}_{0, \text { corner }}^{\text {eq }}$ varied between 0.064 and 0.092 whereas for the surface crack configuration $\bar{c}_{0, \text { surface }}^{\mathrm{eq}}$ varied between 0.068 and 0.094 . From observation of Figure 5.9 it is clear that $\bar{C}_{0}^{\text {eq }}$ is inversely proportional to $S_{\max }$ for both crack configurations as it must be from equation (5.5). The equivalent mean values for the assumed corner and surface were almost identical for both the smooth unnotched and open-hole specimen types. Another interesting observation from Figure 5.9 is that the $\bar{c}_{0}^{\text {eq }}$ values for each specimen type occupied a specific range that did not overlap the ranges of the other specimen types. 


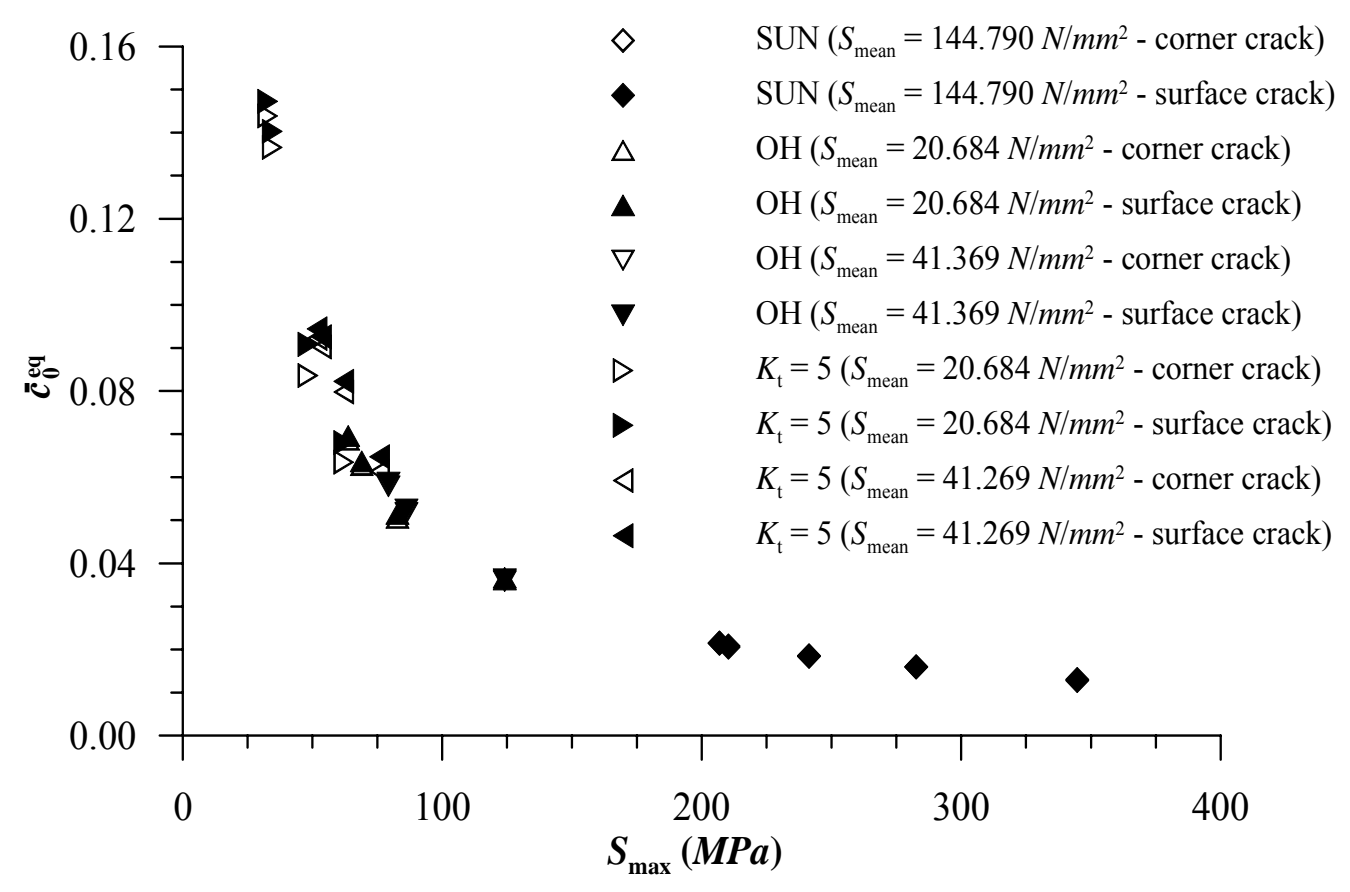

Figure 5.9 The relationship between $\bar{C}_{0}^{\mathrm{eq}}$ and $S_{\max }$ for smooth unnotched, open-hole and $K_{t}=5$ specimens

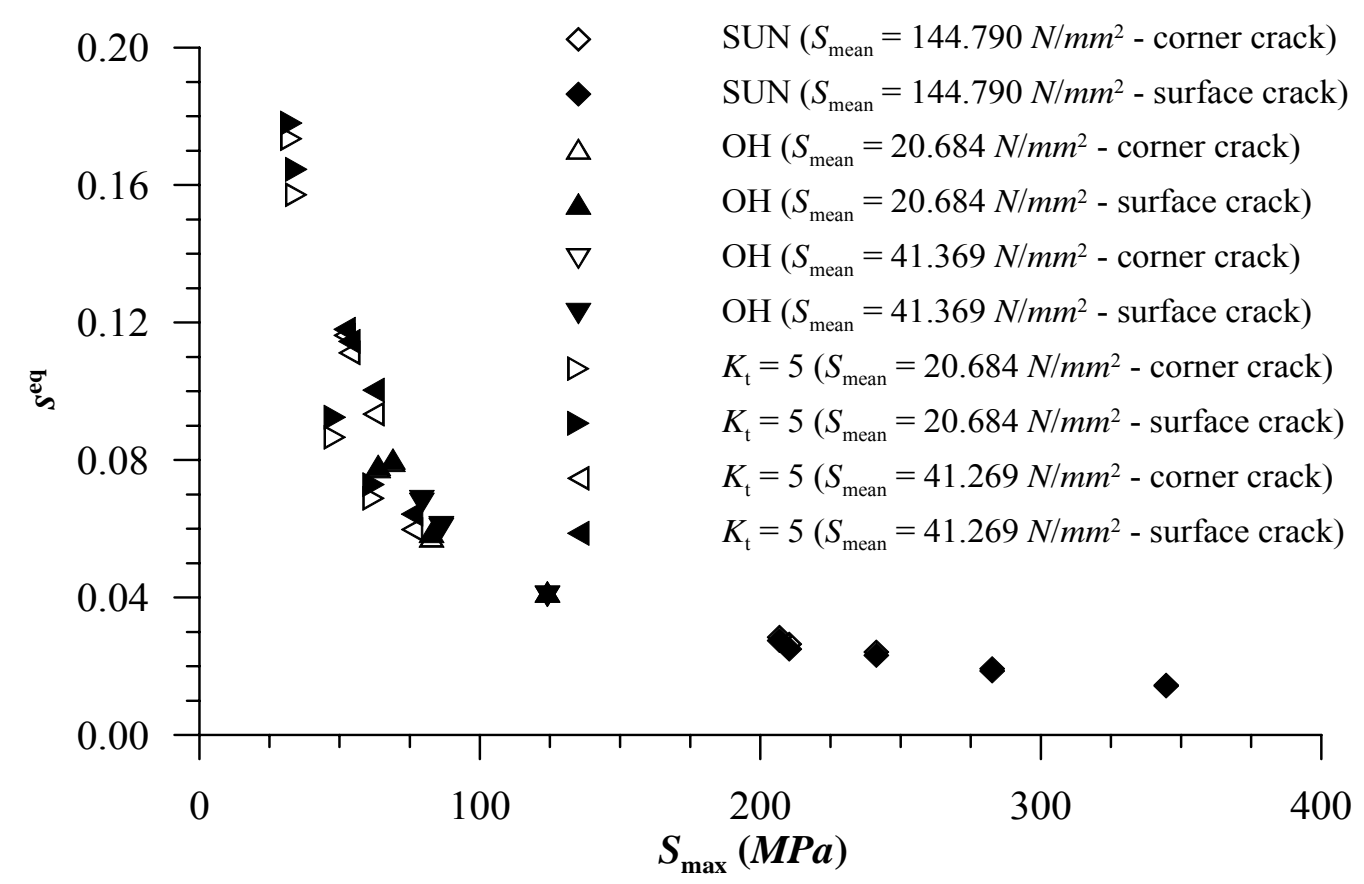

Figure 5.10 The relationship between $S^{\mathrm{eq}}$ and $S_{\max }$ for smooth unnotched, open-hole and $K_{t}=5$ specimens 
Similar observations apply to the plotted values of $s^{\text {eq }}$ in Figure 5.10. For the smooth unnotched specimen the equivalent standard deviation, $s^{\text {eq }}$, values ranged between 0.014 and 0.028 for both assumed initial flaw types. In the case of the open-hole specimen the $s^{\text {eq }}$ values varied between 0.042 and 0.068 for both assumed initial flaw types. As for the $K_{\mathrm{t}}=5$ specimen, the $s_{\text {corner }}^{\mathrm{eq}}$ values ranged between 0.069 and 0.116 whereas the $S_{\text {surface }}^{\mathrm{eq}}$ values varied between 0.073 and 0.118 . As seen in equation (5.6), $\mathrm{s}^{\mathrm{eq}}$ varied inversely proportional to $S_{\max }$ for both crack configurations. Similar to the equivalent mean case, the equivalent standard deviation values for the assumed corner and surface were almost identical for both the smooth unnotched and open-hole specimens. Also the $s^{\text {eq }}$ values for each specimen type seemed to occupy specific range according to specimen types and with no overlap amongst each other.

Having established a pattern of behavior for both the $\bar{c}_{0}^{\mathrm{eq}}$ and $s^{\mathrm{eq}}$ relative to $S_{\max }$, multiple regression analyses could be performed to determine a statistical relation between specimen and crack geometry and the parameters associated with the applied load.

\subsection{Multiple Regression Analyses}

The effects of $K_{\mathrm{tg}}, w_{\mathrm{n}}, S_{\text {mean }}$, and $S_{\max }$ on $\bar{c}_{0}^{\mathrm{eq}}$ and $s^{\mathrm{eq}}$ were investigated in this part of the study for both initial crack configurations. Multiple regression analyses, similar to those of Reference [55-56], were performed using the STATGRAPHICS Plus software [57] in order to find the following relations:

$$
\begin{gathered}
\bar{C}_{0, \text { corner or surface }}^{\mathrm{eq}}=f\left(S_{\text {max }}, S_{\text {mean }}, K_{\mathrm{tg}}, w_{\mathrm{n}}\right) \\
S_{\text {corner or surface }}^{\text {eq }}=f\left(S_{\text {max }}, S_{\text {mean }}, K_{\mathrm{tg}}, w_{\mathrm{n}}\right)
\end{gathered}
$$


The remainder of this section is devoted to the detailed explanation of the multiple regression approach useful for this investigation. Consider a single dependent variable, or response, $y$, that depends on $k$ independent variables, or regressor variables, $x_{1}, x_{2}, \ldots, x_{k}$. The relationship between the response and the regressor variables is characterized by a mathematical relationship called a multiple regression model. The term "multiple" denotes that there is more than one regressor variable. The type of regression model used in this study is called the generalized linear model also referred to as the multiple linear regression model. In the generalized linear model, the response variable is always a linear function of the regressor variables.

Consider the following multiple linear regression model that describes the relationship with two regressor variables $\left(x_{1}\right.$ and $\left.x_{2}\right)$. The model takes the form

$$
y=\beta_{0}+\beta_{1} x_{1}+\beta_{2} x_{2}+\varepsilon
$$

The parameters $\beta_{0}, \beta_{1}$, and $\beta_{2}$ are called the regression coefficients and $\varepsilon$ is the random error. A basic assumption associated with the model is that for a set of observations, $\left(y_{i}, x_{1(i)}, x_{2(i)}\right)$ the mean value of $y$ evaluated at given values of $x_{1}$ and $x_{2}$ traces a plane in the 3-D $x_{1}, x_{2}$, and $y$ space and that the given set of data points deviate about this plane of means by a random amount equal to $\varepsilon$, the random error. The parameter $\beta_{0}$ defines the intercept of the plane with the $y$-axis. The parameters $\beta_{1}$ and $\beta_{2}$ are sometimes called the partial regression coefficients, because $\beta_{1}$ represents the expected change in $y$ per unit change in $x_{1}$, when $x_{2}$ is held constant; and, $\beta_{2}$ represents the expected change in $y$ per unit change in $x_{2}$, when $x_{1}$ is held constant. If one assumes that the mean values of $y$ deviate above and below the plane (positive deviations, 
and negative deviations), and with the expected value of $\varepsilon, E(\varepsilon)=0$, then the expected value of $y, E(y)$ is given by

$$
E(y)=E\left(\beta_{0}+\beta_{1} x_{1}+\beta_{2} x_{2}+\varepsilon\right)
$$

Let $\hat{y}$ be defined to be an estimator of the mean value of $y, E(y)$, and a predictor of some future value of $y$, then,

$$
\begin{gathered}
\hat{y}=\hat{\beta}_{0}+\hat{\beta}_{1} x_{1}+\hat{\beta}_{2} x_{2}+E(\varepsilon) \\
\hat{y}=\hat{\beta}_{0}+\hat{\beta}_{1} x_{1}+\hat{\beta}_{2} x_{2}
\end{gathered}
$$

where $\hat{\beta}_{0}, \hat{\beta}_{1}$ and $\hat{\beta}_{2}$ are estimators of $\beta_{0}, \beta_{1}$ and $\beta_{2}$ and $x_{1}$, and $x_{2}$ are regressor variables. In deriving equation (5.10) it is assumed that the random error, $\varepsilon$, is to have a normal distribution with mean zero and a constant variance. Using a set of observed response data, $y_{i}$ at specific pairs of $x_{1(i)}$, and $x_{2(i)}$ observed regressor data, the corresponding estimates of $\hat{\beta}_{0}, \hat{\beta}_{1}$ and $\hat{\beta}_{2}$ of the regression parameters can be determined using a minimization technique known as the least squares method. Note that the parameter estimates are not unique since they are functions of the particular set of experimental observations.

In general, the response variable $y$ may be related to $k$ regressor variables. For example, the model

$$
\hat{y}=\hat{\beta}_{0}+\hat{\beta}_{1} x_{1}+\hat{\beta}_{2} x_{2}+\cdots+\hat{\beta}_{k} x_{k}
$$

is called a multiple linear regression model with $k$ regressor variables.

Models those are more complex than equation (5.10) may often be analyzed by multiple linear regression techniques. For example, consider adding an additional interaction term, $\hat{\beta}_{12} x_{1} x_{2}$ to the first-order model in two variables, equation (5.10), such that 


$$
\hat{y}=\hat{\beta}_{0}+\hat{\beta}_{1} x_{1}+\hat{\beta}_{2} x_{2}+\hat{\beta}_{12} x_{1} x_{2}
$$

Define a new parameter $x_{3}$ such that $x_{3} \equiv x_{1} x_{2}$ and $\hat{\beta}_{3} \equiv \hat{\beta}_{12}$, then equation (5.12) can be written

as

$$
\hat{y}=\hat{\beta}_{0}+\hat{\beta}_{1} x_{1}+\hat{\beta}_{2} x_{2}+\hat{\beta}_{3} x_{3}
$$

which is a standard multiple linear regression model with three regressor variables.

As another example, consider the second-order response surface model in two variables with the following form:

$$
\hat{y}=\hat{\beta}_{0}+\hat{\beta}_{1} x_{1}+\hat{\beta}_{2} x_{2}+\hat{\beta}_{11} x_{1}^{2}+\hat{\beta}_{22} x_{2}^{2}+\hat{\beta}_{12} x_{1} x_{2}
$$

Again, define new parameters $x_{3} \equiv x_{1}^{2}, x_{4} \equiv x_{2}^{2}, x_{5} \equiv x_{1} x_{2}, \hat{\beta}_{3} \equiv \hat{\beta}_{11}, \hat{\beta}_{4} \equiv \hat{\beta}_{22}$, and $\hat{\beta}_{5} \equiv \hat{\beta}_{12}$, then this becomes

$$
\hat{y}=\hat{\beta}_{0}+\hat{\beta}_{1} x_{1}+\hat{\beta}_{2} x_{2}+\hat{\beta}_{3} x_{3}+\hat{\beta}_{4} x_{4}+\hat{\beta}_{5} x_{5}
$$

which is a linear regression model with five regressor variables.

Multiple regression analyses can be time consuming and tedious. In this study the regression analyses were performed using the commercially available software, STATGRAPHICS Plus. Using the geometric and loading condition data for the smooth unnotched specimen, the open-hole specimen, and the $K_{\mathrm{t}}=5$ specimen the following relations were obtained with STATGRAPHICS Plus for the estimated equivalent mean, $\hat{\bar{C}}_{0}^{\text {eq }}$, and the estimated equivalent standard deviation, $\hat{s}^{\text {eq }}$ :

$$
\begin{gathered}
\hat{\bar{C}}_{0, \text { corner }}^{\mathrm{eq}}=0.000453704+8.79502 S_{\operatorname{mean}}^{0.074} S_{\max }^{-1.056} K_{\mathrm{tg}}^{0.024}-5.31026 S_{\max }^{-0.981} w_{\mathrm{n}}^{0.045} \\
\hat{S}_{\text {corner }}^{\mathrm{eq}}=-0.000668527+10.2976 S_{\operatorname{mean}}^{0.146} S_{\max }^{-1.239} K_{\mathrm{tg}}^{-0.108}
\end{gathered}
$$

for an assumed initial corner crack, and 


$$
\begin{aligned}
& \hat{\bar{C}}_{0, \text { surface }}^{\text {eq }}=0.000200679+7.45709 S_{\operatorname{mean}}^{0.067} S_{\max }^{-1.035} K_{\mathrm{tg}}^{0.060}-5.49433 S_{\max }^{-0.975} w_{\mathrm{n}}^{0.093} \\
& \hat{S}_{\text {surface }}^{\mathrm{eq}}=-0.000352877+8.80526 S_{\text {mean }}^{0.136} S_{\max }^{-1.210} K_{\mathrm{tg}}^{-0.050}
\end{aligned}
$$

for an assumed initial surface crack. Each statistical relation was obtained using twenty sets of data, that is $n=20$.

Each equivalent mean response variable for both assumed crack cases expressed in equations (5.16) and (5.18) was a function of two regressor variables, while each equivalent standard deviation response variable expressed in equations (5.17) and (5.19) was a function of only one regressor variable. As illustrated in equations (5.16) to (5.19), the regressor variables are complicated combinations of geometric and loading parameters. The regressor variables were obtained by experimenting with various combinations of geometric and loading parameters.

Before we accepted the validity of these statistical relations it was important that we conduct some tests on the multiple regression models in order to be certain that the models were feasible and valid. The most common verification procedures are residual analysis and hypothesis testing. Appendix $\mathrm{C}$ contains multiple regression analysis and analysis of variance report for equations (5.16) through (5.19) obtained from STATGRAPHICS Plus which was useful for the verification procedures. Some of the fundamental concepts of the verification process will be detailed in the next few paragraphs.

\subsection{Residual Analysis of Regression Models}

An assumption associated with multiple regression analyses is that the experimental random errors are uncorrelated and have a normal distribution with zero mean and a constant variance. Another implicit assumption is that the chosen multiple regression model must be 
capable of representing the parameters of interest. Therefore, these assumptions must be verified in order to have confidence in the empirical modeling approach.

The average of the response variable, $y$ is defined by the expression

$$
\bar{y}=\frac{\sum_{i=1}^{n} y_{i}}{n}
$$

where $n$ is the total number of observations (20 in this study) and $y_{i}$ is the observed or measured response data. The total sum of squares, $S S_{\text {total }}$, is the summation of the squares of the differences of the observed response and the average value of the observed response, thus

$$
S S_{\text {total }}=\sum_{i=1}^{n}\left(y_{i}-\bar{y}\right)^{2}
$$

The difference between the predicted variable, $\hat{y}_{i}$, and the average of the response value, $\bar{y}$, may be used to quantify the variation of the regression model. The sum of the squares of these differences is termed the regression sum of squares, defined by

$$
S S_{\text {regression }}=\sum_{i=1}^{n}\left(\hat{y}_{i}-\bar{y}\right)^{2}
$$

It can be shown that

$$
S S_{\text {total }}=S S_{\text {regression }}+S S_{\text {error }}
$$

where $S S_{\text {error }}$ is referred to as the error sum of squares, and is defined by the expression

$$
S S_{\text {error }}=\sum_{i=1}^{n}\left(y_{i}-\hat{y}_{i}\right)^{2}
$$

Consequently it is clear from equation (5.23) that the total variation due to the difference between the observed response and predicted variable has a contribution from the regression model and a contribution from the residual error. 
The total deviation of the $n$ observations from the average value must sum to zero, hence $\sum_{i=1}^{n}\left(y_{i}-\bar{y}\right)=0$. This condition can be met by knowing only $n-1$ values of $y$ and $\bar{y}$. Therefore, in the determination of $S S_{\text {total }}$ it is implied that only $n-1$ of the contributing $\left(y_{i}-\bar{y}\right)$ elements are independent. Hence the total sum of squares is said to have $n-1$ degrees of freedom because one degree of freedom was lost due to the calculation of $\bar{y}$. In statistics, the term "degree of freedom" is a measure of the number of independent items of information on which the precision of a parameter estimate is based. The degree of freedom for an estimate equals the number of observations minus the number of additional parameters estimated for that calculation. As more parameters have to be estimated, the degrees of freedom available decreases. The number of degrees of freedom can be thought of as the number of observations which are freely available to vary, given the additional parameters estimated.

In evaluating the error sum of squares, $S S_{\text {error }}$, a total of $k+1$ degrees of freedom are lost, where $k$ is number of regressor coefficients $\beta_{1}, \beta_{2}, \ldots, \beta_{k}$, therefore resulting in a model of $n-k-1$ degrees of freedom. That is, one degree of freedom is lost in the estimation of each model parameters $\hat{\beta}_{0}$ through $\hat{\beta}_{k}$. These estimated model parameters are used in the calculation of the error sum of squares, hence $k$ degrees of freedom are lost in the calculation of the error sum of squares. Since degrees of freedom are additive, the degrees of freedom associated with the regression sum of squares, $S S_{\text {regression }}$ is $k$ (degrees of freedom associated with $S S_{\text {total }}$ minus the degrees of freedom associated with $S S_{\text {error }}$ ).

The mean sums of squares are defined by dividing each of the sum of squares by their respective number of degrees of freedom. Therefore, the mean total sum of squares, MSS $_{\text {total }}$, 
mean regression sum of squares, $M S S_{\text {regressionl }}$, and mean error sum of squares, $M S S_{\text {error }}$ can be expressed as

$$
\begin{aligned}
M S S_{\text {total }} & =\frac{S S_{\text {total }}}{n-1} \\
M S S_{\text {regression }} & =\frac{S S_{\text {regression }}}{k} \\
M S S_{\text {error }} & =\frac{S S_{\text {error }}}{n-k-1}
\end{aligned}
$$

A residual analysis is a procedure designed to check the assumption that model errors are normally distributed with a constant variance. For this purpose, a normal probability plot of residuals was constructed. A normal probability plot is used to investigate whether process data exhibit a normal, or Gaussian, distribution. If the distribution is close to a normal distribution, the plotted points will lie close to a straight line. Systematic deviations from the straight line indicate a non-normal distribution.

The following steps were involved in the construction of a normal probability plot of residuals. First the $i^{\text {th }}$ residual, $e_{i}$, was calculated from the following expression

$$
e_{i}=y_{i}-\hat{y}_{i}
$$

where $y_{i}$ is the $i^{\text {th }}$ observed response data and $\hat{y}_{i}$ is the corresponding predicted value obtained from the regression model. Expanding equation (5.28) for a multiple regression model with $k$ regressor coefficients leads to

$$
e_{i}=y_{i}-\left[\hat{\beta}_{0}+\hat{\beta}_{1} x_{1(i)}+\hat{\beta}_{2} x_{2(i)}+\cdots+\hat{\beta}_{k} x_{k(i)}\right]
$$

where $x_{1(i)}, x_{2(i)}, \ldots, x_{k(i)}$ are the $i^{\text {th }}$ observed regressor data corresponding to $y_{i}$. The second step was to list the data set of residuals in an ascending order. The newly ordered data is 
commonly termed as "ranked data". The tail area of the $z$-distribution (standard normal distribution) corresponding to each ranked $i^{\text {th }}$ residual data, $A_{i}$, was determined from the following expression

$$
A_{i}=\frac{i-0.375}{n+0.250}
$$

where $n$ is total number of observations or sample size. The expected value of the $i^{\text {th }}$ residual data, $E\left(e_{i}\right)$ is

$$
E\left(e_{i}\right)=\sqrt{M S S_{\text {error }}}\left[P\left(z \leq A_{i}\right)\right]
$$

where $P\left(z \leq A_{i}\right)$ is the probability of $z$ being less than or equal to $A_{i}$, where $z$ is a random variable that has a standard normal distribution and $M S S_{\text {error }}$ is the mean error sum of squares of the residuals. Equations (5.30) and (5.31) were obtained from Reference [59]. The normal probability plot was constructed by plotting the ranked residual, $e_{i}$, on the vertical axis and the corresponding expected residual, $E\left(e_{i}\right)$ on the horizontal axis.

Figures 5.11 to 5.14 contain the normal probability plots of the residuals for equivalent mean and equivalent standard deviation of this study. In each case the plotted data points track the trend line relatively well thus validating the normality condition of the error. The slope and $y$-intercept of the trend line were obtained from linear regression analysis; by regressing the ranked residuals, $e$, on the corresponding expected residuals, $E(e)$. 


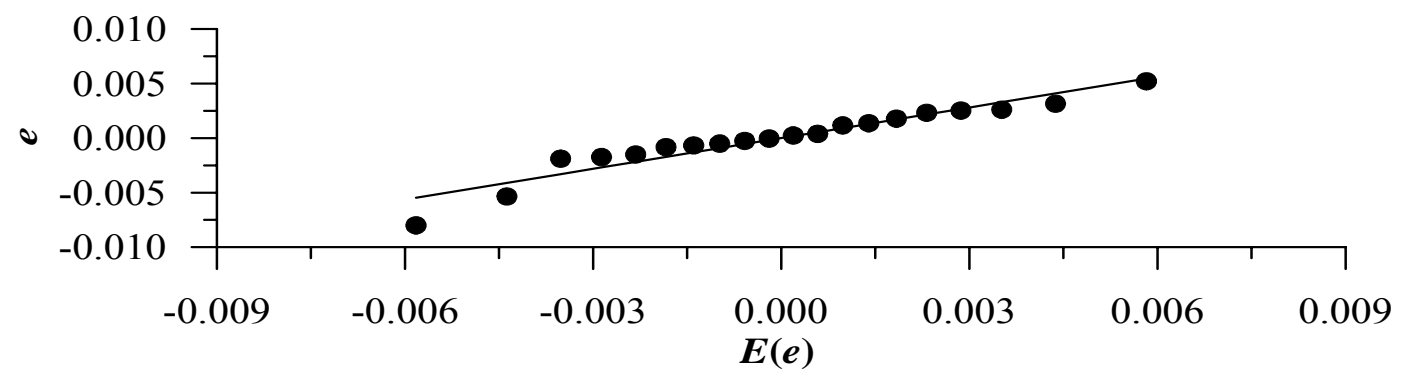

Figure 5.11 Residual normal probability plot for the $\hat{\bar{C}}_{0, \text { corner }}^{\text {eq }}$ model

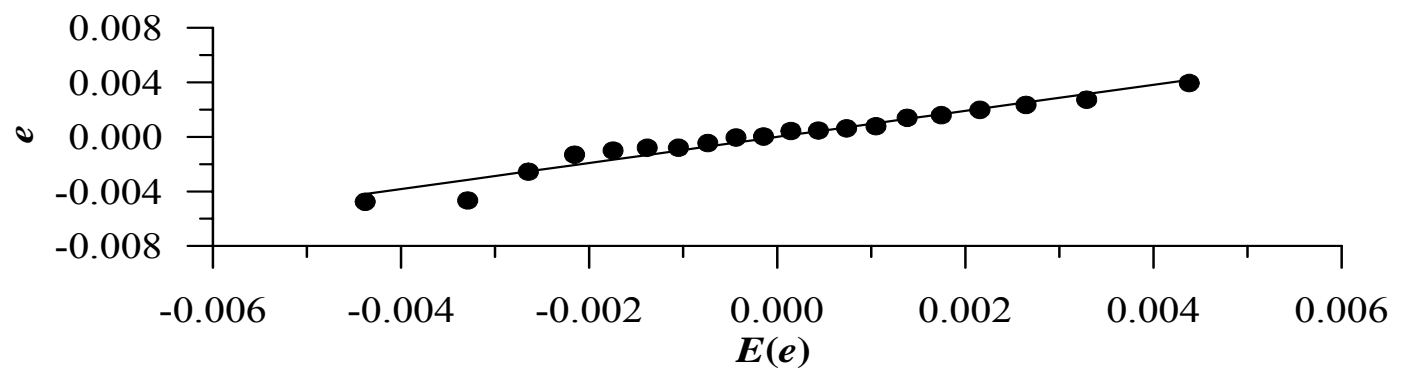

Figure 5.12 Residual normal probability plot for the $\hat{\bar{C}}_{0 \text {,surface }}^{\text {eq }}$ model

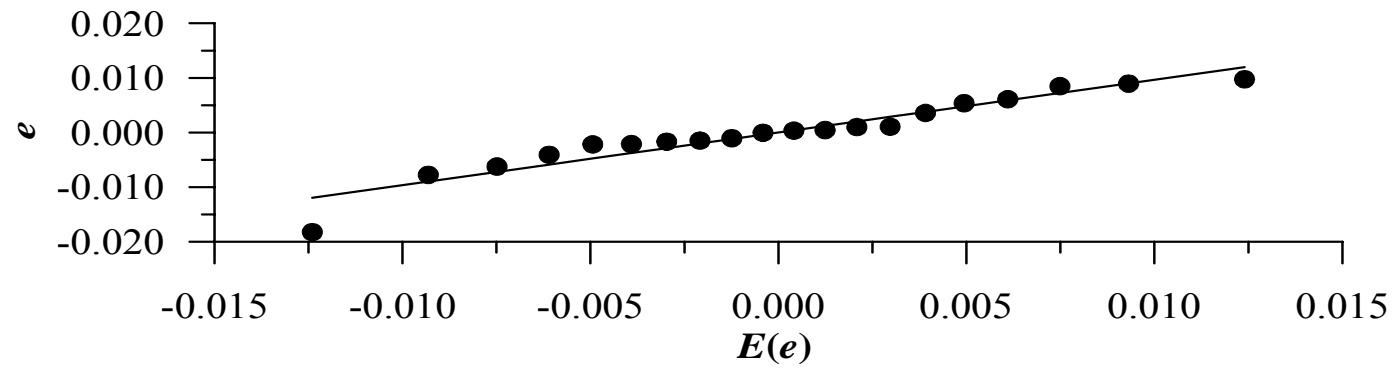

Figure 5.13 Residual normal probability plot for the $\hat{s}_{\text {corner }}^{\text {eq }}$ model

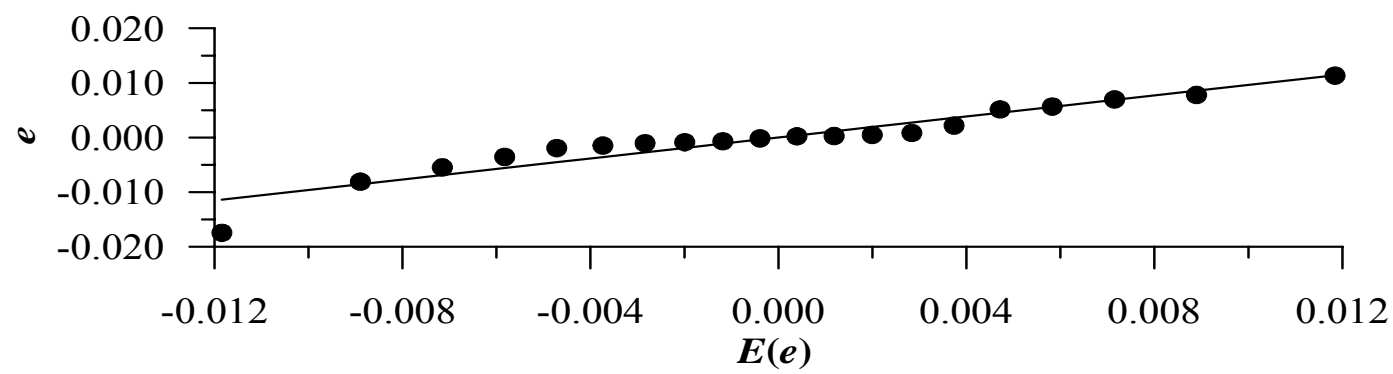

Figure 5.14 Residual normal probability plot for the $\hat{s}_{\text {surface }}^{\mathrm{eq}}$ model 
The notion of a constant variance of the distribution can be verified by constructing a plot of studentized residual, $e_{\text {studentized }}$, as a function of the estimated response value, $\hat{y}$. The variance of the $i^{\text {th }}$ residual, $V\left(e_{i}\right)$, is a function of the observed regressor data and can be defined by

$$
V\left(e_{i}\right)=M S S_{\text {error }}\left(1-h_{i i}\right)
$$

$M S S_{\text {error }}$ is the mean error sum of squares for the residuals and $h_{i i}\left(0<h_{i i} \leq 1\right)$ is the $i^{\text {th }}$ diagonal element of the square matrix, $\boldsymbol{H}$, defined by the matrix expression

$$
\boldsymbol{H} \equiv \boldsymbol{x} \cdot\left(\boldsymbol{x}^{\mathrm{T}} \cdot \boldsymbol{x}\right)^{-1} \cdot \boldsymbol{x}^{\mathrm{T}}
$$

where $\boldsymbol{x}$ is given by

$$
\boldsymbol{x}=\left[\begin{array}{ccccc}
1 & x_{1(1)} & x_{2(1)} & \ldots & x_{k(1)} \\
1 & x_{1(2)} & x_{2(2)} & \ldots & x_{k(1)} \\
\vdots & \vdots & \vdots & \vdots & \vdots \\
1 & x_{1(n)} & x_{2(n)} & \ldots & x_{k(n)}
\end{array}\right]
$$

where $x_{j(i)}$ is the $i^{\text {th }}$ observed regressor data for regressor variable $x_{j}$. Equation (5.32) is derived for a multiple regression model with $k$ regressor coefficients. Residuals scaled using the estimated variances given in equation (5.32) are referred to as the studentized residuals. The $i^{\text {th }}$ studentized residual, $e_{\text {studentized }(i)}$ can be calculated using the following relation

$$
e_{\text {studentized }(i)}=\frac{e_{i}}{\sqrt{M S S_{\text {error }}\left(1-h_{i i}\right)}}
$$

All the relations expressed in equations (5.32) through (5.35) can be found in References [55] and [59].

Figures 5.15 and 5.16 contain plots of studentized residuals as a function of the estimated equivalent mean and estimated equivalent standard deviation for both of the assumed initial crack configurations respectively. In both figures the data points are randomly scattered in a 
constant horizontal band and this is a good indication of constant error variance [60]. Plots that depict increasing or decreasing trend (e.g. funnel shape) clearly indicate non constant variance. All of the regression models of our study satisfy the error normality and the constant variance condition. 


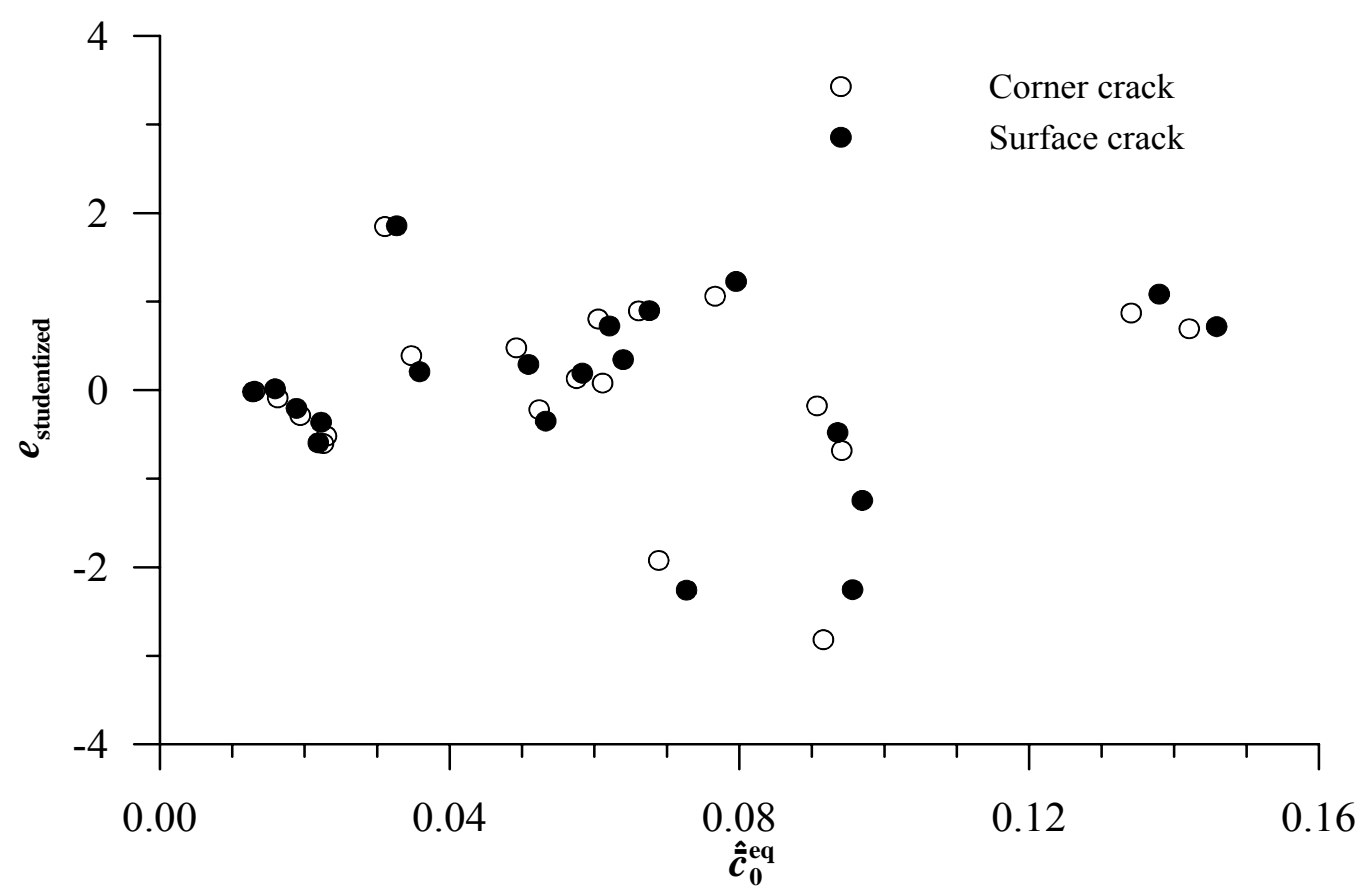

Figure 5.15 Studentized residual plot for estimated equivalent mean

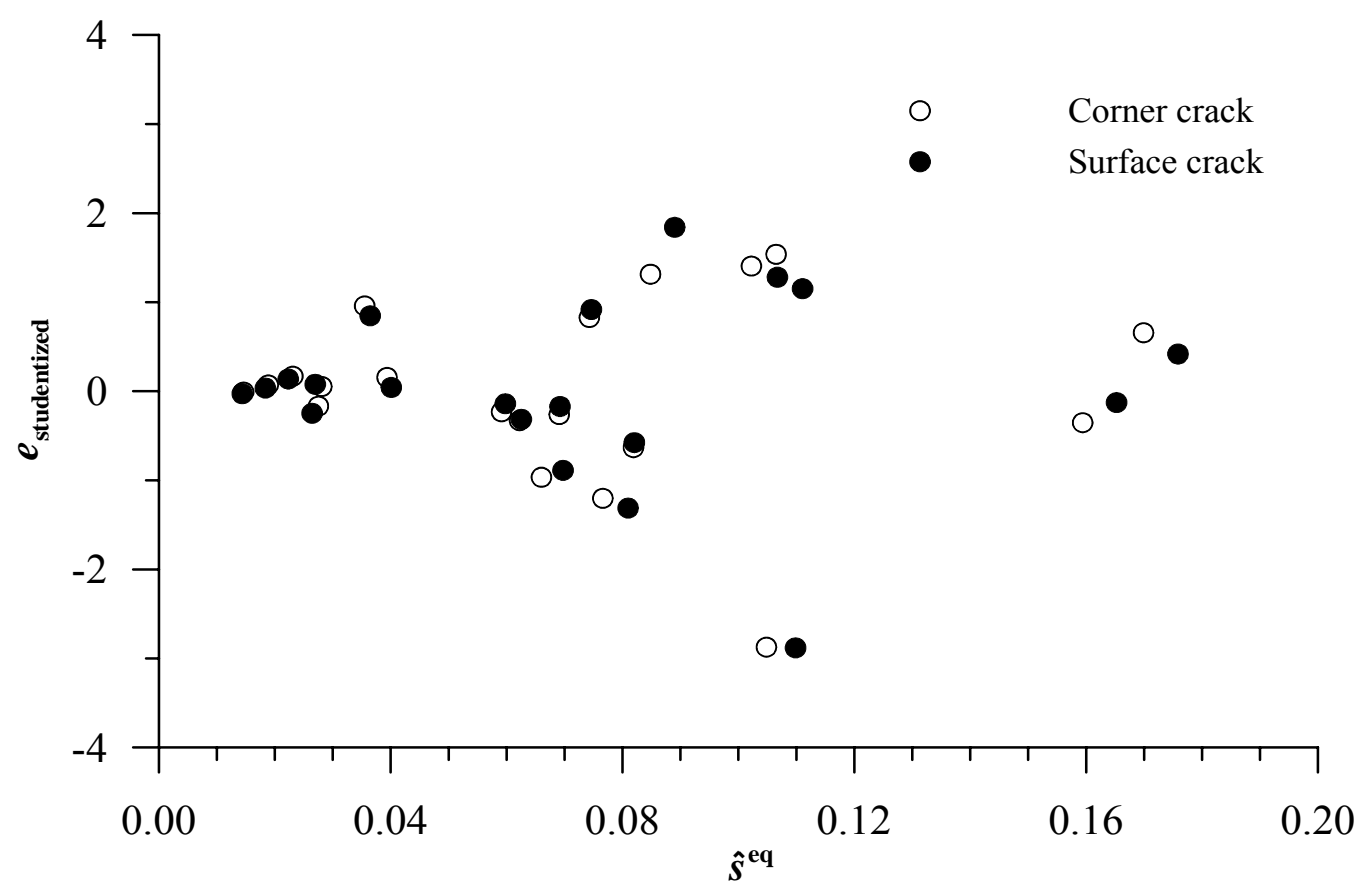

Figure 5.16 Studentized residual plot for estimated equivalent standard deviation 


\subsection{Hypothesis Testing of Regression Model Parameters}

Analyses of the variance of linear multiple regression models are usually presented along with hypothesis testing [56]. Hypothesis testing is a statistical procedure to determine the probability that a given hypothesis is true. The following two kinds of hypotheses testing were considered in this study and they are discussed in this section: a) test for significance of regression and $b$ ) test of individual regression coefficients.

Recall that the regression model developed in Section 5.4 was the generalized linear model, called the multiple linear regression model. The response variable in this model is a linear function of the regressor variables. Equation (5.11) (rewritten for convenience)

$$
\hat{y}=\hat{\beta}_{0}+\hat{\beta}_{1} x_{1}+\hat{\beta}_{2} x_{2}+\cdots+\hat{\beta}_{k} x_{k}
$$

is a general multiple linear regression model with $k$ regressor variables. The goal of the test for significance of regression is to determine if a statistically significant linear relationship exists between the response variable, $y$, and a subset of the regressor variables, $x_{1}, x_{2}, \ldots, x_{k}$ for the model of equation (5.11). A statistical result is called significant if it is unlikely to have occurred by chance. A statistically significant difference means that there is statistical evidence that there is a difference; it does not mean the difference is necessarily large, important, or significant in the usual sense of the word. The null hypothesis, $\mathrm{H}_{0}$, that all of the regressor coefficients equal to zero can be expressed as

$$
\mathrm{H}_{0}: \beta_{1}=\beta_{2}=\ldots=\beta_{k}=0
$$

while the alternate hypothesis, $\mathrm{H}_{\mathrm{a}}$, that at least one of the regressor coefficients does not equal to zero expressed as

$$
\mathrm{H}_{\mathrm{a}}: \beta_{i} \neq 0 \text { for at least one } i
$$


If the results of the appropriate decision-making procedures lead to the conclusion that the null hypothesis, $\mathrm{H}_{0}$, should be accepted then this would imply that none of the regressor variables, $x_{1}, x_{2}, \ldots, x_{k}$ contribute significantly to the model of equation (5.11). Conversely if the results of the decision-making procedures leads one to reject the $\mathrm{H}_{0}$, then this would imply that at least one of the model terms involving the regressor variables $x_{1}, x_{2}, \ldots, x_{k}$ contributes significantly to the estimated parameter, $\hat{y}$.

It was shown in Reference [58] that if the null hypothesis is true, then the mean regression sum of squares, $M S S_{\text {regression }}$, is distributed as a $\chi^{2}$-distribution (chi-square distribution) with $k$ degrees of freedom. Recall that $k$ is the number of regressor variables in the model. Similarly, it can also be shown that the $M S S_{\text {error }}$ has $\chi^{2}$-distribution with $n-k-1$ degrees of freedom, where $n$ is total number of observations (or sample size). The test procedure for the null hypothesis is to compute the test statistic, $F_{0}$, defined by the following expression

$$
F_{0}=\frac{M S S_{\text {regression }}}{M S S_{\text {error }}}
$$

where $M S S_{\text {regression }}$ and $M S S_{\text {error }}$ are given in equations (5.26) and (5.27) respectively. The null hypothesis, $\mathrm{H}_{0}$ is rejected if

$$
F_{0}>F_{\alpha, k, n-k-1}
$$

where $F_{\alpha, k, n-k-1}$, is a random variable that has an $F$-distribution with level of significance of $\alpha$ and $k$ and $n-k-1$ are the numerator and denominator degrees of freedom respectively. The $F$-distribution is a continuous probability distribution and is identified by three parameters: level of significance, numerator degrees of freedom, and denominator degrees of freedom. The 
level of significance is the probability that the null hypothesis will be rejected in error (assuming null hypothesis is true); a decision known as a Type I error [55-56]. The greater the level of significance, $\alpha$, the higher the probability of rejecting a true null hypothesis.

It is customary to report the observed level of significance, also called $P$-value of the test [55-56] rather than reporting the level of significance of a statistical test. The $P$-value, in actuality is the smallest level of significance, $\alpha$, that would lead to rejecting the null hypothesis. Having the smallest possible $P$-value would suggest the highest confidence in the rejection of the null hypothesis if it is not true. Many researchers prefer to make use of $P$-values when publishing the results of a statistical test of hypothesis. Instead of selecting a level of significance, $\alpha$, a priori and conducting a test as outlined in the previous paragraph, the researcher may compute the value of the appropriate test statistic and its associated $P$-value and leave to the reader of the report to judge the significance of the result. That is the reader must determine whether to reject the null hypothesis in favor of the alternative hypothesis, based on the reported $P$-value. Usually the null hypothesis will be rejected if the observed level of significance, $P$-value, is less than a fixed level of significance, $\alpha$. That is in mathematical terms,

$$
\text { If }(P \text { - value }<\alpha) \text { Then REJECT } \mathrm{H}_{0}
$$

The $P$-value associated with the test statistic, of $F_{0}$ is

$$
P \text { - value }=P\left(F_{k, n-k-1}>F_{0}\right)
$$

where $P\left(F_{k, n-k-1}>F_{0}\right)$ is the probability of $F_{k, n-k-1}$ being greater than $F_{0}$, where $F_{k, n-k-1}$ is a random variable that has an $F$-distribution with $k$ numerator degrees of freedom and $n-k-1$ denominator degrees of freedom. 
The second type of hypothesis testing considered was a test of individual regression coefficients. Testing the significance of the individual model parameters is useful in determining the contribution of each term to the overall response. For instance, by including additional variables or even deleting one or more variables the model effectiveness can be enhanced. In general, the addition of a variable to the regression model will always cause the regression sum of squares, $S S_{\text {regression }}$ to increase and the error sum of squares, $S S_{\text {error }}$ to decrease. The analyst must decide whether the increase in the regression sum of squares is sufficient to justify the addition of the variable. Moreover, the addition of an insignificant variable could actually increase the mean square error, thereby rendering the model less effective.

Consider the $i^{\text {th }}$ regression coefficient, $\beta_{i}$, of the model of equation (5.11), with, the associated null and alternative hypotheses,

$$
\mathrm{H}_{0}: \beta_{i}=0
$$

and

$$
\mathrm{H}_{\mathrm{a}}: \beta_{i} \neq 0
$$

This form of hypothesis testing must be carried out for each of the regression coefficients in the model, hence for the model represented by equation (5.11) the testing must be conducted for each regression coefficients $\beta_{1}, \beta_{2}, \ldots, \beta_{k}$. If the results of the decision-making procedures leads one to accept the null hypothesis, $\mathrm{H}_{0}$, then this would imply that regressor variable, $x_{i}$, does not contribute significantly to the model of equation (5.11). Hence, regressor variable $x_{i}$ can be deleted from the model with no penalty. Conversely if the results of the decision-making procedures leads one to reject the null hypothesis, $\mathrm{H}_{0}$, then this would imply that $x_{i}$ contributes 
significantly to the estimated variable, $\hat{y}$. The test statistic for the $i^{\text {th }}$ regression coefficient, $t_{0}$, is given by the following expression

$$
t_{0}=\frac{\hat{\beta}_{i}}{\sqrt{\hat{\sigma}^{2} C_{i i}}}
$$

from Reference [55] and [59]. In this equation $C_{i i}$ is the $i^{\text {th }}$ diagonal element of the square matrix, $\left(x^{T} \cdot x\right)^{-1}$ corresponding to $\hat{\beta}_{i}$, where $\boldsymbol{x}$ is as defined in equation (5.34), and $\hat{\sigma}^{2}$ is the estimated variance and is defined by the expression

$$
\hat{\sigma}^{2}=\frac{S S_{\text {error }}}{n-k}
$$

where $S S_{\text {error }}$ is the error sum of squares given in equation (5.24), $n$ is the total number of observations, and $k$ is the number of regressor variables. The null hypothesis, $\mathrm{H}_{0}$, is rejected if

$$
\left|t_{0}\right|>t_{\alpha / 2, n-k-1}
$$

where $t_{\alpha / 2, n-k-1}$, is the value of a $t$-distribution with level of significance of $\alpha / 2$ and $n-k-1$ degrees of freedom. The $P$-value associated with test statistic $t_{0}$ is

$$
P \text { - value }=2 P\left(t_{n-k-1}>\left|t_{0}\right|\right)
$$

where $P\left(t_{n-k-1}>\left|t_{0}\right|\right)$ is the probability of $t_{n-k-1}$ being greater than $\left|t_{0}\right|$, where $t_{n-k-1}$ is a random variable that has a $t$-distribution with $n-k-1$ degrees of freedom.

The multiple regression report from the STATGRAPHICS Plus software includes two other parameter values called multiple coefficient of determination, $R^{2}$, and adjusted multiple coefficient of determination, $R_{\text {adj }}^{2}$. The parameter $R^{2}$ is a measure of the reduction in the variability of the response variable as a consequence of using each of the regressor variables in the model. It is defined by the expression 


$$
R^{2}=1-\frac{S S_{\text {error }}}{S S_{\text {total }}}
$$

where $S S_{\text {error }}$ is given in equation (5.24) and $S S_{\text {total }}$ is given in equation (5.21). However, a large value of $R^{2}$ does not necessarily imply that the regression model is good, since adding a variable to the model will always increase $R^{2}$, regardless of whether the additional variable is statistically significant or not. Thus, it is possible for models with large values of $R^{2}$ to yield poor predictions of new observations, or estimates of the mean response.

Because $R^{2}$ always increases as terms are added to the model, some regression model builders prefer to use an adjusted multiple coefficient of determination, $R_{\mathrm{adj}}^{2}$. In general, the $R_{\mathrm{adj}}^{2}$ statistic will not always increase as variables are added to the model. $R_{\text {adj }}^{2}$ is defined as

$$
R_{\text {adj }}^{2}=1-\frac{n-1}{n-(k+1)}\left(1-R^{2}\right)
$$

where $n$ is the sample size, $k$ is the number of regressor variables and $R^{2}$ is given in equation (5.48). If unnecessary terms are added to the model, the value of $R_{\mathrm{adj}}^{2}$ will often decrease, thus if the $R^{2}$ and $R_{\text {adj }}^{2}$ values of a model differ dramatically, there is a good chance that nonsignificant terms have been included.

The multiple regression analysis and analysis of variance reports (ANOVA) for each of the regression equations (5.16) through (5.19) are presented in Appendix C. Both of these reports are made available by the STATGRAPHICS Plus software. The multiple regression analysis report presented the estimated coefficient, $\hat{\beta}_{i}$, the test statistic, $t_{0}$, as defined in equation (5.44) and the $P$-value associated with $t_{0}$ as defined in equation (5.47) for each regressor variable. The analysis of variance report presented the regression sum of squares, $S S_{\text {regression }}$, the 
error sum of squares, $S S_{\text {error }}$, the total sum of squares, $S S_{\text {total }}$, their associated degrees of freedom, the mean regression sum of squares, $M S S_{\text {regression }}$, the mean error sum of squares, $M S S_{\text {error }}$, the test statistic, $F_{0}$, as defined in equation (5.38), the $P$-value associated with $F_{0}$ as defined in equation (5.41), multiple coefficient of determination, $R^{2}$, and adjusted multiple coefficient of determination, $R_{\text {adj }}^{2}$. Table 5.1 contains some of the key information obtained from the multiple regression analysis report and the analysis of variance report. The first column represents the estimated variables of the regression. The second column contains $P$-values associated with test statistic, $F_{0}$ while the third and fourth columns contain $P$-values associated with statistic $t_{0}$ for each regressor variable $x_{1}$ and $x_{2}$ respectively. Each of equivalent standard deviation regression equation has one regressor variable while the equivalent mean regression equations have two regressor variables each. Columns five and six contain values of the $R^{2}$ and $R_{\text {adj }}^{2}$

In many engineering applications a level of significance of $\alpha=0.05$ is desirable. If the observed level of significance, $P$-value, is less than the chosen level of significance, $\alpha$, then there is sufficient confidence to reject the null hypothesis, $\mathrm{H}_{0}$. From column two of Table 5.1, all $P$-values associated with the test statistic, $F_{0}$, were less than an $\alpha$ value of 0.05 . This implies that at least one of the model terms involving $\left(x_{1}\right.$ or $\left.x_{2}\right)$ for the equivalent mean equations and $\left(x_{1}\right)$ for the equivalent standard deviation equations contributes significantly to the estimated parameter. Based on columns three and four of Table 5.1 all of the $P$-values associated with the test statistic, $t_{0}$, were also less than an $\alpha$ value of 0.05 . This implies that each regressor variable contributed significantly to the estimated parameter. Again note that no 
$P$-values were listed for the regressor variable $x_{2}$ for either $\hat{s}^{\text {eq }}$ term since neither is a function of that regressor variable. The $R^{2}$ and $R_{\text {adj }}^{2}$ values in columns five and six of the models are large (well above 95\%) and differ very slightly which is good indication that only significant terms were included in all regression models. All of the regression models of this study satisfied the hypotheses tests.

Table 5.1

Key parameters multiple regression analysis and analysis of variance reports

\begin{tabular}{|c|c|c|c|c|c|}
\hline$\hat{y}$ & $\begin{array}{c}P \text {-value } \\
(\text { Model })\end{array}$ & $\begin{array}{c}P \text {-value } \\
\left(x_{1}\right)\end{array}$ & $\begin{array}{c}P \text {-value } \\
\left(x_{2}\right)\end{array}$ & $R^{2}$ & $R_{\text {adj }}^{2}$ \\
\hline$\hat{\bar{c}}_{0, \text { corner }}^{\text {eq }}$ & 0.0000 & 0.0003 & 0.0349 & $99.3666 \%$ & $99.2921 \%$ \\
\hline$\hat{s}_{\text {corner }}^{\text {eq }}$ & 0.0000 & 0.0000 & - & $97.8302 \%$ & $97.7096 \%$ \\
\hline$\hat{\bar{c}}_{0, \text { surface }}^{\text {eq }}$ & 0.0000 & 0.0002 & 0.0347 & $99.6669 \%$ & $99.6277 \%$ \\
\hline$\hat{s}_{\text {surface }}^{\text {eq }}$ & 0.0000 & 0.0000 & - & $98.1982 \%$ & $98.0981 \%$ \\
\hline
\end{tabular}

\subsection{Estimated Values}

Now that the statistical models have been established, the estimated mean and standard deviation values of equivalent initial flaw sizes for the $K_{\mathrm{t}}=3$ and $K_{\mathrm{t}}=4$ specimens can be calculated. Rearranging the terms of equation (5.5), the estimated mean equations for both assumed initial crack configuration can be expressed as

$$
\hat{\bar{C}}_{0, \text { corner or surface }}=\operatorname{antilog}\left(-S_{\max } \hat{\bar{C}}_{0, \text { corner or surface }}^{\text {eq }}\right)
$$

where $\hat{\bar{C}}_{0, \text { corner }}^{\text {eq }}$ is given in equation (5.16) and $\hat{\bar{C}}_{0, \text { surface }}^{\text {eq }}$ is given in equation (5.18). Likewise, rearranging terms of equation (5.6) the estimated standard deviation equations for both assumed initial crack configuration can be expressed as 


$$
\hat{S}_{\text {corner or surface }}=\operatorname{antilog}\left(-S_{\max } \hat{S}_{\text {corner or surface }}^{\text {eq }}\right)
$$

where $\hat{s}_{\text {corner }}^{\text {eq }}$ is given in equation (5.17) and $\hat{s}_{\text {surface }}^{\text {eq }}$ is given in equation (5.19). By substituting the set of $S_{\text {mean }}, S_{\max }, K_{\mathrm{tg}}$, and $w_{\mathrm{n}}$ values the estimated mean and standard deviation of the equivalent initial flaw sizes were obtained. Tables 5.2 and 5.3 contain summaries of these values for the $K_{\mathrm{t}}=3$ and $K_{\mathrm{t}}=4$ specimen respectively. The sets of $S_{\text {mean }}, S_{\text {max }}, K_{\mathrm{tg}}$, and $w_{\mathrm{n}}$ values were chosen for which experimental tested data was available. Columns one and two of Tables 5.2 and 5.3 contain $S_{\text {mean }}$ and $S_{\max }$ values of the loading respectively. Column three and four contain the estimated mean and estimated standard deviation values of the equivalent initial flaw size for the assumed corner crack configuration. Similarly, columns five and six contain the estimated mean and estimated standard deviation values for the assumed surface crack configuration.

Table 5.2

Equivalent initial flaw size estimated mean and estimated standard deviation for $K_{\mathrm{t}}=3$ specimen $\left(K_{\mathrm{tg}}=5.165, w_{\mathrm{n}}=3.0480 \times 10^{-2} \mathrm{~m}\right)$

(Units for $S_{\text {mean }}$ and $S_{\max }$ are $M P a$ )

\begin{tabular}{|c|c|c|c|c|c|}
\hline$S_{\text {mean }}$ & $S_{\max }$ & $\hat{\bar{C}}_{0, \text { corner }}$ & $\hat{S}_{\text {corner }}$ & $\hat{\bar{C}}_{0 \text {,surface }}$ & $\hat{S}_{\text {corner }}$ \\
\hline 20.684 & 68.948 & $7.17558 \mathrm{E}-05$ & $1.46654 \mathrm{E}-05$ & $5.10609 \mathrm{E}-05$ & $9.77627 \mathrm{E}-06$ \\
\hline 20.684 & 48.263 & $4.46498 \mathrm{E}-05$ & $5.22688 \mathrm{E}-06$ & $3.66314 \mathrm{E}-05$ & $3.90235 \mathrm{E}-06$ \\
\hline 41.369 & 96.527 & $3.77582 \mathrm{E}-05$ & $1.20853 \mathrm{E}-05$ & $2.71166 \mathrm{E}-05$ & $7.57950 \mathrm{E}-06$ \\
\hline 41.369 & 62.053 & $2.07761 \mathrm{E}-05$ & $3.19381 \mathrm{E}-06$ & $1.78239 \mathrm{E}-05$ & $2.32452 \mathrm{E}-06$ \\
\hline
\end{tabular}




\section{Table 5.3}

Equivalent initial flaw size estimated mean and estimated standard deviation for $K_{\mathrm{t}}=4 \operatorname{specimen}\left(K_{\mathrm{tg}}=7.013, w_{\mathrm{n}}=3.0480 \times 10^{-2} \mathrm{~m}\right)$

(Units for $S_{\text {mean }}$ and $S_{\max }$ are $M P a$ )

\begin{tabular}{|c|c|c|c|c|c|}
\hline$S_{\text {mean }}$ & $S_{\max }$ & $\hat{\bar{c}}_{0, \text { corner }}$ & $\hat{s}_{\text {corner }}$ & $\hat{\bar{c}}_{0, \text { surface }}$ & $\hat{s}_{\text {corner }}$ \\
\hline 20.684 & 62.053 & $5.35515 \mathrm{E}-05$ & $1.58435 \mathrm{E}-05$ & $3.19266 \mathrm{E}-05$ & $8.97895 \mathrm{E}-06$ \\
\hline 20.684 & 37.921 & $2.74221 \mathrm{E}-05$ & $3.79277 \mathrm{E}-06$ & $1.99811 \mathrm{E}-05$ & $2.46855 \mathrm{E}-06$ \\
\hline 20.684 & 32.061 & $2.16976 \mathrm{E}-05$ & $2.24940 \mathrm{E}-06$ & $1.69942 \mathrm{E}-05$ & $1.54409 \mathrm{E}-06$ \\
\hline 41.369 & 75.842 & $2.32944 \mathrm{E}-05$ & $8.79670 \mathrm{E}-06$ & $1.46641 \mathrm{E}-05$ & $4.86260 \mathrm{E}-06$ \\
\hline 41.369 & 55.158 & $1.49801 \mathrm{E}-05$ & $3.36256 \mathrm{E}-06$ & $1.07575 \mathrm{E}-05$ & $2.04292 \mathrm{E}-06$ \\
\hline 41.369 & 51.711 & $1.36762 \mathrm{E}-05$ & $2.74608 \mathrm{E}-06$ & $1.00969 \mathrm{E}-05$ & $1.70267 \mathrm{E}-06$ \\
\hline
\end{tabular}

\subsection{Monte Carlo Simulation}

Monte Carlo simulation uses statistics to model a process and then estimate the likelihood of possible outcomes. It is widely used for simulating the behavior of various physical and mathematical systems. A set of random numbers is used iteratively as input to a deterministic model to create a set of output. Using a set of random input transforms the deterministic model into a stochastic model. Because of the repetition of algorithms and the large number of calculations involved, Monte Carlo simulation is suited to calculation using a computer.

Essentially, the application of the Monte Carlo method in the determination of estimated fatigue lives might be described in the following manner. With the estimated mean and standard deviation of the equivalent initial flaw size given in Tables 5.2 and 5.3, a set of random initial flaw sizes were generated from a normal distribution. These random initial flaw sizes were then used as input to FASTRAN-II code to generate estimated fatigue lives. The STATGRAPHICS 
Plus software was used to generate a set of random numbers from the normal distribution. A total of 75 random initial flaw sizes were generated for each case stress level case.

Before we proceed further it is essential to prove that the equivalent initial flaw sizes at each $S_{\max }$ level are normally distributed or approximately normally distributed for the smooth unnotched, open-hole, and $K_{\mathrm{t}}=5$ specimens. The following steps were involved in the construction of a normal probability plot for the equivalent initial flaw size, $c_{0, \text { corner or surface }}$. First the $i^{\text {th }}$ equivalent initial flaw size, $\left(c_{0, \text { corner or surface }}\right)_{i}$ at each $S_{\max }$ was ranked in the ascending order. Then the tail area of the $z$-distribution (standard normal distribution) corresponding to each ranked $i^{\text {th }}$ residual data, $A_{i}$, was determined from equation (5.30). Here $n$ is total number of equivalent initial flaw sizes at each $S_{\max }$ level. The expected value of the $i^{\text {th }}$ equivalent initial flaw size at each $S_{\max }$ level, $E\left(c_{0, \text { corner or surface }}\right)_{i}$ is expressed as

$$
E\left(c_{0, \text { corner or surface }}\right)_{i}=s_{\text {corner or surface }}\left[P\left(z \leq A_{i}\right)\right]
$$

where $P\left(z \leq A_{i}\right)$ is the probability of $z$ being less than or equal to $A_{i}$, where $z$ is a random variable that has a standard normal distribution and $s_{\text {conner or surface }}$ is the equivalent initial flaw size standard deviation and is defined in equation (5.4). The normal probability plot was constructed by plotting the ranked equivalent initial flaw size at each $S_{\max }$ level, $\left(c_{0, \text { corner or surface }}\right)_{i}$, on the vertical axis and the corresponding expected equivalent initial flaw size, $E\left(c_{0, \text { corner or surface }}\right)_{i}$ at the same stress level on the horizontal axis.

Figures 5.17 to 5.21 contain the equivalent initial flaw size normal probability plots for a few selected cases for the smooth unnotched, open-hole and $K_{\mathrm{t}}=5$ specimens. Each figure contains the probability plot for both assumed crack configuration types. In each case the plotted 
data points track the trend line relatively well thus validating the normality condition for the distribution of equivalent initial flaw size. The slope and $y$-intercept of the trend line were obtained from linear regression analysis; by regressing the ranked equivalent initial flaw size, $\left(c_{0, \text { corner or surface }}\right)$, on the corresponding expected equivalent initial flaw size, $E\left(c_{0 \text {,corner or surface }}\right)$.

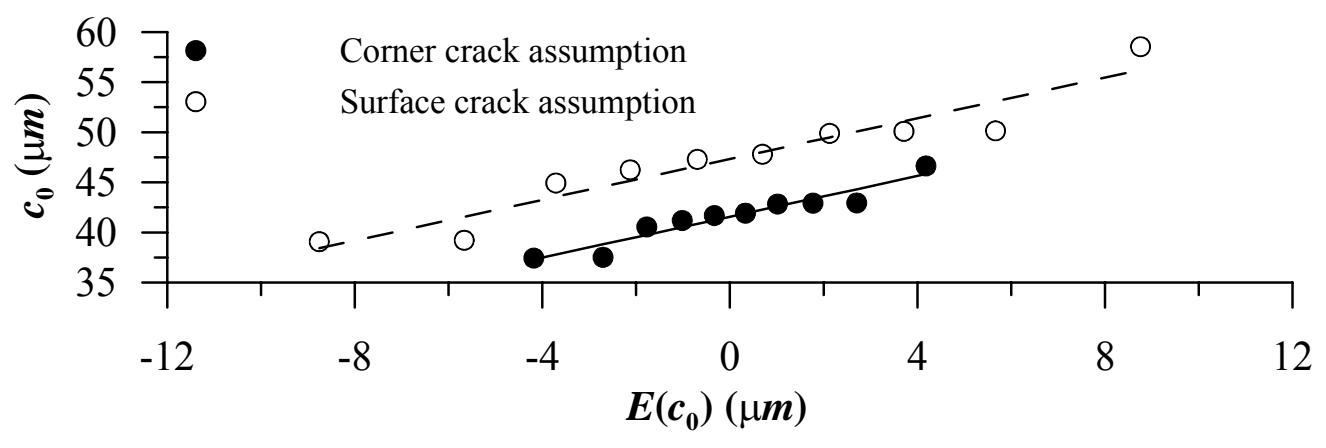

Figure 5.17 $c_{0}$ normal probability plot for the smooth unnotched specimen $\left(S_{\text {mean }}\right.$ of $144.684 \mathrm{MPa}$ and $S_{\max }$ of $210.290 \mathrm{MPa}$ )

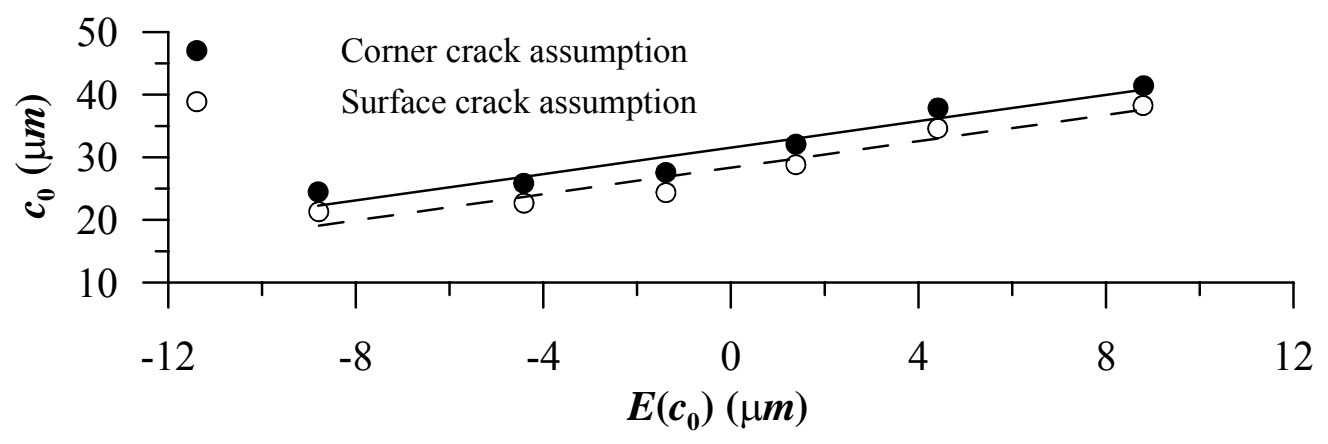

Figure 5.18 $c_{0}$ normal probability plot for the open-hole specimen $\left(S_{\text {mean }}\right.$ of $20.684 \mathrm{MPa}$ and $S_{\max }$ of $124.106 \mathrm{MPa}$ ) 


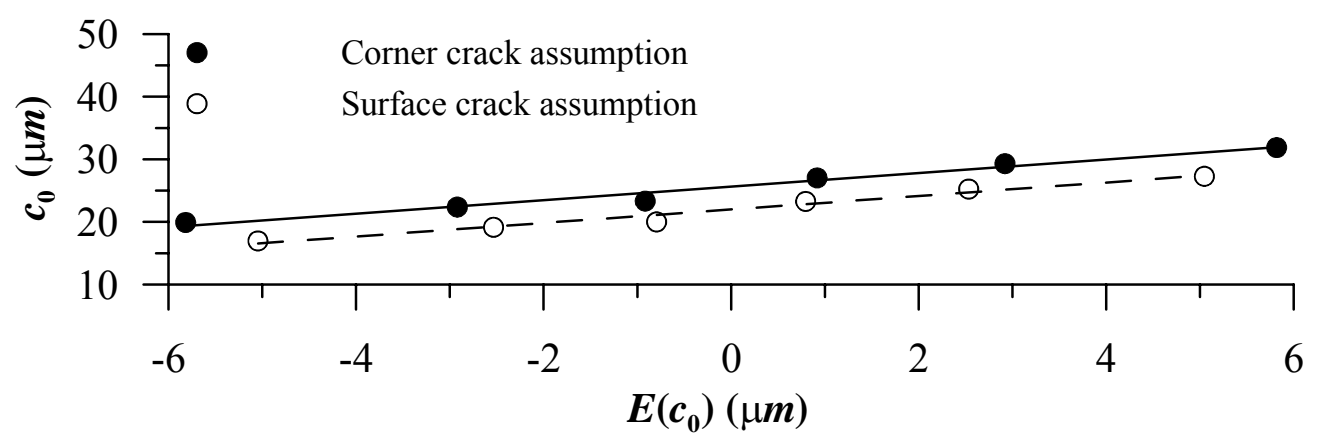

Figure 5.19 $c_{0}$ normal probability plot for open-hole specimen ( $S_{\text {mean }}$ of 41.369 MPa and $S_{\text {max }}$ of 79.290 MPa)

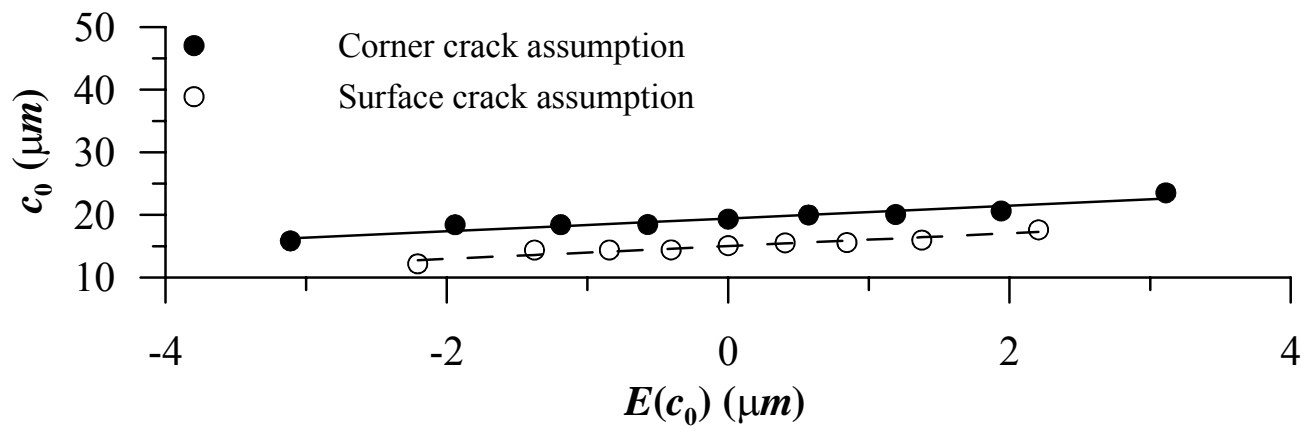

Figure 5.20 $c_{0}$ normal probability plot for the $K_{\mathrm{t}}=5$ specimen $\left(S_{\text {mean }}\right.$ of $20.684 \mathrm{MPa}$ and $S_{\text {max }}$ of $32.750 \mathrm{MPa}$ )

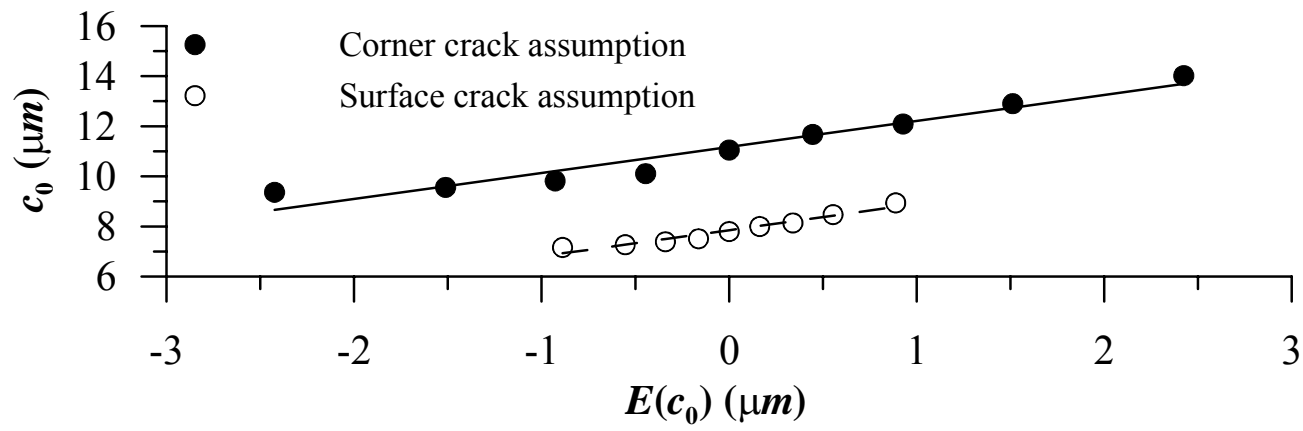

Figure 5.21 $c_{0}$ normal probability plot for the $K_{\mathrm{t}}=5$ specimen $\left(S_{\text {mean }}\right.$ of $41.369 \mathrm{MPa}$ and $S_{\text {max }}$ of $62.053 \mathrm{MPa}$ ) 
The resulting estimated fatigue lives are provided in Appendix D for the $K_{t}=3$ and $K_{\mathrm{t}}=4$ specimens. For some of the $K_{\mathrm{t}}=4$ specimens, when the randomly generated initial flaw sizes were small, the FASTRAN-II calculated crack growths were negligible and the corresponding lives exceeded 10 million cycles. In such cases the simulation was halted and the expected life data was not recorded for that particular initial flaw size. However, for the particular case of $S_{\text {mean }}$ of $41.368 \mathrm{MPa}$ and $S_{\max }$ of 51.711 $\mathrm{MPa}$, FASTRAN-II calculated negligible crack growth for all initial flaw sizes of the distributions for both assumed crack configurations.

The estimated fatigue life mean, $\bar{N}_{\text {corner or surface }}^{\mathrm{MC}}$, at each $S_{\max }$ was defined by the expression

$$
\bar{N}_{\text {corner or surface }}^{\mathrm{MC}}=\frac{\sum_{i=1}^{n}\left(N_{\text {corner or surface }}^{\mathrm{MC}}\right)_{i}}{n^{\mathrm{MC}}}
$$

where $\left(N_{\text {corner or surface }}^{\mathrm{MC}}\right)_{i}$ is the $i^{\text {th }}$ estimated fatigue life using either corner or surface assumed equivalent initial flaw size at each $S_{\max }$ level and $n^{\mathrm{MC}}$ is the sample size or total number of estimated fatigue lives in each $S_{\max }$ level. The estimated fatigue life standard deviation, $S_{\bar{N}, \text { corner or surface }}^{\mathrm{MC}}$, at each $S_{\max }$ was defined by the following expression

$$
S_{\bar{N}, \text { corner or surface }}=\sqrt{\frac{\sum_{i=1}^{n}\left[\left(N_{\text {corner or surface }}^{\mathrm{MC}}\right)_{i}-\bar{N}_{\text {corner or surface }}\right]^{2}}{n^{\mathrm{MC}}-1}}
$$

In probability and statistics, the standard deviation is usually expressed in dimensionless form known as the coefficient of variation. The coefficient of variation is a measure of dispersion of a probability distribution and is defined as the ratio of the standard deviation to the mean. The estimated fatigue life coefficient of variation is expressed as 


$$
c_{v, \text { corner or surface }}^{\mathrm{MC}}=\frac{S_{\bar{N}, \text { corner or surface }}^{\mathrm{MC}}}{\bar{N}_{\text {corner or surface }}^{\mathrm{MC}}}
$$

Tables 5.4 and 5.5 contain the means and coefficient of variations for the estimated fatigue life determined with the Monte Carlo simulation for the $K_{t}=3$ and the $K_{t}=4$ specimens. Columns one and two of the tables contain $S_{\text {mean }}$ and $S_{\max }$ values of the loading, columns three, four, and five contain the number of computed values, the means, and the coefficient of variations of the estimated fatigue life for the corner crack configuration, while columns six, seven, and eight contain the number of computed values, the means, and the coefficient of variations of the estimated fatigue life for the surface crack configuration. From Tables 5.4 and 5.5 it can be seen that the estimated fatigue life obtained from the corner crack assumption, $\bar{N}_{\text {corner }}^{\text {MC }}$ is less than the estimated fatigue life obtained from the surface crack assumption, $\bar{N}_{\text {surface }}^{\mathrm{MC}}$.

Table 5.4

Estimated fatigue life mean and coefficient of variation for $K_{\mathrm{t}}=3$ specimen (Units for $S_{\text {mean }}$ and $S_{\max }$ are $M P a$ )

\begin{tabular}{|c|c|c|c|c|c|c|c|}
\hline$S_{\text {mean }}$ & $S_{\max }$ & $n^{\mathrm{MC}}$ & $\bar{N}_{\text {corner }}^{\mathrm{MC}}$ & $c_{v, \text { corner }}^{\mathrm{MC}}$ & $n^{\mathrm{MC}}$ & $\bar{N}_{\text {surface }}^{\mathrm{MC}}$ & $c_{v, \text { surface }}^{\mathrm{MC}}$ \\
\hline 20.684 & 68.948 & 75 & 49215 & 0.0624 & 75 & 53525 & 0.0693 \\
\hline 20.684 & 48.263 & 75 & 199121 & 0.0345 & 75 & 230718 & 0.0507 \\
\hline 41.369 & 96.527 & 75 & 18125 & 0.2051 & 75 & 22921 & 0.2081 \\
\hline 41.369 & 62.053 & 74 & 398763 & 0.5918 & 75 & 496311 & 0.6209 \\
\hline
\end{tabular}




\section{Table 5.5}

Estimated fatigue life mean and coefficient of variation for $K_{\mathrm{t}}=4$ specimen (Units for $S_{\text {mean }}$ and $S_{\max }$ are $M P a$ )

\begin{tabular}{|c|c|c|c|c|c|c|c|}
\hline$S_{\text {mean }}$ & $S_{\max }$ & $n^{\mathrm{MC}}$ & $\bar{N}_{\text {corner }}^{\mathrm{MC}}$ & $c_{v, \text { corner }}^{\mathrm{MC}}$ & $n^{\mathrm{MC}}$ & $\bar{N}_{\text {surface }}^{\mathrm{MC}}$ & $c_{v, \text { surface }}^{\mathrm{MC}}$ \\
\hline 20.684 & 62.053 & 75 & 42129 & 0.0894 & 75 & 43366 & 0.0950 \\
\hline 20.684 & 37.921 & 75 & 347845 & 0.2615 & 74 & 413340 & 0.3551 \\
\hline 20.684 & 32.061 & 46 & 2603838 & 0.4175 & 25 & 3184441 & 0.6059 \\
\hline 41.369 & 75.842 & 74 & 32866 & 0.2013 & 75 & 35173 & 0.2466 \\
\hline 41.369 & 55.158 & 42 & 750795 & 0.5076 & 19 & 1170348 & 0.2817 \\
\hline 41.369 & 51.711 & - & - & - & - & - & - \\
\hline
\end{tabular}

\subsection{Hypothesis Testing of Simulation Results}

Once estimated fatigue life means and standard deviations were obtained, they were compared to the measured fatigue life means and standard deviations which were obtained through experimental testing to evaluate the validity of the theoretical prediction. The comparison was performed through statistical inference.

Inferential statistics comprises the use of statistics to make inferences concerning some unknown aspect of a population. It is distinguished from descriptive statistics. A statistical inference can be made in either of two ways - by estimating an unknown value of a parameter or making a decision about a hypothesized value of the parameter. Confidence intervals and hypothesis tests are related and can be used to make decisions about parameters. Before further testing was evaluated the means and standard deviations were determined for the measured fatigue life. The measured fatigue life mean, $\bar{N}$, at each $S_{\max }$ was determined from the following expression 


$$
\bar{N}=\frac{\sum_{i=1}^{n} N_{i}}{n}
$$

where $N_{i}$ is the $i^{\text {th }}$ measured fatigue life obtained at each $S_{\max }$ level and $n$ is the sample size. The measured fatigue lives were presented in the $S-N$ plots of in Section 2.2.

The measured fatigue life standard deviation, $S_{\bar{N}}$, at each $S_{\max }$ was defined by the following expression

$$
S_{\bar{N}}=\sqrt{\frac{\sum_{i=1}^{n}\left(N_{i}-\bar{N}\right)^{2}}{n-1}}
$$

The measured fatigue life coefficient of variation is expressed as

$$
C_{v}=\frac{S_{\bar{N}}}{\bar{N}}
$$

Tables 5.6 and 5.7 contain the mean and coefficient of variation values of the measured fatigue life for the $K_{\mathrm{t}}=3$ and $K_{\mathrm{t}}=4$ specimen respectively. Columns one and two contain the $S_{\text {mean }}$ and $S_{\max }$ values of the loading, column three is the number of specimens tested (or sample size), and columns four and five contain the means and standard deviations of the measured fatigue life. 


\section{Table 5.6}

Measured fatigue life mean and coefficient of variation for $K_{\mathrm{t}}=3$ specimen (Units for $S_{\text {mean }}$ and $S_{\max }$ are $M P a$ )

\begin{tabular}{|c|c|c|c|c|}
\hline$S_{\text {mean }}$ & $S_{\max }$ & $n$ & $\bar{N}$ & $c_{v}$ \\
\hline 20.684 & 68.948 & 5 & 69435 & 0.1293 \\
\hline 20.684 & 48.263 & 3 & 285749 & 0.1048 \\
\hline 41.369 & 96.527 & 5 & 28827 & 0.0821 \\
\hline 41.369 & 62.053 & 5 & 529815 & 0.4765 \\
\hline
\end{tabular}

Table 5.7

Measured fatigue life mean and coefficient of variation for $K_{t}=4$ specimen (Units for $S_{\text {mean }}$ and $S_{\text {max }}$ are $M P a$ )

\begin{tabular}{|c|c|c|c|c|}
\hline$S_{\text {mean }}$ & $S_{\max }$ & $n$ & $\bar{N}$ & $c_{v}$ \\
\hline 20.684 & 62.053 & 9 & 39572 & 0.1446 \\
\hline 20.684 & 37.921 & 9 & 381294 & 0.1159 \\
\hline 20.684 & 32.061 & 9 & 2686738 & 0.7540 \\
\hline 41.369 & 75.842 & 9 & 36862 & 0.1499 \\
\hline 41.369 & 55.158 & 9 & 462135 & 0.1403 \\
\hline 41.369 & 51.711 & 9 & 3533902 & 0.7840 \\
\hline
\end{tabular}

This section contains an explanation of the hypothesis testing of the simulation results. Hypothesis tests were conducted for the mean and standard deviation of the estimated fatigue life for both the $K_{\mathrm{t}}=3$ and $K_{\mathrm{t}}=4$ specimens at various stress levels.

The hypothesis test for comparing standard deviation values of the measured fatigue life and estimated fatigue life at each maximum stress level must be stated in terms of the ratio of two population variances. By definition the standard deviation is the square root variance. Let 
$\sigma^{2}$ be the measured fatigue life population variance and $\left(\sigma_{\text {corner or surface }}^{\mathrm{MC}}\right)^{2}$ be the estimated fatigue life population variance obtained from Monte Carlo simulation using either corner or surface crack assumption. The hypothesis for the testing ratio of two population variances can be expressed as

$$
\mathrm{H}_{0}: \frac{\sigma^{2}}{\left(\sigma_{\text {corner or surface }}\right)^{2}}=1
$$

and

$$
\mathrm{H}_{\mathrm{a}}: \frac{\sigma^{2}}{\left(\sigma_{\text {corner or surface }}\right)^{2}} \neq 1
$$

The expressions above simply states that if the decision-making procedures lead to a conclusion accepting the null hypothesis, $\mathrm{H}_{0}$, then the variance of the measured fatigue life is equal to the variance of the estimated fatigue life. Conversely if the decision-making procedures lead to the rejection of the $\mathrm{H}_{0}$ then this would imply that the variance of the two populations is not equal.

The test statistic, $F_{0}$, taken from Reference [56] at each $S_{\max }$ level is given by

$$
F_{0}=\left\{\begin{array}{l}
\frac{\left(s_{\bar{N}}\right)^{2}}{\left(s_{\bar{N}, \text { corner or surface }}^{\mathrm{MC}}\right)^{2}} \text { when }\left(s_{\bar{N}}\right)^{2}>\left(s_{\bar{N}, \text { corner or surface }}\right)^{2} \\
\frac{\left(s_{\bar{N}, \text { corner or surface }}\right)^{2}}{\left(s_{\bar{N}}\right)^{2}} \text { when }\left(s_{\bar{N}, \text { corner or surface }}^{\mathrm{MC}}\right)^{2}>\left(s_{\bar{N}}\right)^{2}
\end{array}\right.
$$

where $s_{\bar{N}}$ was defined in equation (5.57) and $s_{\bar{N}, \text { corner or surface }}^{\mathrm{MC}}$ was defined by equation (5.54). The assumptions made in equation (5.61) are that (1) both of the populations from which the samples are selected have relative frequency distributions that are approximately normal and (2) the 
random samples are selected in an independent manner from the two populations. The null hypothesis, $\mathrm{H}_{0}$ is rejected if

$$
F_{0}>F_{\alpha / 2, v_{1}, v_{2}}
$$

where $F_{\alpha / 2, v_{1}, v_{2}}$, is the value of the $F$-distribution with a level of significance of $\alpha / 2$ and $v_{1}$ and $v_{2}$ are the numerator and denominator degrees of freedom respectively. The $P$-value associated with the test statistic, of $F_{0}$ is

$$
P \text { - value }=2 P\left(F_{v_{1}, v_{2}}>F_{0}\right)
$$

where $P\left(F_{v_{1}, v_{2}}>F_{0}\right)$ is the probability of $F_{v_{1}, v_{2}}$ being greater than $F_{0}$ where $F_{v_{1}, v_{2}}$ is a random variable that has an $F$-distribution with $v_{1}$ numerator degrees of freedom and $v_{2}$ denominator degrees of freedom. The degrees of freedom associated with measured fatigue life is $n-1$, and the degrees of freedom associated with estimated fatigue life is $n^{\mathrm{MC}}-1$.

Tables 5.8 and 5.9 contain the $P$-values of the hypotheses test results for differences between the measured population and the estimated population for the $K_{\mathrm{t}}=3$ and $K_{\mathrm{t}}=4$ specimens respectively. Column one contains the $S_{\text {mean }}$ and column two contains the $S_{\max }$ values of the loading, while column three contains the $P$-values associated with $F_{0}$ for the corner crack assumption and column four contains the $P$-values associated with $F_{0}$ for the surface crack assumption. 


\section{Table 5.8}

$P$-values for variance hypothesis test results for $K_{t}=3$ specimen (Units for $S_{\text {mean }}$ and $S_{\max }$ are $M P a$ )

\begin{tabular}{|c|c|c|c|}
\hline$S_{\text {mean }}$ & $S_{\max }$ & $\begin{array}{c}P \text {-value } \\
F_{0, \text { corner }}\end{array}$ & $\begin{array}{c}P \text {-value } \\
F_{0, \text { surface }}\end{array}$ \\
\hline 20.684 & 68.948 & 0.000 & 0.001 \\
\hline 20.684 & 48.263 & 0.000 & 0.005 \\
\hline 41.369 & 96.527 & 0.391 & 0.177 \\
\hline 41.369 & 62.053 & 0.685 & 0.772 \\
\hline
\end{tabular}

Table 5.9

$P$-values for variance hypothesis test results for $K_{t}=4$ specimen (Units for $S_{\text {mean }}$ and $S_{\max }$ are $M P a$ )

\begin{tabular}{|c|c|c|c|}
\hline$S_{\text {mean }}$ & $S_{\text {max }}$ & $\begin{array}{c}P \text {-value } \\
F_{0, \text { corner }}\end{array}$ & $\begin{array}{c}P \text {-value } \\
F_{0, \text { surface }}\end{array}$ \\
\hline 20.684 & 62.053 & 0.226 & 0.135 \\
\hline 20.684 & 37.921 & 0.197 & 0.001 \\
\hline 20.684 & 32.061 & 0.007 & 0.792 \\
\hline 41.369 & 75.842 & 0.614 & 0.172 \\
\hline 41.369 & 55.158 & 0.000 & 0.000 \\
\hline 41.369 & 51.711 & - & - \\
\hline
\end{tabular}

In Table 5.8, the $P$-values associated with the variance (standard deviation) hypothesis test for $K_{\mathrm{t}}=3$ specimens for an $S_{\text {mean }}$ of $20.684 \mathrm{MPa}$ and for both an $S_{\max }$ of 68.948 and of 48.362 $\mathrm{MPa}$ were all less than 0.05 for both assumed initial crack configurations, hence the null hypothesis was rejected for each case. Rejection of the null hypothesis indicates that the measured fatigue life population variance was significantly different from the estimated fatigue life population variance obtained with a Monte Carlo simulation. Conversely, for the case of an 
$S_{\text {mean }}$ of $41.369 \mathrm{MPa}$ and an $S_{\max }$ of 96.527 and of $62.053 \mathrm{MPa}$ the $P$-values for both assumed initial cracks were greater than 0.05 therefore the null hypothesis was accepted for each case indicating that the estimated fatigue life population variance was similar to the measured fatigue life population variance.

The $P$-values associated with the variance hypothesis test for $K_{\mathrm{t}}=4$ specimens were presented in Table 5.9. The null hypothesis was accepted for the fatigue loading of $S_{\text {mean }}$ of 20.684 MPa and $S_{\max }$ of $62.053 \mathrm{MPa}$ for both assumed initial crack configurations since the $P$-values of both cases were greater than 0.05 . However, for the cases of $S_{\text {mean }}$ of $20.684 \mathrm{MPa}$ and $S_{\max }$ of $37.921 \mathrm{MPa}$ the null hypothesis was accepted for the assumed initial corner crack configuration and was rejected for the assumed initial surface crack configuration. In the third case with an $S_{\text {mean }}$ of $20.684 \mathrm{MPa}$ and a $S_{\text {max }}$ of $32.061 \mathrm{MPa}$ the null hypothesis was rejected for the assumed corner crack configuration and accepted for the assumed surface crack configuration. For the specimens subjected to a fatigue load of $S_{\text {mean }}$ of $41.369 \mathrm{MPa}$ and $S_{\max }$ of $75.842 \mathrm{MPa}$ the null hypothesis was accepted for both assumed initial crack configurations. On the other hand the specimen loaded with an $S_{\text {mean }}$ of $41.369 \mathrm{MPa}$ and an $S_{\text {max }}$ of 55.158 $M P a$ resulted in a $P$-value less than 0.05 which supported the rejection of the null hypothesis for both assumed initial crack configurations. The population variance hypothesis test could not be performed for the load case of $S_{\text {mean }}$ of $41.369 \mathrm{MPa}$ and $S_{\text {max }}$ of $51.711 \mathrm{MPa}$ because the FASTRAN-II output exceeded more than 10 million cycles.

The hypothesis test for comparing mean values of the measured fatigue life and estimated fatigue life at each maximum stress level must be stated in terms of population parameters. Let $\mu$ be the measured fatigue life population mean and $\mu_{\text {corner or surface }}^{\mathrm{MC}}$ be the estimated fatigue life 
population mean obtained from Monte Carlo simulation using either corner or surface crack assumption. The hypothesis for testing differences between two populations is expressed as

$$
\mathrm{H}_{0}:\left(\mu-\mu_{\text {corner or surface }}^{\mathrm{MC}}\right)=0
$$

and

$$
\mathrm{H}_{\mathrm{a}}:\left(\mu-\mu_{\text {corner or surface }}^{\mathrm{MC}}\right) \neq 0
$$

The above expression states that if the decision-making procedures lead to an acceptance of the null hypothesis, $\mathrm{H}_{0}$, then this would imply that the measured mean life is equal to the estimated mean life. Conversely, if the decision-making procedures leads to the rejection of the $\mathrm{H}_{0}$ then this would imply that the two mean values are not equal.

When the variance of the two populations are equal, i.e., $\sigma^{2}=\left(\sigma_{\text {corner or surface }}^{\mathrm{MC}}\right)^{2}$, the test statistic, $t_{0}$, taken from Reference [56] at each $S_{\text {max }}$ level is given by

$$
t_{0}=\frac{\bar{N}-\bar{N}_{\text {corner or surface }}^{\mathrm{MC}}}{\sqrt{s_{p}^{2}\left(\frac{1}{n}+\frac{1}{n^{\mathrm{MC}}}\right)}}
$$

where $s_{p}^{2}$ is defined as,

$$
s_{p}^{2} \equiv \frac{(n-1)\left(s_{\bar{N}}\right)^{2}+\left(n^{\mathrm{MC}}-1\right)\left(s_{\bar{N}, \text { corner or surface }}\right)^{2}}{n+n^{\mathrm{MC}}-2}
$$

$\bar{N}$ is defined by equation (5.56), $\bar{N}_{\text {corner or surface }}^{\mathrm{MC}}$ is defined by equation (5.53), $n$ is the sample size of the measured fatigue life, $n^{\mathrm{MC}}$ is the sample size of the estimated fatigue life, $s_{\bar{N}}$ is defined by equation (5.57), and $s_{\bar{N}, \text { corner or surface }}^{\mathrm{MC}}$ is defined by equation (5.54). The assumptions made in equation (5.66), from [Reference 56], are (1) the populations from which both samples are selected have approximately normal relative frequency distributions, (2) the variances of the two 
populations are equal, and (3) the random samples are selected in an independent manner from each of the two populations.

However for the case where the variance of the two populations are not equal, i.e., $\sigma^{2} \neq\left(\sigma_{\text {corner or surface }}^{\mathrm{MC}}\right)^{2}$, the test statistic, $t_{0}$, taken from Reference [56] at each $S_{\max }$ level is given by

$$
t_{0}=\frac{\bar{N}-\bar{N}_{\text {corner or surface }}^{\mathrm{MC}}}{\sqrt{\frac{\left(s_{\bar{N}}\right)^{2}}{n}+\frac{\left(s_{\bar{N}, \text { corner or surface }}^{\mathrm{MC}}\right)^{2}}{n^{\mathrm{MC}}}}}
$$

and the degrees of freedom, $v$ is defined as,

$$
v=\frac{\left[\frac{\left(s_{\bar{N}}\right)^{2}}{n}+\frac{\left(s_{\bar{N}, \text { corner or surface }}^{\mathrm{MC}}\right)^{2}}{n^{\mathrm{MC}}}\right]^{2}}{\frac{\left[\frac{\left(s_{\bar{N}}\right)^{2}}{n}\right]^{2}}{n-1}+\frac{\left[\frac{\left(s_{\bar{N}, \text { corner or surface }}\right)^{2}}{n^{\mathrm{MC}}}\right]^{2}}{n^{\mathrm{MC}}-1}}
$$

Note: The value of $v$ will generally not be an integer and therefore has to be rounded down to the nearest integer.

In equation (5.68) $\bar{N}$ is defined by equation (5.56), $\bar{N}_{\text {corner or surface }}^{\text {MC }}$ is defined by equation (5.53), $n$ is the sample size of the measured fatigue life, $n^{\mathrm{MC}}$ is the sample size of the estimated fatigue life, $s_{\bar{N}}$ is defined by equation (5.57), and $s_{\bar{N} \text {,corner or surface }}^{\mathrm{MC}}$ is defined by equation (5.54). The assumptions made in equation (5.68), from [Reference 56], are (1) the populations from which both samples are selected have approximately normal relative frequency distributions, (2) the variances of the two populations are not equal, and (3) the random samples are selected in an independent manner from each of the two populations.

The null hypothesis, $\mathrm{H}_{0}$, is rejected if the magnitude of the test statistic satisfies 


$$
\left|t_{0}\right|>t_{\alpha / 2, n+n^{\mathrm{MC}}-2}
$$

where $t_{\alpha / 2, n+n^{\mathrm{MC}}-2}$, is based on a $t$-distribution with level of significance of $\alpha / 2$ and $n+n^{\mathrm{MC}}-2$ degrees of freedom. The $P$-value associated with test statistic $t_{0}$ is

$$
P \text { - value }=2 P\left(t_{n+n^{\mathrm{MC}}-2}>\left|t_{0}\right|\right)
$$

Equation (5.71) states that the $P$-value is equal to twice the probability that $t_{n+n^{\mathrm{MC}}-2}$ is greater than $\left|t_{0}\right|$ where $t_{n+n^{\mathrm{MC}}-2}$ is a random variable that has a $t$-distribution with $n+n^{\mathrm{MC}}-2$ degrees of freedom. Tables 5.10 and 5.11 contain the resulting $P$-values of the hypotheses test for differences between two populations for the $K_{\mathrm{t}}=3$ and $K_{\mathrm{t}}=4$ specimens respectively. Columns one and two contain the $S_{\text {mean }}$ and $S_{\max }$ values of the loading respectively. Columns three and four contain the $P$-values associated with the magnitude of the test statistics, $\left|t_{0}\right|$, for the assumption of an initial corner crack and surface crack respectively.

Table 5.10

$P$-values for mean hypothesis test result for $K_{\mathrm{t}}=3$ specimen (Units for $S_{\text {mean }}$ and $S_{\text {max }}$ are $M P a$ )

\begin{tabular}{|c|c|c|c|}
\hline$S_{\text {mean }}$ & $S_{\max }$ & $\begin{array}{c}P \text {-value } \\
\left|t_{0, \text { corner }}\right|\end{array}$ & $\begin{array}{c}P \text {-value } \\
\left|t_{0, \text { surface }}\right|\end{array}$ \\
\hline 20.684 & 68.948 & 0.007 & 0.017 \\
\hline 20.684 & 48.263 & 0.038 & 0.087 \\
\hline 41.369 & 96.527 & 0.000 & 0.008 \\
\hline 41.369 & 62.053 & 0.235 & 0.813 \\
\hline
\end{tabular}




\section{Table 5.11}

$P$-values for mean hypothesis test result for $K_{\mathrm{t}}=4$ specimen (Units for $S_{\text {mean }}$ and $S_{\text {max }}$ are $M P a$ )

\begin{tabular}{|c|c|c|c|}
\hline$S_{\text {mean }}$ & $S_{\max }$ & $\begin{array}{c}P \text {-value } \\
\left|t_{0, \text { corner }}\right|\end{array}$ & $\begin{array}{c}P \text {-value } \\
\left|t_{0, \text { surface }}\right|\end{array}$ \\
\hline 20.684 & 62.053 & 0.114 & 0.014 \\
\hline 20.684 & 37.921 & 0.153 & 0.159 \\
\hline 20.684 & 32.061 & 0.905 & 0.517 \\
\hline 41.369 & 75.842 & 0.086 & 0.571 \\
\hline 41.369 & 55.158 & 0.000 & 0.000 \\
\hline 41.369 & 51.711 & - & - \\
\hline
\end{tabular}

The mean hypotheses test results for the $K_{t}=3$ specimen were presented in Table 5.10 while the mean hypothesis test results for the $K_{t}=4$ specimens were presented in Table 5.11. A level of significance, $\alpha$ of 0.05 was used for the criteria of accepting or rejecting the null hypothesis, $\mathrm{H}_{0}$. That is, if the $P$-value of the hypothesis test was less than 0.05 , the null hypothesis was rejected.

The $P$-values, reported in Table 5.10, associated with the mean hypothesis test for $K_{\mathrm{t}}=3$ specimens for an $S_{\text {mean }}$ of $20.684 \mathrm{MPa}$ and an $S_{\text {max }}$ of 68.948 and for an $S_{\text {mean }}$ of 41.369 $M P a$ and an $S_{\max }$ of $96.527 \mathrm{MPa}$, the $P$-values were all less than 0.05 for both assumed initial crack configurations hence resulting in the rejection of the null hypothesis. Rejection of the null implies that the measured fatigue life population mean is significantly different than the estimated fatigue life population mean obtained through a Monte Carlo simulation. However for the case an $S_{\text {mean }}$ of $20.684 \mathrm{MPa}$ and an $S_{\text {max }}$ of $48.263 \mathrm{MPa}$, the null hypothesis was rejected for corner crack case and was accepted for the surface crack case. For the case of an $S_{\text {mean }}$ of 
41.369 $\mathrm{MPa}$ and an $S_{\max }$ of $62.053 \mathrm{MPa}$ the $P$-values for both assumed initial crack types were greater than 0.05 therefore indicating that the estimated fatigue life population mean were similar to the measured fatigue life population mean.

In Table 5.11, the $P$-values associated with the hypothesis test of the mean for the $K_{\mathrm{t}}=4$ specimens for an $S_{\text {mean }}$ of $20.684 \mathrm{MPa}$ and an $S_{\max }$ of $62.053 \mathrm{MPa}$ were 0.114 for the assumed initial corner crack and 0.014 for the assumed initial surface crack. Therefore, the null hypothesis was accepted for the former case and rejected for the latter case.

As for $S_{\text {mean }}$ of 20.684 MPa and $S_{\text {max }}$ of 37.921 and $32.061 \mathrm{MPa}$ and $S_{\text {mean }}$ of 41.369 $M P a$ and $S_{\max }$ of $75.842 \mathrm{MPa}$ all the $P$-values were greater than 0.05 indicating that the estimated fatigue life population mean obtained with a Monte Carlo simulation were similar to the measured predicted life population mean. However, for an $S_{\text {mean }}$ of $41.369 \mathrm{MPa}$ and an $S_{\max }$ of $55.158 \mathrm{MPa}$ the $P$-values were less than 0.05 for both assumed initial configurations resulting in a rejection of the null hypothesis for both cases. For the case of an $S_{\text {mean }}$ of 41.369 $M P a$ and an $S_{\max }$ of $55.158 \mathrm{MPa}$ the initial flaw sizes were so small that the FASTRAN-II predicted lives exceeded 10 million cycles. 


\section{CHAPTER 6}

\section{DISCUSSION OF RESULTS}

Summaries of the hypothesis test results for the $K_{\mathrm{t}}=3$ and $K_{\mathrm{t}}=4$ specimens are presented in Tables 6.1 and 6.2 respectively. Columns one and two contain the $S_{\text {mean }}$ and $S_{\max }$ values of the fatigue associated with each $S-N$ test. Columns three and four contain the results of the null hypothesis tests results for the assumed initial corner crack and the assumed initial surface crack respectively. If both the mean and the variance of the hypothesis tests resulted in accepting the null hypotheses for a particular combination of mean stress, maximum stress, and form of initial crack then the null hypothesis was accepted, indicating that the estimated fatigue lives from Monte Carlo simulation were similar to the measured fatigue lives of the laboratory tests at level of significance, $\alpha$ of 0.05 . However, if either of the null hypothesis was rejected for a particular combination of mean stress, maximum stress, and form of initial crack, then the estimated fatigue lives from Monte Carlo simulation were significantly different as compared to the measured fatigue lives of the laboratory tests at $\alpha$ of 0.05 .

Based on these criteria, the null hypothesis was rejected for the $K_{\mathrm{t}}=3$ specimens specified by $S_{\text {mean }}$ of $20.684 \mathrm{MPa}$ for both maximum load cases and both crack configuration types. Likewise, the null hypothesis was also rejected for the fatigue load case of an $S_{\text {mean }}$ of 41.369 $\mathrm{MPa}$ and an $S_{\max }$ of $96.527 \mathrm{MPa}$ for both initial crack configurations. However, the null hypothesis was accepted for the fatigue loading defined by an $S_{\text {mean }}$ of $41.369 \mathrm{MPa}$ and an $S_{\max }$ of $62.053 \mathrm{MPa}$ for both assumed initial crack configurations. 
Using the same criteria for the $K_{\mathrm{t}}=4$ specimens, for the loading specified by $S_{\text {mean }}$ of 20.684 MPa and for the $S_{\max }$ of 62.053 and $37.921 \mathrm{MPa}$ the null hypothesis was accepted for the assumed initial corner crack configuration but was rejected for the assumed surface crack configuration. On the other hand, for the case of $S_{\text {mean }}$ of $20.684 \mathrm{MPa}$ and $S_{\max }$ of 32.061 $M P a$ the null hypothesis was rejected for assumed corner crack configuration but was accepted for the assumed surface crack configuration. For the specimens subjected to a fatigue load of $S_{\text {mean }}$ of $41.369 \mathrm{MPa}$ and $S_{\max }$ of $75.842 \mathrm{MPa}$ the null hypothesis was accepted for both assumed initial crack configurations however for the specimens subjected to fatigue load of $S_{\text {mean }}$ of $41.369 \mathrm{MPa}$ and $S_{\max }$ of $55.158 \mathrm{MPa}$ the null hypothesis was rejected for both assumed initial crack configurations.

Table 6.1

Summary of hypothesis test results for $K_{\mathrm{t}}=3$ specimen (Units for $S_{\text {mean }}$ and $S_{\max }$ are $M P a$ )

\begin{tabular}{|c|c|c|c|}
\hline$S_{\text {mean }}$ & $S_{\text {max }}$ & $\begin{array}{c}\mathrm{H}_{0, \text { corner }} \\
\text { (mean and variance) }\end{array}$ & $\begin{array}{c}\mathrm{H}_{0, \text { surface }} \\
\text { (mean and variance) }\end{array}$ \\
\hline 20.684 & 68.948 & Rejected & Rejected \\
\hline 20.684 & 48.263 & Rejected & Rejected \\
\hline 41.369 & 96.527 & Rejected & Rejected \\
\hline 41.369 & 62.053 & Accepted & Accepted \\
\hline
\end{tabular}




\section{Table 6.2}

Summary of hypothesis test results for $K_{\mathrm{t}}=4$ specimen (Units for $S_{\text {mean }}$ and $S_{\max }$ are $M P a$ )

\begin{tabular}{|c|c|c|c|}
\hline$S_{\text {mean }}$ & $S_{\text {max }}$ & $\begin{array}{c}\mathrm{H}_{0, \text { corner }} \\
\text { (mean and variance) }\end{array}$ & $\begin{array}{c}\mathrm{H}_{0, \text { surface }} \\
\text { (mean and variance) }\end{array}$ \\
\hline 20.684 & 62.053 & Accepted & Rejected \\
\hline 20.684 & 37.921 & Accepted & Rejected \\
\hline 20.684 & 32.061 & Rejected & Accepted \\
\hline 41.369 & 75.842 & Accepted & Accepted \\
\hline 41.369 & 55.158 & Rejected & Rejected \\
\hline
\end{tabular}

Based upon the hypothesis results presented in Tables 6.1 and 6.2 the proposed statistical model of the distribution of the equivalent initial crack size seems to better estimate lives for the case of the $K_{\mathrm{t}}=4$ specimens than for the $K_{\mathrm{t}}=3$ specimens at $\alpha$ of 0.05 . A possible reason for this could be that the numbers of samples used to collect experimental fatigue life data were between three to five for each of the $K_{\mathrm{t}}=3$ specimens stress level, while nine coupons were tested for each of the $K_{\mathrm{t}}=4$ specimens combination of mean and maximum stress. Data collected using more specimens generally reduces the variability of the test results.

While the proposed methodology was not able to estimate the fatigue lives for all stress levels and specimen types, the general idea is worth exploring further. There are some rational explanations as to why the methodology did not function as anticipated.

One explanation was a possible manufacturing flaw in the $K_{t}=5$ specimen. In the original design of these specimens the $\mathrm{V}$-notch was designed to join the circular notch at a point of tangency. The finite element model was constructed according to this original design, therefore, the stress concentration factor and stress intensity factors were evaluated using finite 
element model as a basis. However, flawed specimens were manufactured and the measured data were collected with flawed specimens.

Another possible explanation is also related to manufacturing. The manufacturing process used to machine the notches for both the $K_{t}=4$ and $K_{t}=5$ specimens were rather different than the one used to machine the $K_{\mathrm{t}}=3$ specimens. For both the $K_{\mathrm{t}}=4$ and $K_{\mathrm{t}}=5$ specimens two operations were involved in the machining process. First the hole was drilled using a drilling operation and the rest of the notch was milled out in a separate operation. An error in the milling operation led to a manufacturing flaw for the $K_{\mathrm{t}}=5$ specimens as explained in the previous paragraph. As for the $K_{\mathrm{t}}=3$ specimens the notches were machined out in a single drilling operation.

Based on observed experimental results reported in the Reference [33] and also through personal communication with Dr. Jim Newman, in most cases cracks tend to originate as a corner flaw in open-hole specimens and as surface cracks in notched and unnotched specimens. Based on these reported observations, perhaps the regression model should be modified so that flaw sizes for open-hole specimens are based on an initial corner flaw, and flaw sizes for all other specimens have an assumed initial surface flaw. 


\section{CHAPTER 7}

\section{CONCLUSIONS AND RECOMMENDATIONS}

In this investigation a statistically based analytical method was proposed that could generate a distribution of estimated fatigue lives for 2024-T3 aluminum alloy specimens with various stress concentration factors subjected to constant amplitude fatigue loads. The statistical method was based on a distribution of equivalent initial flaw size concept. The influence of the initial flaw, either corner crack or surface crack, was also investigated in this study. The equivalent initial flaw sizes were calculated using FASTRAN-II crack growth software using experimentally measured fatigue lives. The stress intensity factor for each specimen was required for input to the FASTRAN-II code. These stress intensity factors were computed using finite element methods for those specimens which were not available in the FASTRAN-II menu of configurations. Once a set of equivalent initial flaw sizes were calculated for each load definition for the smooth unnotched, open-hole and $K_{\mathrm{t}}=5$ specimens, then the mean and standard deviation values were determined at each combination of mean stress and maximum stress level. Multiple regression analyses were used to find the statistical relations for the mean as a function of the parameters under investigation, namely the geometric parameters and the loading related parameters. An identical approach was used to determine the standard deviation as a function of the same parameters. The statistical relations were developed for two assumed initial flaw types; one equation assuming that the crack started as a corner crack and another equation assuming that the crack started as a surface crack. These statistical models were verified by residual analyses and hypotheses testing.

From the established statistical relations the estimated mean and standard deviation of the distribution of equivalent initial flaw sizes were calculated for the $K_{\mathrm{t}}=3$ and $K_{\mathrm{t}}=4$ 
specimens. Using these estimated means and estimated standard deviations of the equivalent initial flaw sizes, random values of initial crack length were generated based on an assumed normal distribution. The randomly generated equivalent initial flaws sizes were used as the required input of initial crack size in the FASTRAN-II code to compute the estimated fatigue lives of each specimen for the load definition. The mean and variance of the estimated fatigue lives were computed and compared to the mean and variance of the experimentally measured fatigue lives using hypothesis testing. An acceptance statistical criterion for similarity of distributions was established, namely if either null hypotheses for both the mean and variance at a particular stress level were rejected at a level of significance, $\alpha$, of 0.05 then the distribution of the estimated fatigue lives were statistically different from the distribution of the measured fatigue lives. Alternatively if the null hypotheses associated with the mean and variance were both accepted at an $\alpha$ of 0.05 at a particular loading condition then the distribution of the estimated fatigue lives were identical to the distribution of measured fatigue lives at that loading condition. This methodology led to identical measured and computed distribution for some of the load conditions of the $K_{\mathrm{t}}=4$ specimens, but for only one of the load conditions associated with the $K_{\mathrm{t}}=3$ specimen. The initial crack configuration type did not have any significant effect on the proposed methodology.

Even though the proposed methodology was not able to estimate the fatigue lives for all fatigue load conditions and specimen types at an $\alpha$ of 0.05 , the methodology seems sound and the following recommendations should improve the capability of the methodology. The first suggestion would be to construct finite elements models of the flawed $K_{\mathrm{t}}=5$ specimen and determine the actual stress intensity factors and stress concentration factor associated with the as- 
built specimen. The next step would be to update all the necessary calculations using these new values.

The second suggestion involves the $K_{\mathrm{t}}=3$ specimen only. The experimental data used to validate results for the $K_{\mathrm{t}}=3$ specimen were collected from sample sizes that ranged between three and five specimens. This sample size is considered to be too small because it could introduce a significant amount of variability. Thus data collected from a larger sample size could reduce the variability in the test results and improve the comparison of the measured and computed fatigue lives. A sample size of eight to ten would be desirable.

Based on experimental observations it is recommended to update the statistical relations so that flaw sizes for open-hole specimens are based on corner flaw and for all other specimens on surface flaw only.

It was pointed out that measured crack growth history would allow back extrapolation curves to be superimposed on the crack growth history curves to assess the accuracy of the FASTRAN-II crack closure model. If necessary, fine tuning could then be introduced to create better agreement. Therefore, in future work, it is recommended that measured crack growth history data be collected.

The influence of stress gradient on equivalent initial flaw size must also be investigated. For open-hole and double-edge $\mathrm{V}$ notched specimens the presence of a hole and notch respectively causes stress gradient at symmetric plane of the specimens. The effect of the stress gradient on crack growth is significant and therefore must be considered in the statistical model. Finally, other statistical distributions, such as the Weibull or lognormal distribution, should be considered in addition to the normal distribution for the distribution of the assumed equivalent initial flaw sizes. 
REFERENCES 


\section{LIST OF REFERENCES}

[1] Newman, J. C., Jr., Phillips, E. P., and Swain, M. H., "Fatigue-Life Prediction Methodology Using Small-Crack Theory and a Crack-Closure Model," Proceedings of FAA-NASA Symposium on the Continued Airworthiness of Aircraft Structures DOT/FAA/AR-97/2, Vol. II, July 1997, pp. 331-356.

[2] Newman, J. C., Jr., "FASTRAN-II - A Fatigue Crack Growth Structural Analysis Program,” NASA TM-104159, Feb. 1992.

[3] Locke, J., "Data and Methodologies for Structural Life Evaluation of Small Airplanes," unpublished report of research conducted from 01/2004 to 2006.

[4] Gallagher, J. P., Giessler, F. J., Berens, A. P., and Engle, R. M., Jr., USAF Damage Tolerant Design Handbook: Guidelines for the Analysis and Design of Damage Tolerant Aircraft Structures, AFWAL-TR-82-3073, 1984.

[5] Manning, S. D., and Yang, J. N., USAF Durability Design Handbook: Guidelines for the Analysis and Design of Durable Aircraft Structures, AFWAL-TR-83-3027, 1984.

[6] Yang, J. N., and Manning, S. D., "Distribution of Equivalent Initial Flaw Size," , Proceedings of the Annual Reliability and Maintainability Symposium, San Francisco, CA, January 22-24, 1980, pp. 112-120.

[7] Schijve, J., "Significance of Fatigue Cracks in Micro-Range and Macro-Range," Fatigue Crack Propagation, ASTM STP 415, 1967, pp. 415-459.

[8] Newman, J. C., Jr., and Edwards, P. R., Short Crack Growth Behavior in an Aluminum Alloy: An AGARD Cooperative Test Programme, AGARD R-732, 1988.

[9] Newman, J. C., Jr., Phillips, E. P., Swain, M. H., and Everett, R. A., Jr., "Fatigue Mechanics - An Assessment of a Unified Approach to Life Prediction," Advances in Fatigue Lifetime Predictive Techniques, Proceeding of the Symposium, San Francisco, CA, $24^{\text {th }}$ April 1990, pp. 5-27.

[10] Newman, J. C., Jr., Wu, X. R., Venneri, S. L., and Li, C. G., "Small Crack Effects in High-Strength Aluminum Alloys," NASA-RP-1309, 1994.

[11] Pearson, S., "Initiation of Fatigue Cracks in Commercial Aluminum Alloys and the Subsequent Propagation of Very Short Cracks," Engineering Fracture Mechanics, Vol. 7, No. 2, 1975, pp. 235-247.

[12] Ritchie, R. O., and Lankford, J., "Small Fatigue Cracks," Proceedings of the Second International Conference/Workshop, Santa Barbara, CA, Jan. 5-10, 1986. 
[13] Miller, K. J., and de los Rios, E. R., The Behavior of Short Fatigue Cracks, Mechanical Engineering Publications, Suffolk, UK, 1986.

[14] Zocher, H., (ed.), Behaviour of Short Cracks in Airframe Components, AGARD CP-328, 1983.

[15] Edwards, P. R., and Newman, J. C., Jr., (eds.), Short-Crack Growth Behaviour in Various Aircraft Materials, AGARD Report No. 767, 1990.

[16] Elber, W., "The Significance of Fatigue Crack Closure," Damage Tolerance in Aircraft Structures, ASTM STP 486, 1971, pp. 230-242.

[17] Nisitani, H., and Takao, K. I., "Significance of Initiation, Propagation and Closure of Microcracks in High Cycle Fatigue of Ductile Materials," Engineering Fracture Mechanics, Vol. 15, No. 3, 1981, pp. 455-456.

[18] Minakawa, K., and McEvily, A. J., "On Near-Threshold Fatigue Crack Growth in Steels and Aluminum Alloys," Proceedings of the International Conference on Fatigue Thresholds, Vol. 2, 1981, pp. 373-390.

[19] Newman, J. C., Jr., "A Nonlinear Fracture Mechanics Approach to the Growth of Small Cracks," Behaviour of Short Cracks in Airframe Components, edited by H. Zocher, AGARD CP-328, 1983, pp. 6.1-6.26.

[20] Newman, J. C., Jr., "A Crack-Closure Model for Predicting Fatigue Crack Growth under Aircraft Spectrum Loading," Methods and Models for Predicting Fatigue Crack Growth under Random Loading, edited by J. Chang and C. Hudson, ASTM STP 748, 1981, pp. 53-84.

[21] Newman, J. C., Jr., "Fracture Mechanics Parameters for Small Fatigue Cracks," Small Crack Test Methods, ASTM STP 1149, edited by J. Allison and J. Larsen, 1992, pp. 6-28.

[22] Newman, J. C., Jr., "A Nonlinear Fracture Mechanics Approach to the Growth of Small Cracks," Behaviour of Short Cracks in Airframe Components, edited by H. Zocher, AGARD CP-328, 1983, pp. 6.1-6.26.

[23] Dugdale, D. S., "Yielding of Steel Sheets Containing Slits," Journal of Mechanics and Physics of Solids, Vol. 8, No. 2, 1960, pp. 100-104.

[24] Newman, J. C., Jr., Bigelow, C. A., and Shivakumar, K. N., "Three-Dimensional ElasticPlastic Finite-Element Analyses of Constraint Variations in Cracked Bodies," NASA TM 107704, January 1993.

[25] Yang, J. N., Manning, S. D., and Rudd, J. L., "Evaluation of a Stochastic Initial Fatigue Quality Model for Fastener Holes," Fatigue in Mechanically Fastened Composite and 
Metallic Joints, ASTM STP 927, edited by J. M. Potter, American Society for Testing and Materials, Philadelphia, 1986, pp. 118-149.

[26] Manning, S. D., Yang, J. N., and Rudd, J. L., "Durability of Aircraft Structures," Probabilistic Fracture Mechanics and Reliability, edited by J. W. Provan, Martinus Nijhoff Publishers, 1987, pp. 213-267.

[27] Manning, S. D., Speaker, S. M., Gordon, D. E., Yang, J. N., and Shinozuka, M., "Durability Methods Development, Volume 7 [Final Report, Jun. 1979 - Jan. 1984]," Air Force Flight Dynamics Laboratory, AFFDL-TR-79-3118-VOL-7, 1984.

[28] Yang, J. N., and Manning, S. D., "Demonstration of Probabilistic-Based Durability Analysis Method for Metallic Airframes," Journal of Aircraft, Vol. 27, No. 2, pp. 169175, February 1990.

[29] Yang, J. N., Manning, S. D., Rudd, J. L., and Bader, R. M., "Effect of Specimen Size and Notch Geometry on Equivalent Initial Flaw Size Distribution," AIAA/ASME/ASCE/AHS/ASC Structures, Structural Dynamics, and Materials Conference and Exhibit, 37 ${ }^{\text {th }}$. Salt Lake City, UT, April 15-17, 1996, Technical Papers Pt.1 (A9626801 06-39), Reston, VA, AIAA, 1996, pp. 385-397.

[30] Yang, J. N., Manning, S. D., and Newman, J. C., Jr., "Equivalent Initial Flaw Size Distribution for Notches in 2024-T3 Aluminum Accounting for Short Crack Effect," Proceedings of 1997 International Conference on Structural Safety and Reliability, Kyoto, Japan, November 24-28, 1997.

[31] Segerfrojd, G., Wang, G. S., Palmberg, B., and Blom, A. F., "Fatigue Behavior of Mechanical Joints: Critical Experiments and Statistical Analyses," Fatigue in New and Ageing Aircraft; Proceedings of the 19th ICAF Symposium, Edinburgh, United Kingdom, June 18-20, 1997. Vol. 1 (A98-29801 07-05), Engineering Materials Advisory Services, Ltd., 1997, pp. 575-598.

[32] Palmberg, B., "Equivalent Initial Flaw Sizes by Different Methods," Swedish Defence Research Agency, Stockholm, November, 2001.

[33] Newman, J. C., Jr., Wu, X. R., Swain, M. H., Zhao, W., Phillips, E. P., and Ding, C. F., "Small-Crack Growth and Fatigue Life Predictions for High-Strength Aluminium Alloys. Part II: Crack Closure and Fatigue Analyses," Fatigue and Fracture of Engineering Materials and Structures, Vol. 23, No. 1, Jan. 2000, pp. 59-72.

[34] Laz, P. J., and Hilberry, B. M., "Fatigue Life Prediction from Inclusion Initiated Cracks," International Journal of Fatigue, Vol. 20, No. 4, 1998, pp. 263-270.

[35] Gruenberg, K. M., Craig, B. A., and Hilberry, B. M., "Probabilistic Method for Predicting the Variability in Fatigue Behavior of 7075-T6 Aluminum," AIAA Journal, Vol. 37, No. 10, Oct. 1999, pp. 1304-1310. 
[36] Chan, S. K., Tuba, I. S., and Wilson, W. K., "On the Finite Element Method in Linear Fracture Mechanics," Engineering Fracture Mechanics, Vol. 2, 1970, pp. 1-17.

[37] Byskov, E., "The Calculation of Stress Intensity Factors Using the Finite Element Method with Cracked Elements," International Journal of Fracture, Vol. 6, 1970, pp. 159-167.

[38] Tracey, D. M., "Finite Elements for Determination of Crack Tip Elastic Stress Intensity Factors," Engineering Fracture Mechanics, Vol. 3, Oct. 1971, pp. 255-265.

[39] Tong, P., Pian, T. H. H., and Lasry, S. J., "A Hybrid-Element Approach to Crack Problems in Plane Elasticity," International Journal for Numerical Methods in Engineering, Vol. 7, No. 3, 1973, pp. 297-308.

[40] Papaioannou, S. G., Hilton, P. D., and Lucas, R. A., "A Finite Element Method for Calculating Stress Intensity Factors and Its Application to Composites," Engineering Fracture Mechanics, Vol. 6, Dec. 1974, pp. 807-823.

[41] Atluri, S. N., Kobayashi, A. S., and Nakagaki, M., "An Assumed Displacement Hybrid Finite Element Model for Linear Fracture Mechanics," International Journal of Fracture, Vol. 11, Apr. 1975, pp. 257-271.

[42] Benzley, S. F., "Representation of Singularities with Isoparametric Finite Elements," International Journal for Numerical Methods in Engineering, Vol. 8, No. 3, 1974, pp. 537-545.

[43] Ingraffea, A. R., and Wawrzynek, P. A., "Finite Element Methods for Linear Elastic Fracture Mechanics," Comprehensive Structural Integrity, de Borst, R. and Mang, H. A., (eds), Elsevier Science Ltd., Oxford, England, 2003. pp. 1-88.

[44] Henshell, R. D., and Shaw, K. G., "Crack Tip Finite Elements are Unnecessary," International Journal for Numerical Methods in Engineering, Vol. 9, No. 3, 1975, pp. 495-507.

[45] Barsoum, R. S., "On the Use of Isoparametric Finite Elements in Linear Fracture Mechanics," International Journal for Numerical Methods in Engineering, Vol. 10, No. 1, 1976, pp. 25-37.

[46] Barsoum, R. S., "Triangular Quarter-Point Elements as Elastic and Perfectly-Plastic Crack Tip Elements," International Journal for Numerical Methods in Engineering, Vol. 11, No. 1, 1977, pp. 85-98.

[47] Freese, C. E., and Tracey, D. M., "The Natural Isoparametric Triangle versus Collapsed Quadrilateral for Elastic Crack Analysis," International Journal of Fracture, Vol. 12, Oct. 1976, pp. 767-770. 
[48] Hussain, M. A., Coffin, L. F., and Zaleski, K. A., "Three-Dimensional Singular Elements," Computers and Structures, Vol. 13, 1981, pp. 595-599.

[49] Manu, C., "Quarter-Point Elements for Curved Crack Fronts," Computers and Structures, Vol. 17, No.2, 1983, pp. 227-231.

[50] Koers, R. W. J., "Use of Modified Standard 20-Node Isoparametric Brick Elements for Representing Stress/Strain Fields at a Crack Tip for Elastic and Perfectly Plastic Material," International Journal of Fracture, Vol. 40, 1989, pp. 79-110.

[51] MSC.Patran 2004, MSC Software Corporation, Santa Ana, CA, 2004.

[52] ZENCRACK, Version 7.3e, Zentech International Limited, Surrey, U.K., 2004.

[53] ABAQUS, Version 6.5, ABAQUS, Inc., Providence, RI, 2005.

[54] Newman, J. C., Jr., "A Crack-Opening Stress Equation for Fatigue Crack Growth," International Journal of Fracture, Vol. 24, 1984, R131-R135.

[55] Montgomery, D. C., Design and Analysis of Experiments, $5^{\text {nd }}$ ed., John Wiley and Sons, New York, 2001, Chaps. 10.

[56] Mendenhall, W., and Sincich, T., Statistics for Engineering and the Sciences, $4^{\text {th }}$ ed., Prentice Hall, New Jersey, 1995.

[57] STATGRAPHICS Plus, Version 5.0, StatPoint, Inc., Herndon, VA, 2000.

[58] Neter J., Wasseman, W., and Kunter, H. M., Applied Linear Statistical Models, $3^{\text {rd }}$ ed., Irwin, Homewood, IL, 1990.

[59] Samarah, I. K., "Response Surface Characterization Of Impact Damage And Residual Strength Degradation In Composites Sandwich Panels," Ph.D. Dissertation, Aerospace Engineering Department, Wichita State University, Wichita, KS, 2003.

[60] Myers R., and Montgomery, D. C., Response Surface Methodology, Wiley and Sons, New York, 1995. 
APPENDICES 


\section{APPENDIX A}

FASTRAN-II Input File for the Smooth Unnotched Specimen

A sample of the FASTRAN-II input file for the smooth unnotched specimen is given below. The detailed explanation of each input parameter can be found in Reference [2].

SC4: (SI UNITS) Mean Stress $=144.790 \mathrm{MPa}$, Maximum Stress $=210.290 \mathrm{MPa}$

cstamp 200.0

2024-T3

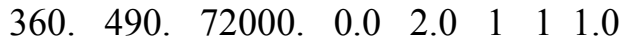

1

$\begin{array}{llllllll}5.0 \mathrm{E}-11 & 4.07 & 0.8 & 0.0 & 0.999 \mathrm{E}+04 & 2.0 & 267.0 & 1.0\end{array}$

$9 \quad 0$

$0.80 \quad 1.00 \mathrm{E}-12$

$1.10 \quad 5.00 \mathrm{E}-11$

$2.05 \quad 2.00 \mathrm{E}-09$

$4.00 \quad 8.00 \mathrm{E}-09$

$7.70 \quad 1.00 \mathrm{E}-07$

$13.50 \quad 1.00 \mathrm{E}-06$

$23.00 \quad 1.00 \mathrm{E}-05$

$36.00 \quad 1.00 \mathrm{E}-04$

$85.00 \quad 1.00 \mathrm{E}-02$

$\begin{array}{llllll}1.00 \mathrm{E}-07 & 2.00 & 1.0 & 2.50 \mathrm{E}-06 & 1.0 & 1.0\end{array}$ $\begin{array}{lllll}5000 & 5000 & 1 & 0 & 0.01\end{array}$

$\begin{array}{lllllll}99 & 0 & 0 & 4 & 0 & 0 & 0\end{array}$

Mechanical and fracture

properties

9.525e-3 4.6638014E-05 4.6638014E-05 4.6638014E-05 4.6638014E-05 4.6638014E-05 3.e-6 0.0 46

$\begin{array}{ll}0.0000 & 1.1220 \\ 0.0007 & 0.3921 \\ 0.0013 & 0.5288 \\ 0.0020 & 0.6176 \\ 0.0058 & 0.7983 \\ 0.0096 & 0.7849 \\ 0.0133 & 0.7392 \\ 0.0178 & 0.7212 \\ 0.0222 & 0.7272 \\ 0.0267 & 0.7381 \\ 0.0356 & 0.7458 \\ 0.0444 & 0.7429 \\ 0.0533 & 0.7413 \\ 0.0622 & 0.7477 \\ 0.0711 & 0.7578 \\ 0.0800 & 0.7670 \\ 0.0889 & 0.7726\end{array}$

Nondimensionalized crack length and boundary correction factor 


$\begin{array}{ll}0.0978 & 0.7769 \\ 0.1067 & 0.7830 \\ 0.1156 & 0.7928 \\ 0.1244 & 0.8058 \\ 0.1333 & 0.8209 \\ 0.1422 & 0.8374 \\ 0.1511 & 0.8547 \\ 0.1600 & 0.8722 \\ 0.1689 & 0.8903 \\ 0.1778 & 0.9114 \\ 0.1867 & 0.9385 \\ 0.2286 & 1.1598 \\ 0.2706 & 1.4611 \\ 0.3125 & 1.7631 \\ 0.3542 & 2.0164 \\ 0.3958 & 2.2258 \\ 0.4375 & 2.4016 \\ 0.4792 & 2.5827 \\ 0.5208 & 2.9198 \\ 0.5625 & 3.5697 \\ 0.6042 & 4.5632 \\ 0.6458 & 5.5284 \\ 0.6875 & 6.0430 \\ 0.7291 & 6.1974 \\ 0.7708 & 7.8766 \\ 0.8125 & 13.2091 \\ 0.8542 & 24.1186 \\ 0.8959 & 42.3604 \\ 0.9375 & 69.5488 \\ 9.525 \mathrm{e}-3 & \end{array}$

$9.525 \mathrm{e}-3$

$\begin{array}{ll}0.0 & 0.0\end{array}$

$\begin{array}{ll}0 & 0.0\end{array}$

$\begin{array}{llll}1 & 1 & 0 & 0\end{array}$

210.290

$\begin{array}{lll}1 & 1 & 1\end{array}$

$\begin{array}{lll}1.0 & 0.377 & 1000\end{array}$

$\begin{array}{lllll}0 & 0.0 & 0.0 & 0.0 & 0.0\end{array}$ HALT
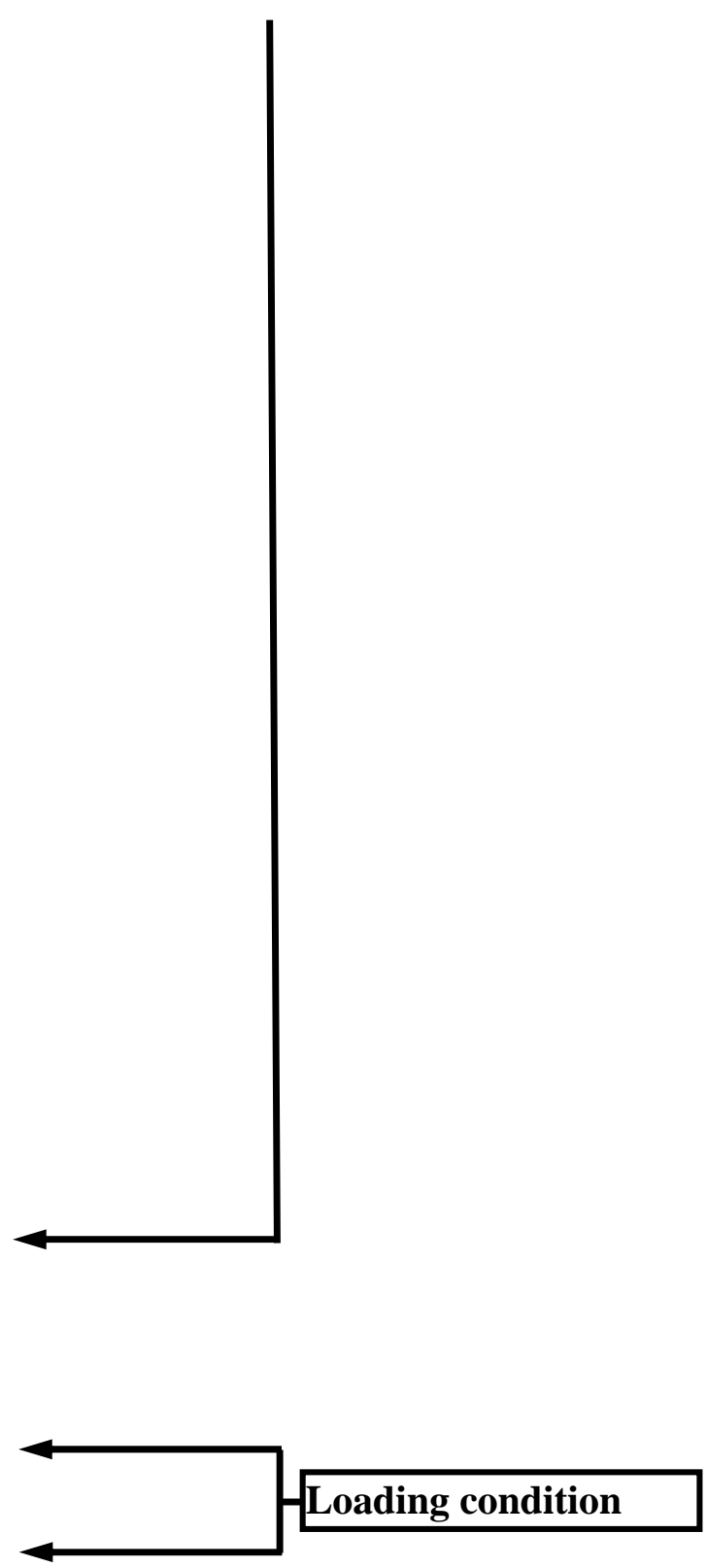


\section{APPENDIX B}

FORTRAN 90 Program to Determine the Equivalent Initial Flaw Size

A listing of the FORTRAN 90 program used for calculating equivalent initial flaw size for $K_{\mathrm{t}}=5$ specimen is given below.

program ifs_kt5_prog

use ifs $\mathrm{kt} 5 \mathrm{mod}$

implicit none

integer :: status

! This program calculates initial flaw size for $\mathrm{KT}=5$ specimen.

call ifs

end program ifs_kt5_prog

module ifs_kt5_mod

implicit none

integer,parameter :: q=selected_real_kind $(\mathrm{p}=12, \mathrm{r}=80)$

contains

! Calculates initial flaw size.

subroutine ifs

use dfport

implicit none

integer :: ioerr,status,id,life_ex,life_lo,life_up,life_c,f,errnum, \& count,flag

real $($ kind $=q)::$ ifs_lo,ifs_up,ifs_c,criteria1,criteria2,radr

character(len=110) $::$ line, add1, add2

character $(l e n=8):: \mathrm{idl}={ }^{\prime} 10.16 \mathrm{e}-3^{\prime}$

character $($ len $=10)::$ inputfile $=$ 'i.txt',outputfile $=$ 'o.txt'

life ex $=1127310$

life_lo $=1040367$

ifs_ $\bar{l} \mathrm{l}=1.3595 \mathrm{E}-05$

life_up $=1177616$ 
ifs_up $=1.3239 \mathrm{E}-05$

$\operatorname{read}\left(\mathrm{idl}, \mathrm{fmt}==^{\prime}(\mathrm{e} 8.2)^{\prime}\right) \mathrm{radr}$

count $=0$

flag $=0$

! Iterate.

iterate: do

if(flag.eq.0)then

! Secant method.

ifs_c=(ifs_up-ifs_lo) $*($ real(life_ex,q)-real(life_lo,q) $) /($ real(life_up,q) $-\&$

endif real(life_lo,q))+ifs_lo

flag $=0$

open(unit $=1$, file $=$ 's 1 i.txt',status $=$ 'old', action='read', iostat=status)

if(status.gt.0)then

write(unit=*,fmt='(3a)')"program could not open s1i.txt."

stop

endif

open(unit $=2$, file $=$ trim(inputfile), status $=$ 'replace', action=' ${ }^{\prime}$ rite',iostat=status)

if(status.gt.0)then

write(unit=*,fmt='(3a)')"program could not open ",trim(inputfile),"."

stop

endif

! Prepare input file with new initial flaw size value.

modify: do

read(unit $\left.=1, \mathrm{fmt}^{\prime}{ }^{\prime}(\mathrm{a})\right)^{\prime}$,iostat=ioerr)line

if(line.eq.")exit modify

if(ioerr.lt.0)exit modify

$\mathrm{id}=0$

id $=$ index $($ line, $i d l)$

if(id.ne.0)then

line $={ }^{\prime} \quad 53.34 \mathrm{e}-3^{\prime}$

write(add1,fmt $=$ '(es13.7)')ifs_c

write $\left(\operatorname{add} 2, \mathrm{fmt}={ }^{\prime}(\mathrm{es} 16.10)^{\prime}\right) \mathrm{ifs} \_\mathrm{c}+\mathrm{radr}$

line $=\operatorname{trim}($ line $) / / / / /$ add 1

line $=$ trim $($ line $) / / / / / \operatorname{add} 2$

line $=\operatorname{trim}($ line $) / / /^{\prime} \quad 1 / /$ add 1

line $=\operatorname{trim}($ line $) / / / / /$ add 2

line $=$ trim $($ line $) / / / / /$ add 1

line $=\operatorname{trim}($ line $) / /^{\prime} \quad ' / / /^{\prime} 3 . e-6 '$

line $=\operatorname{trim}($ line $) / /^{\prime} \quad 1 / /$ idl

endif 


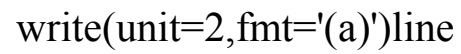

enddo modify

close (unit=1)

close (unit=2)

\section{! Execute FASTRAN-II.}

open (unit $=3$, file $=$ 'input.txt',status $=$ 'replace',action $=$ 'write',iostat $=$ status)

if(status.gt.0)then

write(unit=*,fmt='(a)')"program could not open input.txt."

stop

endif

write(unit $=3, \mathrm{fmt}==^{\prime}(\mathrm{a})$ ')trim(inputfile)

write(unit $=3, \mathrm{fmt}=$ '(a)')trim(outputfile)

$\mathrm{f}=$ system('fastran381 < input.txt')

if(f.eq. -1$)$ then

errnum=ierrno()

write(unit=*,fmt='(a,2x,i3)')"Error",errnum

endif

close(unit=3)

write(unit=*,fmt='(a,2x,es 13.7)')"ifs =",ifs_c

write(unit=*,fmt='(a,2x,i5)')" count $="$,count

! Read life value from output file.

open(unit $=4$,file $=$ trim(outputfile), status $=$ 'old', action $=$ 'read',iostat $=$ status)

if(status.gt.0)then

write(unit=*,fmt='(3a)')"program could not open ",trim(outputfile),"."

stop

endif

! Read output file.

inner: do read(unit $\left.=4, \mathrm{fmt}={ }^{\prime}(\mathrm{a})\right)^{\prime}$,iostat $=$ ioerr)line

if(ioerr.lt.0)exit inner

id $=0$

id=index(line,'LIMIT ON SIF SOLUTION FOR NTYP =-99 EXCEEDED')

if(id.ne.0)then

write(unit=*,fmt='(a)')" "

write(unit=*,fmt='(a)')" "

write $\left.(\text { unit }=* \text {,fmt }=(\mathrm{a}))^{\prime}\right) "$ PROBLEM "

write(unit $=*$,, $\left.\mathrm{mt}={ }^{\prime}(\mathrm{a}, 2 \mathrm{x}, \mathrm{es} 13.7)^{\prime}\right)$ "ifs $=$ ",ifs_c

ifs_c=ifs_c $+0.000001 \mathrm{e}-5$

write(unit $=*$,fmt='(a,2x,es13.7)')"ifs $="$,ifs_c

write(unit=*,fmt='(a)')" " 


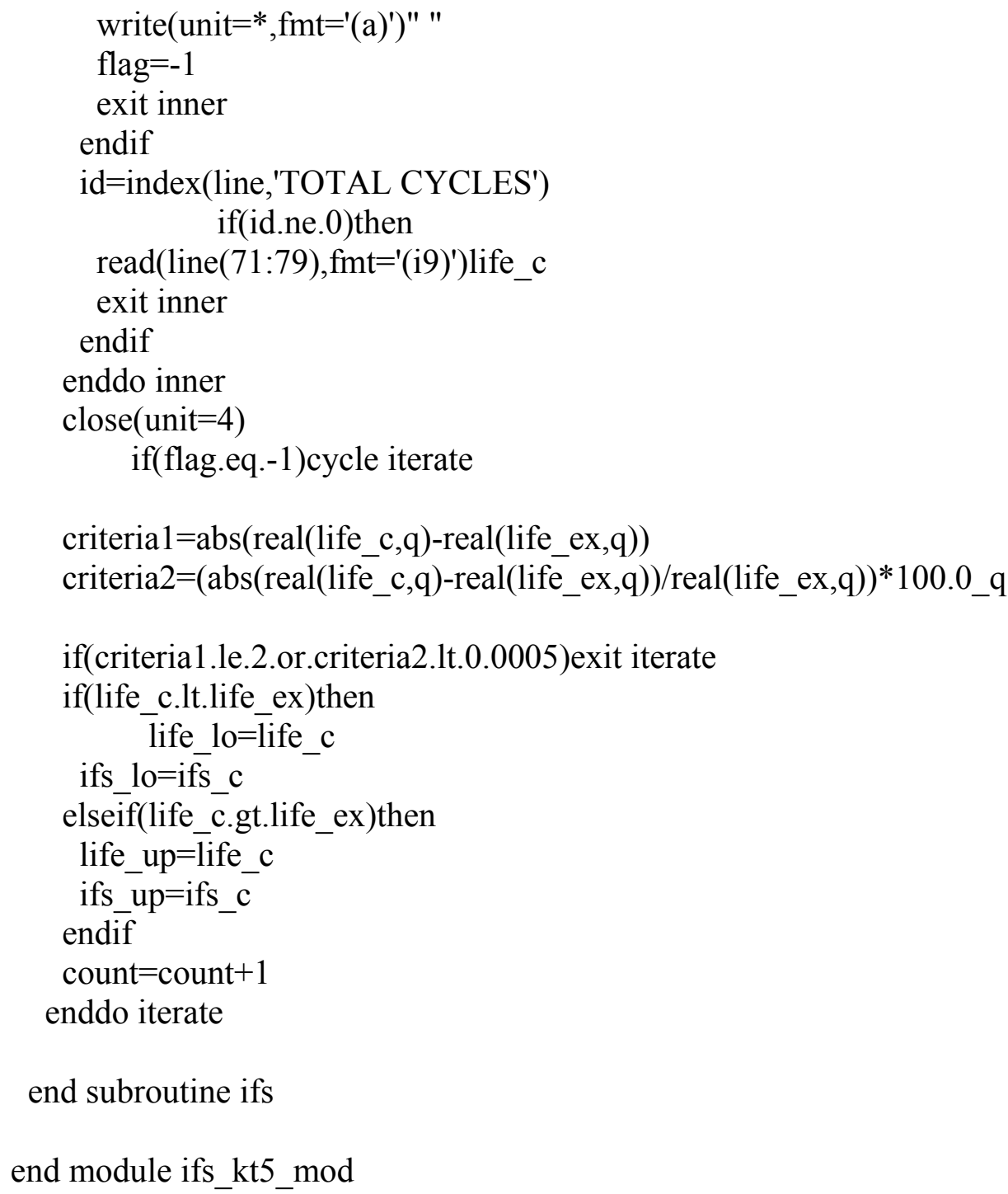




\section{APPENDIX C \\ Multiple Regression Analysis and Analysis of Variance Report}

This appendix contains the STATGRAPHICS Plus report of the multiple regression analyses and the analyses of variance for the equivalent mean and equivalent variance statistical relations. Both the equivalent mean and equivalent variance statistical relations were established for the assumed corner crack and surface crack configurations. Statistical relations were established for the following response variables: a) equivalent mean for assumed corner crack configuration b) equivalent mean for assumed surface configuration c) equivalent variance for assumed corner crack configuration and d) equivalent variance for assumed surface crack configuration. The statistical relation was established by regressing the response variable for the smooth unnotched specimen, the open-hole specimen, and the $K_{t}=5$ specimen against their respective geometric and loading conditions.

Tables C.1(a), C.2(a), C.3(a), and C.4(a) are the multiple regression analysis reports for equations (5.16) to (5.19) respectively and Tables C.1(b), C.2(b), C.3(b), and C.4(b) are the analysis of variance reports for equations (5.16) to (5.19) respectively. Column one of the multiple regression analysis report table contains the regressor variables, column two contains

the estimated coefficients, $\hat{\beta}_{i}$, while column three contains the test statistics, $t_{0}$, as defined in equation (5.44) and column four contains the $P$-values associated with $t_{0}$ as defined in equation (5.47) for each regressor variable. Column one of the analysis of variance report contains the sources of variation, column two contains the sum of squares values, column three contains the degrees of freedom associated with the each sum of squares, column four contains the mean regression sum of squares and mean error sum of squares, while column five contains the test 
statistic, $F_{0}$, as defined in equation (5.38) and column six contains the $P$-value associated with $F_{0}$ as defined in equation (5.41). The multiple coefficient of determination, $R^{2}$, and adjusted multiple coefficient of determination, $R_{\text {adj }}^{2}$ are also included in the analysis of variance report. 


\section{Table C.1(a)}

Multiple regression analysis report response variable: $\bar{c}_{0 \text {,corner }}^{\mathrm{eq}}$

\begin{tabular}{|c|c|c|c|}
\hline Parameter & $\begin{array}{c}\text { Regression } \\
\text { Coefficient }\end{array}$ & $t$-statistic & $P$-value \\
\hline CONSTANT & 0.000453704 & 0.312968 & 0.7581 \\
\hline$S_{\text {mean }}^{0.074} S_{\max }^{-1.056} K_{\mathrm{tg}}^{0.024}$ & 8.79502 & 4.48106 & 0.0003 \\
\hline$S_{\max }^{-0.981} w_{\mathrm{n}}^{0.045}$ & -5.31026 & -2.29246 & 0.0349 \\
\hline
\end{tabular}

Table C.1(b)

Analysis of variance report for response variable: $\bar{c}_{0, \text { corner }}^{\mathrm{eq}}$

\begin{tabular}{|c|c|c|c|c|c|}
\hline Source & $\begin{array}{c}\text { Sum of } \\
\text { Squares }\end{array}$ & $\begin{array}{c}\text { Degree of } \\
\text { Freedom }\end{array}$ & $\begin{array}{c}\text { Mean Sum } \\
\text { of Squares }\end{array}$ & $F$-ratio & $P$-value \\
\hline Regression & 0.0259185 & 2 & 0.0129592 & 1333.44 & 0.0000 \\
\hline Error & 0.000165216 & 17 & 0.0000097186 & - & - \\
\hline Total & 0.0260837 & 19 & - & - & - \\
\hline
\end{tabular}

Multiple Coefficient of Determination, $R^{2}=99.3666 \%$

Adjusted Multiple Coefficient of Determination, $R_{\text {adj }}^{2}=99.2921 \%$ 


\section{Table C.2(a)}

Multiple regression analysis report response variable: $s_{\text {corner }}^{\text {eq }}$

\begin{tabular}{|c|c|c|c|}
\hline Parameter & $\begin{array}{c}\text { Regression } \\
\text { Coefficient }\end{array}$ & $t$-statistic & $P$-value \\
\hline CONSTANT & -0.000668527 & -0.230783 & 0.8201 \\
\hline$S_{\operatorname{mean}}^{0.146} S_{\max }^{-1.239} K_{\mathrm{tg}}^{-0.108}$ & 10.2976 & 28.4878 & 0.0000 \\
\hline
\end{tabular}

Table C.2(b)

Analysis of variance report for response variable: $s_{\text {corner }}^{\mathrm{eq}}$

\begin{tabular}{|c|c|c|c|c|c|}
\hline Source & $\begin{array}{c}\text { Sum of } \\
\text { Squares }\end{array}$ & $\begin{array}{c}\text { Degree of } \\
\text { Freedom }\end{array}$ & $\begin{array}{c}\text { Mean Sum } \\
\text { of Squares }\end{array}$ & $F$-ratio & $P$-value \\
\hline Regression & 0.0357409 & 1 & 0.0357409 & 811.55 & 0.0000 \\
\hline Error & 0.00079272 & 18 & 0.00004404 & - & - \\
\hline Total & 0.0365336 & 19 & - & - & - \\
\hline
\end{tabular}

Multiple Coefficient of Determination, $R^{2}=97.8302 \%$

Adjusted Multiple Coefficient of Determination, $R_{\text {adj }}^{2}=97.7096 \%$ 


\section{Table C.3(a)}

Multiple regression analysis report response variable: $\bar{C}_{0, \text { surface }}^{\mathrm{eq}}$

\begin{tabular}{|c|c|c|c|}
\hline Parameter & $\begin{array}{c}\text { Regression } \\
\text { Coefficient }\end{array}$ & $t$-statistic & $P$-value \\
\hline CONSTANT & 0.000200679 & 0.191053 & 0.8507 \\
\hline$S_{\operatorname{man}}^{0.067} S_{\max }^{-1.035} K_{\mathrm{tg}}^{0.060}$ & 7.45709 & 4.8078 & 0.0002 \\
\hline$S_{\max }^{-0.975} w_{\mathrm{n}}^{0.093}$ & -5.49433 & -2.29508 & 0.0347 \\
\hline
\end{tabular}

Table C.3(b)

Analysis of variance report for response variable: $\bar{C}_{0, \text { surface }}^{\mathrm{eq}}$

\begin{tabular}{|c|c|c|c|c|c|}
\hline Source & $\begin{array}{c}\text { Sum of } \\
\text { Squares }\end{array}$ & $\begin{array}{c}\text { Degree of } \\
\text { Freedom }\end{array}$ & $\begin{array}{c}\text { Mean Sum } \\
\text { of Squares }\end{array}$ & $F$-ratio & $P$-value \\
\hline Regression & 0.02579585 & 2 & 0.0139792 & 2543.12 & 0.0000 \\
\hline Error & 0.000093447 & 17 & 0.0000054968 & - & - \\
\hline Total & 0.0280519 & 19 & - & - & - \\
\hline
\end{tabular}

Multiple Coefficient of Determination, $R^{2}=99.6669 \%$ Adjusted Multiple Coefficient of Determination, $R_{\text {adj }}^{2}=99.6277 \%$ 


\section{Table C.4(a)}

Multiple regression analysis report response variable: $S_{\text {surface }}^{\text {eq }}$

\begin{tabular}{|c|c|c|c|}
\hline Parameter & $\begin{array}{c}\text { Regression } \\
\text { Coefficient }\end{array}$ & $t$-statistic & $P$-value \\
\hline CONSTANT & -0.000352877 & -0.130086 & 0.8979 \\
\hline$S_{\operatorname{mean}}^{0.136} S_{\max }^{-1.210} K_{\mathrm{tg}}^{-0.050}$ & 8.80526 & 31.3208 & 0.0000 \\
\hline
\end{tabular}

Table C.4(b)

Analysis of variance report for response variable: $S_{\text {surface }}^{\text {eq }}$

\begin{tabular}{|c|c|c|c|c|c|}
\hline Source & $\begin{array}{c}\text { Sum of } \\
\text { Squares }\end{array}$ & $\begin{array}{c}\text { Degree of } \\
\text { Freedom }\end{array}$ & $\begin{array}{c}\text { Mean Sum } \\
\text { of Squares }\end{array}$ & $F$-ratio & $P$-value \\
\hline Regression & 0.0394203 & 1 & 0.0394203 & 980.99 & 0.0000 \\
\hline Error & 0.000723313 & 18 & 0.0000401841 & - & - \\
\hline Total & 0.0401436 & 19 & - & - & - \\
\hline
\end{tabular}

Multiple Coefficient of Determination, $R^{2}=98.1982 \%$

Adjusted Multiple Coefficient of Determination, $R_{\text {adj }}^{2}=98.0981 \%$ 


\section{APPENDIX D}

\section{Estimated Fatigue Lives}

This appendix contains the distribution of the estimated fatigue lives for the $K_{t}=3$ and $K_{\mathrm{t}}=4$ specimens. The distribution of estimated fatigue lives were computed using FASTRANII code using a distribution of random initial flaw sizes. The data of Tables D.1 and D.2 have been organized in the following manner. Tables D.1(a), D.1(b), and D.2(b) contain two major columns with each column containing two sub columns. Table D.2(a) contain three major columns with each column containing two sub columns. The major column contains values of the maximum stress, $S_{\max }$, while the sub columns contain the estimated fatigue lives for the corner and surface crack configuration respectively. 


\section{Table D.1(a)}

Estimated fatigue lives for $K_{\mathrm{t}}=3$ specimen and for $S_{\text {mean }}=20.684 \mathrm{MPa}$

\begin{tabular}{|c|c|c|c|}
\hline \multicolumn{2}{|c|}{$S_{\max }=68.948 \mathrm{~N} / \mathrm{mm}^{2}$} & \multicolumn{2}{|c|}{$S_{\max }=48.263 \mathrm{~N} / \mathrm{mm}^{2}$} \\
\hline$N_{\text {corner }}^{\mathrm{MC}}$ & $N_{\text {surface }}^{\mathrm{MC}}$ & $N_{\text {corner }}^{\mathrm{MC}}$ & $N_{\text {surface }}^{\mathrm{MC}}$ \\
\hline 43152 & 45950 & 189031 & 267408 \\
\hline 43338 & 46145 & 189222 & 263273 \\
\hline 44391 & 46539 & 189340 & 252601 \\
\hline 44427 & 47299 & 189888 & 252304 \\
\hline 44610 & 47980 & 191464 & 248815 \\
\hline 44739 & 49136 & 191650 & 247465 \\
\hline 44730 & 49216 & 191731 & 246222 \\
\hline 45624 & 49559 & 191865 & 245717 \\
\hline 45672 & 49666 & 192179 & 245345 \\
\hline 45926 & 49677 & 192379 & 244326 \\
\hline 46353 & 49730 & 192355 & 243469 \\
\hline 46387 & 49775 & 192709 & 242354 \\
\hline 46406 & 50121 & 193046 & 242194 \\
\hline 46481 & 50159 & 193047 & 241656 \\
\hline 46618 & 50272 & 193527 & 240723 \\
\hline 46845 & 50287 & 193741 & 240097 \\
\hline 46871 & 50362 & 193808 & 239053 \\
\hline 47011 & 50612 & 193963 & 238720 \\
\hline 47216 & 50717 & 194081 & 238546 \\
\hline 47215 & 51064 & 194213 & 238539 \\
\hline 47368 & 51352 & 194597 & 237985 \\
\hline 47381 & 51442 & 194837 & 237409 \\
\hline 47572 & 51482 & 194890 & 236733 \\
\hline 47576 & 51692 & 195096 & 236083 \\
\hline 47602 & 51699 & 195175 & 235401 \\
\hline 47641 & 51700 & 195242 & 233992 \\
\hline 47655 & 52011 & 195436 & 233371 \\
\hline 47664 & 52207 & 195639 & 233367 \\
\hline 47723 & 52257 & 195666 & 233081 \\
\hline 47816 & 52309 & 195716 & 233035 \\
\hline 48021 & 52651 & 196601 & 232310 \\
\hline 48115 & 52807 & 197054 & 231327 \\
\hline 48303 & 52846 & 197167 & 230827 \\
\hline 48351 & 52892 & 197177 & 230627 \\
\hline 48427 & 52975 & 197223 & 230611 \\
\hline 48615 & 53108 & 197823 & 230377 \\
\hline 48711 & 53134 & 198132 & 230141 \\
\hline
\end{tabular}




\begin{tabular}{|l|l|l|l|}
\hline 48827 & 53345 & 198166 & 230088 \\
\hline 48853 & 53377 & 198187 & 228340 \\
\hline 48869 & 53385 & 198595 & 228079 \\
\hline 48887 & 53402 & 198689 & 228040 \\
\hline 49300 & 53458 & 198750 & 227945 \\
\hline 49357 & 53805 & 199264 & 227653 \\
\hline 49559 & 53862 & 199442 & 227563 \\
\hline 49727 & 53929 & 199517 & 226668 \\
\hline 50158 & 54523 & 199593 & 225708 \\
\hline 50160 & 54549 & 199650 & 224653 \\
\hline 50291 & 54638 & 200186 & 224105 \\
\hline 50370 & 54769 & 200430 & 223648 \\
\hline 50392 & 54794 & 200603 & 223340 \\
\hline 50531 & 55119 & 200676 & 223018 \\
\hline 50673 & 55223 & 200813 & 222920 \\
\hline 51081 & 55290 & 201840 & 222867 \\
\hline 51213 & 55301 & 201922 & 222839 \\
\hline 51454 & 55334 & 202253 & 222407 \\
\hline 51574 & 55346 & 202274 & 222226 \\
\hline 51587 & 55625 & 202466 & 221767 \\
\hline 51614 & 55623 & 202644 & 221544 \\
\hline 51629 & 55905 & 202978 & 221339 \\
\hline 51867 & 56614 & 203720 & 220719 \\
\hline 52046 & 56673 & 203936 & 220318 \\
\hline 52154 & 56999 & 204348 & 220148 \\
\hline 52159 & 57378 & 204687 & 219994 \\
\hline 52366 & 57505 & 204815 & 218856 \\
\hline 52436 & 57596 & 206832 & 218638 \\
\hline 52598 & 57749 & 207071 & 218596 \\
\hline 52644 & 57775 & 207194 & 218176 \\
\hline 53253 & 57856 & 207442 & 217577 \\
\hline 53763 & 58211 & 207558 & 216208 \\
\hline 53842 & 58518 & 207961 & 215712 \\
\hline 53998 & 59163 & 210049 & 215343 \\
\hline 54018 & 59593 & 210394 & 214956 \\
\hline 54487 & 62435 & 218556 & 214751 \\
\hline 56697 & 62652 & 220592 & 211041 \\
\hline 58144 & 64248 & 223297 & 210564 \\
\hline
\end{tabular}




\section{Table D.1(b)}

Estimated fatigue lives for $K_{\mathrm{t}}=3$ specimen and for $S_{\text {mean }}=41.369 \mathrm{MPa}$

\begin{tabular}{|c|c|c|c|}
\hline \multicolumn{2}{|c|}{$S_{\max }=96.527 \mathrm{~N} / \mathrm{mm}^{2}$} & \multicolumn{2}{|c|}{$S_{\max }=62.053 \mathrm{~N} / \mathrm{mm}^{2}$} \\
\hline$N_{\text {corner }}^{\mathrm{MC}}$ & $N_{\text {surface }}^{\mathrm{MC}}$ & $N_{\text {corner }}^{\mathrm{MC}}$ & $N_{\text {surface }}^{\mathrm{MC}}$ \\
\hline 14384 & 18078 & 190070 & 272436 \\
\hline 14395 & 18344 & 207597 & 278159 \\
\hline 14643 & 18435 & 209048 & 279320 \\
\hline 14673 & 18572 & 225481 & 281304 \\
\hline 14710 & 18912 & 230782 & 283135 \\
\hline 14808 & 18979 & 235808 & 286223 \\
\hline 14878 & 19148 & 237782 & 289057 \\
\hline 15224 & 19241 & 242163 & 290822 \\
\hline 15337 & 19290 & 245415 & 294717 \\
\hline 15418 & 19550 & 248184 & 297609 \\
\hline 15468 & 19686 & 250430 & 298768 \\
\hline 15495 & 19882 & 253838 & 301935 \\
\hline 15518 & 19944 & 256397 & 302528 \\
\hline 15683 & 19981 & 257046 & 305938 \\
\hline 15685 & 20014 & 257697 & 313985 \\
\hline 15725 & 20101 & 258288 & 316921 \\
\hline 15760 & 20119 & 262591 & 319990 \\
\hline 15791 & 20228 & 262719 & 320070 \\
\hline 15907 & 20300 & 265977 & 321926 \\
\hline 15919 & 20523 & 266152 & 325574 \\
\hline 15967 & 20536 & 267302 & 325643 \\
\hline 16104 & 20637 & 267966 & 331404 \\
\hline 16106 & 20634 & 268601 & 335686 \\
\hline 16177 & 20666 & 268649 & 338262 \\
\hline 16226 & 20734 & 270643 & 342824 \\
\hline 16234 & 20791 & 272499 & 343833 \\
\hline 16260 & 20800 & 272766 & 346070 \\
\hline 16330 & 20960 & 273296 & 352518 \\
\hline 16457 & 21001 & 274582 & 353089 \\
\hline 16505 & 21155 & 277284 & 360878 \\
\hline 16619 & 21189 & 280423 & 362996 \\
\hline 16622 & 21214 & 281773 & 368915 \\
\hline 16661 & 21319 & 283227 & 381639 \\
\hline 16666 & 21348 & 286946 & 385803 \\
\hline 16701 & 21522 & 299517 & 389177 \\
\hline 16865 & 21532 & 300858 & 393502 \\
\hline 16920 & 21617 & 302098 & 393638 \\
\hline 16958 & 21832 & 302294 & 394626 \\
\hline
\end{tabular}




\begin{tabular}{|l|l|l|l|}
\hline 16983 & 21926 & 303377 & 395578 \\
\hline 17024 & 22026 & 307059 & 405445 \\
\hline 17171 & 22141 & 310461 & 414368 \\
\hline 17547 & 22254 & 316740 & 415981 \\
\hline 17707 & 22299 & 317748 & 418317 \\
\hline 17830 & 22433 & 326451 & 419139 \\
\hline 17940 & 22468 & 330761 & 423303 \\
\hline 18045 & 22493 & 345534 & 430204 \\
\hline 18057 & 22589 & 353989 & 439392 \\
\hline 18079 & 22691 & 355506 & 451775 \\
\hline 18168 & 23112 & 358967 & 453095 \\
\hline 18366 & 23158 & 380815 & 461526 \\
\hline 18644 & 23161 & 403602 & 462778 \\
\hline 18687 & 23278 & 422142 & 462981 \\
\hline 18813 & 23535 & 423789 & 474714 \\
\hline 18903 & 23686 & 426606 & 475758 \\
\hline 19076 & 23711 & 451385 & 510283 \\
\hline 19138 & 23805 & 490799 & 519937 \\
\hline 19231 & 24505 & 501627 & 520825 \\
\hline 19332 & 24519 & 506176 & 533490 \\
\hline 19693 & 24556 & 519710 & 559096 \\
\hline 20135 & 25093 & 538650 & 566084 \\
\hline 20649 & 25244 & 542108 & 572250 \\
\hline 20676 & 25334 & 542334 & 581525 \\
\hline 20872 & 25367 & 550541 & 611065 \\
\hline 20930 & 25905 & 550991 & 639343 \\
\hline 21014 & 25914 & 578938 & 643361 \\
\hline 21508 & 26682 & 661294 & 663478 \\
\hline 21594 & 26763 & 669370 & 674187 \\
\hline 21820 & 27429 & 670129 & 897879 \\
\hline 22005 & 28229 & 671962 & 900363 \\
\hline 22230 & 28785 & 708497 & 944376 \\
\hline 22425 & 29024 & 817614 & 1284267 \\
\hline 22616 & 29598 & 1284912 & 1295385 \\
\hline 24010 & 30334 & 1291055 & 1394651 \\
\hline 32561 & 31799 & 1362650 & 1441787 \\
\hline 38105 & 54395 & -- & 1984392 \\
\hline
\end{tabular}




\section{Table D.2(a)}

Estimated fatigue lives for $K_{\mathrm{t}}=4$ specimen and for $S_{\text {mean }}=20.684 \mathrm{MPa}$

\begin{tabular}{|c|c|c|c|c|c|}
\hline \multicolumn{2}{|c|}{$S_{\max }=62.053 \mathrm{~N} / \mathrm{mm}^{2}$} & \multicolumn{2}{|c|}{$S_{\max }=37.921 \mathrm{~N} / \mathrm{mm}^{2}$} & \multicolumn{2}{|c|}{$S_{\max }=32.061 \mathrm{~N} / \mathrm{mm}^{2}$} \\
\hline$N_{\text {corner }}^{\mathrm{MC}}$ & $N_{\text {surface }}^{\mathrm{MC}}$ & $N_{\text {corner }}^{\mathrm{MC}}$ & $N_{\text {surface }}^{\mathrm{MC}}$ & $N_{\text {corner }}^{\mathrm{MC}}$ & $N_{\text {surface }}^{\mathrm{MC}}$ \\
\hline 34308 & 35005 & 282187 & 314770 & 1126448 & 1934762 \\
\hline 36164 & 36465 & 284307 & 316710 & 1232732 & 2082059 \\
\hline 36184 & 38488 & 284601 & 318671 & 1325026 & 2294021 \\
\hline 36869 & 38624 & 289677 & 320098 & 1369634 & 2388405 \\
\hline 37206 & 39073 & 291557 & 320758 & 1541870 & 2389399 \\
\hline 37480 & 39189 & 291801 & 323022 & 1636764 & 2452668 \\
\hline 37589 & 39243 & 294602 & 324410 & 1696706 & 2532682 \\
\hline 37681 & 39383 & 299284 & 324682 & 1808235 & 2535413 \\
\hline 37847 & 39548 & 301407 & 326295 & 1847953 & 2554902 \\
\hline 38182 & 39619 & 302037 & 329011 & 1850461 & 2630845 \\
\hline 38348 & 39629 & 303087 & 330348 & 1857415 & 2643900 \\
\hline 38520 & 39779 & 303755 & 333623 & 1928047 & 2780870 \\
\hline 38742 & 40023 & 306367 & 335071 & 2072500 & 2810437 \\
\hline 39188 & 40150 & 307358 & 336271 & 2081003 & 2840581 \\
\hline 39232 & 40179 & 307501 & 336625 & 2132795 & 2967732 \\
\hline 39353 & 40577 & 308313 & 337170 & 2164212 & 3043174 \\
\hline 39416 & 40620 & 310700 & 339458 & 2209639 & 3054616 \\
\hline 39544 & 40865 & 311239 & 343464 & 2224372 & 3161278 \\
\hline 39763 & 40950 & 313525 & 347607 & 2282126 & 3161382 \\
\hline 39783 & 40982 & 314168 & 351232 & 2296076 & 3212780 \\
\hline 40004 & 40987 & 315684 & 353581 & 2308204 & 3354385 \\
\hline 40067 & 41236 & 317158 & 354132 & 2319673 & 3368834 \\
\hline 40148 & 41470 & 318602 & 355412 & 2410979 & 3388468 \\
\hline 40163 & 41779 & 318947 & 356771 & 2413283 & 3837201 \\
\hline 40372 & 41827 & 319492 & 359183 & 2468413 & 12190226 \\
\hline 40533 & 41876 & 319722 & 359545 & 2504140 & -- \\
\hline 40599 & 41909 & 320474 & 360072 & 2524927 & -- \\
\hline 40828 & 42067 & 320496 & 362408 & 2586228 & -- \\
\hline 40928 & 42301 & 321168 & 362541 & 2744867 & -- \\
\hline 41030 & 42333 & 321854 & 363118 & 2774589 & -- \\
\hline 41099 & 42525 & 321980 & 363665 & 2908222 & -- \\
\hline 41106 & 42583 & 322161 & 363978 & 2966852 & -- \\
\hline 41392 & 42757 & 323629 & 364252 & 3049680 & -- \\
\hline 41427 & 42826 & 325379 & 366339 & 3075724 & -- \\
\hline 41510 & 42881 & 326075 & 369459 & 3140613 & -- \\
\hline 41543 & 42895 & 329307 & 369995 & 3167849 & -- \\
\hline 41581 & 43013 & 330272 & 371895 & 3168391 & -- \\
\hline 41656 & 43082 & 331011 & 372653 & 3285096 & -- \\
\hline
\end{tabular}




\begin{tabular}{|c|c|c|c|c|c|}
\hline 41656 & 43088 & 331678 & 373341 & 3416637 & -- \\
\hline 41712 & 43253 & 333969 & 375516 & 3417895 & -- \\
\hline 41843 & 43299 & 334269 & 378084 & 3528719 & -- \\
\hline 41858 & 43337 & 334422 & 381878 & 3575547 & -- \\
\hline 41867 & 43410 & 335009 & 381887 & 3711406 & -- \\
\hline 41982 & 43675 & 336126 & 389397 & 3718729 & -- \\
\hline 42006 & 43698 & 337012 & 390602 & 3900106 & -- \\
\hline 42178 & 43719 & 338280 & 394886 & 8005763 & -- \\
\hline 42490 & 43864 & 340342 & 395175 & -- & -- \\
\hline 42619 & 43950 & 341332 & 396749 & -- & - \\
\hline 42718 & 44020 & 341737 & 400663 & -- & -- \\
\hline 42736 & 44025 & 343302 & 401012 & -- & -- \\
\hline 42949 & 44081 & 345670 & 411567 & -- & -- \\
\hline 43069 & 44254 & 349382 & 412068 & -- & -- \\
\hline 43137 & 44380 & 350329 & 414435 & -- & -- \\
\hline 43368 & 44396 & 351300 & 414604 & -- & -- \\
\hline 44051 & 44652 & 351541 & 427477 & -- & -- \\
\hline 44070 & 45323 & 352838 & 435992 & -- & -- \\
\hline 44247 & 45418 & 354958 & 437591 & -- & -- \\
\hline 44293 & 45686 & 355792 & 440876 & -- & -- \\
\hline 44304 & 45722 & 356153 & 443585 & -- & -- \\
\hline 44433 & 45825 & 357215 & 445829 & -- & -- \\
\hline 44655 & 45871 & 358004 & 447258 & -- & -- \\
\hline 44734 & 46052 & 360898 & 449673 & -- & -- \\
\hline 44757 & 46085 & 361205 & 465531 & -- & -- \\
\hline 45010 & 46224 & 363742 & 467472 & -- & -- \\
\hline 45099 & 46406 & 365127 & 484022 & -- & -- \\
\hline 45115 & 46523 & 365961 & 484882 & -- & -- \\
\hline 45464 & 46533 & 366877 & 485393 & -- & -- \\
\hline 46081 & 47113 & 383769 & 510056 & -- & -- \\
\hline 46105 & 47386 & 388296 & 517412 & -- & -- \\
\hline 46273 & 48165 & 396001 & 522049 & -- & -- \\
\hline 46462 & 48524 & 398811 & 653550 & -- & -- \\
\hline 48629 & 48867 & 450154 & 756590 & -- & - \\
\hline 49271 & 49077 & 520779 & 893306 & -- & -- \\
\hline 53505 & 50097 & 626227 & 1368472 & -- & -- \\
\hline 67532 & 67744 & 736758 & -- & -- & -- \\
\hline
\end{tabular}


Table D.2(b)

Estimated fatigue lives for $K_{\mathrm{t}}=4$ specimen and for $S_{\text {mean }}=41.368 \mathrm{MPa}$

\begin{tabular}{|c|c|c|c|}
\hline \multicolumn{2}{|c|}{$S_{\max }=75.842 \mathrm{~N} / \mathrm{mm}^{2}$} & \multicolumn{2}{|c|}{$S_{\max }=55.158 \mathrm{~N} / \mathrm{mm}^{2}$} \\
\hline$N_{\text {corner }}^{\mathrm{MC}}$ & $N_{\text {surface }}^{\mathrm{MC}}$ & $N_{\text {corner }}^{\mathrm{MC}}$ & $N_{\text {surface }}^{\mathrm{MC}}$ \\
\hline 25843 & 27100 & 366201 & 485109 \\
\hline 26086 & 27144 & 373667 & 754742 \\
\hline 26400 & 28658 & 395580 & 792117 \\
\hline 27103 & 28674 & 402791 & 822680 \\
\hline 27230 & 28720 & 404103 & 827986 \\
\hline 27263 & 29487 & 405586 & 907747 \\
\hline 27412 & 29580 & 405702 & 1149329 \\
\hline 27467 & 29693 & 424539 & 1167732 \\
\hline 27481 & 29755 & 440749 & 1177342 \\
\hline 27577 & 29849 & 449593 & 1188303 \\
\hline 27619 & 30031 & 456380 & 1268240 \\
\hline 27651 & 30137 & 487579 & 1272939 \\
\hline 27693 & 30211 & 499201 & 1287595 \\
\hline 28132 & 30254 & 500203 & 1371110 \\
\hline 28375 & 30267 & 507579 & 1451330 \\
\hline 28510 & 30482 & 516479 & 1468870 \\
\hline 28600 & 30611 & 517217 & 1523941 \\
\hline 28726 & 30724 & 565548 & 1567108 \\
\hline 28787 & 30791 & 594551 & 1752391 \\
\hline 29001 & 31045 & 599210 & -- \\
\hline 29009 & 31035 & 601243 & -- \\
\hline 29122 & 31093 & 618543 & -- \\
\hline 29395 & 31158 & 647484 & -- \\
\hline 29610 & 31219 & 662387 & -- \\
\hline 29760 & 31399 & 664561 & -- \\
\hline 29843 & 31484 & 669474 & -- \\
\hline 29850 & 31594 & 680577 & -- \\
\hline 29881 & 31608 & 703486 & -- \\
\hline 30088 & 31828 & 742487 & -- \\
\hline 30135 & 31973 & 831555 & -- \\
\hline 30193 & 32033 & 882207 & -- \\
\hline 30432 & 32152 & 1093072 & -- \\
\hline 30543 & 32430 & 1091606 & -- \\
\hline 30677 & 32716 & 1118391 & -- \\
\hline 31134 & 32734 & 1139520 & -- \\
\hline 31158 & 32757 & 1173236 & -- \\
\hline 31209 & 32780 & 1210225 & -- \\
\hline 31363 & 32830 & 1311205 & -- \\
\hline
\end{tabular}




\begin{tabular}{|c|c|c|c|}
\hline 31410 & 32926 & 1488472 & -- \\
\hline 31749 & 32928 & 1511242 & -- \\
\hline 31896 & 33054 & 1587577 & -- \\
\hline 31911 & 33207 & 1792387 & -- \\
\hline 32159 & 33222 & -- & -- \\
\hline 32161 & 33406 & -- & -- \\
\hline 32321 & 33447 & -- & -- \\
\hline 32328 & 33448 & -- & -- \\
\hline 32396 & 33478 & -- & -- \\
\hline 32507 & 33496 & -- & -- \\
\hline 32575 & 33549 & -- & -- \\
\hline 33362 & 33884 & -- & -- \\
\hline 33694 & 34045 & -- & -- \\
\hline 33746 & 34056 & -- & -- \\
\hline 33791 & 34072 & -- & -- \\
\hline 33950 & 34382 & -- & -- \\
\hline 34101 & 34406 & -- & -- \\
\hline 34545 & 34716 & -- & -- \\
\hline 34803 & 34741 & -- & -- \\
\hline 35010 & 35104 & -- & -- \\
\hline 35036 & 35527 & -- & -- \\
\hline 35551 & 36012 & -- & -- \\
\hline 35594 & 36289 & -- & -- \\
\hline 35946 & 37469 & -- & -- \\
\hline 37029 & 38046 & -- & -- \\
\hline 37654 & 38354 & -- & -- \\
\hline 38694 & 40665 & -- & -- \\
\hline 41029 & 41430 & -- & -- \\
\hline 41452 & 42449 & -- & -- \\
\hline 41998 & 42623 & -- & -- \\
\hline 42397 & 44386 & -- & -- \\
\hline 42817 & 44976 & -- & -- \\
\hline 42893 & 53359 & -- & -- \\
\hline 45133 & 56088 & -- & -- \\
\hline 53280 & 59972 & -- & -- \\
\hline 68794 & 71058 & -- & -- \\
\hline-- & 75678 & -- & -- \\
\hline
\end{tabular}

